RONNY CALIXTO CARBONARI

\title{
PROJETO DE ATUADORES PIEZELÉTRICOS FLEXTENSIONAIS USANDO O MÉTODO DE OTIMIZAÇÃO TOPOLÓGICA
}

Dissertação apresentada à Escola

Politécnica da Universidade de São Paulo para obtenção do título de Mestre em Engenharia. 
RONNY CALIXTO CARBONARI

\section{PROJETO DE ATUADORES PIEZELÉTRICOS FLEXTENSIONAIS USANDO O MÉTODO DE OTIMIZAÇÃO TOPOLÓGICA}

Dissertação apresentada à Escola Politécnica da Universidade de São Paulo para obtenção do título de Mestre em Engenharia.

Área de Concentração:

Engenharia Mecatrônica

Orientador:

Prof. Dr. Emílio Carlos Nelli Silva 
Este exemplar foi revisado e alterado em relação à versão original, sob responsabilidade única do autor e com a anuência de seu orientador.

São Paulo, 23 de abril de 2003.

Ronny Calixto Carbonari

Emílio Carlos Nelli Silva

\section{FICHA CATALOGRÁFICA}

Carbonari, Ronny Calixto

Projeto de Atuadores Piezelétricos Flextensionais Usando o Método de Otimização Topológica, São Paulo, 2003.

$168 \mathrm{p}$.

Edição Revisada - Elaborada pelo Serviço de Bibliotecas da EPUSP.

Dissertação (Mestrado) - Escola Politécnica da Universidade de São Paulo. Departamento de Engenharia Mecatrônica e de Sistemas Mecânicos.

1. Otimização Topológica 2. Atuadores Piezelétricos 3. Nanoposicionadores 4. MEMS.

I. Universidade de São Paulo. Escola Politécnica. Departamento 
Dedico este trabalho aos meus pais por me apoiarem e darem todas as condições de realizá-lo. 


\section{AGRADECIMENTOS}

Ao meu orientador, Prof. Dr. Emílio Carlos Nelli Silva, pela atenção, apoio, paciência, capacidade de orientação, liderança e pelo incentivo dado a mim e aos meus amigos a continuar o desenvolvimento de nossas carreiras acadêmicas.

Ao CNPq (Conselho Nacional de Desenvolvimento Científico e Tecnológico) pelo apoio financeiro deste trabalho, através de bolsa de mestrado.

Ao LNLS (Laboratório Nacional de Luz Síncrotron) pelo suporte dado pelo laboratório de microfabricação.

Aos funcionários do LNLS e em especial à Maria Helena e Ângelo Gobbi pela atenção e apoio dado na fabricação dos atuadores projetados neste trabalho.

Aos meus amigos Cícero Ribeiro de Lima, Gilder Nader, Paulo Godoy e Fernando de Freitas pela ajuda e incentivo.

À minha irmã pela ajuda e paciência nos momentos difíceis.

E por fim, a todos que direta ou indiretamente contribuíram para a realização deste trabalho. 


\section{RESUMO}

Atuadores Piezelétricos Flextensionais consistem de uma estrutura flexível atuada por cerâmicas piezelétricas (ou "pilhas" de cerâmicas). A estrutura flexível conectada a piezocerâmica deve gerar deslocamentos e forças em diferentes pontos específicos do domínio, para uma direção especificada. Estes atuadores são usados em aplicações de mecânica de precisão, tal como, sistemas microeletromecânicos (MEMS), manipulador de células, interferometria laser, equipamentos de nanotecnologia, equipamentos de microcirurgias, nanoposicionadores, sonda de varredura microscópica, e etc. Porém, devido ao fato destes atuadores consistirem principalmente de um mecanismo flexível, seu projeto é complexo. A estrutura flexível comporta-se como um transformador mecânico pela amplificação para converter, direcionar e amplificar os pequenos deslocamentos gerados pela piezocerâmica (ordem de nanômetros). A estrutura flexível é projetada distribuindose flexibilidade e rigidez no domínio de projeto, o que pode ser obtido usando a otimização topológica. Portanto, o objetivo deste trabalho é implementar um método sistemático baseado no método de otimização topológica para projetar atuadores piezelétricos flextensionais. Essencialmente, o método de otimização topológica consiste em encontrar a distribuição ótima de material perfurando o domínio de projeto com infinitos microfuros. $\mathrm{O}$ material em cada ponto pode alterar de vazio a total presença de material, também assumindo material intermediário (ou compósito). A implementação do método de otimização topológica é baseado no modelo de material SIMP (Simple Isotropic Material with Penalization). O problema de otimização é posto como a maximização dos deslocamentos gerados (ou força de blocagem) em diferentes pontos e direções especificadas do domínio. Considerando o comportamento linear da piezocerâmica.

Alterando a flexibilidade e a rigidez da estrutura flexível conectada a piezocerâmica obtém-se diferentes tipos de atuadores piezelétricos flextensionais, que podem ser projetados para determinadas aplicações. Para ilustrar o método, os exemplos mostrados são modelos bidimensionais (2D), uma vez que a maior parte 
das aplicações envolve dispositivos planos. Estes atuadores são fabricados usando corrosão química em chapas de cobre abaixo de $200 \mu \mathrm{m}$ de espessura através do método de litografia. Técnica de corrosão química tem um baixo custo e permite-nos fabricar diversos protótipos para testes. Esta técnica pode ser facilmente utilizada no LNLS (Laboratório Nacional de Luz Síncrotron - Campinas). Análise experimental destes protótipos são procedidas para medição de deslocamentos usando uma Probe Station. Como trabalho futuro, estes protótipos serão construídos em escala de MEMS. 


\section{ABSTRACT}

Flextensional Piezoelectric Actuators consist of a flexible structure actuated by piezoelectric ceramics (or a stack of piezoceramics). The flexible structure connected to the piezoceramic must generate displacements and forces in different specified points of the domain, according to a specific direction. These actuators are applied to precision mechanic such as microelectromechanical systems (MEMS), cell manipulators, laser interferometers, nanotechnology equipment, microsurgery equipment, nanopositioners, scanning probe microscopy, etc. However, due to the fact these actuators essentially consist of a compliant mechanism their design is complex. The compliant structure behaves as a mechanical transform by amplifying and changing the direction of small output displacements generated by piezoceramics (order of nanometer). The flexible structure is designed by distributing flexibility and stiffness in the design domain, which can be archieved by using topology optimization. Therefore, the objective of this work is to implement a systematic method based on topology optimization method to design flextensional piezoelectric actuators. Essentially, the topology optimization method consists of finding the optimal material distribution in a perforated design domain with infinite microvoids. The material in each point can change from void to full material, also assuming intermediate (or composite) material. The implemented topology optimization method is based on the SIMP (Simple Isotropic Material with Penalization) material model. The optimization problem is posed as maximization of output displacements (or grabbing forces) in different specified directions and points of the domain. A linear behavior of piezoceramic is considered.

By changing the flexibility and stiffness of flexible structure connected to the piezoceramics different types of flextensional piezoelectric actuators can be designed for a desired application. To illustrate the method, examples presented herein are limited to two-dimensional (2D) models once in most part of applications of these actuators they are planar devices. These actuators are manufactured by using chemical corrosion on a 200 um thickness copper plate through lithography method. 
Chemical corrosion technique has a low cost and it allow us to manufacture several prototypes for testing. For this technique, facilities of the micromachining laboratory of National Sincroton Light Laboratory (LNLS - Campinas) are used. Experimental analysis of these prototypes are conducted by measuring displacements using a probe station. As a future work, these prototypes will be built in a MEMS scale. 


\section{SUMÁRIO}

LISTA DE FIGURAS

LISTA DE TABELAS

LISTA DE ABREVIATURAS E SIGLAS

LISTA DE SÍMBOLOS

CAPÍTUlO 1: Atuadores Piezelétricos Flextensionais ......................................... 1

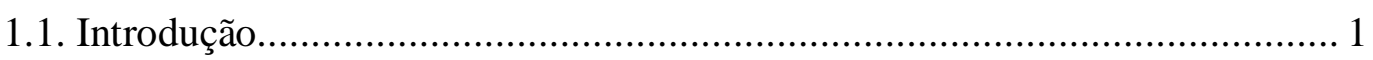

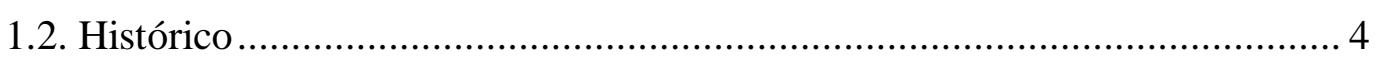

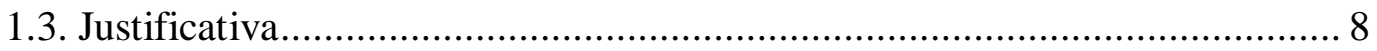

CAPÍTULO 2: Otimização Topológica............................................................... 10

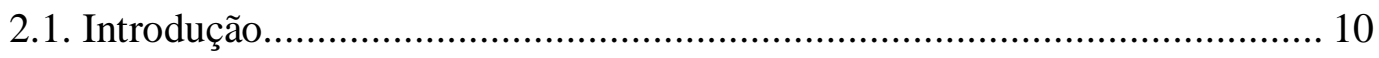

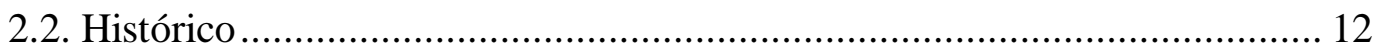

2.3. Procedimentos para implementação da OT............................................. 15

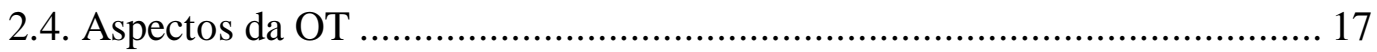

2.4.1. OT de estruturas de malhas contínuas ......................................... 17

2.4.2. OT de estruturas discretas.......................................................... 18

2.4.3. OT de um meio contínuo .............................................................. 19

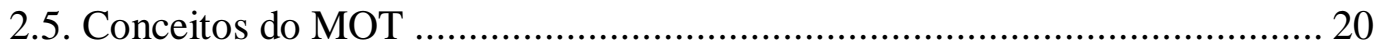

2.5.1. Domínio estendido fixo de projeto............................................... 21

2.5.2. Modelo de material................................................................... 22

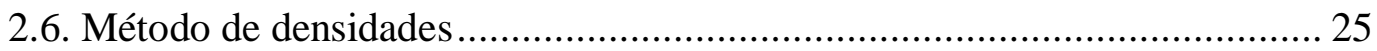

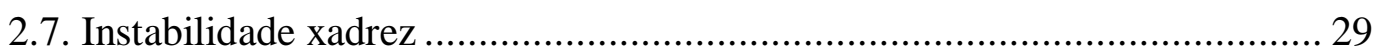

CAPÍTULO 3: Piezeletricidade e o MEF piezelétrico....................................... 34

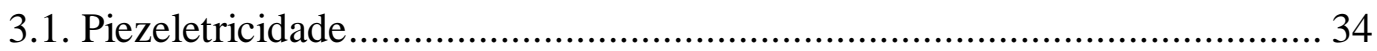


3.2. Material Piezelétrico

3.3. Equações Constitutivas Piezelétricas na forma Tensorial e Matricial............36

3.3.1. Estado Plano de Tensões Mecânicas ................................................ 44

3.3.2. Estado Plano de Deformações Mecânicas ..................................... 45

3.4. Implementação do MEF piezelétrico ...................................................... 47

3.4.1. Implementação do Elemento Isoparamétrico de quatro nós............. 49

3.4.2. Formulação da Matriz do Elemento .............................................. 52

3.5. Determinação dos deslocamentos Nodais ................................................. 56

\section{CAPÍTUlO 4: Formulação do Problema de OT para Projetos de Atuadores} Piezelétricos Flextensionais..................................................................................... 58

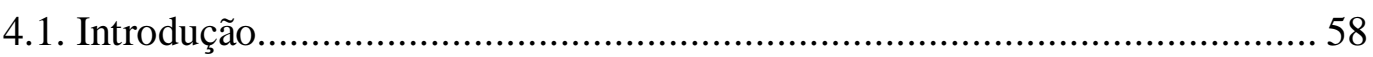

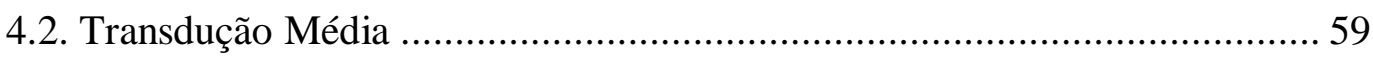

4.3. Descrição do Problema de Otimização para o Projeto de Atuadores

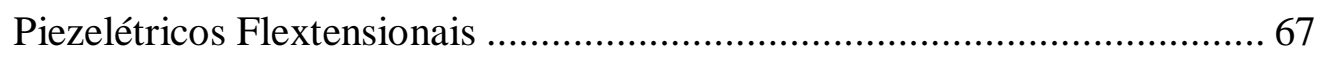

4.3.1. Formulação do Problema para Atender à função Eletromecânica.... 67

4.3.2. Formulação do Problema para Atender à função Estrutural............. 70

4.4. Função Multi-objetivo e formulação do problema. ................................... 72

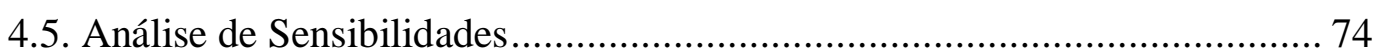

CAPÍTULO 5: Implementação Numérica ............................................................ 79

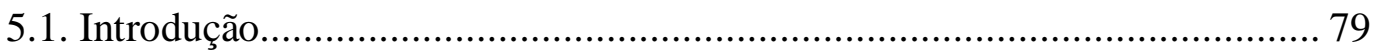

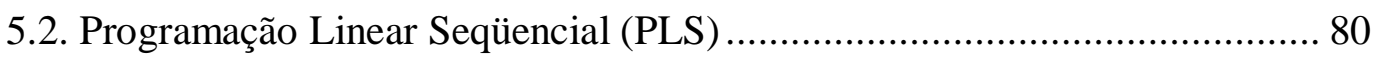

5.3. Filtro para eliminação da "instabilidade xadrez" ..................................... 87

5.4. Procedimento para Implementação do MOT ............................................. 90

CAPÍTULO 6: Resultados...............................................................................94

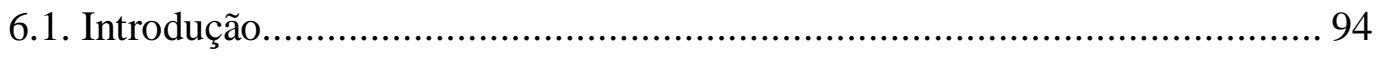

6.2. Influência dos parâmetros do MOT no projeto do Atuador Piezelétrico...... 96

6.2.1. Exemplo 1 - variação ao valor inicial de densidades...................... 97

6.2.2. Exemplo 2 - variação da restrição de volume .............................. 100

6.2.3. Exemplo 3 - variação do coeficiente $w$........................................ 104 
6.2.4. Exemplo4 - variação na discretização do domínio inicial 108

6.3. Formação e Eliminação da "instabilidade xadrez" .................................. 112

6.3.1. Solução para tratar modelos com alta discretização..........................112

6.3.2. Exemplos - de eliminação da "instabilidade xadrez"........................114

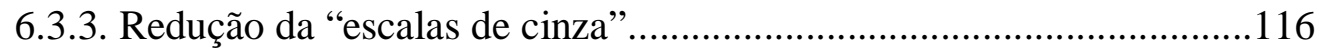

6.4. Verificação dos resultados obtidos ........................................................ 123

6.5. Projeto de atuadores piezelétricos flextensionais .................................... 126

6.5.1. Processo de fabricação ............................................................. 128

6.5.2. Exemplos de atuadores piezelétricos flextensionais projetados ....... 129

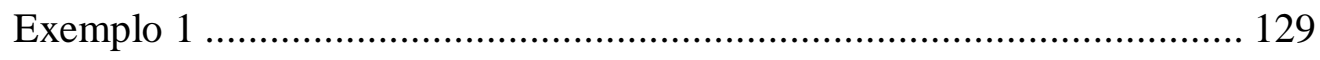

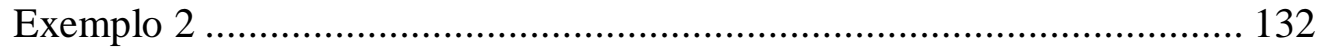

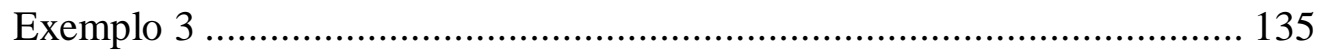

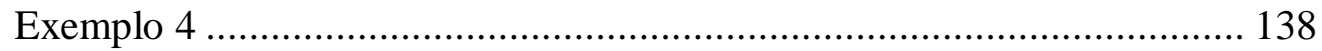

6.6. Verificações dos resultados obtidos no Exemplo 3 ................................. 141

CAPÍtUlO 7: Conclusão............................................................................... 146

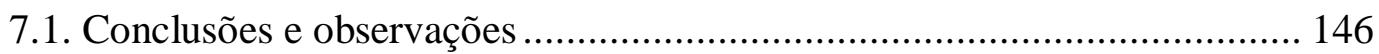

7.2. Considerações finais e trabalhos futuros............................................. 147

ANEXO A: Descrição da utilização do problema de otimização para projeto de atuadores piezelétricos flextensionais ............................................................ 148

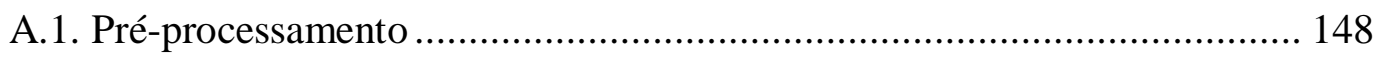

A.2. Processamento com o APFLEX …...................................................... 149

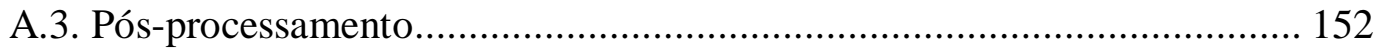

REFERÊNCIAS BIBLIOGRÁFICAS .......................................................... 154 


\section{LISTA DE FIGURAS}

Figura 1.1 - Atuador Piezelétrico Flextensional...................................................... 1

Figura 1.2 - (a) Atuador "moonie"; (b) Atuador "moonie entalhado"; (c) Atuador

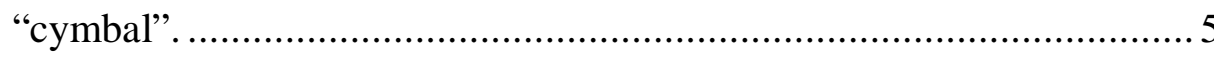

Figura 2.1 - Exemplo de 3 categorias de otimização estrutural: (a) otimização paramétrica; (c) otimização de forma e (e) otimização topológica....... 11

Figura 2.2 - Procedimento típico de projeto estrutural por otimização topológica... 16

Figura 2.3 - Otimização estrutural obtida por Michell. ........................................ 18

Figura 2.4 - Otimização topológica de uma estrutura discreta.............................. 19

Figura 2.5 - Resultado da Otimização Topológica do problema resolvido anteriormente por Michell. 20

Figura 2.6 - Conceitos de domínios para otimização de forma (a) e topológica (b). 21

Figura 2.7 - Definição do domínio de projeto e das condições de contorno. 22

Figura 2.8 - Microestrutura para o método da homogeneização. 24

Figura 2.9 - Curva limite de Hashin-Shtrikman e curvas do modelo SIMP............. 28

Figura 2.10 - Instabilidade xadrez. 29

Figura 2.11 - Arranjos da "instabilidade xadrez" e de material distribuído uniformemente. 30

Figura 3.1 -Cristalografia do cristal PZT: (a) não-polarizado e (b) polarizado. 35

Figura 3.2 - Diagrama entre as iterações mecânicas e elétricas. 36 
Figura 3.3 - (a) estado plano de tensões mecânicas (EPTM); (b) estado plano de deformações mecânicas (EPDM). A polarização $(\mathrm{P})$ de um material é considerada na direção 3

Figura 3.4 - Apresentação esquemática do MEF.

Figura 3.5 - Mapas de coordenadas isoparamétricas. (a) elemento em coordenadas locais, (b) elemento em coordenadas globais.

Figura 4.1 - Teorema da reciprocidade na elasticidade. 60

Figura 4.2 - Uma extensão do teorema da reciprocidade para a piezeletricidade..... 63

Figura 4.3 - Casos de carga para cálculo da transdução média (caso a) e flexibilidade média (b). 68

Figura 5.1 - Limites móveis. 83

Figura 5.2 - Raio de abrangência nos filtros espaciais. 88

Figura 5.3 - Fluxo do processo iterativo de otimização 91

Figura 5.4 - Situações de carregamento para o projeto do atuador piezelétrico. 92

Figura 6.1 - Domínio inicial de projeto do atuador. 97

Figura 6.2 - Topologia ótima para diferentes valores de denidades iniciais. 99

Figura 6.3 - Gráficos de convergência..... 100

Figura 6.4 - Domínio inicial de projeto do atuador. 101

Figura 6.5 - Efeito da variação da restrição de volume no projeto do atuador piezelétrico flextensional. 102

Figura 6.6 - Ponto principal de movimento da estrutura flexível da Fig. 6.5. 103

Figura 6.7 - Gráficos de convergência $\left(V^{*}=20 \%\right)$. 104

Figura 6.8 - Domínio inicial de projeto do atuador. 105

Figura 6.9 - Efeito da alteração do coeficiente $w$ na OT do atuador. 106 


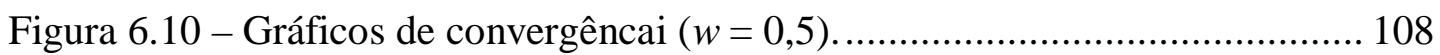

Figura 6.11 - Domínio inicial de projeto do atuador............................................. 109

Figura 6.12 - Configurações de topologia ótima para diferentes malhas. .............. 110

Figura 6.13 - Gráficos de convergência (malha com 818 elementos)................... 111

Figura 6.14 - Configuração de topologia ótima para o exemplo do item 6.2.1, considerando o valor inicial de densidades igual à 0,15 e uma malha de 2500 elementos.

Figura 6.15 - Configuração de topologia ótima para o exemplo do item 6.2.2 considerando $V^{*}=20 \%$ e uma malha de 2500 elementos.

Figura 6.16 - Configuração de topologia ótima para o exemplo do item 6.2.3, considerando $w=0,80, V^{*}=15 \%$ e uma malha de 2500 elementos .. 113

Figura 6.17 - Configuração de topologia ótima para o exemplo do item 6.2.4, considerando uma malha de 2556 elementos.

Figura 6.18 - Topologia ótima obtida com a utilização do filtro para o domínio inicial do item 6.2.1

Figura 6.19 - Topologia ótima obtida com a utilização do filtro para o domínio inicial do item 6.2.2

Figura 6.20 - Topologia ótima obtida com a utilização do filtro para o domínio inicial do item 6.2.3.

Figura 6.21 - Topologia ótima obtida com a utilização do filtro para o domínio inicial do item 6.2.4.

Figura 6.22 - Configuração final da topologia para o domínio inicial do item 6.2.1.

Figura 6.23 - Configuração final da topologia para o domínio inicial do item 6.2.2.

Figura 6.24 - Configuração final da topologia para o domínio inicial do item 6.2.3. 
Figura 6.25 - Configuração final da topologia para o domínio inicial do item 6.2.4.

Figura 6.26 - Domínio inicial de projeto do atuador.

Figura 6.27 - Topologia ótima obtida SEM a utilização do filtro espacial.

Figura 6.28 - Topologia ótima obtida COM a utilização do filtro espacial.

Figura 6.29 - Configuração final da topologia ótima obtida com o desligamento do filtro nas últimas iterções

Figura 6.30 -Efeito da alteração do tamanho do raio de abrangência do filtro nos resultados.

Figura 6.31 - Resultado da topologia ótima obtida por Silva; Nishiwaki; Kikuchi (2000), considerando condições semelhantes do item 6.2.1.

Figura 6.32 - Resultado da topologia ótima obtida Silva; Nishiwaki; Kikuchi (2000), considerando condições semelhantes do item 6.2.3.

Figura 6.33 - Resultado da topologia ótima da Fig. 6.16 sem as condições de simetria.

Figura 6.34 - Resultado da topologia ótima obtida e deformada (SILVA;

NISHIWAKI; KIKUCHI, 1999), considerando condições semelhantes do item 6.2.4. 125

Figura 6.35 - Deformada da topologia ótima da Fig. 6.17. 126

Figura 6.36 - Domínio inicial do atuador. 130

Figura 6.37 - Configuração da topologia ótima. 130

Figura 6.38 -Deformada da topologia ótima. 131

Figura 6.39 - Distribuição de tensões mecânicas (Von-Mises), unidades em $P a \ldots 131$

Figura 6.40 - Protótipo do atuador (escala, 1 divisão =0,1 $\mathrm{mm}$ ). 132

Figura 6.41 - Domínio inicial do atuador. 133

Figura 6.42 - Configuração da topologia ótima. 133

Figura 6.43 -Deformada da topologia ótima. 
Figura 6.44 - Distribuição de tensões mecânicas (Von-Mises), unidades em Pa. .. 134

Figura 6.45 - Protótipo do atuador (escala, 1 divisão = 1,0mm)..

Figura 6.46 - Domínio inicial do atuador

Figura 6.47 - Configuração da topologia ótima.

Figura 6.48 -Deformada da topologia ótima.

Figura 6.49 - Distribuição de tensões mecânicas (Von-Mises), unidades em $P a$... 137

Figura 6.50 - Protótipo do atuador (escala, 1 divisão $=1,0 \mathrm{~mm}$ ) 138

Figura 6.51 - Domínio inicial do atuador. 139

Figura 6.52 - Configuração da topologia ótima.

Figura 6.53 -Deformada da topologia ótima. 140

Figura 6.54 - Distribuição de tensões mecânicas (Von-Mises), unidades em $P a \ldots 140$

Figura 6.55 - Protótipo do atuador (escala, 1 divisão $=1,0 \mathrm{~mm}$ ) 141

Figura 6.56 - Deformada do modelo 3D. 142

Figura 6.57 - Distribuição de tensões mecânicas (Von-Mises), unidades em $P a$... 143

Figura 6.58 - Distribuição de tensões mecânicas (Von-Mises), unidades em $P a$... 144 Figura 6.59 - Deformada do modelo 3D A. 145

Figura A.1 - Fluxo básico da estrutura de utilização do APFLEX. 148

Figura A.2 - Fluxo geral de funcionamento do APFLEX. 150

Figura A.3 - Fluxo da rotina de MEF. 152 


\section{LISTA DE TABELAS}

Tabela 6.1 - Propriedade dos Materiais.

Figura 6.2 - Valores da Transdução média e Flexibilidade média para diferentes valores de $V^{*}$ para os atuadores deste exemplo.....

Tabela 6.3 - Valores da Transdução média e Flexibilidade média para diferentes valores de $w$ para os atuadores deste exemplo

Tabela 6.4 - Valores dos deslocamentos para o ponto de atuação. 141

Tabela 6.5 - Valores de deslocamentos para o ponto de atuação 145 


\section{LISTA DE ABREVIATURAS E SIGLAS}

\begin{tabular}{ll} 
2D & Bidimensional \\
3D & Tridimensional \\
ANSYS & Software Comercial de Elementos Finitos \\
ASCII & American Standard Code for Information Interchange \\
CAD & Computer Aided Design \\
DSPLP & Rotina de programação linear que usa o método de Kamarkar \\
EUA & Estados Unidos da América \\
MEF & Método dos Elementos Finitos \\
MEMS & Microelectromechanical Systems \\
MOT & Método de Otimização Topológica \\
APFLEX & Software de Otimização Topológica desenvolvido neste trabalho \\
OT & Otimização Topológica \\
PL & Programação Linear \\
PLS & Programação Linear Seqüencial \\
PQS & Programação Quadrática Seqüencial \\
SAND & Simultaneous Analysis and Design \\
SIMP & Simple Isotropic Material with Penalization \\
SIMPLX & Rotina de Programação Linear que usa o método Simplex \\
Eq. & Equação \\
Fig. & Figura \\
Tab. & Tabela \\
GPa & Giga pascal \\
mm & milímetros \\
obj & Joule \\
\hline micro Coulumb \\
\hline Arquivo ponto objeto
\end{tabular}




\section{LISTA DE SÍMBOLOS}

\begin{tabular}{|c|c|}
\hline$\xi$ & Abscissa do sistema de coordenadas locais do elemento \\
\hline$w$ & Coeficiente de atribuição de peso na função objetivo \\
\hline$\Gamma_{t^{k}}$ & Contorno com carregamento mecânico aplicado \\
\hline$\Gamma_{d^{k}}$ & Contorno com carregamento elétrico aplicado \\
\hline$\Gamma_{\mathrm{u}}$ & Contorno restringido mecanicamente \\
\hline$\Gamma_{\phi}$ & Contorno restringido eletricamente \\
\hline$\rho_{\mathrm{e}}$ & Densidade do elemento \\
\hline$R_{i j}$ & Distância entre centróides do elemento central $i$ e do elemento vizinho \\
\hline$\Omega$ & Domínio estendido fixo de projeto \\
\hline$\Pi$ & Energia total \\
\hline$e(x)$ & Erro da linearização da função através da Série de Taylor \\
\hline$p$ & Fator de penalidade das densidades intermediárias \\
\hline$t_{2}$ & Força fictícia aplicada no ponto B \\
\hline$d_{1}$ & Carga elétrica aplicada nos eletrodos da piezocerâmica \\
\hline$L_{t}(\mathbf{u}, \phi)$ & Forma bilinear da transdução média interna \\
\hline$L(\mathbf{u}, \phi)$ & Forma bilinear flexibilidade média interna \\
\hline$\rho_{i}$ & Densidade do elemento $i$ \\
\hline$\rho(x)$ & Função de distribuição contínua de densidades \\
\hline$\chi(x)$ & Função de distribuição discreta \\
\hline$f(x)$ & Função linear \\
\hline$F(x)$ & Função não linear \\
\hline$F$ & Função objetivo do problema de otimização \\
\hline$q$ & Função qualquer \\
\hline$t$ & Índice de transposição de matriz ou vetor \\
\hline
\end{tabular}




\begin{tabular}{|c|c|}
\hline$x_{\mathrm{i}}^{\min }$ & Limite móvel inferior da variável de projeto \\
\hline$x_{\mathrm{i}}^{\max }$ & Limite móvel superior da variável de projeto \\
\hline $\log$ & Logaritmo na base 10 \\
\hline $\ln$ & Logaritmo neperiano \\
\hline $\mathbf{N}$ & Matriz das funções de forma do elemento \\
\hline $\mathbf{N}_{u}$ & Matriz das funções de forma de deslocamento mecânico \\
\hline $\mathbf{N}_{\varphi}$ & Matriz das funções de forma de deslocamento elétrico \\
\hline B & Matriz de operadores diferenciais dos polinômios bilineares \\
\hline $\mathbf{C}$ & Matriz de parâmetros elásto-piezelétrica-dieléltrica \\
\hline c & Matriz de parâmetros elástico do material \\
\hline $\mathbf{K}$ & Matriz de rigidez global \\
\hline $\mathbf{J}$ & Matriz jacobiana \\
\hline$E_{0}$ & Módulo de elasticidade do material base \\
\hline$n v$ & Número de vizinhos do elemento central $i$ \\
\hline$\eta$ & Ordenada do sistema de coordenadas locais do elemento \\
\hline $\bar{w}_{f}$ & Peso atribuído heuristicamente \\
\hline$W_{f}$ & Peso da média ponderada \\
\hline$x$ & Posição \\
\hline$x_{0}$ & Posição inicial de partida para a PL \\
\hline $\mathrm{R}_{\max }$ & Raio de abrangência do filtro \\
\hline$v$ & Razão de Poisson \\
\hline$v_{0}$ & Razão de Poisson do material base \\
\hline$\Omega_{\mathrm{D}}$ & Região onde há presença de material \\
\hline$V^{*}$ & Restrição de volume de material \\
\hline $\mathbf{C}_{0}$ & Tensor constitutivo do material base \\
\hline $\mathbf{C}(x)$ & Tensor constitutivo do material isotrópico \\
\hline$\varepsilon$ & Tensor das deformações \\
\hline $\mathbf{T}$ & Tensor tensão \\
\hline $\mathbf{W}$ & Vetor deslocamentos \\
\hline
\end{tabular}




\begin{tabular}{ll}
$A_{n}$ & Variável de projeto qualquer \\
$\mathbf{v}$ & Vetor das componentes dos deslocamentos nodais \\
$\mathbf{t}_{k}$ & Vetor de carregamento de superfície \\
$\mathbf{u}$ & Vetor de deslocamentos \\
$\mathbf{n}$ & Vetor normal à superfície $\Gamma_{t^{k}}$ \\
$V_{e}$ & Volume do elemento \\
$V_{i}$ & Volume do elemento $i$ \\
$\Omega_{s}$ & Volume final de material desejado na estrutura a ser otimizada \\
$h_{e}$ & Espessura do elemento \\
$\mathbf{S}$ & Tensor elástico \\
$\mathbf{D}$ & Vetor deslocamento elétrico \\
$\mathbf{E}$ & Vetor campo elétrico \\
$\boldsymbol{E}_{D}$ & Tensor dielétrico \\
$\mathbf{e}$ & Tensor piezelétrico \\
$\mathbf{h}$ & Tensor campo elétrico/deformação \\
$\mathbf{d}$ & Tensor deformação/campo elétrico \\
$\mathbf{g}$ & Tensor campo elétrico/tensão mecânica \\
$\mathbf{\beta}$ & Tensor impermeabilidade dielétrica \\
$\boldsymbol{\phi}$ & Vetor deslocamento elétrico \\
$\mathbf{K}$ & Vetor potencial elétrico ou deslocamento elétrico \\
$\mathbf{K}$ & Vetor carga elétrica \\
$\mathbf{Q}$ & Matriz de rigidez \\
\hline$u \varphi$ &
\end{tabular}




\section{Capítulo 1: Atuadores Piezelétricos Flextensionais}

\subsection{Introdução}

Materiais piezelétricos possuem a propriedade de converter energia elétrica (campo elétrico e carga elétrica) em energia mecânica (força e deslocamento) e viceversa (RISTIK, 1983; IKEDA, 1996). São amplamente usados em sensores e atuadores eletromecânicos, acelerômetros e transdutores de ultrassom. Em engenharia os materiais piezelétricos usados são em geral cerâmicos (PZT) e polímeros (PVDF). Os atuadores piezelétricos são projetados para satisfazer os parâmetros de atuação exigidos durante o projeto, que é função do tipo de aplicação, como, por exemplo, garras, grampos, pinças, etc. Conseqüentemente, a solução para atender às condições de atuação, é conectar uma estrutura mecânica flexível (estrutura acoplada) na cerâmica (ou uma pilha - "stack" - de cerâmicas) para converter, direcionar e amplificar os pequenos deslocamentos gerados pela cerâmica piezelétrica (ordem de nanômetros). Essa montagem dá origem a um dispositivo denominado atuador piezelétrico flextensional (ROLT, 1990). Um exemplo desse tipo de transdutor é mostrado na Fig. 1.1.

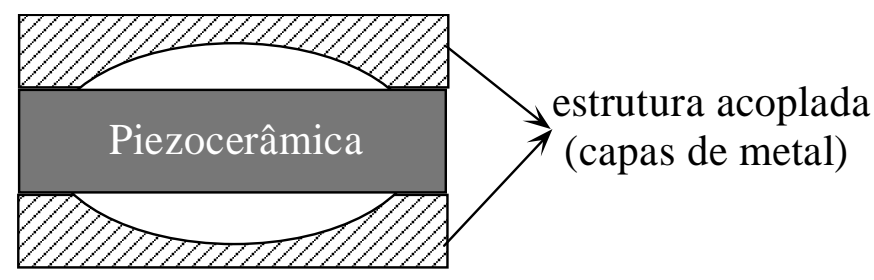

Figura 1.1- Atuador piezelétrico flextensional.

A estrutura flexível acoplada pode ser interpretada como um mecanismo 
flexível, ou seja, um mecanismo em que o movimento é dado pela flexibilidade da estrutura ao invés da presença de juntas e pinos. Dessa forma esses atuadores produzem deslocamentos mediante comandos de atuação através de sinais elétricos.

Esses atuadores flextensionais têm aplicação tradicional na atuação do avanço fino de ferramentas em máquinas $\mathrm{CNC}$ e grande aplicação em aparelhos que envolvem mecânica de precisão, como mecanismos de máquinas fotográficas, cabeçote leitor de um vídeo-cassete ou de um disco rígido de computador, onde devido à montagem compacta num pequeno espaço, exigem-se peças de pequenas dimensões e o menor número possível de componentes a serem montados (caso contrário o problema de folga na montagem pode inviabilizar o funcionamento do equipamento) e cuja atuação deve ser comandada por sinais elétricos.

Além disso, a maioria dos movimentos envolvidos nesses equipamentos deve ser realizada com pequenos deslocamentos precisos. Outra área de aplicação de atuadores piezelétricos flextensionais que tem surgido recentemente é na bioengenharia no projeto de instrumentos cirúrgicos eletromecânicos em cirurgias de laparoscopia, onde esses mecanismos podem ser usados na construção de microgarras, micropinças ou microtesouras acionadas por sinais elétricos (comandados pelo médico) que produzam movimentos variados (FUKUDA et al., 1995; CARROZZA et. al., 1998; MEHTA et al., 2002). Recentemente, outra área de potencial aplicação de atuadores piezelétricos flextensionais que tem emergido internacionalmente é a área de sistemas microeletromecânicos (ou "MEMS" em inglês). Nessa área, devido a microescala em que são fabricadas, a presença de pinos e juntas torna a montagem difícil senão impossível. Dessa forma, os atuadores em MEMS consistem essencialmente de mecanismos flexíveis, cuja atuação é realizada por efeito capacitivo, termoelétrico ou piezelétrico, comandados por sinais elétricos. Nesse caso, os MEMS consistiriam essencialmente em microatuadores piezelétricos flextensionais. As técnicas de fabricação de "MEMS" permitem a fabricação de qualquer estrutura plana de forma complexa. Vários artigos sobre o projeto e fabricação de atuadores para aplicação de "MEMS" podem ser encontrados na literatura (LARSEN; SIGMUND; BOUWSTRA, 1997; SIGMUND, 1998; SIGMUND, 1998c; JONSMANN; SIGMUND; BOUWSTRA, 1990, 1999a, 1999b). No Brasil, a fabricação de "MEMS" tem sido tema de interesse e estudo de grupos de 
pesquisa como o Laboratório de Sistemas Digitais (L.S.I.) do Departamento de Engenharia Eletrônica da Escola Politécnica da USP.

O projeto de atuadores piezelétricos flextensionais é uma tarefa muito complexa, pois essencialmente se deseja saber qual a geometria da estrutura flexível que deforme, gerando um dado deslocamento numa dada direção e ponto da estrutura, quando submetida a uma excitação elétrica nos eletrodos da cerâmica piezelétrica. No entanto, devido a esta complexidade, o projeto de atuadores piezelétricos flextensionais foi por algum tempo baseados em modelos analíticos simples, técnicas experimentais e métodos de otimização paramétrica. Porém, pesquisas recentes propõem procedimentos mais sistemáticos para o projeto, através do método de otimização topológica (SILVA; NISHIWAKI; KIKUCHI, 1999 e 2000).

Portanto, o objetivo deste trabalho é implementar um software para o projeto de atuadores piezelétricos flextensionais baseado no método de otimização topológica (MOT) que seja genérico e sistemático, podendo trazer grandes contribuições nessas áreas de aplicação. O MOT é implementado com o modelo de material do tipo SIMP (“Simple Isotropic Material with Penalization”) (BENDSØE, 1989; ZHOU; ROZVANY, 1991). Em trabalho anterior (SILVA; NISHIWAKI; KIKUCHI, 1999 e 2000) foi usado o método de homogeneização (MURAT; TARTAT, 1985; BENDSØE; KIKUCHI, 1988 e HASSANI; HILTON, 1998a, 1998 b e 1998c) como modelo de material.

Com isto, pretende-se projetar atuadores piezelétricos flextensionais aplicados em mecânica de precisão, em bioengenharia, MEMS, microrobôs, etc, utilizando o software implementado. O trabalho se limitará a princípio, ao projeto de atuadores flextensionais em duas dimensões, uma vez que a maior parte das aplicações envolve dispositivos bidimensionais, especialmente no caso de "MEMS" cujas técnicas de fabricação geralmente permitem implementar estruturas planas. 


\subsection{Histórico}

$\mathrm{O}$ projeto dos primeiros atuadores piezelétricos flextensionais eram baseados em modelos analíticos simples (XU et al., 1991a; XU et al., 1991b e DOGAN et al., 1994) e técnicas experimentais (DOGAN et al., 1994). A otimização desses transdutores piezelétricos limitava-se a otimizar dimensões específicas (por exemplo, a espessura da piezocerâmica). Estes modelos analíticos eram caracterizados por serem unidimensionais e aplicados somente a geometrias muito simples, sendo deficientes quando aplicados a transdutores com geometrias complexas. O nível de complexidade geométrica referia-se a formas de discos e paralelepípedos, e quando modelados não atendiam a precisão requerida no projeto (BRISSAUD, 1991). Portanto, os engenheiros eram limitados a transdutores de geometria simples, cujo desenvolvimento era baseado em modelos analíticos de construção de protótipos.

Como exemplo do primeiro transdutor piezelétrico flextensional tem-se o atuador piezelétrico "moonie", que é um sanduíche de um disco piezocerâmica entre "capas" de metal, cada uma das capas tem a forma de meia lua na cavidade interna da superfície (por isto o nome "moonie") (Fig. 1.2(a)), usada como transformador mecânico para converter e amplificar o deslocamento lateral da cerâmica em deslocamento axial maior na estrutura flexível (XU et al., 1991b). Os transdutores de "moonie" também são usados na geração de ondas ultrassônicas, como, por exemplo, em hidrofones (XU et al., 1991b; ONITSUKA et al., 1995). Os atuadores piezelétricos "moonie" foram caracterizados através de técnicas experimentais e modelos analíticos considerando como parâmetros, os deslocamentos, a força gerada e a freqüência de ressonância (XU et al., 1991a; XU et al., 1991b e DOGAN et al., 1994). 


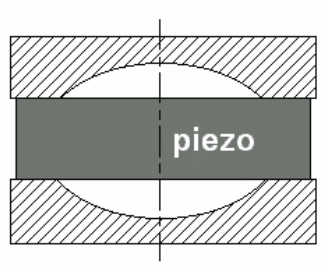

( a )

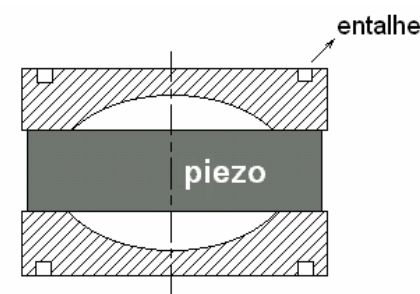

( b )

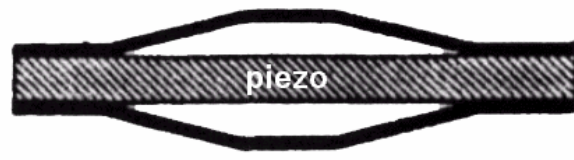

(c)

Figura 1.2- (a) Atuador "moonie"; (b) Atuador "moonie entalhado"; (c) Atuador "cymbal".

Os atuadores flextensionais possuem dois objetivos importantes de projeto, que são o deslocamento gerado e a força de blocagem (DOGAN; UCHINO; NEWNHAM, 1997). A força de blocagem é definida como sendo a máxima força suportada pelo atuador sem deformá-lo ou a força necessária para restaurar a forma não-deformada do atuador, para uma certa voltagem aplicada durante a atuação. $\mathrm{O}$ atuador "moonie" possui um desempenho versátil (combina um alto deslocamento gerado com uma força de blocagem moderada).

O grande avanço dos transdutores piezelétricos (e também flextensionais piezelétricos) foi possível com o desenvolvimento da teoria de elementos finitos piezelétrica desenvolvida por Allik; Hughes (1970). Com o MEF piezelétrico, podese modelar transdutores com geometrias complexas e iniciou-se a otimização do desempenho dos transdutores piezelétricos baseado na exploração de novas configurações. Assim, a construção de protótipos passou a ser necessária apenas para verificações dos resultados das estruturas piezelétricas, por exemplo, como sonares (NAILLON; COURSANT; BESNIER, 1983). A análise de estruturas piezelétricas foi implementada pela primeira vez num software comercial de MEF por Ostergaard; Pawlak (1986). As simulações passaram a ser usadas para otimizar dispositivos piezelétricos, tais como, transdutores ultra-sônicos para formação de imagens médicas (LERCH, 1990). No caso de atuadores piezelétricos flextensionais o MEF possibilitou otimizar o projeto do atuador "moonie" através da construção de curvas de desempenho dos atuadores considerando como parâmetros de otimização a freqüência de ressonância, o deslocamento e a força de blocagem (NEWNHAM et 
al., 1993; ONITSUKA et al., 1995). Portanto, o MEF piezelétrico, possibilitou substituir o método de otimização baseado na intuição do engenheiro por um método de otimização sistemático, conduzindo o problema a uma otimização paramétrica (CHALLANDE, 1990).

A otimização paramétrica do transdutor é obtida usando o MEF, para construir as curvas de deslocamento gerado e da força de blocagem como função de algumas dimensões de uma topologia específica, escolhida da estrutura acoplada. Esta escolha é usualmente baseada na intuição física do problema, como exemplo, o transdutor chamado "moonie entalhado" ("groove moonie") (Fig. 1.2(b)). Este transdutor foi obtido a partir de um estudo de concentração de tensões no transdutor "moonie", verificando-se que é possível eliminar parte da concentração de tensões removendo material da estrutura acoplada (na região onde apresentava a menor concentração de tensão), gerando ranhuras na estrutura. Neste caminho, uma variação do atuador "moonie entalhado", deu origem ao atuador "cymbal" (DOGAN; UCHINO; NEWNHAM, 1997) (Fig. 1.2(c)). Além disso, o entalhe aumenta a flexibilidade da parte exterior do acoplamento, provendo um deslocamento gerado maior do que o atuador "moonie" comum (Fig. 1.2(a)). No transdutor "cymbal", o deslocamento gerado é obtido pela combinação de movimentos de flexão e rotação, no entanto, os transdutores "moonie" comuns somente apresentam movimentos de flexão. Conseqüentemente, isto proporciona um deslocamento gerado maior do que o atuador "moonie" comum e também a força de blocagem são maiores devido ao aumento da rigidez axial.

Portanto, no passado o desenvolvimento desses transdutores eram através da otimização paramétrica, e como visto, o desempenho dos atuadores piezelétricos são medidos pelos deslocamentos gerados e pela força de blocagem. Esses estudos mostram que grandes deslocamentos e uma alta força de blocagem são soluções de compromisso, e dependem da rigidez e flexibilidade da estrutura acoplada na cerâmica piezelétrica. Dessa forma, no projeto da estrutura acoplada deve-se considerar a distribuição de rigidez e flexibilidade, o que pode ser obtido usando otimização topológica. Assim, projetando-se diferentes tipos de estruturas acopladas, novos tipos de transdutores piezelétricos flextensionais são obtidos, com desempenho variados e que podem ser aplicados a diferentes tarefas. 
O método de Otimização Topológica (BENDSØE; KIKUCHI, 1988; BENDSØE, 1989 e SUZUKI; KIKUCHI, 1991) que originalmente foi desenvolvido para projetos de maximização da rigidez de estruturas, tem sido usado recentemente com sucesso no projeto de mecanismos flexíveis (ANANTHASURESH; KOTA; GIANCHANDANI, 1994; ANANTHASURESH; KOTA, 1995). Frecker et al. (1997) e Nishiwaki et al. (1998) apresentaram uma formulação multi-objetivo, no qual se maximiza a razão entre a energia mútua e a energia de deformação (flexibilidade média), para mecanismos flexíveis. Assim, passou a ter uma formulação que atende às exigências de estabilidade estrutural considerando a energia mútua (EM) e a flexibilidade média (FM). Silva; Nishiwaki; Kikuchi (1999, 2000) expandiram os conceitos de EM e FM aplicada a mecanismos flexíveis para atuadores piezelétricos flextensionais, desenvolvendo um método que permite projetar vários tipos de atuadores piezelétricos não convencionais, como, por exemplo, garras, grampos, pinças, etc... Por ser genérica e sistemática a OT tem obtido grande sucesso e aceitação no projeto de atuadores piezelétricos flextensionais. A partir deste trabalho, foi iniciado o estudo de parâmetros que influenciam na formulação do MOT aplicado a atuadores piezelétricos e a construção de protótipos, como, por exemplo, Canfield; Frecker (2000) estudaram a formulação do problema de OT e o método de solução (Programação Linear Seqüencial e Critério da Optimalidade) num domínio discretizado com elementos do tipo treliça, os resultados obtidos foram verificados usando o MEF. Na sequiência, Frecker; Calfield (2000) projetaram atuadores piezelétricos com elementos de treliça e sólidos, verificando o desempenho destes atuadores piezelétricos através do MEF e experimentalmente, o que possibilitou estudar o efeito de parâmetros que influem no desempenho (como as juntas). Em outros estudos, passaram a projetar atuadores piezelétricos considerando condições especiais, como, por exemplo, obter a estrutura flexível que forneça a máxima amplificação dos deslocamentos gerados pela piezocerâmica ou pilhas piezocerâmicas, usando funções objetivo específicas adequadas para cada tipo de problema (LAU; DU; LIM, 2000). Com a construção de modelos sólidos, pode-se verificar o desempenho destes modelos e a influência das juntas e de outros parâmetros (FRECKER; CANFIELD, 2000).

Contudo, ainda há muitos projetos de atuadores piezelétricos sendo 
desenvolvidos sem a utilização de um método sistemático em diversas áreas de atuação, como, por exemplo, o projeto de um atuador piezelétrico atuando como um freio de tambor (GOGOLA; GOLDFARB, 1999); projeto do controle de um nanoposicionador de uma rede neural em tempo real, desenvolvido por $\mathrm{Ku}$ et al. (2000). Na bioengenharia há muitos instrumentos que podem ser inovados usando atuadores piezelétricos flextensionais projetados pelo MOT, como, por exemplo, manipuladores atuados piezeletricamente, que são aplicados na manipulação de micro-objetos biológicos (CARROZZA et al., 1998); dispositivos de atuação de micro-máquinas, principalmente na área médica, que pode ser aplicado em cirurgias delicadas para evitar incisões desnecessárias (FUKUDA et al., 1995). Neste sentido, visando aperfeiçoar os instrumentos atuados piezeletricamente, Mehta et al. (2002), começou a analisar o desempenho dos instrumentos aplicados em laparoscopia, com o intuito de aperfeiçoar estes instrumentos buscando melhorar a eficiência cirúrgica.

\subsection{Justificativa}

Como já comentado, atuadores piezelétricos flextensionais têm grande aplicação em diversas áreas, constituindo tema de interesse e estudo de grupos de pesquisa no Brasil.

Devido a sua aplicação multidisciplinar, o projeto desses atuadores piezelétricos exige que os profissionais de diferentes áreas adquiram conhecimentos específicos sobre estruturas, materiais piezelétricos e projeto de atuadores, o que de certa forma limita a sua aplicação nessas várias áreas. Dessa forma, a implementação de um software para o projeto de atuadores piezelétricos flextensionais que seja genérica e sistemática, poderá trazer grandes contribuições nessas áreas de aplicação. Além disso, o software permitirá uma maior divulgação da tecnologia de atuadores piezelétricos flextensionais no meio acadêmico, principalmente, alunos de graduação e pós-graduação em engenharia.

O método de otimização topológica no projeto de atuadores piezelétricos flextensionais encontra-se bem difundido na comunidade científica da área nos 
Estados Unidos e Europa, sendo atualmente desenvolvido pelo orientador do candidato (Prof. Dr. Emílio Carlos Nelli Silva). Dessa forma esse trabalho de mestrado procura difundir esse tema no Brasil. 


\section{Capítulo 2: Otimização Topológica}

\subsection{Introdução}

Otimização topológica consiste num método computacional que permite projetar a topologia ótima de estruturas segundo um certo critério de custo (por exemplo, máxima rigidez e menor peso). Basicamente, o MOT distribui o material no interior de um domínio fixo de forma a maximizar ou minimizar uma função custo especificada. O material em cada ponto do domínio pode variar de ar (não há presença de material) até sólido (total presença de material) podendo assumir densidades intermediárias entre ar e sólido de acordo com um modelo de material definido.

Um algoritmo de otimização é usado para encontrar de forma iterativa a distribuição ótima de material, o que torna o processo rápido, caso contrário milhões de análises seriam necessárias para encontrar a distribuição ótima. Na aceleração do processo de busca da distribuição ótima de material, os métodos de otimização utilizam-se da informação dos gradientes (ou derivadas) da função custo em relação à quantidade de material em cada elemento. A distribuição de material é representada, por exemplo, associando um valor de densidade a cada elemento (subdomínio), obtido da discretização do domínio inicial.

Dessa forma, a OT combina essencialmente métodos de otimização (VANDERPLATZ, 1984; HAFTKA; GURDAL; KAMAT, 1996) com um método numérico de análise, como por exemplo, o método de elementos finitos (MEF) (NAILLON; COURSANT; BESNIER, 1983; LERCH, 1990 e BATHE, 1996). Outros métodos numéricos de análise podem ser usados, no entanto, devem ser genéricos o suficiente de forma a lidar com estruturas de formas complexas resultantes da otimização.

O MOT é um tema recente no campo da otimização estrutural. Porém, os 
conceitos básicos que dão suporte teórico ao método foram estabelecidos a mais de um século. Rozvany; Bendsøe; Kikuchi (1995) fazem uma boa revisão desses conceitos. Para entender melhor como surgiu o método e como ele se estabeleceu atualmente nos projetos de estruturas mecânicas, devemos antes conhecer as diferentes formas de otimização estrutural existentes. A Fig. 2.1 ilustra dois tipos de otimização, como mostrado abaixo:

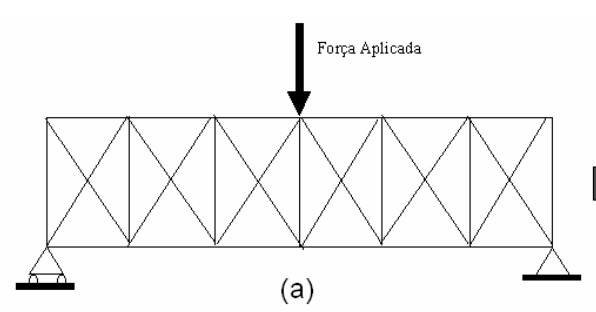

(a)

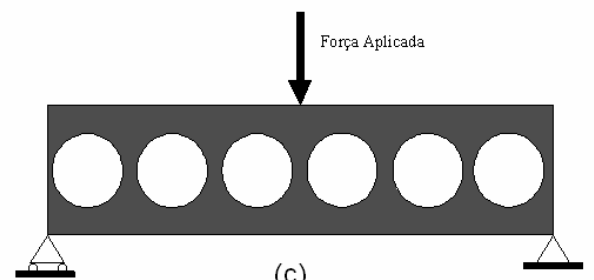

(c)

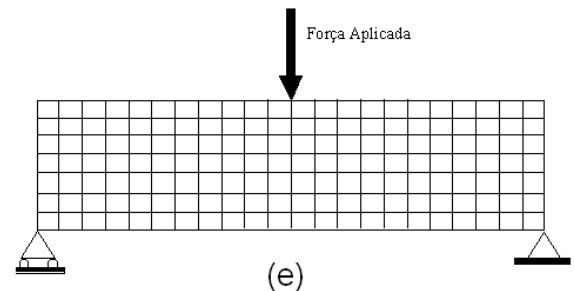

(e)

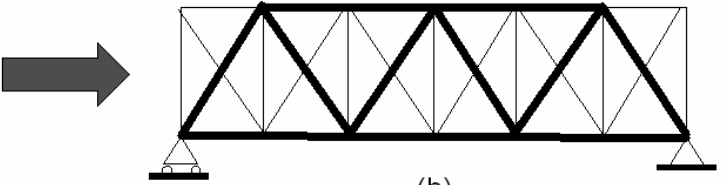

(b)

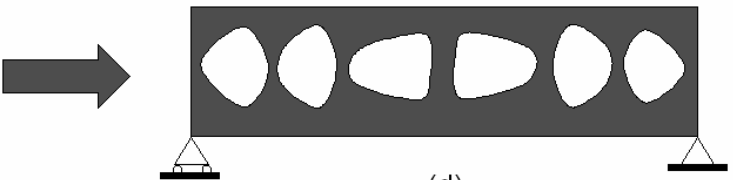

(d)

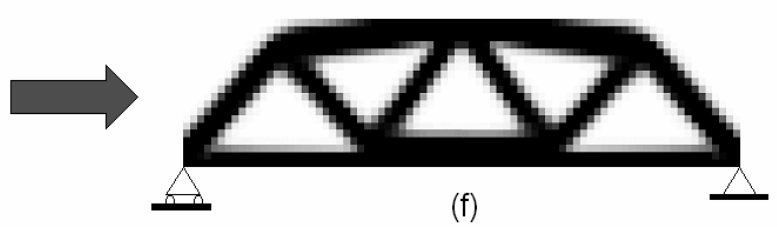

Figura 2.1 - Exemplo de 3 categorias de otimização estrutural: (a) otimização paramétrica; (c) otimização de forma e (e) otimização topológica.

Basicamente a otimização de estruturas mecânicas busca a melhor configuração possível de maneira a atender uma função objetivo especificada. Dentro do projeto é necessário selecionar qual a melhor maneira de se obter a ótima configuração da estrutura. A primeira categoria de otimização estrutural (Fig. 2.1(a)) consiste em assumir para a estrutura uma forma fixa previamente definida, neste caso, a estrutura está discretizada com elementos de treliça, onde as características 
geométricas, como, por exemplo, a área da secção transversal de cada elemento (Fig. 2.1(a)) são as variáveis de projeto do problema, ou seja, os parâmetros que podem ser alterados para otimizar a estrutura. Essa abordagem é conhecida por otimização paramétrica (ou "size optimization" como é conhecida na literatura internacional). Assim, utilizando um algoritmo computacional de otimização para encontrar as áreas individuais de cada elemento de treliça, que maximiza a rigidez da estrutura respeitando uma determinada restrição (por exemplo, volume final da estrutura) (Fig. 2.1(b)). A segunda categoria é a otimização de forma (ou "shape optimization"), onde os contornos externos e internos da estrutura são parametrizados por curvas "splines" e os parâmetros dessas curvas constituem as variáveis de projeto (Fig. 2.1(c)). Daí, através de um algoritmo computacional de otimização são determinados os parâmetros ótimos das curvas "splines", e, portanto a forma ótima da estrutura (Fig. 2.1(d)) que otimiza a função custo do projeto. A última categoria consiste em se obter a configuração ótima, buscando encontrar a distribuição ótima de material no interior da estrutura (Fig. 2.1(e)), de tal forma a permitir a criação de "cavidades" nas regiões onde não houver a necessidade de material (Fig. 2.1(f)), observando que a estrutura resultante deve otimizar a função custo do projeto. Essa abordagem é chamada de otimização topológica (ou "topology optimization”). As variáveis de projeto podem ser, por exemplo, medidas que indicam a distribuição de material em cada ponto do domínio. A otimização topológica é a mais genérica, sendo que a quantidade de material removida (para a mesma função custo) é maior do que nos outros métodos de otimização, conseqüentemente, obtêm-se a estrutura mais leve e com melhor desempenho.

\subsection{Histórico}

A otimização topológica surgiu da necessidade de se aprimorar a otimização de forma para permitir a viabilidade de uma distribuição de material mais acentuada no interior do domínio da estrutura, ou seja, a otimização de forma permite a mudança no contorno interno e externo da estrutura, no entanto, não permite inserir novos "buracos" no corpo da estrutura para reduzir o seu peso, sem violar as 
restrições de projeto.

No final da década de 80, Bendsøe e Kikuchi (BENDSØE; KIKUCHI, 1988) introduziram uma metodologia alternativa para a otimização de forma, que parte do princípio de se fixar o domínio inicial da estrutura, e, portanto manter inalterado o modelo de elementos finitos utilizado no processo de otimização. Desta maneira, surge a metodologia para otimização topológica de estruturas mecânicas, baseado no conceito de domínio fixo estendido de projeto, e, inicialmente, no método da homogeneização (BENDSØE; KIKUCHI, 1988) (ver item 2.5.2).

A metodologia criada por Bendsøe; Kikuchi (1988) para a OT foi baseada nos trabalhos de otimização da distribuição de espessuras em placas e chapas (CHENG; OLHOFF, 1981, 1982) e de barras de torção projetadas com dois materiais em diferentes proporções volumétricas (LURIE; FEDEROV; CHERKAEV, 1982; GOODMAN; KOHN; REYNA, 1986 e KOHN; STRANG, 1986a). Na formulação matemática para o problema de maximização de rigidez (com restrição de volume) de placas delgadas, onde a variável de projeto é a espessura da placa, concluíu-se que existem várias soluções ótimas locais (CHENG; OLHOFF, 1981). Rozvany et al. (1982) também chegaram na mesma conclusão. Ou seja, os resultados encontrados por eles indicam que a solução ótima é uma placa que, ao longo da espessura, é composta por regiões com infinitos reforços infinitesimais (nervuras), cujo comportamento é similar a um material composto por infinitas microestruturas. Em termos matemáticos a introdução de uma microestrutura na formulação de um problema estrutural requer a relaxação do funcional (variação da função) do problema para permitir a otimização. Detalhes do cálculo da relaxação dos funcionais na otimização estrutural podem ser encontrados em KOHN; STRANG (1986a, 1986b). Bendsøe (1989) descreve várias maneiras de se conseguir a relaxação mencionada acima através da introdução de um modelo de material baseado numa lei de mistura de materiais na microestrutura, dentre eles o chamado método de densidades. O modelo de material que será descrito com mais detalhes no item 2.6, permite que a natureza discreta da função de distribuição de material seja aproximada por uma de natureza contínua, ou seja, o problema de otimização é definido de tal forma que os parâmetros desse modelo de material sejam as variáveis de projeto, sendo que essas agora são descritas por funções contínuas que permitem 
estágios intermediários ao se passar de uma condição extrema para outra (por exemplo, sólido para "vazio"). Isso garante a relaxação do problema evitando mudanças bruscas de material ao longo das iterações de otimização.

As microestruturas constituem a base da OT e podem ser definidas através do modelo de material como será visto no item 2.5.2. Uma revisão sobre as características das microestruturas utilizadas no Método de Otimização Topológica (MOT) pode ser vista em Hassani; Hinton (1998b). A metodologia para OT foi definida em Bendsøe; Kikuchi (1988) e Suzuki; Kikuchi (1991), que implementaram o método para resolver, com sucesso, vários exemplos de otimização estrutural cuja função objetivo do problema era a maximização de rigidez, sujeita à restrição de volume de material. Dessa forma, o MOT passou a ser utilizado em diferentes aplicações, como, os citados a seguir.

Bendsøe; Rodrigues (1991) e Olhoff; Bendsøe; Rasmussen (1991) estudaram a possibilidade da integração do MOT aos sistemas de CAD (desenho assistido por computador). Díaz; Bendsøe (1992) apresentaram uma formulação para o problema de maximização de rigidez de estruturas elásticas submetidas a várias cargas não simultâneas. Thomsen (1992) e Olhoff; Kog; Thomsen (1993) trataram numericamente uma extensão do método aplicada a otimização de estruturas compostas por mais de um material. Outra extensão do método para aplicação em projeto de reforçamento de chapas de carrocerias de automóveis pode ser encontrada em Fukushima; Suzuki; Kikuchi (1993). Problemas de OT considerando frequiência de ressonância em estruturas contínuas são descritos em Díaz; Kikuchi (1992) e Ma; Kikuchi; Hagiwara (1993). Além das aplicações na área estrutural clássica, pode-se citar, por exemplo, a aplicação do método por Ananthasuresh; Kota; Gianchandani (1994) em projetos de mecanismos flexíveis motivou o surgimento de vários trabalhos nesta área (SIGMUND, 1996, LARSEN; SIGMUND; BOWSTRAM, 1997; NISHIWAKI et al., 1998, entre outros). Problemas de maximização da condutividade térmica na transferência de calor são resolvidos em Park (1995).

Atualmente a metodologia da OT está bem estabelecida, expandiu-se para outras áreas de projeto. Dentre eles, projeto de micromecanismos flexíveis com atuação térmica (SIGMUND, 1999, JONSMANN; SIGMUND; BOUWSTRA, 
1999), projetos de estruturas sob atuação de campos magnéticos (YOO; KIKUCHI, 2000), implementação de flexibilidade com carga dinâmica (NISHIWAKI et al., 2000), entre outros.

\subsection{Procedimento para implementação da Otimização Topológica}

A metodologia criada para a OT gera um procedimento constituído de várias etapas para a obtenção da solução ótima. (BENDSØE; KIKUCHI, 1988 e SUZUKI; KIKUCHI, 1991). É importante observar que no processo de solução do problema de otimização o domínio da estrutura é discretizado, portanto torna-se necessário à utilização de métodos numéricos.

As etapas para o projeto de estruturas mecânicas utilizando o MOT, é apresentado na Fig. 2.2. A primeira etapa consiste em se definir o domínio estendido fixo de projeto $(\Omega)$, no qual a estrutura pode existir. Esse domínio é limitado pelos pontos de aplicação de carga e pelas condições de contorno da estrutura, ou seja, os pontos onde os deslocamentos são restringidos. É importante nessa fase definir o domínio $(\Omega)$ com o maior tamanho possível, de forma a não limitar o domínio de trabalho da Otimização Topológica, pois como em qualquer método de otimização quanto mais restringimos o problema, reduzimos o espaço de soluções. Na segunda etapa o domínio $(\Omega)$ é discretizado em elementos finitos e são aplicadas as condições de contorno. Na terceira etapa, os dados do domínio $(\Omega$ ) são inseridos no algoritmo de otimização topológica, que num processo iterativo distribui o material no domínio $(\Omega)$ de forma a minimizar (ou maximizar) a função objetivo especificada. O resultado obtido é a topologia ótima mostrada na Fig. 2.2 (item "Resultado da OT"), onde a cor escura indica a presença de material e a cor branca indica a ausência de material no ponto do domínio.( $\Omega$ ). Note que podem surgir pontos com cores intermediárias, denominados de escalas de cinza ("gray scale"). Essas cores indicam a presença de materiais intermediários no interior do domínio.( $\Omega$ ), os quais são difíceis de serem implementados na prática, no entanto são inerentes ao problema de Otimização Topológica (BENDSØE, 1989 e SUZUKI; 
KIKUCHI, 1991). Outro problema é a "instabilidade xadrez", que surge devido as regiões com elementos de cor escura e elementos de cor branca dispostos em forma de tabuleiro. A rigidez da região que contém a "instabilidade xadrez" é maior se comparada à rigidez de uma região com distribuição uniforme de material. Maiores detalhes da "instabilidade xadrez" podem ser observados no item 2.7.

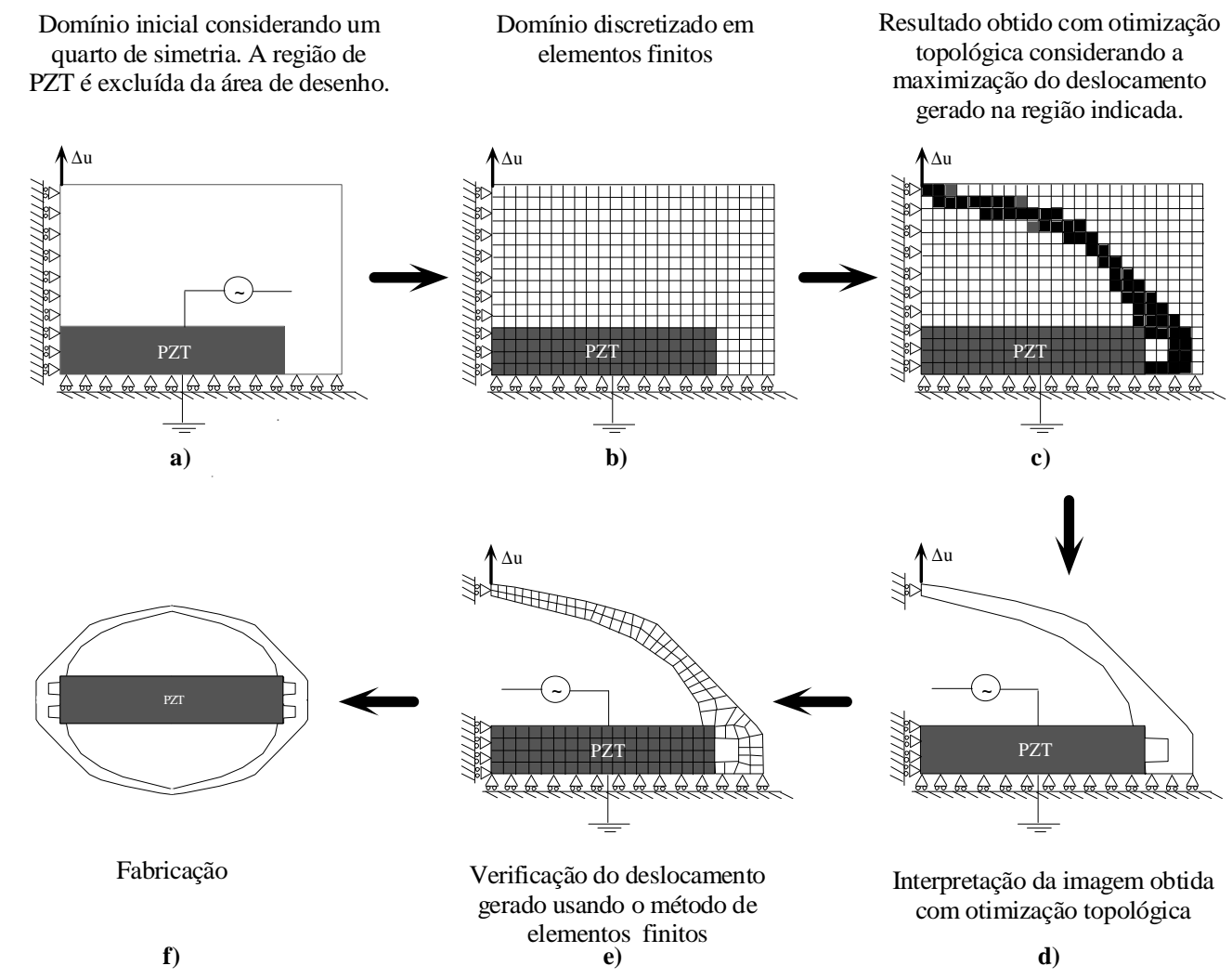

Figura 2.2 - Procedimento típico de projeto estrutural por otimização topológica.

Portanto, as "escalas de cinza" e a "instabilidade xadrez" são indesejáveis e irão prejudicar a interpretação dos resultados da otimização (quarta etapa). Essa interpretação consiste na remoção das eventuais "escalas de cinza" e a suavização do contorno da topologia obtida, ajustando-a de forma que possa ser fabricada. Existem várias técnicas usadas para realizar a interpretação dos resultados, como desde desenhar uma estrutura baseada na topologia obtida até o processamento de imagens (KIKUCHI; HOLLISTER; YOO, 1997 e HSU; HSU; CHEN, 2001). A quinta etapa 
consiste em se verificar o resultado final da estrutura, com o objetivo de avaliar a alteração da função objetivo devido às pequenas alterações da interpretação. Além disso, os resultados gerados na Otimização Topológica não são intuitivos e é interessante fazer uma verificação da estrutura final usando o MEF (após a interpretação da topologia). Finalmente, a última etapa é a fabricação da estrutura onde, atualmente, existem várias técnicas de fabricação que permitem obter estruturas com formas complexas, como, a prototipagem rápida, a eletro-erosão a fio, processo de corrosão química pelo método de litografia, entre outras.

\subsection{Aspectos da otimização topológica}

A OT de estruturas pode ser dividida em duas categorias distintas: a otimização topológica a partir de um meio discreto (itens 2.4.1 e 2.4.2) e a otimização topológica a partir de um meio contínuo (item 2.4.3). A OT a partir de um meio discreto considera o domínio contínuo da estrutura discretizado por barras rígidas ou treliças, como mostrado na Fig. $2.4 \mathrm{~b}$, sendo subdividida em outras duas categorias (ROZVANY; BENDSØE; KIRSCH, 1995), como mostrado nos subitens 2.4.1 e 2.4.2.

\subsubsection{Otimização topológica de estruturas de malha contínua}

A OT de estruturas de malha contínua trabalha com um domínio de projeto com infinito número de barras rígidas separadas por um espaço infinitesimal, cuja solução ótima é obtida analiticamente através da teoria da elasticidade. Este conceito de otimização foi desenvolvido em 1904 por Michell. Michell foi o pioneiro a oferecer uma abordagem teórica para a solução analítica de problemas de otimização em estruturas de malha contínua com mínimo volume de material. A Fig. 2.3 mostra um exemplo da configuração otimizada de uma alavanca encontrada por Michell onde as linhas de isotensão principais, determinadas através da teoria da elasticidade, 
podem ser compostas por uma série de barras rígidas.

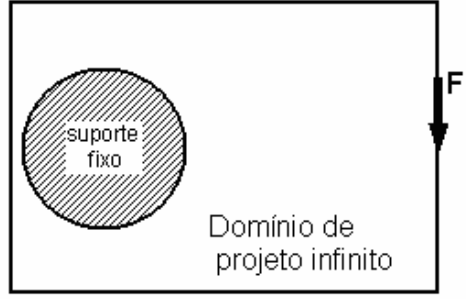

a)

Problema de otimização estrutural

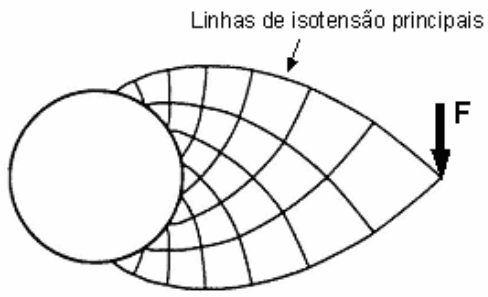

b)

Resultado analítico obtido por Michell em 1904

Figura 2.3. Otimização estrutura obtida por Michell.

Com o advento dos computadores e o desenvolvimento dos métodos numéricos, os trabalhos de Michell foram retomados por Hemp (1973) e Prager (1974) e recentemente surgiram outras extensões desses trabalhos (ZHOU; ROZVANY, 1991 e BENDSØE; BEN-TAL; ZOWE, 1994).

\subsubsection{Otimização topológica de estruturas discretas}

A OT de estruturas discretas considera um domínio de projeto onde há vários pontos distribuídos e que podem ser uma possível junta (ou ligação) de treliças (DORN, 1964 apud ROZVANY; BENDSØE; KIKUCHI, 1995), onde a solução ótima é obtida numericamente. Há vários métodos numéricos para a otimização de estruturas discretas, dentre eles a programação linear (YANG; CHUANG, 1994), o critério de optimalidade (ROZVANY; ZHOU, 1991 e ZHOU; ROZVANY, 1991) são considerados os mais adequados para analisar grandes estruturas, compostas por milhares de barras rígidas (treliças). A análise de otimização elimina as treliças desnecessárias à estrutura, onde a topologia final obtida é dada por elementos de treliça, cuja variável de projeto (área da seção transversal ou coordenada dos nós da 
treliça) são otimizadas de forma a atender uma função custo especificada, por exemplo, minimizar a flexibilidade da estrutura (função objetivo) sujeita a restrição de volume de material (função custo) como na estrutura discreta ilustrada na Fig. 2.4. O desenvolvimento deste conceito pode ser estendido para outras áreas além da área estrutural clássica, como, por exemplo, no projeto de mecanismos flexíveis (FRECKER; KIKUCHI; KOTA, 1996) e atuadores piezelétricos flextensionais (CALFIELD; FRECKER, 2000).

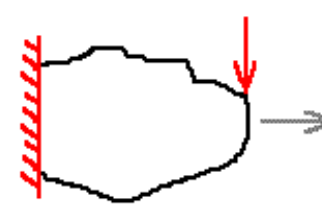

a)

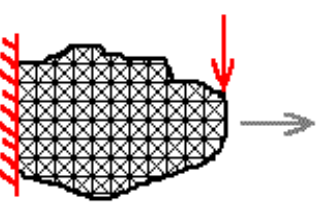

b)

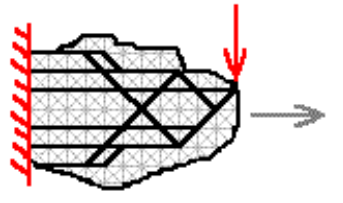

c)

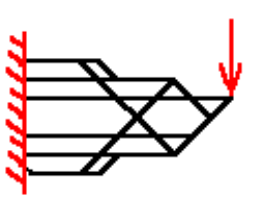

d)

Figura 2.4 - Otimização topológica de uma estrutura discreta.

\subsubsection{Otimização topológica de um meio contínuo}

Finalmente, a otimização topológica a partir de um meio contínuo, onde se busca a topologia final ótima através do MOT. Os trabalhos desenvolvidos usando o MOT (BENDSØE; KIKUCHI, 1988) mostram um método eficiente na busca da ótima configuração estrutural (objetivo do MOT), inclusive Suzuki; Kikuchi (1991) reproduziram o resultado analítico obtido por Michell (Fig. 2.3b), para o problema estrutural mostrado na Fig. 2.5, comprovando o embasamento matemático do método. 


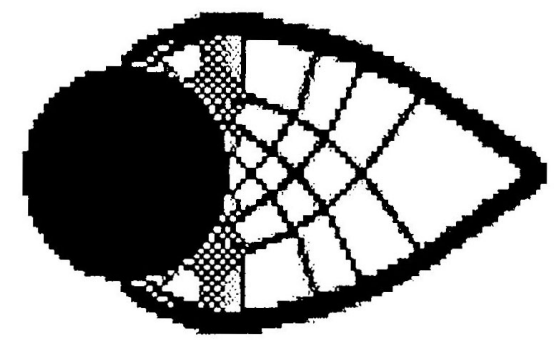

Figura 2.5- Resultado da Otimização Topológica do problema resolvido anteriormente por Michell.

\subsection{Conceitos do MOT}

O MOT é baseado em dois conceitos: domínio estendido fixo de projeto (Fig. 2.6) e modelo de material, que são descritos nos subitens seguintes.

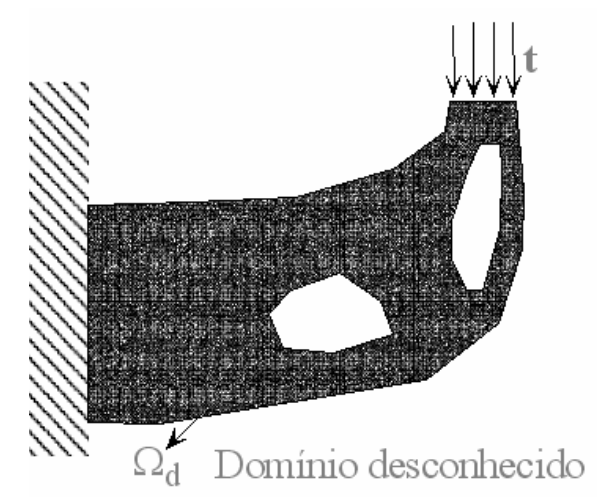

(a)

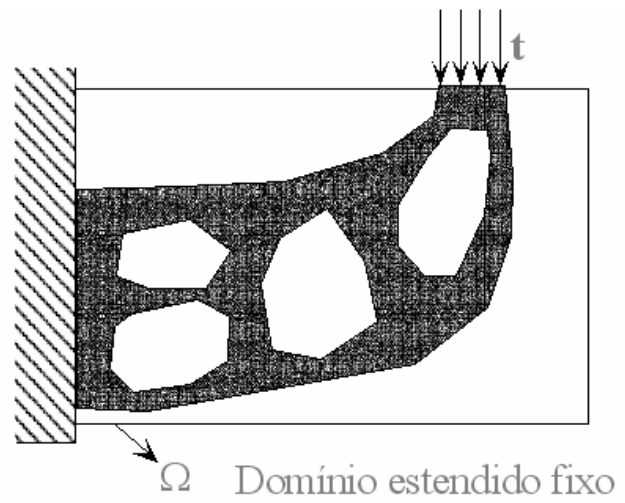

(b)

Figura 2.6- Conceitos de domínios para otimização de forma (a) e topológica (b).

Como descrito no item 2.1, na otimização de forma parte-se de um domínio desconhecido, onde os contornos externos e internos da estrutura são parametrizados por curvas "splines" e os parâmetros dessas curvas constituem as variáveis de 
projeto, no qual, o algoritmo computacional de otimização determina a forma ótima da estrutura (Fig. 2.6(a)).

\subsubsection{Domínio estendido fixo de projeto}

O domínio estendido fixo de projeto $(\Omega$ ) é o espaço no qual o algoritmo de otimização topológica pode construir a estrutura (Fig. 2.6(b)). Consiste em um domínio de forma fixa, limitado pelos pontos de apoio da estrutura e pontos de aplicação de carregamento, o que influencia no projeto da estrutura. Ou seja, num problema de OT a forma ótima é determinada sem quaisquer especificações geométricas, sendo influenciada pela quantidade de material utilizada, os pontos de fixação (restrição de deslocamento) e aplicação de cargas. O objetivo da Otimização Topológica é determinar os espaços sem material ou "vazio" (variável de projeto) e a conectividade da estrutura através da remoção e adição de material nesse domínio de forma a extremizar uma função objetivo. O problema de otimização consiste, portanto em se encontrar a distribuição ótima de propriedades de materiais no domínio estendido fixo. Na implementação numérica o domínio estendido fixo é discretizado em elementos finitos. Portanto, o modelo de elementos finitos do domínio não é alterado durante o processo de otimização, sendo alterado somente a sua distribuição de material nos elementos. O que torna o processo de otimização bastante vantajoso, pois sendo o domínio fixo, as derivadas de qualquer função $q$ são facilmente calculadas usando a expressão:

$$
\frac{\partial}{\partial A_{n}} \int_{\Omega} q d \Omega=\int_{\Omega} \frac{\partial q}{\partial A_{n}} d \Omega
$$

onde $A_{n}$ é uma variável de projeto, e $q$ é uma função qualquer definida no domínio. 


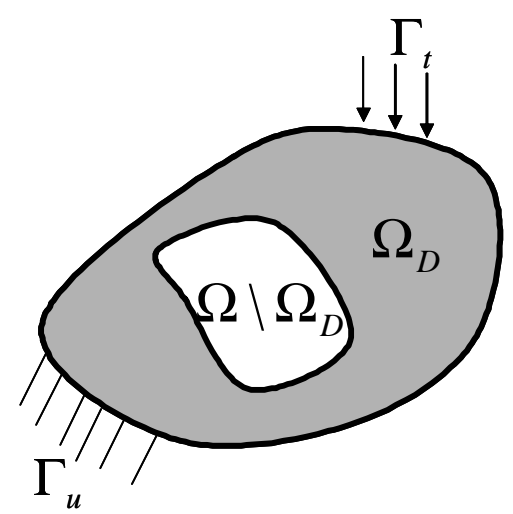

Figura 2.7- Definição do domínio de projeto e das condições de contorno.

\subsubsection{Modelo de material}

O modelo de material é uma equação que define a mistura em micro-escala de dois ou mais materiais (um deles pode ser "vazio") permitindo que hajam estágios intermediários ao se passar da condição de zero material ("buraco") a sólido em cada ponto do domínio.

De forma básica, a estrutura a ser otimizada pode ser definida por uma função discreta $\chi(\mathrm{x})$, definida em cada ponto $(\mathrm{x})$ do domínio $(\Omega)$, da seguinte maneira:

$$
\chi(\mathrm{x})=\left\{\begin{array}{lll}
1 & \text { se } & \mathrm{x} \in \Omega_{\mathrm{D}} \\
0 & \text { se } & \mathrm{x} \in \Omega \backslash \Omega_{\mathrm{D}}
\end{array}\right.
$$

onde $\Omega_{\mathrm{D}}$ é a região onde há presença de material, inserida no domínio ( $\Omega$ ) (ver Fig. 2.7). Sendo o material isotrópico, podemos escrever:

$$
\mathbf{C}(\mathrm{x})=\chi(\mathrm{x}) \mathbf{C}_{0}
$$


onde $\mathbf{C}_{0}$ é o tensor constitutivo do material base. Ou seja, fisicamente a função discreta $\chi(x)$ define se o ponto $(x)$ do domínio é preenchido totalmente com material (sólido) ou é um vazio (buraco), não havendo estágios intermediários.

Mas, a parametrização discreta da Eq. (2.3) apresenta duas dificuldades importantes, que são:

- As variáveis de projeto são variáveis binárias que indicam a presença ou ausência de material. Para resolver este problema é necessário recorrer a um método de otimização em variáveis inteiras. No entanto, é necessário discretizar o domínio em milhares de elementos para se obter uma solução realista e os métodos padrões de otimização em variáveis discretas apresentam desvantagens na solução de problemas de tais dimensões.

- A parametrização discreta da Eq. (2.3) tende a criar microestruturas à medida que refinamos a malha, e conseqüentemente melhor será a representação da microestrutura, o que gera a dependência da discretização e a não-unicidade da solução para o problema discretizado (BENDSØE, 1995). Ou seja, quanto mais refinarmos a discretização do domínio, a solução tende a conter regiões com alternância de sólido e vazio ( 0 ou 1$)$. Portanto, a solução para este problema pode ser encontrada na relaxação da variável de projeto.

Uma maneira de relaxar o problema, ou seja, permitir que as variáveis de projeto assumam valores intermediários entre 0 e 1, é definir um modelo de material substituindo a função discreta por uma contínua (CHENG; OLHOFF, 1982; BENDSØE, 1989). A princípio, os estágios intermediários não têm significado físico sendo apenas decorrentes de um recurso matemático para relaxação do problema. Segundo Bendsøe (1995), um modelo de material que fornecer uma função contínua e consistente das propriedades do material em cada ponto do domínio, garante o alcance da solução. Existem vários modelos de material que podem ser utilizados, entre eles o método de densidades (BENDSØE, 1989; ZHOU; ROZVANY, 1991; MLEJNEK, 1992) e o método da homogeneização (MURAT; TARTAR, 1985; BENDSØE; KIKUCHI, 1988). Neste item será feita uma breve descrição sobre o método da homogeneização. No presente trabalho é utilizado o método de densidades, cujo detalhamento é feito no item 2.6. 
O método da homogeneização é baseado em microestruturas formadas pela mistura de materiais homogêneos (MURAT; TARTAR, 1985, BENDSØE; KIKUCHI, 1988), uma revisão sobre esse método pode ser encontrada em Hassani; Hinton (1998a). Esse método se constitui num modelo de material complexo e robusto para a definição das propriedades efetivas de um material composto, conhecida a geometria e composição de sua microestrutura. Assim, tomando-se como exemplo uma placa perfurada podemos calcular as propriedades da composição dos materiais da placa perfurada (sólido + "vazio") a partir do material base da placa e conhecendo a distribuição dos furos na mesma. No MOT, cada ponto do domínio da estrutura é definido como sendo um material composto gerado pela repetição periódica de uma microestrutura. Desta maneira, existem duas configurações de microestrutura que podem ser utilizadas, a partir das quais podem ser geradas outras microestruturas (FUJII et al., 2001). Uma delas é a microestrutura composta por material sólido com vazio interno (célula 1 na Fig. 2.8) e a outra é a microestrutura composta por camadas alternadas de material e vazio (célula 2 na Fig. 2.8).

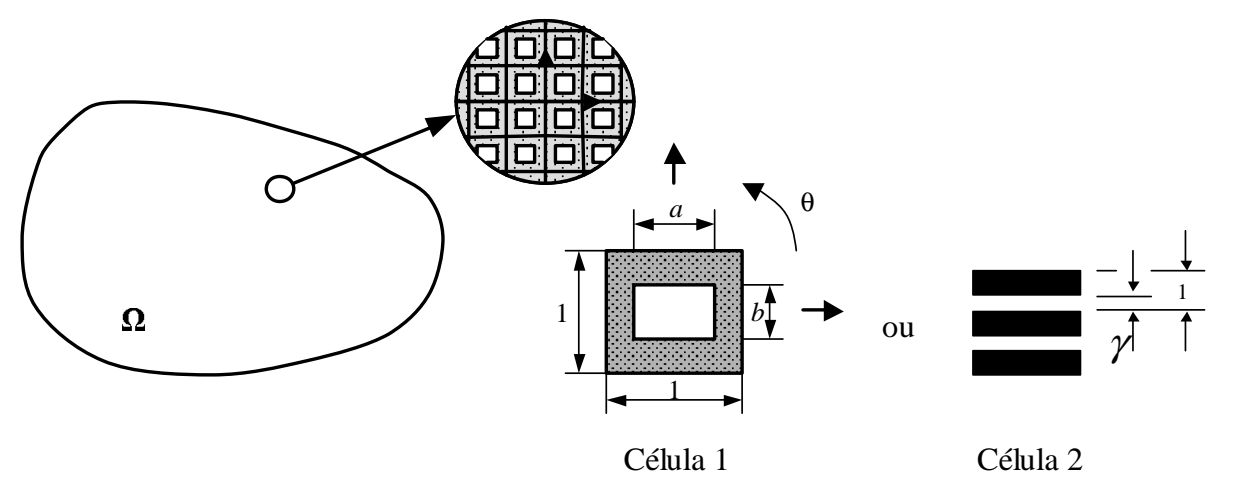

Figura 2.8 - Microestruturas para o método da homogeneização.

A microestrutura composta por camadas alternadas de material (célula 2 na Fig. 2.8) é construída alternando-se camadas de materiais sólidos com "vazios" e cujo parâmetro de otimização é a medida $\gamma$. THOMSEN (1992) e OLHOFF et al. (1993) usaram essa categoria de microestrutura para otimização topológica em seus 
trabalhos. A microestrutura composta por material sólido com vazio interno (célula 1 na Fig. 2.8) consiste numa célula unitária com um buraco retangular no seu interior (BENDSØE; KIKUCHI, 1988, SUZUKI; KIKUCHI, 1991), cujas dimensões são definidas pelas variáveis de projeto $a$ e $b$ e o ângulo $\theta$. Assim, em cada ponto do domínio $(\Omega)$ define-se um material composto gerado pela repetição periódica de uma microestrutura de dimensões $a, b$, e $\theta$ (ou $\gamma$ na célula 2) correspondente aquele ponto. Dessa forma variando-se os valores de $a, b$, e $\theta$ (ou $\gamma$ na célula 2) ao longo do domínio estendido fixo durante a otimização altera-se a distribuição de material nesse domínio, de maneira que ao final da otimização existirão pontos com ar ( $a=b=1$ ou $\gamma=1$ na célula 2 ), pontos com sólido ( $a=b=0$ ou $\gamma=0$ na célula 2 ) e alguns pontos com materiais intermediários. Nesse sentido o problema consiste em se otimizar a distribuição de material num domínio perfurado com infinitos micro-furos.

Como já mencionado o método da homogeneização é um método robusto capaz de descrever as propriedades efetivas de um material homogeneizado a partir da definição de uma célula unitária ortotrópica, mas isso tem um custo: a introdução de novas variáveis de projeto (as dimensões $a, b$, e $\theta$ na célula 1 ou $\gamma$ na célula 2 ) no problema de otimização topológica. De fato, o aumento de variáveis de projeto no problema torna o método de Otimização Topológica desvantajoso, devido à complexidade da implementação numérica e ao alto custo computacional. Diante disso, o método de densidades vem conquistando a preferência dos pesquisadores da área de Otimização Topológica por ser um modelo mais simples de implementar e por utilizar somente uma variável de projeto: a densidade relativa do material em cada ponto domínio estendido fixo de projeto. Além de permitir obter um resultado muito semelhante ao obtido usando o método da homogeneização. O método de densidades é detalhado na seqüência.

\subsection{Método de densidades}

O modelo de material adotado neste trabalho é do tipo SIMP (Simple Isotropic Material with Penalization). O SIMP ou método de densidades 
(BENDSØE, 1989; ZHOU; ROZVANY, 1991; MLEJNEK, 1992) consiste em uma equação matemática que define o valor da densidade (variável de projeto que varia de zero a um valor máximo) em cada ponto do domínio $(\Omega)$ em função da propriedade efetiva do material base usado no projeto, essencialmente simulando uma microestrutura. Esta equação é dada por:

$$
\mathbf{C}(x)=\rho(x) \mathbf{C}_{0}
$$

onde $\rho(x)$ é interpretada como uma função de distribuição (contínua) de densidades (variável de projeto), $0 \leq \rho(x) \leq 1$ e $x \in \Omega$. Desta forma, podemos dizer que a densidade do material em cada ponto do domínio $(\Omega$ ) pode variar de "zero" (não há presença de material) à "um” (total presença de material).

Comparando a relação linear entre as propriedades efetivas $\mathbf{C}(x)$ e as do material base $\mathbf{C}_{0}$, vemos que a relação (Eq. 2.4) é inatingível para o meio contínuo, ou seja, como o valor da propriedade efetiva do material é definido pela sua microestrutura, nem todos os valores neste intervalo serão atingíveis, constituindo um limite superior para as propriedades do meio (BENDSØE, 1995). Assim, em cada ponto do domínio, qualquer material com propriedades variando entre zero (ou um valor muito baixo, para evitar problemas numéricos) e o valor do material de base é permitido. Isto torna contínuo a parametrização das propriedades do material no domínio, diferente da natureza 0-1 do problema (Eq. 2.2).

Matematicamente, a ocorrência de valores intermediários para a variável de projeto $\rho(x)$ estabelece a relaxação do problema e permite obter um espaço de solução fechado, o que é importante para garantir a existência da solução (BENDSØE, 1995), embora implique em obter uma estrutura com região de propriedades intermediárias. Como já mencionado, do ponto de vista prático, isto não é interessante, pois dificulta a interpretação final da topologia e torna inviável a fabricação.

Então, para evitar a ocorrência do excesso de densidades intermediárias 
(também conhecida como "escalas de cinza"), penalizam-se os valores intermediários de $\rho(x)$ da seguinte maneira (BENDSØE, 1989; ZHOU; ROZVANY, 1991; MLEJNEK, 1992):

$$
\mathbf{C}(x)=\rho(x)^{p} \mathbf{C}_{0}
$$

onde $p$ é o fator de penalidade que reduz as densidades intermediárias no resultado final. $\mathrm{O}$ tensor $\mathbf{C}_{0}$ é isotrópico e depende do módulo de elasticidade do material base $\left(E_{0}\right)$ e da razão de Poisson $\left(v_{0}\right)$. No SIMP o módulo de elasticidade do material em cada ponto do domínio ( $\Omega$ ) varia com a densidade $\rho$, enquanto que $v_{0}$ não depende de $\rho$.

O fator $p$ pode ser ajustado para evitar o aparecimento das "escalas de cinza”. Uma discussão interessante sobre este ajuste é feita em Bendsøe; Sigmund (1999), onde demonstraram que para garantir a existência de um modelo de material formado a partir da mistura de um material base e ar (buraco), sendo $E_{0} \gg E_{a r}$, no estado plano de tensão, as seguintes relações devem ser satisfeitas:

$$
\begin{aligned}
& \rho^{p} E_{0} \leq \frac{\rho E_{0}}{3-2 \rho} \quad \text { para } 0 \leq \rho \leq 1 \\
& p \geq \max \left\{\frac{2}{1-v_{0}}, \frac{4}{1+v_{0}}\right\}
\end{aligned}
$$

As Eq. (2.6) e (2.7) são baseadas nos limites de Hashin-Shtrikman (HASHIN; SHTRIKMAN, 1963 apud BENDSØE; SIGMUND, 1999), os quais definem uma região, onde as propriedades de microestruturas formadas a partir da mistura de dois materiais são fisicamente possíveis de serem obtidas. A partir das Eq. (2.6) e (2.7) podemos concluir que ambas são satisfeitas se $p \geq 3$. Embora 
matematicamente essa conclusão seja factível, na prática devemos tomar cuidado quanto a excessiva penalidade das densidades intermediárias (fator $p$ muito alto), pois a medida que aumentamos o fator $p$, aproximamos cada vez mais o problema contínuo num problema discreto, conforme observado em Rietz (2001), retornando ao problema da existência de solução na OT, devido as instabilidades numéricas geradas pela variação brusca da variação de projeto no problema discreto

Também é interessante notar que apesar da razão de Poisson $\left(v_{0}\right)$ não depender de $\rho(x)$, o valor de $p$ está implicitamente relacionada a ela pelas condições de Hashin-Shtrikman. Assim, a partir da Eq. (2.7) é simples verificar que, para os casos bidimensionais (2D), se $v_{0}$ for igual à $1 / 3$ (razão de Poisson do aço) o fator $p$ deverá ser igual a 3. A Fig. 2.9 mostra a comparação entre a curva limite de HashinShtrikman e as curvas da relação entre o módulo de elasticidade e a densidade do modelo SIMP para diversos valores de $p$.

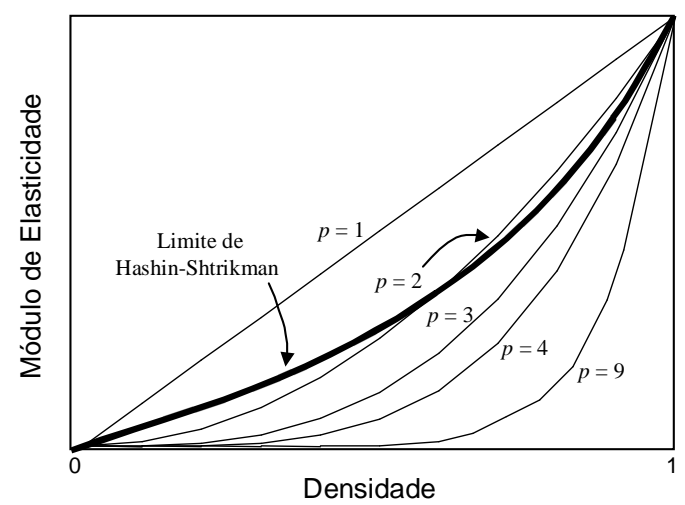

Figura 2.9 - Curva limite de Hashin-Shtrikman e curvas do modelo SIMP.

Já a utilização da Eq. (2.5) no método de densidades, para os problemas de OT, é uma alternativa menos complexa que também permite obter topologias bem definidas, isentas ou com uma quantidade mínima de "escalas de cinza". Bendsøe (1995) descreveu a prova da existência dessa solução. Mas, é importante deixar claro que as topologias obtidas são soluções ótimas locais, que buscam atender o ponto de 
vista de engenharia. Assim, devemos ter uma solução de compromisso, ou seja, obter uma solução ótima local, bem definida, o mais próxima possível da solução ótima global e que atenda os requisitos de viabilidade de construção para a estrutura projetada. O método de densidades é um modelo de material que, comparado ao método da homogeneização, é menos complexo de ser implementado numericamente num algoritmo de otimização. Há vários artigos científicos que implementam o MOT utilizando esse modelo de material (SIGMUND, 1996 e 1999; JONSMANN; SIGMUND; BOUWSTRA, 1999; YIN; YANG, 2000; PEDERSEN; BUHL; SIGMUND, 2001; LIMA; SILVA, 2002; e outros).

\subsection{Instabilidade xadrez}

Na maioria dos trabalhos sobre a aplicação do MOT observa-se uma certa complexidade para a eliminação de um aspecto comum a todos esses trabalhos: a formação de regiões com elementos de cor escura (presença de material) e elementos de cor branca (ausência de material), dispostos em forma de tabuleiro nos resultados obtidos. A Fig. 2.10 ilustra o exemplo de um resultado de OT onde surge a "instabilidade xadrez" (ou "checkerboard" como é conhecido na literatura internacional).

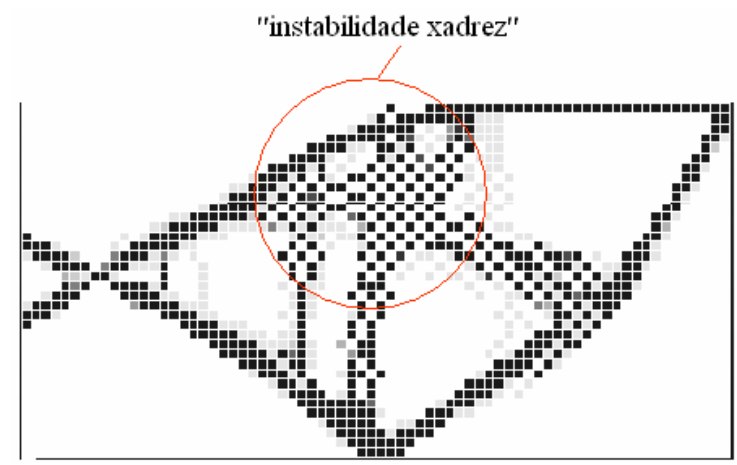

Figura 2.10 - "Instabilidade xadrez". 
A “instabilidade xadrez" é indesejável na solução do problema, pois não se configura numa ótima distribuição de material e sim num fenômeno que aparece devido à formulação (funções de interpolação) do elemento finito utilizado no processo de otimização. Kikuchi; Hollister; Yoo (1997) investigaram o comportamento local do arranjo de elementos finitos dispostos em forma de “instabilidade xadrez". Segundo eles, a ocorrência desse fenômeno nos resultados dos problemas de OT é que as aproximações numéricas introduzidas pelo método de elementos finitos (MEF) fazem com que o arranjo do material em forma de “instabilidade xadrez" seja mais rígido ao cisalhamento do que o arranjo uniforme (Fig. 2.11), considerando o mesmo volume de material em ambos os arranjos.

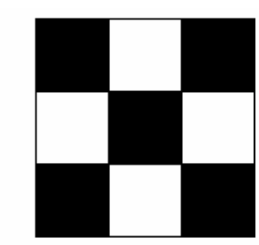

a) configur ação da "instabilidade xadrez"

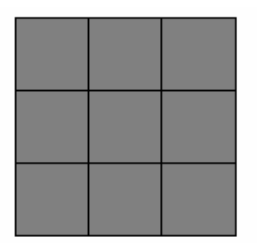

b) distribuição uniforme de material

Figura 2.11 - Arranjos da "instabilidade xadrez" e de material distribuído uniformemente.

A formação da "instabilidade xadrez" não é um aspecto exclusivo da Otimização Topológica, este fenômeno também se manifesta na solução de MEF para problemas com variacional misto. Um exemplo disto pode ser visto numa distribuição de pressões obtidas através da análise de MEF do problema de escoamento de fluidos de Stokes (ODEN; KIKUCHI; SONG, 1982), cujo variacional envolve velocidades e pressões. Estudos realizados por Díaz e Sigmund (DÍAZ; SIGMUND, 1995) e Jog e Haber (JOG; HABER, 1994, 1996), sugeriram que se o problema de OT for interpretado como um problema que contém um variacional misto, que envolve o campo de densidades (variáveis) e o campo de deslocamentos, a formação da "instabilidade xadrez" pode ser evitada usando diferentes funções de 
interpolação (de mesma ordem) para ambos os campos (densidades e deslocamentos). Estas conclusões se assemelham às obtidas no problema de Stokes, onde determinadas combinações de interpolação para o campo de deslocamentos e para o campo de pressões se mostra instável. O trabalho de Jog; Haber $(1994,1996)$, propõe uma série de testes (baseados em observações práticas ou no estudo matemático do problema variacional misto) para avaliar se uma determinada combinação de interpolações de densidades e deslocamentos resulta em configurações instáveis ou estáveis.

Vários trabalhos na literatura mencionam a formação da "instabilidade xadrez" em seus resultados de OT, mas poucos discutem profundamente o assunto, tanto que a sua solução ainda é um tema bastante polêmico e pouco explorado. Em suma, a maioria desses trabalhos, sugerem duas formas distintas para eliminação da formação da "instabilidade xadrez" nos problemas de OT. Uma delas é aumentar a ordem do elemento finito e a outra é utilizar métodos de filtragem ou de controle de gradiente.

Como visto anteriormente, para uma mesma quantidade de material o arranjo de "instabilidade xadrez" (Fig. 2.11a), com elementos de baixa ordem (4 nós), produz uma configuração mais rígida em termos de deformação de cisalhamento do que o arranjo uniforme de material. Aumentar a ordem do elemento significa aumentar o número de nós do elemento finito. Elementos de alta ordem possuem funções de interpolação que representam melhor o campo de deslocamentos no elemento. Então, a utilização de elementos de alta ordem permitem reduzir o erro induzido aos termos de deformação de cisalhamento no elemento. Isso provavelmente explica a razão pela qual a "instabilidade xadrez" não aparece quando se utiliza elementos finitos de alta ordem nos problemas de Otimização Topológica. Sigmund; Petersson; (1998b) utilizaram essa solução para obter resultados isentos da formação da "instabilidade xadrez" para o problema de OT cuja função objetivo considera máxima rigidez de uma estrutura com restrição de volume de material. Mas, eles concluíram que esta é uma alternativa cara devido ao alto custo computacional. Além disso, Díaz; Sigmund (1995) demonstraram que, para determinados valores do fator de penalidade $p$ dos problemas de OT que utilizam o método de densidades (veja item 2.6 deste capítulo), elementos de 9 nós também 
podem causar a formação da "instabilidade xadrez". Segundo eles, não é interessante a utilização desses elementos para a seguinte relação:

$$
p>\frac{\log \left(2\left(6-5 v_{0}\right)\right)}{\log 2}
$$

onde $v_{0}$ é a razão de Poisson.

A maioria dos trabalhos que envolvem o MOT, inclusive este, usa o elemento quadrilátero de 4 nós, por ele ter uma formulação simples de ser implementada num software e além disso a matriz de rigidez global da estrutura é menor em relação a alguma outra que considera elementos de ordem maior, o que implica em economia de tempo computacional, se considerarmos a natureza iterativa do MOT. Desta forma, uma outra alternativa muito usada para a eliminação da "instabilidade xadrez" nos problemas de OT é a introdução de métodos de controle das variáveis de projeto (densidades). Variações bruscas nos gradientes das variáveis de projeto favorecem a formação da "instabilidade xadrez", sendo assim a utilização de um método de controle sobre a variação espacial das variáveis de projeto evita esse fenômeno, além de permitir um razoável controle da complexidade da topologia (BOURDIN, 2001) obtida pelo método de OT. A suavização da variação das variáveis de projeto nos problemas de OT é feita, ou através de restrições inseridas na própria formulação do problema de otimização, como em Haber; Jog; Bendsøe (1996) que acrescentam uma restrição de perímetro na formulação, ou Cardoso; Fonseca (1999) que impõe uma restrição aos gradientes das variáveis de projeto no problema de minimização de volume com restrição de flexibilidade. Swan; Kosaka (1997) propuseram um filtro espacial de vizinhança fixa, no qual a densidade de cada elemento depende das densidades dos elementos vizinhos. Apesar deste filtro ser eficaz para evitar a formação da "instabilidade xadrez" ele é dependente do tamanho da malha de elementos finitos, ou seja, obtêm-se resultados de OT diferentes conforme aumentamos a discretização do domínio estendido fixo de projeto. Cardoso; Fonseca (1999) propuseram uma versão baseada no filtro de Swan; Kosaka (1997) na qual implementam um conceito de raio de abrangência no filtro para torná- 
lo independente ao refino da malha de elementos finitos. Portanto, o filtro desenvolvido por Cardoso; Fonseca (1999) consiste em uma regularização que atua nas restrições do problema de otimização.

No entanto, essa regularização também pode ser implementada adicionandose um termo extra na função objetivo que ao ser minimizado limita a variação brusca dos gradientes de densidade no domínio. Essa abordagem foi adotada por Pereira (2001) em que o termo do tipo:

$$
\frac{1}{2} r_{\rho} \int_{\Omega}(\nabla \rho)^{t}(\nabla \rho) d \Omega
$$

é adicionado na função objetivo para atuar sobre as variações de densidades no domínio. Essa abordagem também foi bem sucedida e se mostrou eficiente.

Neste trabalho é utilizado o filtro espacial proposto por Cardoso; Fonseca (1999), cuja introdução teórica e implementação numérica serão vistos mais adiante. 


\section{Capítulo 3: Piezeletricidade e o MEF piezelétrico}

\subsection{Piezeletricidade}

A piezeletricidade é uma propriedade que certos materiais apresentam, que pode ser definida como a polarização elétrica produzida por uma deformação mecânica em certos tipos de cristais. Quando um material piezelétrico é submetido a um campo elétrico, sofre alteração em suas dimensões. O inverso do efeito piezelétrico também é válido, ou seja, quando o material piezelétrico sofre uma deformação, um campo elétrico é gerado.

Os efeitos piezelétricos são características de todos os cristais ferroelétricos. A denominação "ferroeletricidade" é histórica e é devido à similaridade do fenômeno ferroelétrico com o ferromagnetismo. A similaridade é principalmente fenomenológica, ou seja, exatamente como os materiais ferromagnéticos exibem uma magnetização espontânea e o efeito de histerese na relação entre a magnetização e o campo magnético, o cristal ferroelétrico também apresenta uma espontânea polarização elétrica e o efeito de histerese entre o deslocamento dielétrico e o campo elétrico. Este comportamento é principalmente observado em temperaturas abaixo da temperatura de transição ou de Curie. Para pontos acima desta temperatura, o material perde as propriedades piezelétricas e mostra um comportamento dielétrico normal. Para entender melhor a piezeletricidade, nos itens seguintes serão abordados os materiais piezelétricos (item 3.2) e as equações do meio piezelétrico (item 3.3).

No item 3.4 é feita uma descrição sobre o método dos elementos finitos (MEF) aplicado à piezeletricidade e o detalhamento do elemento não-piezelétrico utilizado no processo de otimização topológica. 


\subsection{Material piezelétrico}

Alguns dos materiais ferroelétricos são: o titanato de bário (BaTiO3), o niobato de lítio, e o titanato zircanato de chumbo (cerâmicas PZT). Os cristais PZT (usados neste trabalho), são cúbico centro-simétricos (isotrópicos) antes da polarização e após a polarização são da classe de simetria hexagonal da família $6 \mathrm{~mm}$ abaixo da temperatura de Curie, como mostrado na figura abaixo (Fig. 3.1).
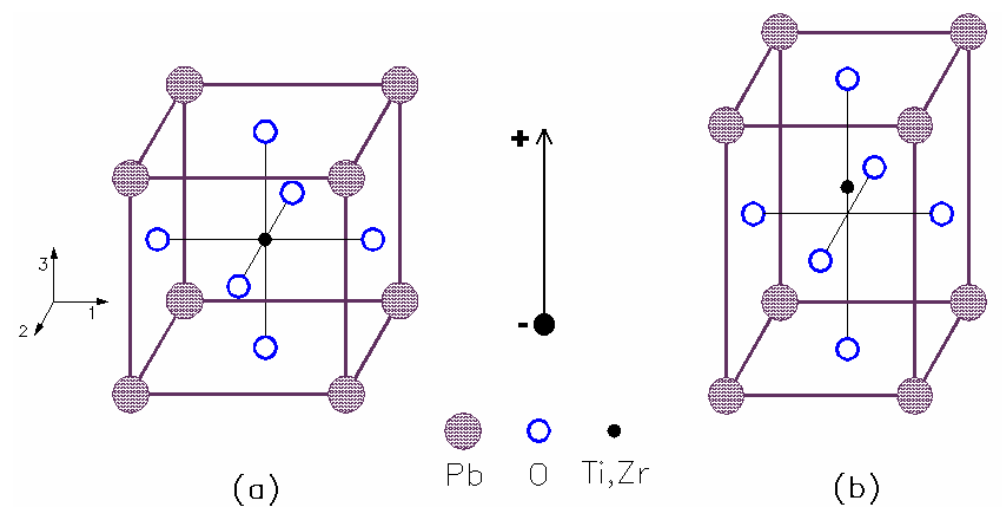

(b)

Figura 3.1- Cristalografia do cristal PZT: (a) não-polarizado e (b) polarizado.

A separação entre os íons positivos e negativos é a causa do comportamento do dipolo elétrico (ver Fig. 3.1 (b)). Grupos de dipolos com orientação paralela são chamadas de domínios de Weiss. Os domínios de Weiss são aleatórios no estado natural do material PZT antes do tratamento de polarização ter sido finalizado. Por isto, propositadamente um campo elétrico $(>2000 \mathrm{~V} / \mathrm{mm})$ é aplicado na cerâmica piezelétrica. Com a aplicação do campo, o material expande na direção axial ao campo e contraí na direção perpendicular ao campo. Os dipolos elétricos alinham-se e bruscamente suspendem o alinhamento e arranjam-se nesta direção. Ou seja, quando uma voltagem elétrica é aplicada no material piezelétrico, o domínio de Weiss aumenta o alinhamento proporcionalmente à voltagem. O resultado é a mudança das dimensões (expansão e contração) do material PZT. 


\subsection{Equações constitutivas piezelétricas na forma tensorial e matricial}

A piezeletricidade é um acoplamento bilinear entre variáveis mecânicas e elétricas, ver Fig. 3.2. No entanto, para alguns materiais específicos e determinado campo elétrico, o fenômeno da eletroestricção aparece combinado com a piezeletricidade. A eletroestricção é um efeito não-linear que está presente em todos os materiais dielétricos. Surge, devido a acoplamentos eletromecânicos de alta ordem, por exemplo, quando o material piezelétrico é empregado em estruturas ressonantes, tipo sonares (IEEE, 1978 apud IKEDA, 1996). Mas, para os materiais e magnitude do campo elétrico considerado neste trabalho, pode-se considerar a piezeletricidade como o fator dominante do acoplamento eletromecânico.

O efeito piezelétrico direto é quando a polarização elétrica é produzida por tensões mecânicas. A relação inversa do efeito piezelétrico direto também é válida, ou seja, o cristal se deforma quando é sujeito a um campo elétrico. Ambos efeitos são propriedades fundamentais idênticas manifestadas nos cristais.

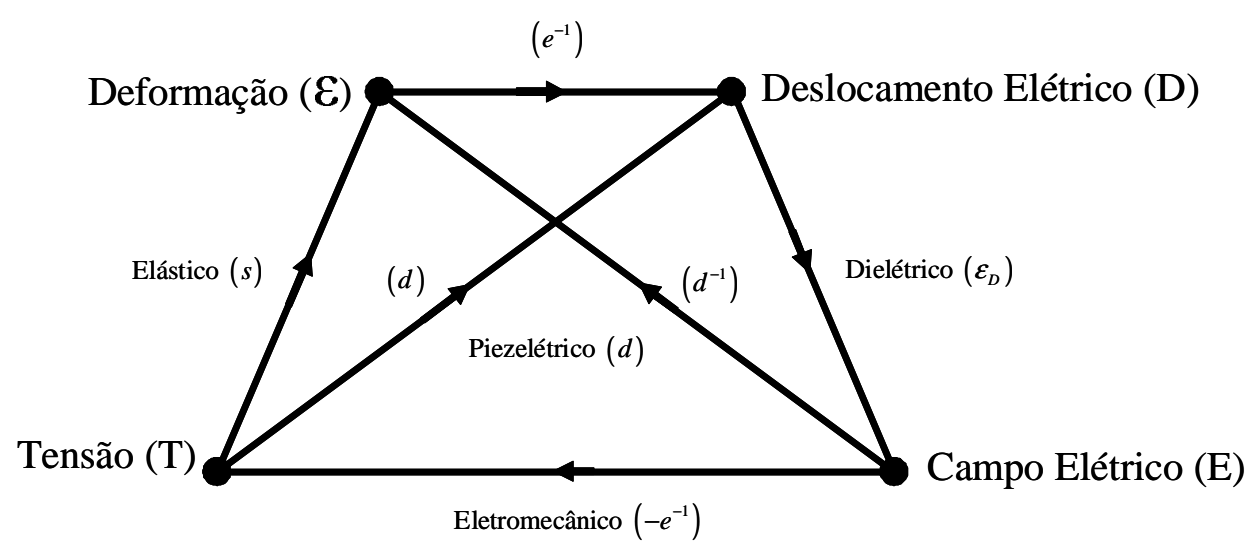

Figura 3.2- Diagrama entre as interações mecânicas e elétricas.

As grandezas representadas nos vértices inferiores do trapézio (Fig. 3.2) são 
variáveis intensivas e aquelas representadas nos vértices superiores são variáveis extensivas, ou seja, as variáveis intensivas são grandezas independentes e as variáveis extensivas são grandezas dependentes. As linhas que conectam os vértices indicam o acoplamento entre as respectivas variáveis, mas na Fig. 3.2 está indicado apenas o efeito direto entre elas.

As relações constitutivas piezelétricas relacionam variáveis elétricas e mecânicas. As variáveis mecânicas são geralmente a deformação $\mathcal{E}$ e a tensão $\boldsymbol{T}$ e as variáveis elétricas são o deslocamento elétrico $\boldsymbol{D}$ e o campo elétrico $\boldsymbol{E}$, sendo deduzidas a partir da função de Gibbs (potenciais termodinâmicos). Considerando o modelo unidimensional do sólido piezelétrico e tendo $\boldsymbol{E}$ e $\boldsymbol{E}$ das equações piezelétricas como variáveis independentes, tem-se:

$$
\mathbf{T}=\mathbf{T}(\mathcal{E}, \mathbf{E}) \text { e } \mathbf{D}=\mathbf{D}(\mathcal{E}, \mathbf{E}),
$$

e utilizando os primeiros termos da expansão de Taylor:

$$
\begin{aligned}
& \mathbf{T}=\left(\frac{\partial \mathbf{T}}{\partial \mathcal{E}}\right)_{E} \mathcal{E}+\left(\frac{\partial \mathbf{T}}{\partial \mathbf{E}}\right)_{S} \mathbf{E} \\
& \mathbf{D}=\left(\frac{\partial \mathbf{D}}{\partial \mathcal{E}}\right)_{E} \mathcal{E}+\left(\frac{\partial \mathbf{D}}{\partial \mathbf{E}}\right)_{S} \mathbf{E}
\end{aligned}
$$

onde os efeitos magnéticos e térmicos, e os termos não-lineares foram desprezados (RISTIK, 1983). Então podemos definir as seguintes constantes:

$$
\begin{aligned}
& \mathbf{c}^{E}=\left(\frac{\partial \mathbf{T}}{\partial \mathcal{E}}\right)_{E} ; \mathbf{e}^{E}=\left(\frac{\partial \mathbf{D}}{\partial \mathcal{E}}\right)_{E} \\
& \mathbf{e}^{S}=\left(\frac{\partial \mathbf{T}}{\partial \mathbf{E}}\right)_{S} ; \boldsymbol{\varepsilon}_{D}^{S}=\left(\frac{\partial \mathbf{D}}{\partial \mathbf{E}}\right)_{S}
\end{aligned}
$$


onde $\mathbf{c}^{E}$, e e $\boldsymbol{\varepsilon}_{D}^{S}$ são as constantes elásticas, piezelétricas e dielétricas, respectivamente, e os subscritos indicam que $\mathbf{c}^{E}$ e $\mathbf{e}^{E}$ devem ser obtidos sob um campo elétrico constante (por exemplo nulo) e $\mathbf{e}^{S}$ e $\boldsymbol{\varepsilon}_{D}^{S}$ sob deformação nula.

A medição das constantes de rigidez e dielétricas definidas acima depende das condições de contorno como indicado. Considere, um campo elétrico sendo aplicado no meio piezelétrico. Realizando uma medição da constante $\boldsymbol{\varepsilon}_{D}^{S}$, sem a cerâmica estar bloqueada mecanicamente, obtém-se um valor errado de $\boldsymbol{\varepsilon}_{D}^{S}$, pelo fato de uma deformação surgir em resposta da tensão, contribuindo na Eq. (3.2) para alterar a relação entre $\boldsymbol{D}$ e $\boldsymbol{E}$. O mesmo vale para as outras constantes. Assim a medição das propriedades elétricas do meio piezelétrico é dependente das restrições mecânicas, devido ao acoplamento piezelétrico. Entretanto seguindo considerações termodinâmicas, mostra-se que o efeito piezelétrico inverso $\mathbf{e}^{S}$ é uma conseqüência do efeito piezelétrico direto $\mathbf{e}^{E}$, assim:

$$
-\mathbf{e}^{S}=\mathbf{e}^{E}=\mathbf{e}
$$

e pode-se escrever as equações (3.2) como:

$$
\begin{aligned}
& \mathbf{T}=\mathbf{c}^{E} \mathcal{E}-\mathbf{e E} \\
& \mathbf{D}=\mathbf{e} \mathcal{E}+\boldsymbol{\varepsilon}_{D}^{S} \mathbf{E}
\end{aligned}
$$

Alterando-se em pares as variáveis independentes escolhidas, obtém-se outras expressões das equações piezelétricas (RISTIK, 1983; IKEDA, 1996):

$$
\begin{aligned}
& \mathcal{E}=\mathbf{s}^{E} \mathbf{T}-\mathbf{d E} \\
& \mathbf{D}=\mathbf{d} \mathbf{T}+\boldsymbol{\varepsilon}_{D}^{T} \mathbf{E}
\end{aligned}
$$




$$
\begin{aligned}
& \mathcal{E}=\mathbf{s}^{D} \mathbf{T}-\mathbf{g D} \\
& \mathbf{E}=-\mathbf{g} \mathbf{T}+\boldsymbol{\beta}^{T} \mathbf{D}
\end{aligned}
$$

$$
\begin{aligned}
& \mathbf{T}=\mathbf{c}^{D} \mathcal{E}-\mathbf{h D} \\
& \mathbf{E}=-\mathbf{h} \mathcal{E}+\boldsymbol{\beta}^{S} \mathbf{D}
\end{aligned}
$$

No conjunto de equações (Eq. (3.6) à (3.8)) os novos termos são as constantes piezelétricas: $\boldsymbol{h}$, campo elétrico/deformação; $\boldsymbol{d}$, deformação/campo elétrico e $\boldsymbol{g}$, campo elétrico/tensão mecânica; $\beta$ é a impermeabilidade dielétrica. Os subscritos designam: ${ }^{E}$, a campo elétrico constante; ${ }^{D}$, a deslocamento elétrico constante; ${ }^{T}$, a tensão mecânica constante $\mathrm{e}^{S}$, a deformação mecânica constante.

As equações anteriores que descrevem o meio piezelétrico podem ser apresentadas na sua forma tensorial e matricial (RISTIK, 1983). Na forma tensorial as equações piezelétricas são representadas na seguinte maneira:

$$
\begin{aligned}
& T_{i j}=c_{i j k l}^{E} \mathcal{E}_{k l}-e_{k i j} E_{k} \\
& D_{i}=e_{i j k} \mathcal{E}_{j k}+\mathcal{E}_{D_{j k}}^{S} E_{m}
\end{aligned}
$$

sendo: $\mathbf{E}=-\nabla \phi$

e considerando a Eq. (3.10), pode-se escrever a Eq. (3.9), como:

$$
\begin{aligned}
& T_{i j}=c_{i j k l}^{E} \mathcal{E}_{k l}+e_{k i j}\left(-E_{k}\right) \\
& D_{i}=e_{i k l} \mathcal{E}_{k l}+\left(-\mathcal{E}_{D_{i k}}^{S}\right)\left(-E_{k}\right)
\end{aligned}
$$

onde $c_{i j k l}^{E}, e_{i j}^{S}, \varepsilon_{D_{i k}}^{S}, T_{i j}$ e $\mathcal{E}_{j k}$ são os tensores de rigidez elástica, piezelétrico, dielétricos, de tensão e de deformação respectivamente. 
onde:

$$
\mathcal{E}_{j k}=\frac{1}{2}\left(\frac{\partial u_{k}}{\partial x_{l}}+\frac{\partial u_{l}}{\partial x_{j}}\right)
$$

Os vetores campo elétrico $(\mathbf{E})$ e do deslocamento elétrico $(\mathbf{D})$ são dados por:

$$
\mathbf{E}=\left\{\begin{array}{l}
E_{x} \\
E_{y} \\
E_{z}
\end{array}\right\} \text { e } \mathbf{D}=\left\{\begin{array}{l}
D_{x} \\
D_{y} \\
D_{z}
\end{array}\right\}
$$

Assumindo de uma forma geral, como direção da polarização a direção 3, independente desta ser a direção $y$ ou $z$.

A Eq. (3.9) pode ser escrita na forma matricial, como:

$$
\left\{\begin{array}{l}
\mathbf{T} \\
\mathbf{D}
\end{array}\right\}=\left[\begin{array}{cc}
\mathbf{c}^{E} & \mathbf{e}^{t} \\
\mathbf{e} & \boldsymbol{\varepsilon}_{D}^{S}
\end{array}\right]\left\{\begin{array}{l}
\boldsymbol{E} \\
\mathbf{E}
\end{array}\right\}
$$

De forma análoga:

$$
\left\{\begin{array}{l}
\mathcal{E} \\
\mathbf{D}
\end{array}\right\}=\left[\begin{array}{ll}
\mathbf{s}^{E} & \mathbf{d}^{t} \\
\mathbf{d} & \boldsymbol{\varepsilon}_{D}^{T}
\end{array}\right]\left\{\begin{array}{l}
\mathbf{T} \\
\mathbf{E}
\end{array}\right\}
$$

onde o índice " $\mathrm{t}$ " indica transposta da matriz.

As matrizes das equações (3.14) e (3.15), podem ser definidas como um 
matriz elasto-piezelétrica-dielétrica (Eq. (3.16)) (RISTIK, 1983), sendo: a matriz elástica de dimensão 6 × 6 , com 9 constantes elásticas; a matriz piezelétrica de dimensão 6 × 3 e $3 \times 6$, com 5 constantes piezelétricas e a matriz dielétrica de dimensão $3 \mathbf{x} 3$, com 3 constantes dielétricas, como mostrados abaixo:

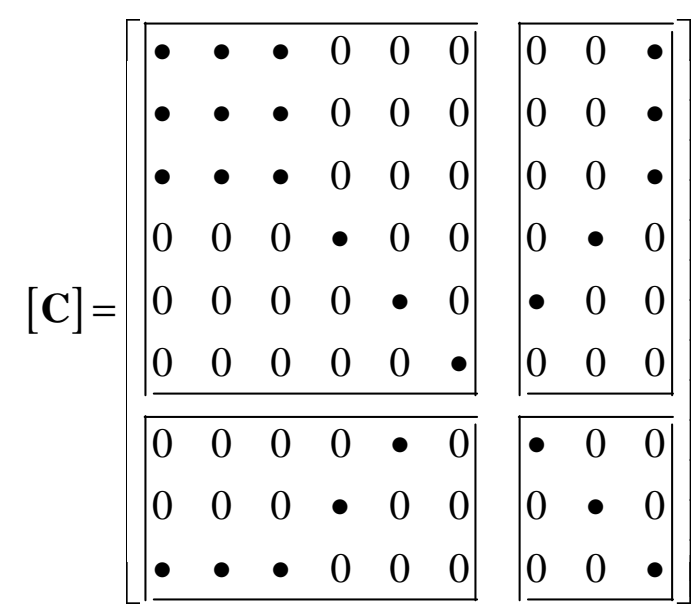

Os materiais piezelétricos são elasticamente e piezeletricamente anisotrópicos na direção 3 e isotrópicos no plano 12 (AULD, 1990). Para os materiais das classes hexagonais (KINO, 1987; AULD, 1990 e IKEDA, 1996) com anisotropia na direção 3 são mostrados a seguir o tensor elástico $c_{i j k l}$, o tensor dielétrico $\varepsilon_{D_{i j}}$ e o tensor piezelétrico $e_{i j k}$ na forma matricial.

A matriz de constante de rigidez elástica c é ortotrópico para o material piezelétrico sendo dada pela teoria da elasticidade anisotrópica por:

$$
[\mathbf{c}]=\left[\begin{array}{cccccc}
c_{11} & c_{12} & c_{13} & 0 & 0 & 0 \\
c_{12} & c_{22} & c_{13} & 0 & 0 & 0 \\
c_{13} & c_{13} & c_{33} & 0 & 0 & 0 \\
0 & 0 & 0 & c_{44} & 0 & 0 \\
0 & 0 & 0 & 0 & c_{55} & 0 \\
0 & 0 & 0 & 0 & 0 & c_{66}
\end{array}\right]
$$


onde: $c_{66}=\frac{1}{2}\left(c_{11}-c_{12}\right)$

As componentes do tensor dielétrico $\boldsymbol{\varepsilon}_{D}$ são:

$\left[\varepsilon_{D}\right]=\left[\begin{array}{ccc}\varepsilon_{D_{11}} & 0 & 0 \\ 0 & \varepsilon_{D_{11}} & 0 \\ 0 & 0 & \varepsilon_{D_{33}}\end{array}\right]$

As constantes piezelétricas de tensão mecânica $\boldsymbol{e}$ são:

$$
[e]=\left[\begin{array}{cccccc}
0 & 0 & 0 & 0 & e_{15} & 0 \\
0 & 0 & 0 & e_{15} & 0 & 0 \\
e_{31} & e_{31} & e_{33} & 0 & 0 & 0
\end{array}\right]
$$

Assim, podemos escrever a equação (3.14) como:

$$
\left\{\begin{array}{l}
T_{11} \\
T_{22} \\
T_{33} \\
T_{23} \\
T_{13} \\
T_{12} \\
D_{1} \\
D_{2} \\
D_{3}
\end{array}\right\}=\left[\begin{array}{ccccccccc}
c_{11}^{E} & c_{12}^{E} & c_{13}^{E} & 0 & 0 & 0 & 0 & 0 & e_{31} \\
c_{12}^{E} & c_{11}^{E} & c_{13}^{E} & 0 & 0 & 0 & 0 & 0 & e_{31} \\
c_{13}^{E} & c_{13}^{E} & c_{33}^{E} & 0 & 0 & 0 & 0 & 0 & e_{33} \\
0 & 0 & 0 & c_{44}^{E} & 0 & 0 & 0 & e_{15} & 0 \\
0 & 0 & 0 & 0 & c_{44}^{E} & 0 & e_{15} & 0 & 0 \\
0 & 0 & 0 & 0 & 0 & c_{66}^{E} & 0 & 0 & 0 \\
0 & 0 & 0 & 0 & e_{15} & 0 & -\varepsilon_{D_{11}} & 0 & 0 \\
0 & 0 & 0 & e_{15} & 0 & 0 & 0 & -\mathcal{E}_{D_{11}} & 0 \\
e_{31} & e_{31} & e_{33} & 0 & 0 & 0 & 0 & 0 & -\mathcal{E}_{D_{33}}
\end{array}\right]\left\{\begin{array}{l}
\mathcal{E}_{11} \\
\mathcal{E}_{22} \\
\mathcal{E}_{33} \\
\mathcal{E}_{23} \\
\mathcal{E}_{13} \\
\mathcal{E}_{12} \\
-E_{1} \\
-E_{2} \\
-E_{3}
\end{array}\right\}
$$

Os valores das constantes elásticas, piezelétricas e dielétricas são obtidos de trabalhos, livros e artigos (Nader, 2002). 
O programa de elementos finitos é desenvolvido para modelos bidimensionais considerando o estado plano (tensão ou deformação). Muitos problemas de elasticidade podem ser tratados satisfatoriamente em duas dimensões, pela teoria da elasticidade plana. Nos modelos bidimensionais, o programa considera a polarização na direção 2 , enquanto que nos modelos tridimensionais a polarização pode ser considerada na direção 3. A equação constitutiva (Eq. (3.20)) segue a definição das direções do IEEE (IEEE, 1996), no qual a polarização está na direção 3. Dessa forma, para realizar uma simulação bidimensional há necessidade de mudança de base da matriz das equações constitutivas (Eq. (3.20)).

Os problemas envolvidos nas análises de estados planos podem ser definidos por um determinado número de restrições e suposições nos estados planos de tensão e deformação. A solução pode ser obtida através das considerações: de estado plano de tensão mecânica ou do estado plano de deformações mecânicas. Esses estados planos são descritos em termos das hipótesis físicas da estrutura analisada, como mostrado nas Fig 3.3.

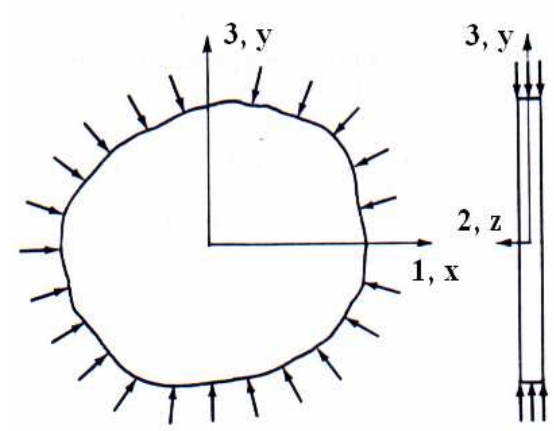

(a)

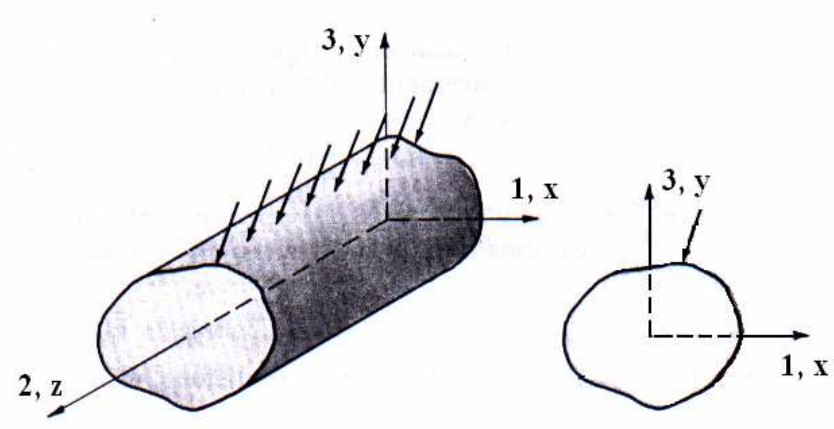

(b)

Figura 3.3. (a) estado plano de tensões mecânicas (EPTM); (b) estado plano de deformações mecânicas (EPDM). A polarização (P) de um material piezelétrico é considerada na direção 3 . 


\subsubsection{Estado Plano de Tensões Mecânicas (EPTM)}

Esta consideração é utilizada em corpos que possuem uma geometria com a largura (direção 1) e o comprimento (direção 3) em dimensões comparáveis e no entanto muito maiores que a espessura (direção 2), como mostra a Fig. 3.3(a). As cargas mecânicas são aplicadas uniformemente sobre a espessura da placa. Portanto, o estado plano de tensões mecânicas da Fig. 3.3(a) tem como hipóteses:

$$
T_{z z}=T_{x z}=T_{y z}=0
$$

dessa forma, a matriz da lei de Hooke para um material isotrópico torna-se:

$$
\left\{\begin{array}{l}
T_{x x} \\
T_{y y} \\
T_{x y}
\end{array}\right\}=\frac{E}{1-v^{2}}\left[\begin{array}{ccc}
1 & v & 0 \\
v & 1 & 0 \\
0 & 0 & \frac{1-v}{2}
\end{array}\right]\left\{\begin{array}{l}
\mathcal{E}_{x x} \\
\mathcal{E}_{y y} \\
\mathcal{E}_{x y}
\end{array}\right\}
$$

No caso dos materiais piezelétricos da classe de simetria hexagonal da família $6 m m$ o estado plano de tensões mecânicas assume como condição extra que a componente do deslocamento elétrico na direção 3 é nula:

$$
D_{z}=0
$$

Após as modificações na matriz das equações constitutivas piezelétrica (3.20), esta assume a seguinte forma: 


$$
\left\{\begin{array}{l}
T_{x x} \\
T_{y y} \\
T_{x y} \\
D_{x} \\
D_{y}
\end{array}\right\}=\left[\begin{array}{ccccc}
c_{11}^{E}-\frac{c_{12}^{E 2}}{c_{11}^{E}} & c_{13}^{E}-\frac{c_{12}^{E} c_{31}^{E}}{c_{11}^{E}} & 0 & 0 & e_{31}-\frac{e_{31} c_{12}^{E}}{c_{11}^{E}} \\
c_{13}^{E}-\frac{c_{12}^{E} c_{13}^{E}}{c_{11}^{E}} & c_{33}^{E}-\frac{c_{31}^{E 2}}{c_{11}^{E}} & 0 & 0 & e_{33}-\frac{e_{31} c_{13}^{E}}{c_{11}^{E}} \\
0 & 0 & c_{44}^{E} & e_{15} & 0 \\
0 & 0 & e_{15} & -\varepsilon_{D_{11}}^{S} & 0 \\
e_{31}-\frac{e_{31} c_{12}^{E}}{c_{11}^{E}} & e_{33}-\frac{e_{31} c_{13}^{E}}{c_{11}^{E}} & 0 & 0 & -\left(-\mathcal{E}_{D_{11}}^{S}+\frac{e_{31}^{2}}{c_{11}^{E}}\right.
\end{array}\right]\left\{\begin{array}{l}
\mathcal{E}_{x x} \\
\mathcal{E}_{y y} \\
\mathcal{E}_{x y} \\
-E_{x} \\
-E_{y}
\end{array}\right\}(3.22)
$$

Portanto, no caso do estado plano de tensões há contribuição dos termos da rigidez na direção 2, pois nessas considerações assume-se que a estrutura analisada possui dimensões finitas e a espessura (direção 2) influencia no comportamento da estrutura. Os termos piezelétricos sofrem influência das constantes elásticas e os termos dielétricos sofrem influência das constantes elásticas e piezelétricas.

\subsection{2- Estado Plano de Deformações Mecânicas (EPDM)}

Esta consideração é utilizada em corpos que possuem a espessura (direção 2) em dimensões muito superiores à largura (direção 1) e ao comprimento (direção 3), como mostra a Fig. 3.3(b). As cargas mecânicas são distribuídas uniformemente em relação à espessura e atuam perpendiculares a esta. Portanto, o estado plano de deformações mecânicas (Fig. 3.3(b)) assume como hipótese que a componente do deslocamento na direção 3 é nula:

$$
u_{z}=0
$$

Dessa forma, as deformações ficam: 
$\boldsymbol{\mathcal { E }}_{z z}=0 ; \boldsymbol{\mathcal { E }}_{y z}=0$ e $\boldsymbol{\mathcal { E }}_{x z}=0$

Dessa forma a matriz da lei de Hooke para o material isotrópico torna-se:

$$
\left\{\begin{array}{l}
T_{x x} \\
T_{y y} \\
T_{x y}
\end{array}\right\}=\frac{E}{(1+v)(1-2 v)}\left[\begin{array}{ccc}
1-v & v & 0 \\
v & 1-v & 0 \\
0 & 0 & \frac{1-2 v}{2}
\end{array}\right]\left\{\begin{array}{l}
\mathcal{E}_{x x} \\
\mathcal{E}_{y y} \\
\mathcal{E}_{x y}
\end{array}\right\}
$$

No caso da simulação de um material piezelétrico em estado plano de deformações mecânicas, assume-se também que a componente do campo elétrico da direção 3 é nula:

$$
E_{z}=0
$$

Dessa forma, a matriz que representa as equações constitutivas piezelétricas se altera para a forma:

$$
\left\{\begin{array}{l}
T_{x x} \\
T_{y y} \\
T_{x y} \\
D_{x} \\
D_{y}
\end{array}\right\}=\left[\begin{array}{ccccc}
c_{11}^{E} & c_{13}^{E} & 0 & 0 & e_{31} \\
c_{13}^{E} & c_{33}^{E} & 0 & 0 & e_{33} \\
0 & 0 & c_{44}^{E} & e_{15} & 0 \\
0 & 0 & e_{15} & -\varepsilon_{D_{11}}^{S} & 0 \\
e_{31} & e_{33} & 0 & 0 & -\varepsilon_{D_{33}}^{S}
\end{array}\right]\left\{\begin{array}{l}
\mathcal{E}_{x x} \\
\mathcal{E}_{y y} \\
\mathcal{E}_{x y} \\
-E_{x} \\
-E_{y}
\end{array}\right\}
$$

Portanto, no estado plano de deformações, não há contribuição dos termos da direção 2, pois assume-se que a estrutura analisada é infinita na direção da espessura, ou que está rigidamente fixada nessa direção. Os termos piezelétricos não sofrem influência das constantes elásticas e os termos dielétricos não sofrem 
influência dos termos elásticos e piezelétricos.

As relações constitutivas definidas anteriormente (Eq. (3.21) à (3.24) ), foram simplificadas para serem aplicadas no MEF que será desenvolvido no item seguinte.

\subsection{Implementação do MEF piezelétrico}

O problema de otimização, apresentado no capítulo 4, pode ser resolvido numericamente através de um algoritmo computacional que combina o método de otimização de programação linear seqüencial (PLS) (DANTZIG, 1963; YANG; CHUANG, 1994 e HAFTKA; GURDAL; KAMAT, 1996) com o método numérico de análise por elementos finitos (BATHE, 1996).

O MEF desenvolvido neste software é constituído de duas formulações de elemento distintas. Um elemento piezelétrico que possui três graus de liberdade por nó (deslocamentos mecânicos no plano e o terceiro o deslocamento elétrico) e que não é considerado na otimização (elemento não otimizável). Um elemento nãopiezelétrico que possui dois graus de liberdade por nó (deslocamentos mecânicos no plano), e que discretiza a região otimizável.

A otimização topológica gera resultados com topologias complexas, assim, necessitamos de um método robusto, confiável e genérico como o Método de Elementos Finitos para análise estrutural. Portanto, nesta seção descreveremos brevemente a formulação do método aplicado ao projeto de atuadores piezelétricos flextensionais. A formulação do MEF é esquematizado como na figura abaixo (Fig 3.4): 


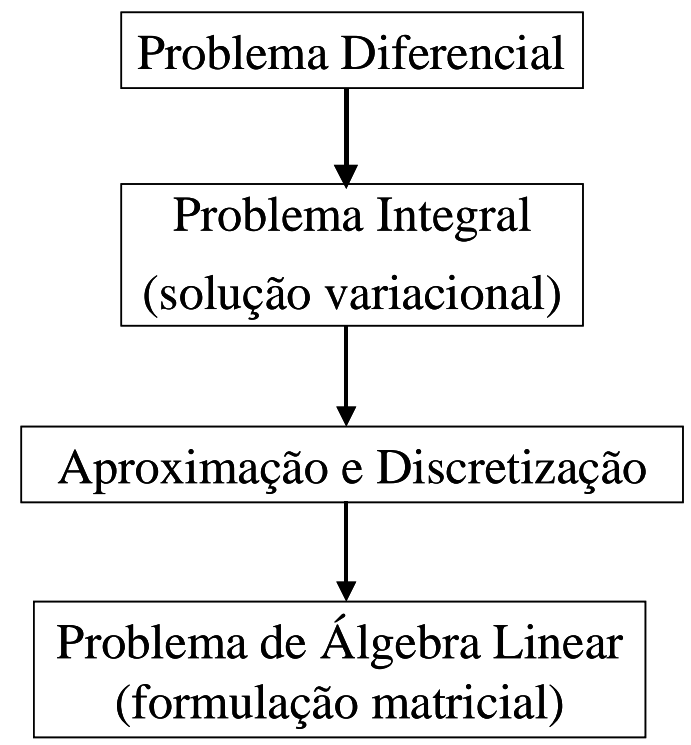

Figura 3.4- Apresentação esquemática do MEF.

Para se iniciar a análise de elementos finitos como descrito na figura acima (Fig. 3.4), é necessário discretizar o modelo a ser projetado em uma malha de elementos finitos que represente, através de matrizes numéricas, o domínio de projeto (geometria), as condições de contorno de projeto (restrições de deslocamentos e cargas elétricas, e carregamentos tanto elétricos quanto mecânicos aplicados) e do princípio variacional, que estabelece em cada instante o equilíbrio da energia cinética e potencial em todos os pontos do domínio considerado. Consiste num método de transformação de um problema apresentado na forma integral, em um problema aproximado de álgebra linear, onde os coeficientes são integrais avaliadas sobre os elementos finitos da estrutura ou do meio. Geralmente, os problemas são expressos na forma diferencial (derivadas parciais). Assim a obtenção da forma integral é conseguida através da formulação variacional ou de métodos de resíduos ponderados (BATHE, 1996). A matriz de coeficientes associada com o problema de álgebra linear é uma matriz indefinida (para o piezelétrico), no qual é usado o método de Gauss para obtenção dos deslocamentos mecânicos e elétricos do atuador piezelétrico flextensional.

O projeto é desenvolvido para uma estrutura bidimensional (considerando o 
estado plano de tensões ou deformações), onde o domínio de projeto é discretizado com elementos quadriláteros isoparamétricos de quatro nós, considerando dois graus de liberdade por nó para os elementos não-piezelétricos e três graus de liberdades por nó para os elementos piezelétricos. Nos próximos subitens será feita uma breve abordagem da formulação do elemento isoparamétrico de quatro nós (item 3.4.1) e a matriz de rigidez para o elemento piezelétrico (item 3.4.2), e no último item (item 3.5) a solução do sistema de equações.

\subsubsection{Formulação do elemento isoparamétrico de quatro nós}

Considere a configuração bidimensional do elemento quadrilátero de quatro nós, no sistema de coordenadas locais $(\xi, \eta)$ (Fig. 3.5(a)) para um sistema de coordenadas globais $(x, y)$ (Fig. 3.5(b)).

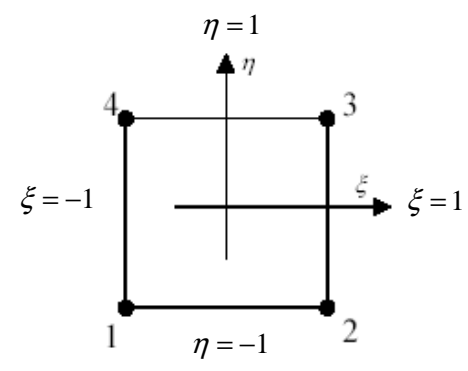

(a)

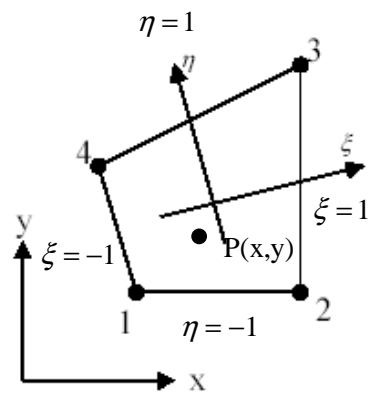

(b)

Figura 3.5- Mapa de coordenadas isoparamétricas. (a) elemento em coordenadas locais, (b) elemento em coordenadas globais.

A formulação de elementos finitos para análise estática e dinâmica de estruturas piezelétricas foi desenvolvida inicialmente por Allik; Hughes (1970). Para análise piezelétrica é necessário formular o elemento com dois graus de liberdade de deslocamento e um grau de liberdade para o potencial elétrico por nó. O 
deslocamento e o potencial podem ser expressos respectivamente para cada elemento como:

$$
\begin{aligned}
& \{\mathbf{u}\}_{e}=\left[\mathbf{N}_{u}\right]^{t}\left\{\mathbf{u}_{e}\right\} \\
& \{\varphi\}_{e}=\left[\mathbf{N}_{\varphi}\right]^{t}\left\{\varphi_{e}\right\}
\end{aligned}
$$

onde:

$$
\begin{aligned}
& \left\{\mathbf{N}_{u}\right\}=\text { função de forma de deslocamento mecânico } \\
& \left\{\mathbf{N}_{\varphi}\right\}=\text { função de forma de deslocamento elétrico } \\
& \left\{\mathbf{u}_{e}\right\}=\text { vetor deslocamento mecânico nodal } \\
& \left\{\varphi_{e}\right\}=\text { vetor potencial elétrico nodal (ou deslocamento elétrico nodal) }
\end{aligned}
$$

No sistema de coordenadas locais, o elemento é quadrado (dimensão $2 \times 2$ ), com o eixo de coordenadas no centro do elemento. Já no sistema de coordenadas globais, o elemento é distorcido da forma retangular. Sendo a função de forma $(\boldsymbol{N})$ função do sistema de coordenadas locais, as coordenadas de qualquer ponto $P$, podem ser expressas em termos das coordenadas $(x, y)$ dos nós, assim:

$$
\left\{\begin{array}{l}
x(\xi, \eta) \\
y(\xi, \eta)
\end{array}\right\}=[\mathbf{N}]\{\mathbf{x}\}_{e}
$$

onde $\{x\}_{e}$ é o vetor de coordenadas nodais do elemento, ou seja: 


$$
\{x\}_{e}=\left\{\begin{array}{llllllll}
x_{1} & y_{1} & x_{2} & y_{2} & x_{3} & y_{3} & x_{4} & y_{4}
\end{array}\right\}
$$

e $[N]$ é a função de forma expressa na forma matricial, como:

$$
[\mathbf{N}]=\left[\begin{array}{cccccccc}
N_{1} & 0 & N_{2} & 0 & N_{3} & 0 & N_{4} & 0 \\
0 & N_{1} & 0 & N_{2} & 0 & N_{3} & 0 & N_{4}
\end{array}\right]
$$

A função de forma são funções de interpolação de Lagrange e são expressas em coordenadas $(\xi, \eta)$ :

$$
\begin{aligned}
& N_{1}(\xi, \eta)=\frac{(1-\xi)(1-\eta)}{4} \\
& N_{2}(\xi, \eta)=\frac{(1+\xi)(1-\eta)}{4} \\
& N_{3}(\xi, \eta)=\frac{(1+\xi)(1+\eta)}{4} \\
& N_{4}(\xi, \eta)=\frac{(1-\xi)(1+\eta)}{4}
\end{aligned}
$$

As coordenadas de um ponto genérico $(x, y)$ do elemento isoparamétrico, mostrado na Fig. 3.5(b), são obtidas em função das coordenadas nodais do elemento, usando os mesmos polinômios bilineares do elemento retangular, Eq. (3.29), dessa forma:

$$
\begin{aligned}
& x=N_{1} x_{1}+N_{2} x_{2}+N_{3} x_{3}+N_{4} x_{4} \\
& y=N_{1} y_{1}+N_{2} y_{2}+N_{3} y_{3}+N_{4} y_{4}
\end{aligned}
$$

A Eq. (3.30), representa as aproximações para as componentes horizontal e vertical, respectivamente, do deslocamento mecânico de cada ponto no domínio do 
elemento. Para o deslocamento elétrico (Eq. (3.31)):

$$
\varphi=N_{1} \varphi_{1}+N_{2} \varphi_{2}+N_{3} \varphi_{3}+N_{4} \varphi_{4}
$$

No item seguinte veremos como determinar a rigidez do elemento.

\subsubsection{Formulação da matriz do elemento}

A expressão geral da matriz do elemento é escrita em termos das coordenadas globais $(x, y)$. Portanto, o diferencial de comprimento $d x$ e $d y$ devem ser expressos em termos do diferencial de coordenadas locais $d \xi$ e $d \eta$. Além do que, a deformação é definida em termos da derivada da função de forma em coordenadas globais, e estas derivadas são os elementos da matriz [B] (Eq. (3.32)) (OSTERGAARD; PAWLAK, 1986) e eles devem ser transformados em derivada na respectiva coordenada local. Portanto, matriz [B] é uma matriz de operadores diferenciais dos polinômios bilineares (Eq. (3.29)).

$$
[\mathbf{B}]=\left[\begin{array}{cccccccccccc}
\frac{\partial N_{1}}{\partial x} & 0 & \frac{\partial N_{2}}{\partial x} & 0 & \frac{\partial N_{3}}{\partial x} & 0 & \frac{\partial N_{4}}{\partial x} & 0 & 0 & 0 & 0 & 0 \\
0 & \frac{\partial N_{1}}{\partial y} & 0 & \frac{\partial N_{2}}{\partial y} & 0 & \frac{\partial N_{3}}{\partial y} & 0 & \frac{\partial N_{4}}{\partial y} & 0 & 0 & 0 & 0 \\
\frac{\partial N_{1}}{\partial y} & \frac{\partial N_{1}}{\partial x} & \frac{\partial N_{2}}{\partial y} & \frac{\partial N_{2}}{\partial x} & \frac{\partial N_{3}}{\partial y} & \frac{\partial N_{3}}{\partial x} & \frac{\partial N_{4}}{\partial y} & \frac{\partial N_{4}}{\partial x} & 0 & 0 & 0 & 0 \\
0 & 0 & 0 & 0 & 0 & 0 & 0 & 0 & \frac{\partial N_{1}}{\partial x} & \frac{\partial N_{2}}{\partial x} & \frac{\partial N_{3}}{\partial x} & \frac{\partial N_{4}}{\partial x} \\
0 & 0 & 0 & 0 & 0 & 0 & 0 & 0 & \frac{\partial N_{1}}{\partial y} & \frac{\partial N_{2}}{\partial y} & \frac{\partial N_{3}}{\partial y} & \frac{\partial N_{4}}{\partial y}
\end{array}\right]
$$

Os diferenciais $(d x, d y)$ são relacionados com os diferenciais $d \xi$ e $d \eta$ por meio da Eq. (3.27). Assim: 


$$
\begin{aligned}
& d x=\frac{\partial x}{\partial \xi} d \xi+\frac{\partial x}{\partial \eta} d \eta \\
& d y=\frac{\partial y}{\partial \xi} d \xi+\frac{\partial y}{\partial \eta} d \eta
\end{aligned}
$$

onde:

$$
\begin{aligned}
& \frac{\partial x}{\partial \xi}=\sum \frac{\partial N_{i}}{\partial \xi} x_{i} ; \quad \frac{\partial y}{\partial \xi}=\sum \frac{\partial N_{i}}{\partial \xi} y_{i} \\
& \frac{\partial x}{\partial \eta}=\sum \frac{\partial N_{i}}{\partial \eta} x_{i} ; \quad \frac{\partial y}{\partial \eta}=\sum \frac{\partial N_{i}}{\partial \eta} y_{i}
\end{aligned}
$$

As derivadas das coordenadas são combinadas na forma matricial como:

$$
[\mathbf{j}]=\left[\begin{array}{ll}
\frac{\partial x}{\partial \xi} & \frac{\partial y}{\partial \xi} \\
\frac{\partial x}{\partial \eta} & \frac{\partial y}{\partial \eta}
\end{array}\right]
$$

onde [j] é a matriz Jacobiana de transformação.

Relacionando as equações (Eqs. (3.33) e (3.35)), os diferenciais de dois sistemas de coordenadas podem ser expressos por:

$$
\left\{\begin{array}{l}
d x \\
d y
\end{array}\right\}=[\mathbf{j}]^{t}\left\{\begin{array}{l}
d \xi \\
d \eta
\end{array}\right\}
$$

De maneira semelhante, as derivadas da função de forma dos nós "i" são relacionadas por: 


$$
\left\{\begin{array}{c}
\frac{\partial \mathbf{N}_{i}}{\partial x} \\
\frac{\partial \mathbf{N}_{i}}{\partial y}
\end{array}\right\}=[\mathbf{j}]^{-1}\left\{\begin{array}{c}
\frac{\partial \mathbf{N}_{i}}{\partial \xi} \\
\frac{\partial \mathbf{N}_{i}}{\partial \eta}
\end{array}\right\}
$$

Assim, podemos escrever a matriz [B] na forma matricial, como:

$$
\begin{aligned}
& \{\mathcal{E}\}_{e}=\left[\mathbf{B}_{u}\right]\left\{\mathbf{u}_{e}\right\} \\
& \{\mathbf{E}\}_{e}=\left[\mathbf{B}_{\varphi}\right]\left\{\varphi_{e}\right\}
\end{aligned}
$$

Substituindo as Eq. (3.38) nas Eqs. constitutivas (3.9), obtemos:

$$
\begin{aligned}
& \{\mathbf{T}\}_{e}=\left[\mathbf{c}^{E}\right]\left[\mathbf{B}_{u}\right]\left\{\mathbf{u}_{e}\right\}+[\mathbf{e}]^{t}\left[\mathbf{B}_{\varphi}\right]\left\{\boldsymbol{\varphi}_{e}\right\} \\
& \{\mathbf{D}\}_{e}=[\mathbf{e}]\left[\mathbf{B}_{u}\right]\left\{\mathbf{u}_{e}\right\}+[\boldsymbol{\varepsilon}]\left[\mathbf{B}_{\varphi}\right]\left\{\varphi_{e}\right\}
\end{aligned}
$$

As equações estáticas de equilíbrio de um material piezelétrico podem ser obtidas do trabalho virtual das forças externas e da variação de energia interna produzidas por deslocamentos virtuais. Assumindo que não são aplicadas forças no corpo ou tração na superfície no elemento, contudo, cargas pontuais nos nós são permitidas, então, os trabalhos virtuais destas cargas pontuais são:

$$
\begin{aligned}
& \int_{V_{e}}\{\delta \mathbf{S}\}_{e}^{t}\{\mathbf{T}\}_{e} d V=\left\{\delta \mathbf{u}_{e}\right\}^{t}\left\{\mathbf{F}_{e}\right\} \\
& -\int_{V_{e}}\{\delta \mathbf{E}\}_{e}^{t}\{\mathbf{D}\}_{e} d V=\left\{\delta \phi_{e}\right\}^{t}\left\{\mathbf{Q}_{e}\right\}
\end{aligned}
$$

As deformações dos deslocamentos virtuais podem ser substituídas por: 


$$
\begin{aligned}
& \{\delta \mathcal{E}\}_{e}^{t}=\left\{\delta \mathbf{u}_{e}\right\}^{t}\left[\mathbf{B}_{u}\right]^{t} \\
& \{\delta \mathbf{E}\}_{e}^{t}=-\left\{\delta \varphi_{e}\right\}^{t}\left[\mathbf{B}_{\varphi}\right]^{t}
\end{aligned}
$$

Substituindo as Eqs (3.39) e (3.41) na Eq. (3.40), podemos escrever:

$$
\begin{aligned}
& \left\{\delta \mathbf{u}_{e}\right\}^{t} \int_{V}\left(\left[\mathbf{B}_{u}\right]^{t}\left[\mathbf{c}^{E}\right]\left[\mathbf{B}_{u}\right]\left\{\mathbf{u}_{e}\right\}+\left[\mathbf{B}_{u}\right]^{t}[\mathbf{e}]^{t}\left[\mathbf{B}_{\varphi}\right]\left\{\varphi_{e}\right\}\right) d V=\left\{\delta \mathbf{u}_{e}\right\}^{t}\left\{\mathbf{F}_{e}\right\} \\
& \left\{\delta \varphi_{e}\right\}^{t} \int_{V}\left(\left[\mathbf{B}_{\varphi}\right]^{t}[\mathbf{e}]\left[\mathbf{B}_{u}\right]\left\{\mathbf{u}_{e}\right\}+\left[\mathbf{B}_{\varphi}\right]^{t}\left[\boldsymbol{\varepsilon}_{D}\right]\left[\mathbf{B}_{\varphi}\right]\left\{\varphi_{e}\right\}\right) d V=\left\{\delta \varphi_{e}\right\}^{t}\left\{\mathbf{Q}_{e}\right\}
\end{aligned}
$$

Integrando no volume do elemento, podemos obter as seguintes matrizes:

$$
\begin{aligned}
& {\left[\mathbf{K}_{u u}\right]_{e}=\int_{V_{e}}\left[\mathbf{B}_{u}\right]^{t}\left[\mathbf{c}^{E}\right]\left[\mathbf{B}_{u}\right] d V} \\
& {\left[\mathbf{K}_{u \varphi}\right]_{e}=\int_{V_{e}}\left[\mathbf{B}_{u}\right]^{t}[\mathbf{e}]^{t}\left[\mathbf{B}_{\varphi}\right] d V} \\
& {\left[\mathbf{K}_{\varphi u}\right]_{e}=\int_{V_{e}}\left[\mathbf{B}_{\varphi}\right]^{t}[\mathbf{e}]\left[\mathbf{B}_{u}\right] d V} \\
& {\left[\mathbf{K}_{\varphi \varphi}\right]_{e}=-\int_{V_{e}}\left[\mathbf{B}_{\varphi}\right]^{t}\left[\boldsymbol{\varepsilon}_{D}\right]\left[\mathbf{B}_{\varphi}\right] d V}
\end{aligned}
$$

Por exemplo, a matriz de rigidez $\left[\mathbf{K}_{\varphi \varphi}\right]_{e}$ refere-se a matriz de rigidez dielétrica do elemento no sistema de equações algébricas lineares (conforme Eq. (3.39)). Usando o Jacobiano (Eq. (3.33)) como um operador para transformar as coordenadas locais $(\xi, \eta)$ em coordenadas globais $(x, y)$, Eqs (3.41 à 3.44) podem ser escritas da seguinte forma: 


$$
[\mathbf{K}]_{e}=h_{e} \int_{-1}^{1} \int_{-1}^{1}[\mathbf{B}]^{t}[\mathbf{C}][\mathbf{B}]|\mathbf{J}| d \eta d \xi
$$

Podemos escrever a equação para o elemento como:

$$
\left[\begin{array} { l l } 
{ [ \mathbf { K } _ { u u } ] } & { [ \mathbf { K } _ { u \varphi } ] } \\
{ [ \mathbf { K } _ { u \varphi } ] } & { [ \mathbf { K } _ { \varphi \varphi } ] ] _ { \mathrm { e } } }
\end{array} \left\{\begin{array}{l}
\mathbf{u} \\
\boldsymbol{\varphi}\}_{\mathrm{e}}
\end{array}=\left\{\begin{array}{l}
\mathbf{F} \\
\mathbf{Q}
\end{array}\right\}_{\mathrm{e}}\right.\right.
$$

A equação acima (Eq. (3.48)) representa a matriz local de um elemento, consistindo num sistema resultante de 12 equações a 12 incógnitas (para o elemento não-piezelétrico, o sistema reduz a 8 equações a 8 incógnitas), onde os coeficientes dependem da integração da Eq. (3.47). A matriz local elasto-piezo-dielétrica mostrada na Eq. (3.48) é inserida numa matriz global [K], através da conectividade de cada elemento, que associa um número a cada elemento e o número de nós a que está conectado. Cada nó por sua vez está representado por uma matriz de coordenadas. Montada a matriz de rigidez global e conhecido o vetor de cargas mecânicas e elétricas aplicada em cada nó, calculamos o vetor de deslocamento nodal a partir da relação de equilíbrio.

$$
\left[\begin{array}{ll}
{\left[\mathbf{K}_{u u}\right]} & {\left[\mathbf{K}_{u \varphi}\right]} \\
{\left[\mathbf{K}_{u \varphi}\right]} & {\left[\mathbf{K}_{\varphi \varphi}\right]}
\end{array}\right]\left\{\begin{array}{l}
\mathbf{u} \\
\boldsymbol{\varphi}
\end{array}\right\}=\left\{\begin{array}{l}
\mathbf{F} \\
\mathbf{Q}
\end{array}\right\}
$$

\subsection{Determinação dos deslocamentos nodais}

Determinada a matriz de rigidez $[\mathbf{K}]$ e sendo conhecido o vetor de forças mecânicas e elétricas ( $\{\mathbf{F}\}$ e $\{\mathbf{Q}\}$ respectivamente) aplicado nos nós, calculamos o 
vetor de deslocamentos nodais $\{\mathbf{u}\}$ e $\{\varphi\}$ a partir da relação de equilíbrio Eq. (3.49). Como a matriz $[\mathbf{K}]$ é uma matriz indefinida (autovalores positivos e negativos), para resolver o sistema de equações lineares, dada pela Eq. (3.49), aplica-se o método da coluna ativa (sub-rotina COLSOL, BATHE, 1996), para se determinar o vetor de deslocamento nodal da estrutura. 


\section{Capítulo 4: Formulação do problema de OT para projetos de atuadores piezelétricos flextensionais}

\subsection{Introdução}

No projeto de atuadores piezelétricos flextensionais, o objetivo é maximizar os deslocamentos gerados (ou forças geradas) em uma região do domínio que deve ser excitado com cargas elétricas (ou potencial elétrico) no atuador. Assim, o atuador deverá combinar uma estrutura flexível para obter uma grande deformação num determinado ponto e com rigidez o suficiente para suportar os trabalhos externos e internos. Então, o problema será formulado em termos de energia e se divide em duas partes: transdução média e a flexibilidade média, estas formulações expressam os deslocamentos elétricos ou mecânicos em qualquer região do meio piezelétrico como uma função dos deslocamentos (obtidos através do MEF) e campo elétrico conhecido, causado por cargas elétricas aplicadas nos eletrodos da piezocerâmica. A transdução média está relacionada com o deslocamento gerado pelo atuador em uma determinada direção e ponto da estrutura, quando a piezocerâmica estiver submetida a um determinado diferencial de potencial (ou cargas elétricas) (item 4.3.1). Já a flexibilidade média está relacionada com a rigidez estrutural do atuador de modo a dar estabilidade estrutural ao atuador (item 4.3.2). No item 4.2 descreveremos o conceito de transdução média para um atuador piezelétrico flextensional. A descrição do problema de otimização para projeto de atuador flextensional piezelétrico será descrita no item 4.4, no qual será abordada a formulação do problema para atender a transdução e a flexibilidade média, através de uma formulação da função multiobjetivo. Por fim, no item 4.5 é apresentada à análise de sensibilidade da função multi-objetivo. 


\subsection{Transdução média.}

Para satisfazer a condição de flexibilidade (máxima deformação) o problema é definido usando-se o conceito de transdução média. Para obtermos a transdução média, aplicaremos o teorema da reciprocidade (ou teorema de Betti) proveniente da teoria da elasticidade (Timoshesko; Goodier, 1980; BARBER, 1992) estendido para o meio piezelétrico. Para ilustrar estes conceitos considere um corpo tridimensional, constituído de material elástico linear, com domínio $\Gamma$ e fixo na região $\Gamma_{u}$, como mostrado na Fig. 4.1. O corpo linear elástico é suportado cinematicamente e sujeito a uma carga $\mathbf{t}_{1}$ na região $\Gamma_{t 1}$ que produz um campo de deslocamentos $\mathbf{u}_{1}$ (Fig. 4.1 caso a), e a carga $\mathbf{t}_{2}$ na região $\Gamma_{t 2}$ que produz um campo de deslocamentos $\mathbf{u}_{2}$ (Fig. 4.1 caso b). Para simplificar a formulação, em ambos os casos, as forças de volume que agem no corpo não são consideradas (embora possam ser consideradas).

Pela teoria da mecânica do meio contínuo, sabe-se que a forma linear da flexibilidade média (ou "mean compliance") pode ser escrito da seguinte forma:

$$
L_{k}\left(\mathbf{u}_{l}\right)=\int_{t_{k}} \mathbf{t}_{k} \cdot \mathbf{u}_{l} d \Gamma \quad \text { para } \mathbf{u}_{l} \in \mathrm{V}
$$

onde $V$ é o espaço linear admissível, tal que $V=\left\{\mathbf{v}=v_{i} \mathbf{e}_{i}: v_{i} \in H_{1}(\Omega)\right.$ com $\mathbf{v}=0$ em $\left.\Gamma_{u}, i=1,2,3\right\} ; \mathbf{t}=\mathbf{T}$.n, sendo $\boldsymbol{T}$ o tensor tensão (definição do estado de tensão num dado ponto) e $\boldsymbol{n}$ um vetor normal à superfície $\Gamma_{t^{k}}$; os índices assumem o valor 1 para o primeiro caso de carga ou 2 para o segundo. 


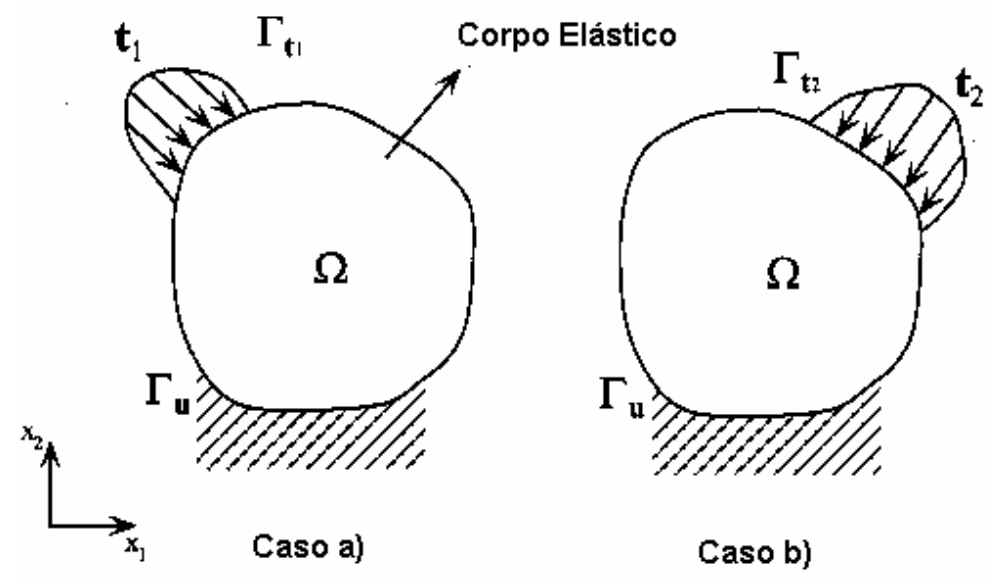

Figura 4.1- Teorema da reciprocidade na elasticidade.

Para atender aos requisitos cinemáticos do atuador flextensional piezelétrico precisamos conhecer também a transdução média. Então, primeiramente vamos definir o conceito de energia mútua e estendê-la para transdução média usando o teorema da reciprocidade, bem conhecida da teoria elástica. Como ilustrado na Fig. 4.1, podemos obter:

$$
\int_{\Gamma_{t 1}} \mathbf{t}_{1} \cdot \mathbf{u}_{2} d \Gamma=\int_{\Gamma_{t 2}} \mathbf{t}_{2} \cdot \mathbf{u}_{1} d \Gamma
$$

onde $\int_{\Gamma_{t}} \mathbf{t} \cdot \mathbf{u} d \Gamma=\int_{\Gamma_{t}} t_{i} u_{i} d \Gamma, \mathbf{t}=\mathbf{T} \cdot \mathbf{n}, \boldsymbol{T}$ é o tensor das tensões, e $\boldsymbol{n}$ é o vetor normal a superfície.

A relação anterior pode ser provada considerando duas situações:

- a carga $\mathbf{t}_{1}$ é aplicado à superfície $\Gamma_{t 1}$, e permanece constante enquanto a carga $\mathbf{t}_{2}$ é aplicada gradualmente na superfície $\Gamma_{t 2}$. Neste caso, o trabalho total realizado é dado por: 


$$
\Pi_{1}=\frac{1}{2} \int_{\Gamma_{t 1}} \mathbf{t}_{1} \cdot \mathbf{u}_{1} d \Gamma+\frac{1}{2} \int_{\Gamma_{t 2}} \mathbf{t}_{2} \cdot \mathbf{u}_{2} d \Gamma+\int_{\Gamma_{t 1}} \mathbf{t}_{1} \cdot \mathbf{u}_{2} d \Gamma
$$

- nesta situação, os carregamentos são aplicados na ordem reversa em relação a situação anterior, ou seja, a carga $\mathbf{t}_{2}$ é aplicada na superfície $\Gamma_{t 2}$, e permanece constante enquanto a carga $\mathbf{t}_{1}$ é aplicado gradualmente na superfície $\Gamma_{t 1}$. Neste caso, o trabalho total realizado é dado por:

$$
\Pi_{2}=\frac{1}{2} \int_{\Gamma_{t 2}} \mathbf{t}_{2} \cdot \mathbf{u}_{2} d \Gamma+\frac{1}{2} \int_{\Gamma_{t 1}} \mathbf{t}_{1} \cdot \mathbf{u}_{1} d \Gamma+\int_{\Gamma_{t 2}} \mathbf{t}_{2} \cdot \mathbf{u}_{1} d \Gamma
$$

O resultado final em cada caso são iguais, $\Pi_{1}=\Pi_{2}$, e portanto, comparando as duas expressões, obtemos o mesmo resultado da Eq. (4.2). Este teorema é uma generalização do Teorema de Maxwell, o qual é específico para o caso de aplicação de forças pontuais, ao invés de um sistema de forças superficiais. Este teorema é muito útil quando queremos determinar o campo de deslocamentos provocado pela força aplicada num dado ponto, conhecendo-se a força e o campo de deslocamentos que atuam em outro ponto de uma estrutura.

Agora, considerando a equação do equilíbrio para o meio elástico:

$$
\int_{\Omega} \varepsilon(\mathbf{u})^{t} \mathbf{c} \varepsilon(\mathbf{v}) d \Omega=\int_{\Gamma_{t}} \mathbf{t} \cdot \mathbf{v} d \Omega \quad \text { para } \mathbf{u} \in \hat{\mathrm{V}} \text { e } \forall \mathbf{v} \in \hat{\mathrm{V}}
$$

onde $\hat{V}=\left\{\mathbf{v}=v_{i} \overline{\mathbf{e}}_{i}: v_{i} \in H^{1}(\Omega)\right.$ com $\mathbf{v}=0$ em $\left.\Gamma_{\mathbf{u}}\right\}, \Omega$ é o domínio do meio elástico, c é a matriz de propriedades elásticas, "t" é a notação de matriz transposta, e 


$$
\varepsilon_{i j}(\mathbf{u})=\frac{1}{2}\left(\frac{\partial u_{i}}{\partial x_{j}}+\frac{\partial u_{j}}{\partial x_{i}}\right)
$$

e $\varepsilon_{i j}$ é a matriz de deformações infinitesimais, então temos:

$$
\begin{aligned}
& \int_{\Omega} \varepsilon\left(\mathbf{u}_{1}\right)^{t} \mathbf{c} \varepsilon\left(\mathbf{u}_{2}\right) d \Omega=\int_{\Gamma_{t 1}} \mathbf{t}_{1} \cdot \mathbf{u}_{2} d \Omega \\
& \int_{\Omega} \varepsilon\left(\mathbf{u}_{2}\right)^{t} \mathbf{c} \varepsilon\left(\mathbf{u}_{1}\right) d \Omega=\int_{\Gamma_{t 1}} \mathbf{t}_{2} \cdot \mathbf{u}_{1} d \Omega
\end{aligned}
$$

Portanto, podemos escrever a equação (4.2) na forma:

$$
\begin{aligned}
& L_{1}\left(\mathbf{u}_{2}\right)=\int_{\Gamma_{t 1}} \mathbf{t}_{1} \mathbf{u}_{2} d \Gamma=\int_{\Omega} \varepsilon\left(\mathbf{u}_{1}\right)^{t} \mathbf{c} \varepsilon\left(\mathbf{u}_{2}\right) d \Omega= \\
& \int_{\Omega} \varepsilon\left(\mathbf{u}_{2}\right)^{t} \mathbf{c} \varepsilon\left(\mathbf{u}_{1}\right) d \Omega=\int_{\Gamma_{t 2}} \mathbf{t}_{2} \cdot \mathbf{u}_{1} d \Gamma=L_{2}\left(\mathbf{u}_{1}\right)
\end{aligned}
$$

onde $L_{1}$ (ou $L_{2}$ ) é definido como a energia mútua do sistema, entre $\Gamma_{t 1} e \Gamma_{\mathrm{t} 2}$. Se assumirmos por exemplo um valor unitário para o carregamento $\mathbf{t}_{2}$, a energia mútua representa o quanto se desloca a região $\Gamma_{t 2}$ devido à ação do carregamento $\mathbf{t}_{1}$, aplicado na região $\Gamma_{t 1}$. Ou seja, a energia mútua está relacionada com o deslocamento do corpo em um ponto e direção de interesse, quando submetido a um carregamento em outro ponto distinto. Portanto, se quisermos a máxima deflexão em qualquer ponto do corpo, por exemplo, devemos maximizar a energia mútua correspondente a esses pontos.

Estendendo o teorema da reciprocidade para o meio piezelétrico, seguem-se os mesmos passos. Considere um corpo piezelétrico mostrado na Fig. 4.2, sujeito a uma carga elétrica $\mathbf{d}_{1}$ e $\mathbf{d}_{2}$ aplicados no contorno de $\Gamma_{d 1}$ e $\Gamma_{\mathrm{d} 2}$, respectivamente e trações $\mathbf{t}_{1}$ e $\mathbf{t}_{2}$ aplicados nos contornos de $\Gamma_{d 1}$ e $\Gamma_{\mathrm{d} 2}$, respectivamente. 
Primeiramente, aplicamos a carga $\mathbf{t}_{1}$ e a carga elétrica $\mathbf{d}_{1}$ na superfície, simultaneamente e mantendo-os constantes enquanto a carga $\mathbf{t}_{2}$ e a carga elétrica $\mathbf{d}_{2}$ na superfície são aplicados simultaneamente no meio, então, o trabalho total (mecânico e elétrico) feito pelas cargas na superfície é dado pela expressão:

$$
\begin{aligned}
\Pi_{1}= & \frac{1}{2} \int_{\Gamma_{t 1}} \mathbf{t}_{1} \cdot \mathbf{u}_{1} d \Gamma+\frac{1}{2} \int_{\Gamma_{d 1}} \mathbf{d}_{1} \phi_{1} d \Gamma+\frac{1}{2} \int_{\Gamma_{t 1}} \mathbf{t}_{1} \cdot \mathbf{u}_{2} d \Gamma+ \\
& +\int_{\Gamma_{d 1}} \mathbf{d}_{1} \phi_{2} d \Gamma+\frac{1}{2} \int_{\Gamma_{t 2}} \mathbf{t}_{2} \cdot \mathbf{u}_{2} d \Gamma+\frac{1}{2} \int_{\Gamma_{d 2}} \mathbf{d}_{2} \phi_{2} d \Gamma
\end{aligned}
$$

onde $\mathbf{d}=\mathbf{D} \cdot \mathbf{n}, \boldsymbol{D}$ é vetor cargas elétricas, e $\boldsymbol{n}$ é o vetor normal à superfície.
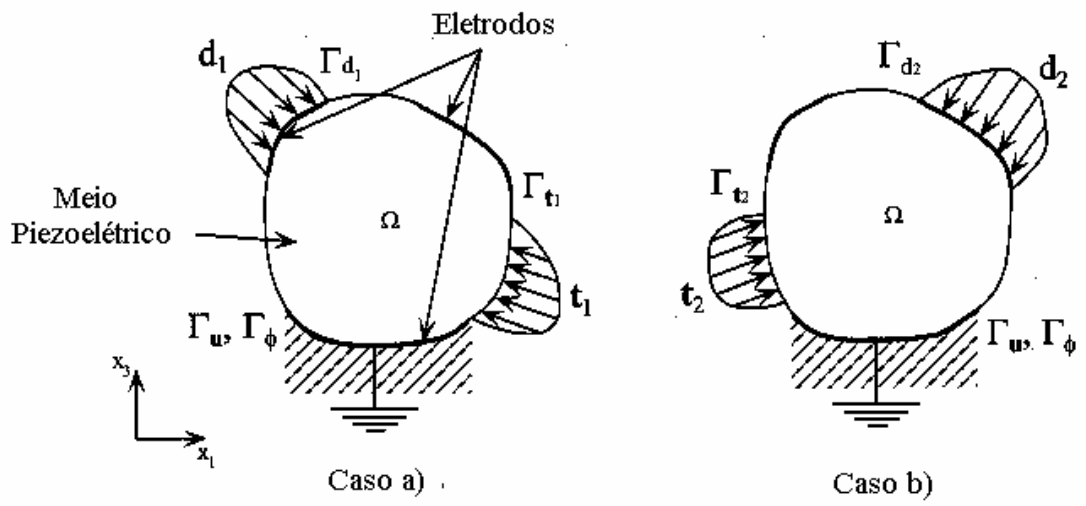

Figura 4.2- Uma extensão do teorema da reciprocidade para a piezeletricidade.

Agora, se aplicarmos a carga mecânica e a carga elétrica na superfície ao contrário do caso anterior, ou seja, primeiro aplicamos a carga $\mathbf{t}_{2}$ e a carga elétrica $\mathbf{d}_{2}$ na superfície, simultaneamente e mantendo-os constantes enquanto a carga $\mathbf{t}_{1}$ e a carga elétrica $\mathbf{d}_{1}$ na superfície são aplicados simultaneamente no meio, então, o trabalho total (mecânico e elétrico) feito pelas cargas na superfície é dado pela expressão: 


$$
\begin{aligned}
\Pi_{2}= & \frac{1}{2} \int_{\Gamma 2} \mathbf{t}_{2} \cdot \mathbf{u}_{2} d \Gamma+\frac{1}{2} \int_{\Gamma_{d 2}} \mathbf{d}_{2} \phi_{2} d \Gamma+\frac{1}{2} \int_{\Gamma_{t 2}} \mathbf{t}_{2} \cdot \mathbf{u}_{1} d \Gamma+ \\
& +\int_{\Gamma_{d 2}} \mathbf{d}_{2} \phi_{1} d \Gamma+\frac{1}{2} \int_{\Gamma_{t 1}} \mathbf{t}_{1} \cdot \mathbf{u}_{1} d \Gamma+\frac{1}{2} \int_{\Gamma_{d 1}} \mathbf{d}_{1} \phi_{1} d \Gamma
\end{aligned}
$$

Em cada caso, desde que o carregamento e a carga elétrica na superfície sejam aplicados gradativamente, o trabalho feito será armazenado como energia de deformação e elétrica no corpo. Como o estado final para cada caso é idêntico, concluí-se que $\Pi_{1}=\Pi_{2}$, e portanto, obtemos:

$$
\begin{aligned}
L_{1}\left(\mathbf{u}_{2}, \phi_{2}\right) & =\int_{\Gamma_{\Gamma_{11}}} \mathbf{t}_{1} \cdot \mathbf{u}_{2} d \Gamma+\int_{\Gamma_{d 1}} \mathbf{d}_{1} \phi_{2} d \Gamma+ \\
& =\int_{\Gamma_{t 2}} \mathbf{t}_{2} \cdot \mathbf{u}_{1} d \Gamma+\int_{\Gamma_{d 2}} \mathbf{d}_{2} \phi_{1} d \Gamma=L_{2}\left(\mathbf{u}_{1}, \phi_{1}\right)
\end{aligned}
$$

que é o teorema da reciprocidade estendido para o meio piezelétrico. Analogamente à energia mútua $L_{1}\left(\mathbf{u}_{2}, \phi_{2}\right)$ (ou $\left.L_{2}\left(\mathbf{u}_{1}, \phi_{1}\right)\right)$ será denominada transdução média visto que a quantidade mecânica e elétrica estão envolvidas (Fig. 4.2).

Agora considerando a formulação anterior da equação de equilíbrio do meio piezelétrico, temos:

$$
\begin{aligned}
& \int_{\Omega} \varepsilon(\mathbf{u})^{t} \mathbf{c}^{E}(\mathbf{v}) d \Omega+\int_{\Omega}(\nabla \phi)^{t} \mathbf{e}^{t} \mathcal{E}(\mathbf{v}) d \Omega=\int_{\Gamma_{t}} \mathbf{t} \cdot \mathbf{v} d \Gamma \\
& \int_{\Omega} \varepsilon(\mathbf{u})^{t} \mathbf{e} \nabla \phi d \Omega-\int_{\Omega}(\nabla \phi)^{t} \mathcal{E}_{D}^{S} \nabla \phi d \Omega=\int_{\Gamma_{d}} d \phi d \Gamma \\
& \text { para } \mathbf{u}, \phi \in V e \forall \mathbf{v}, \forall \phi \in V
\end{aligned}
$$

onde $\quad \mathrm{V}=\left\{\mathbf{v}=v_{\mathrm{i}} \overline{\mathbf{e}}_{i}, \varphi: v_{\mathrm{i}}, \varphi \in \mathrm{H}^{1}(\Omega) \operatorname{com} \mathbf{v}=0\right.$ em $\Gamma_{\text {u }}$ e $\varphi=0$ em $\Gamma_{\phi}, i=1$ ou 3$\}, \Omega$ agora é o domínio do meio piezelétrico (também pode conter um material não piezelétrico), $\nabla$ é o operador gradiente, e $\mathbf{c}^{E}$, e e $\boldsymbol{\varepsilon}_{D}^{S}$ são as propriedades, elásticas, piezelétricas e dielétricas, respectivamente do meio (descritas no capítulo 3). O 
índice $i$ assume o valor 1 ou 3, porque o problema está considerando o plano 1-3. A piezocerâmica está polarizada na direção \#3.

Escrever a Eq. (4.12) na forma mais compacta definimos os seguintes operadores:

$$
\begin{array}{ll}
A(\mathbf{u}, \mathbf{v})=\int_{\Omega} \varepsilon(\mathbf{u})^{t} \mathbf{c}^{E}(\mathbf{v}) d \Omega & B(\phi, \mathbf{v})=\int_{\Omega}(\nabla \phi)^{t} \mathbf{e}^{t} \varepsilon(\mathbf{v}) d \Omega \\
C(\phi, \varphi)=\int_{\Omega}(\nabla \phi)^{t} \mathbf{\varepsilon}_{D}^{S} \nabla \varphi d \Omega & L_{t}(\mathbf{t}, \mathbf{v})=\int_{\Gamma_{t}} \mathbf{t} \cdot \mathbf{v} d \Gamma \\
L_{d}(\mathbf{d}, \varphi)=\int_{\Gamma_{d}} d \varphi d \Gamma &
\end{array}
$$

Portanto, Eq. (4.12) torna-se:

$$
\begin{aligned}
& A(\mathbf{u}, \mathbf{v})+B(\phi, \mathbf{v})=L_{t}(\mathbf{t}, \mathbf{v}) \\
& B(\varphi, \mathbf{u})-C(\phi, \varphi)=L_{d}(\mathbf{d}, \varphi)
\end{aligned}
$$

Fazendo $\mathbf{u}, \mathbf{v}$ igual a $\mathbf{u}_{1}$ e $\mathbf{u}_{2}, \mathbf{t}$ igual a $\mathbf{t}_{1}$ e $\mathbf{t}_{2}, \mathbf{d}$ igual a $\mathbf{d}_{1}$ e $\mathbf{d}_{2}$, e $\phi, \varphi$ igual a $\phi_{1}$ e $\phi_{2}$, nos temos:

$$
\begin{aligned}
& A\left(\mathbf{u}_{1}, \mathbf{u}_{2}\right)+B\left(\phi_{1}, \mathbf{u}_{2}\right)=L_{t}\left(\mathbf{t}_{1}, \mathbf{u}_{2}\right) \\
& B\left(\phi_{2}, \mathbf{u}_{1}\right)-C\left(\phi_{1}, \phi_{2}\right)=L_{d}\left(\mathbf{d}_{1}, \phi_{2}\right)
\end{aligned}
$$

e:

$$
\begin{aligned}
& A\left(\mathbf{u}_{2}, \mathbf{u}_{1}\right)+B\left(\phi_{2}, \mathbf{u}_{1}\right)=L_{t}\left(\mathbf{t}_{2}, \mathbf{u}_{1}\right) \\
& B\left(\phi_{1}, \mathbf{u}_{2}\right)-C\left(\phi_{2}, \phi_{1}\right)=L_{d}\left(\mathbf{d}_{2}, \phi_{1}\right)
\end{aligned}
$$

Portanto, substituindo estas equações na Eq. (4.11), podemos escrevê-las na forma: 


$$
\begin{aligned}
L_{1}\left(\mathbf{u}_{2}, \phi_{2}\right) & =A\left(\mathbf{u}_{1}, \mathbf{u}_{2}\right)+B\left(\phi_{1}, \mathbf{u}_{2}\right)+B\left(\phi_{2}, \mathbf{u}_{1}\right)-C\left(\phi_{1}, \phi_{2}\right)= \\
& =A\left(\mathbf{u}_{2}, \mathbf{u}_{1}\right)+B\left(\phi_{2}, \mathbf{u}_{1}\right)+B\left(\phi_{1}, \mathbf{u}_{2}\right)-C\left(\phi_{2}, \phi_{1}\right)= \\
& =L_{2}\left(\mathbf{u}_{1}, \phi_{1}\right)
\end{aligned}
$$

Os operadores definidos na Eq. (4.13), bem como a transdução média (Eq. (4.17)) são operadores auto-adjuntos, ou seja, possuem as propriedades definidas pela seguinte expressão:

$$
(u, \mathbf{L} v)=(v, \mathbf{L} u)
$$

onde:

$$
(u, \mathbf{L} v)=\int_{0}^{L} u \mathbf{L} v d x
$$

Agora, aplicando a formulação MEF para um domínio discretizado $\Omega$ (deslocamento e o potencial elétrico são aproximados usando função de forma), a transdução média para o meio piezelétrico pode ser escrita na seguinte forma matricial:

$$
\begin{aligned}
L_{1}\left(\mathbf{U}_{2}, \phi_{2}\right) & =\left\{\mathbf{U}_{2}\right\}^{t}\left\{\mathbf{F}_{1}\right\}+\left\{\phi_{2}\right\}^{t}\left\{\mathbf{Q}_{1}\right\}= \\
& =\left\{\begin{array}{c}
\mathbf{U}_{2}^{t} \\
\boldsymbol{\phi}_{2}^{t}
\end{array}\right\}^{t}\left[\begin{array}{cc}
\mathbf{K}_{\mathbf{u u}} & \mathbf{K}_{\mathbf{u \varphi}} \\
\mathbf{K}_{\mathbf{u \varphi}} & -\mathbf{K}_{\mathbf{\varphi \varphi \varphi}}
\end{array}\right]\left\{\begin{array}{l}
\mathbf{U}_{1} \\
\phi_{1}
\end{array}\right\}=\left\{\mathbf{W}_{2}\right\}^{t}[\overline{\mathbf{K}}]\left\{\mathbf{W}_{1}\right\}= \\
& =\left\{\mathbf{W}_{1}\right\}^{t}[\overline{\mathbf{K}}]\left\{\mathbf{W}_{2}\right\}=\left\{\begin{array}{l}
\mathbf{U}_{1}^{t} \\
\phi_{1}^{t}
\end{array}\right\}^{t}\left[\begin{array}{cc}
\mathbf{K}_{\mathbf{u u}} & \mathbf{K}_{\mathbf{u \varphi}} \\
\mathbf{K}_{\mathbf{u p}} & -\mathbf{K}_{\mathbf{\varphi \varphi \varphi}}
\end{array}\right]\left\{\begin{array}{l}
\mathbf{U}_{2} \\
\phi_{2}
\end{array}\right\}= \\
& =\left\{\mathbf{U}_{1}\right\}^{t}\left\{\mathbf{F}_{2}\right\}+\left\{\phi_{1}\right\}^{t}\left\{\mathbf{Q}_{2}\right\}=L_{2}\left(\mathbf{U}_{1}, \phi_{1}\right)
\end{aligned}
$$

onde $\mathbf{U}_{i}, \phi_{i}, \mathbf{F}_{i}$, e $\mathbf{Q}_{i},(i=1,2)$ são o deslocamento mecânico nodal, potencial elétrico 
nodal, forças mecânicas nodais e cargas elétricas nodais, respectivamente. A matriz $\mathbf{K}_{\mathbf{u u}}, \mathbf{K}_{\mathbf{u} \phi}$ e $\mathbf{K}_{\phi \phi}$ foram definidos no capítulo 3 (item 3.5).

A Eq. (4.20) possibilita a implementar o conceito de transdução média para o meio piezelétrico usando a formulação do MEF em um domínio discretizado. Assim, podemos expressar o deslocamento ou potencial elétrico em algumas regiões do meio piezelétrico como função do deslocamento e campo elétrico, ambos conhecidos, causado por cargas elétricas aplicadas na superfície.

\subsection{Descrição do problema de otimização para projeto de atuador piezelétrico flextensional.}

O projeto de atuadores piezelétricos flextensionais usando a otimização topológica, deve atender à função eletromecânica e à função estrutural. A função eletromecânica está relacionada com o deslocamento gerado em uma determinada direção e região da estrutura flexível devido à carga elétrica aplicada numa determinada região da piezocerâmica. À função estrutural está relacionada com a rigidez estrutural, para garantir a forma do atuador quando sujeito ao trabalho das forças externas. Então, o problema de otimização para o projeto de atuadores piezelétricos flextensionais requer duas diferentes funções objetivo. Uma é relacionado à função eletromecânica (transdução média) e a outra à função estrutural (flexibilidade média), como descritos a seguir.

\subsubsection{Formulação do problema para atender à função eletromecânica}

A função eletromecânica provém da conversão eletromecânica entre duas regiões (ver Fig. 4.3), $\Gamma_{d_{1}}$ (carga elétrica) e $\Gamma_{t_{2}}$ (tração mecânica), do domínio de projeto, ou seja, quanto maior esta função, maior é o deslocamento gerado em 
determinada direção da região $\Gamma_{t_{2}}$ devido carga elétrica aplicada na região $\Gamma_{d_{1}}$. A maximização desta função é obtida pela maximização da transdução média definida na Eq. (4.11), considerando que o caso de carga para a transdução média (caso (a) da Fig. 4.3) é subdividido em dois casos de carga, que são: o primeiro está relacionado com resposta do atuador devido a aplicação da carga de superfície na superfície $\Gamma_{d_{1}}$ do transdutor (caso (a1), ver Fig. 4.3); e o segundo está relacionado com uma simulação de carga aplicada $\mathbf{t}_{2}$ na região $\Gamma_{t_{2}}$ (caso (a2), ver Fig. 4.3), e alguns deslocamentos gerados em direções desejadas.

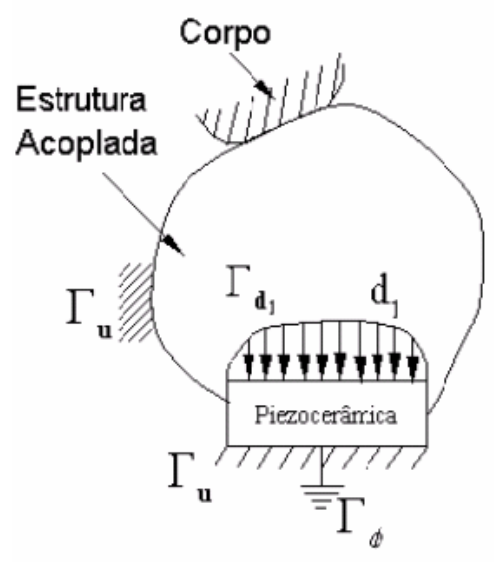

(a1)

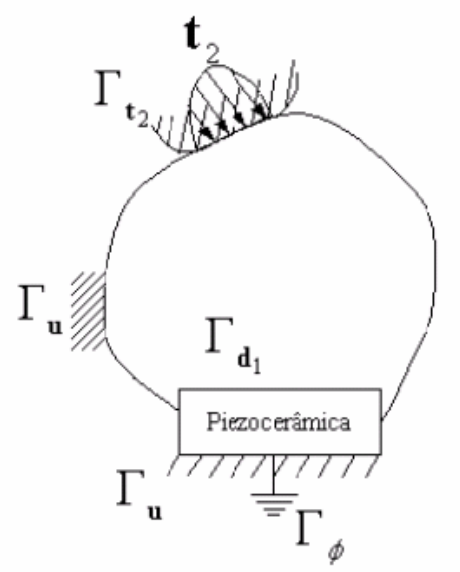

(a2)

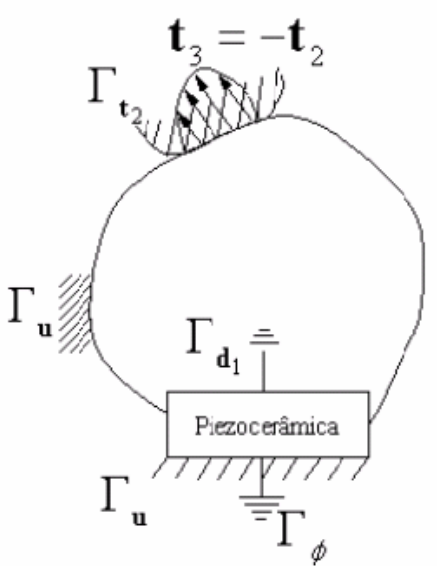

caso (b)

Figura 4.3- Casos de carga para cálculo da transdução média (caso a) e flexibilidade média (caso b).

Considerando Eq. (4.18), como a tração mecânica é nula no caso (a1) $\left(t_{1}=0\right.$, ver Fig. 4.3) e a carga de superfície são nulas no caso (a2) $\left(d_{2}=0\right.$, ver Fig. 4.3), a transdução média entre regiões $\Gamma_{d_{1}}$ e $\Gamma_{t_{2}}$ são dadas por (SILVA; NISHIWAKI; KIKUCHI, 1999): 


$$
\begin{aligned}
L_{2}\left(\mathbf{u}_{1}, \phi_{1}\right) & =L_{t}\left(\mathbf{t}_{2}, \mathbf{u}_{1}\right)=L_{d}\left(d_{1}, \phi_{2}\right)=A\left(\mathbf{u}_{1}, \mathbf{u}_{2}\right)+ \\
& +B\left(\phi_{1}, \mathbf{u}_{2}\right)+B\left(\phi_{2}, \mathbf{u}_{1}\right)-C\left(\phi_{1}, \phi_{2}\right)= \\
& =B\left(\phi_{2}, \mathbf{u}_{1}\right)-C\left(\phi_{1}, \phi_{2}\right)
\end{aligned}
$$

Usando a formulação matricial MEF definido no domínio discretizado:

$$
\begin{aligned}
& L_{2}\left(\mathbf{U}_{1}, \phi_{1}\right)=\left\{\mathbf{U}_{1}\right\}^{t}\left\{\mathbf{F}_{2}\right\}=\left\{\phi_{2}\right\}^{t}\left\{\mathbf{Q}_{1}\right\}=\left\{\begin{array}{c}
\mathbf{U}_{2}^{t} \\
\phi_{2}^{t}
\end{array}\right\}^{t}\left[\begin{array}{ll}
\mathbf{K}_{\mathbf{u u}} & \mathbf{K}_{\mathbf{u} \phi} \\
\mathbf{K}_{\mathbf{u} \phi} & \mathbf{K}_{\phi \phi}
\end{array}\right]\left\{\begin{array}{c}
\mathbf{U}_{1} \\
\phi_{1}
\end{array}\right\} \\
& =\left\{\mathbf{U}_{1}\right\}^{t}\left[\mathbf{K}_{\mathbf{u} \phi}\right]\left\{\phi_{2}\right\}-\left\{\phi_{1}\right\}^{t}\left[\mathbf{K}_{\phi \phi}\right]\left\{\phi_{1}\right\}
\end{aligned}
$$

uma vez que $\left\{\phi_{1}\right\}^{t}\left\{\mathbf{Q}_{2}\right\}=0$.

Portanto, a maximização da transdução média é obtido pela solução do problema de otimização (SILVA; NISHIWAKI; KIKUCHI, 1999):

$$
\begin{array}{ll}
\text { Maximizar: } & \mathrm{L}_{2}\left(\mathbf{u}_{1}, \phi_{1}\right)=\mathrm{L}_{\mathrm{t}}\left(\mathbf{t}_{2}, \mathbf{u}_{1}\right)=\int_{\Gamma_{\mathrm{t}_{2}}} \mathbf{t}_{2} \cdot \mathbf{u}_{1} d \Gamma \\
\text { tal que }: & \mathrm{A}\left(\mathrm{u}_{1}, \mathbf{v}_{1}\right)+\mathrm{B}\left(\phi_{1}, \mathbf{v}_{1}\right)=0 \\
& \mathrm{~B}\left(\varphi_{1}, \mathbf{u}_{1}\right)-\mathrm{C}\left(\phi_{1}, \varphi_{1}\right)=\mathrm{L}_{\mathrm{d}}\left(\mathrm{d}_{1}, \varphi_{1}\right) \\
& \operatorname{para} \mathbf{u}_{1}, \phi_{1} \in \mathrm{V}_{\mathrm{a}} \mathrm{e} \forall \mathbf{v}_{1}, \forall \varphi_{1} \in \mathrm{V}_{\mathrm{a}} \\
& \mathrm{A}\left(\mathbf{u}_{2}, \mathbf{v}_{2}\right)+\mathrm{B}\left(\phi_{2}, \mathbf{v}_{2}\right)=\mathrm{L}_{\mathrm{d}}\left(\mathrm{d}_{1}, \varphi_{1}\right) \\
& \mathrm{B}\left(\varphi_{2}, \mathbf{u}_{2}\right)-\mathrm{C}\left(\phi_{2}, \varphi_{2}\right)=0 \\
& \operatorname{para} \mathbf{u}_{2}, \phi_{2} \in \mathrm{V}_{\mathrm{a}} \mathrm{e} \forall \mathbf{v}_{2}, \forall \varphi_{2} \in \mathrm{V}_{\mathrm{a}} \\
& \int_{\Omega} \rho d \Omega \leq \Omega_{s} \\
0 \leq \rho \leq 1
\end{array}
$$

onde $\mathrm{V}_{\mathrm{a}}=\left\{\mathbf{v}=v_{i} \overline{\mathbf{e}}_{i}, \varphi: v_{i}, \varphi \in \mathrm{H}^{1}(\Omega) \operatorname{com} \mathbf{v}=0\right.$ em $\Gamma_{\mathbf{u}}$ e $\varphi=0$ em $\Gamma_{\phi}, i=1$ ou 3$\}$. $S$ é o domínio de projeto $\Omega$. As outras constantes são condições de equilíbrio e 
restrições de simetria do modelo. A constante relacionada à condição de simetria pode ser destinada a reduzir o custo computacional (que permite aumentar a

discretização). A $\int_{\Omega} \rho d \Omega \leq \Omega_{s}$ restringe a quantidade de material do domínio inicial de projeto, sendo $\Omega_{s}$ o volume final desejado para o domínio e $\rho$ é a função pseudo-densidade do material utilizado no projeto. O problema de otimização acima mencionado é definido na forma contínua; contudo, uma vez que o domínio é discretizado em elementos finitos, a definição mencionada deve ser substituída por uma discretização equivalente (incluindo as equações de equilíbrio).

\subsubsection{Formulação do problema para atender à função estrutural}

Contudo, se considerarmos somente a maximização da transdução média, a solução ótima obtida para esta função poder ser uma estrutura "vazia", isto é, uma estrutura com nenhuma rigidez. Portanto, a função estrutural deve ser definida para fornecer suficiente rigidez entre $\Gamma_{t_{2}}$ e $\Gamma_{d_{1}}$, que assegura a existência de estrutura entre as regiões descritas. Além do que, o atuador precisa gerar força e resistir à reação desta força gerada por qualquer parte da superfície enquanto o atuador estiver atuando ou movendo-se, isto é, o atuador deverá manter a deflexão na região $\Gamma_{t_{2}}$ enquanto ele está sujeito a uma carga elétrica superficial $d_{1}$ na região $\Gamma_{d_{1}}$ (ver Fig. 4.3 caso (b)). Estes objetivos são alcançados resolvendo o problema de otimização relacionado com a minimização da flexibilidade média na região onde há o contato entre o atuador e o corpo, ou seja, a estrutura flexível deve prover rigidez o suficiente para suportar o esforço de reação ao segurar ou mover o corpo, considerando também a superfície da piezocerâmica onde os eletrodos estão aterrados. A flexibilidade média (ou trabalho das forças externas) para o meio piezelétrico é expressa como: 


$$
\begin{aligned}
& L_{3}\left(\mathbf{u}_{3}, \phi_{3}\right)=\int_{\Gamma_{t_{2}}} \mathbf{t}_{3} \cdot \mathbf{u}_{3} d \Gamma=A\left(\mathbf{u}_{3}, \mathbf{u}_{3}\right)+B\left(\phi_{3}, \mathbf{u}_{3}\right)+B\left(\phi_{3}, \mathbf{u}_{3}\right)+ \\
& -C\left(\phi_{3}, \phi_{3}\right)=A\left(\mathbf{u}_{3}, \mathbf{u}_{3}\right)+B\left(\phi_{3}, \mathbf{u}_{3}\right)
\end{aligned}
$$

uma vez que $\mathrm{d}_{3}=0$ (Fig. 4.3 caso (b)). Considerando a formulação do MEF na discretização do domínio, temos:

$$
\begin{aligned}
& L_{3}\left(\mathbf{u}_{3}, \phi_{3}\right)=\left\{\mathbf{U}_{3}\right\}^{t}\left\{\mathbf{F}_{3}\right\}=\left\{\mathbf{U}_{3}\right\}^{t}\left[\mathbf{K}_{\mathbf{u u}}\right]\left\{\mathbf{U}_{3}\right\}+ \\
& +\left\{\mathbf{U}_{3}\right\}^{t}\left[\mathbf{K}_{\mathbf{u} \phi}^{t}\right]\left\{\phi_{3}\right\}=\left\{\begin{array}{c}
\mathbf{U}_{3}^{t} \\
\phi_{3}^{t}
\end{array}\right\}\left[\begin{array}{ll}
\mathbf{K}_{\mathbf{u u}} & \mathbf{K}_{\mathbf{u} \phi} \\
\mathbf{K}_{\mathbf{u} \phi} & \mathbf{K}_{\phi \phi}
\end{array}\right]\left\{\begin{array}{c}
\mathbf{U}_{3} \\
\phi_{3}
\end{array}\right\}
\end{aligned}
$$

uma vez que $\left\{\phi_{3}\right\}^{\mathrm{t}}\left\{\mathbf{Q}_{3}\right\}=\mathbf{0}$. Portanto a minimização da flexibilidade média necessita resolver o problema de otimização (definido na forma contínua):

$$
\begin{array}{ll}
\text { Minimizar: } & \mathrm{L}_{3}\left(\mathbf{u}_{3}, \phi_{3}\right)=\int_{\Gamma_{\mathrm{t}_{3}}} \mathbf{t}_{3} \cdot \mathbf{u}_{3} d \Gamma \\
\text { tal que }: & \mathbf{t}_{3}=-\mathbf{t}_{2} \quad\left(\Gamma_{\mathrm{t}_{3}}=\Gamma_{\mathrm{t}_{2}}\right) \\
& \mathrm{A}\left(\mathbf{u}_{3}, \mathbf{v}_{3}\right)+\mathrm{B}\left(\phi_{3}, \mathbf{v}_{3}\right)=\mathrm{L}_{\mathrm{t}}\left(\mathbf{t}_{3}, \mathbf{v}_{3}\right) \\
& \mathrm{B}\left(\varphi_{3}, \mathbf{u}_{3}\right)-\mathrm{C}\left(\phi_{3}, \varphi_{3}\right)=0 \\
& \operatorname{para} \mathbf{u}_{3}, \phi_{3} \in \mathrm{V}_{\mathrm{b}} \mathrm{e} \forall \mathbf{v}_{3}, \forall \varphi_{3} \in \mathrm{V}_{\mathrm{b}} \\
& \mathrm{A}\left(\mathrm{u}_{2}, \mathbf{v}_{2}\right)+\mathrm{B}\left(\phi_{2}, \mathbf{v}_{2}\right)=\mathrm{L}_{\mathrm{d}}\left(\mathrm{d}_{1}, \varphi_{1}\right) \\
& \mathrm{B}\left(\varphi_{2}, \mathbf{u}_{2}\right)-\mathrm{C}\left(\phi_{2}, \varphi_{2}\right)=0 \\
& \operatorname{para} \mathbf{u}_{2}, \phi_{2} \in \mathrm{V}_{\mathrm{a}} \mathrm{e} \forall \mathbf{v}_{2}, \forall \varphi_{2} \in \mathrm{V}_{\mathrm{a}} \\
& \int_{\Omega} \rho d \Omega \leq \Omega_{s} \\
& 0 \leq \rho \leq 1
\end{array}
$$

onde: 
$\mathrm{V}_{\mathrm{b}}=\left\{\mathbf{v}=v_{i} \overline{\mathbf{e}}_{i}, \varphi: v_{i}, \varphi \in \mathrm{H}^{1}(\Omega) \operatorname{com} \mathbf{v}=0\right.$ em $\Gamma_{\mathrm{u}}$ e $\varphi=0$ em $\Gamma_{\phi}$ e $\Gamma_{d_{1}} i=1$ ou 3$\}$. Pela alteração no valor da flexibilidade média nos podemos controlar a força de blocagem.

\subsection{Função multi-objetivo e definição do problema de otimização}

Para combinarmos ambos problemas de otimização a seguinte função objetivo é proposta (SILVA; NISHIWAKI; KIKUCHI, 1999):

$$
F=\frac{L_{2}\left(\mathbf{u}_{1}, \phi_{1}\right)}{L_{3}\left(\mathbf{u}_{3}, \phi_{3}\right)}
$$

e mais generalizada na forma:

$$
\begin{aligned}
& F=w^{*} \ln \left(L_{2}\left(\mathbf{u}_{1}, \phi_{1}\right)\right)-(1-w) * \ln \left(L_{3}\left(\mathbf{u}_{3}, \phi_{3}\right)\right) \\
& 0 \leq w \leq 1
\end{aligned}
$$

onde $w$ é um coeficiente de peso. A segunda função objetivo permite controlar a contribuição da transdução média (Eq. (4.21)) e a flexibilidade média (Eq. (4.23)) no projeto, sendo a generalização da Eq. (4.25). Foi observado que qualquer variação na transdução média tem uma pequena influência no projeto do atuador do que a flexibilidade média. Nestes problemas, um grande valor de $w$ deve ser usado para garantir a maximização da transdução média $\mathrm{L}_{2}\left(\mathrm{u}_{1}, \phi_{1}\right)$, e assim o deslocamento gerado. Além disso, esta função permite controlar a flexibilidade final da estrutura, e, portanto a força de blocagem.

O novo problema de otimização (definido na forma contínua) pode ser expresso como (SILVA; NISHIWAKI; KIKUCHI, 1999): 
Maximizar : F

$$
\begin{array}{ll}
\text { tal que } & : \mathbf{t}_{3}=-\mathbf{t}_{2} \quad\left(\Gamma_{\mathrm{t}_{3}}=\Gamma_{\mathrm{t}_{2}}\right) \\
& \mathrm{A}\left(\mathbf{u}_{1}, \mathbf{v}_{1}\right)+\mathrm{B}\left(\phi_{1}, \mathbf{v}_{1}\right)=0 \\
& \mathrm{~B}\left(\varphi_{1}, \mathbf{u}_{1}\right)-\mathrm{C}\left(\phi_{1}, \varphi_{1}\right)=\mathrm{L}_{\mathrm{t}}\left(\mathbf{t}_{1}, \mathbf{v}_{1}\right) \\
& \operatorname{para} \mathbf{u}_{1}, \phi_{1} \in \mathrm{V}_{\mathrm{a}} \mathrm{e} \forall \mathbf{v}_{1}, \forall \varphi_{1} \in \mathrm{V}_{\mathrm{a}} \\
& \mathrm{A}\left(\mathbf{u}_{2}, \mathbf{v}_{2}\right)+\mathrm{B}\left(\phi_{2}, \mathbf{v}_{2}\right)=\mathrm{L}_{\mathrm{d}}\left(\mathrm{d}_{1}, \varphi_{1}\right) \\
& \mathrm{B}\left(\varphi_{2}, \mathbf{u}_{2}\right)-\mathrm{C}\left(\phi_{2}, \varphi_{2}\right)=0 \\
& \operatorname{para} \mathbf{u}_{2}, \phi_{2} \in \mathrm{V}_{\mathrm{a}} \mathrm{e} \forall \mathbf{v}_{2}, \forall \varphi_{2} \in \mathrm{V}_{\mathrm{a}} \\
& \mathrm{A}\left(\mathbf{u}_{3}, \mathbf{v}_{3}\right)+\mathrm{B}\left(\phi_{3}, \mathbf{v}_{3}\right)=\mathrm{L}_{\mathrm{t}}\left(\mathbf{t}_{3}, \mathrm{~V}_{3}\right) \\
& \mathrm{B}\left(\varphi_{3}, \mathbf{u}_{3}\right)-\mathrm{C}\left(\phi_{3}, \varphi_{3}\right)=0 \\
& \operatorname{para} \mathbf{u}_{3}, \phi_{3} \in \mathrm{V}_{\mathrm{b}} \mathrm{e} \forall \mathbf{v}_{3}, \forall \varphi_{3} \in \mathrm{V}_{\mathrm{b}} \\
& \int_{\Omega} \rho d \Omega \leq \Omega \\
& 0 \leq \rho \leq 1
\end{array}
$$

onde $\rho$ (variável de projeto) é a função de pseudo-densidade do material utilizado no projeto, o qual pode variar de "vazio" $(\rho=0)$ a sólido $(\rho=1)$.

Considerando a discussão anterior, a função objetivo é composta de dois casos de cargas mostrados nos casos (a) e (b) da Fig. 4.3. Estes casos de cargas são resolvidos separadamente. O primeiro caso (ver caso (a) da Fig. 4.3) é usado para o cálculo da transdução média e o segundo caso (ver caso (b) da Fig. 4.3) para o cálculo da flexibilidade média. Uma variação prévia da função objetivo pode ser obtida, dependendo das condições essenciais de projeto do atuador piezelétrico flextensional.

Neste estudo, ainda que a formulação presente é geral, o projeto de otimização é conduzido para material elástico no subdomínio $S$, mantendo inalterável o subdomínio do material piezelétrico. Uma mudança no domínio da piezocerâmica não parece ser interessante na prática para aplicação em atuador, devido às características frágeis da piezocerâmica. 


\subsection{Análise de sensibilidades}

Os gradientes (ou derivadas) da função objetivo e restrições são chamados de sensibilidades do problema de otimização. O cálculo desses gradientes é importante devido à necessidade da linearização da função objetivo em relação as variáveis do problema, como veremos mais adiante na implementação numérica da rotina de programação linear seqüencial (PLS). Diante desta necessidade, neste item são desenvolvidos os gradientes da função objetivo, definidos para a otimização topológica do atuador flextensional piezelétrico.

$\mathrm{O}$ gradiente da função $\mathrm{F}$ relativo à variável de projeto $\mathrm{A}_{\mathrm{n}}$ (onde $\mathrm{A}_{\mathrm{n}}$ pode ser também $a_{n}$ e $b_{n}$ ) é dado para a primeira função objetivo por:

$$
\frac{\partial F}{\partial A_{n}}=\frac{1}{L_{3}\left(\mathbf{u}_{3}, \phi_{3}\right)}\left(\frac{\partial L_{2}\left(\mathbf{u}_{1}, \phi_{1}\right)}{\partial A_{n}}\right)-\frac{L_{2}\left(\mathbf{u}_{1}, \phi_{1}\right)}{\left(L_{3}\left(\mathbf{u}_{3}, \phi_{3}\right)\right)^{2}}\left(\frac{\partial L_{3}\left(\mathbf{u}_{3}, \phi_{3}\right)}{\partial A_{n}}\right)
$$

e, para a segunda função objetivo por:

$$
\frac{\partial F}{\partial A_{n}}=\frac{1}{L_{2}\left(\mathbf{u}_{1}, \phi_{1}\right)}\left(\frac{\partial L_{2}\left(\mathbf{u}_{1}, \phi_{1}\right)}{\partial A_{n}}\right)-\frac{(1-w)}{\left(L_{3}\left(\mathbf{u}_{3}, \phi_{3}\right)\right)}\left(\frac{\partial L_{3}\left(\mathbf{u}_{3}, \phi_{3}\right)}{\partial A_{n}}\right)
$$

onde $\partial \mathrm{L}_{2}\left(\mathrm{u}_{1}, \phi_{1}\right) / \partial \mathrm{A}_{\mathrm{n}}$ e $\partial \mathrm{L}_{3}\left(\mathrm{u}_{3}, \phi_{3}\right) / \partial \mathrm{A}_{\mathrm{n}}$ são as derivadas da transdução e flexibilidade média, respectivamente, em relação a variável do problema. $\mathrm{Na}$ seqüência são desenvolvidas as duas derivadas.

Sabemos que a transdução média é dada pela Eq. (4.21), cuja derivada em relação a variável do problema $\mathrm{A}_{\mathrm{n}}$, é dada por: 


$$
\begin{aligned}
\frac{\partial L_{2}\left(\mathbf{u}_{1}, \phi_{1}\right)}{\partial A_{n}} & =A\left(\frac{\partial \mathbf{u}_{2}}{\partial A_{n}}, \mathbf{u}_{1}\right)+\int_{\Omega} \varepsilon\left(\mathbf{u}_{2}\right)^{t} \frac{\partial \mathbf{c}^{E}}{\partial A_{n}}\left(\mathbf{u}_{1}\right) d \Omega+A\left(\mathbf{u}_{2}, \frac{\partial \mathbf{u}_{1}}{\partial A_{n}}\right)+ \\
& +B\left(\frac{\partial \phi_{2}}{\partial A_{n}}, \mathbf{u}_{1}\right)+\int_{\Omega}\left(\nabla \phi_{2}\right)^{t} \frac{\partial \mathbf{e}^{t}}{\partial A_{n}} \varepsilon\left(\mathbf{u}_{1}\right) d \Omega+B\left(\phi_{2}, \frac{\partial \mathbf{u}_{1}}{\partial A_{n}}\right)+ \\
& +B\left(\phi_{1}, \frac{\partial \mathbf{u}_{2}}{\partial A_{n}}\right)+\int_{\Omega} \varepsilon\left(\mathbf{u}_{2}\right)^{t} \frac{\partial \mathbf{e}}{\partial A_{n}} \nabla \phi_{1} d \Omega+B\left(\frac{\partial \phi_{1}}{\partial A_{n}}, \mathbf{u}_{2}\right)+ \\
& -C\left(\frac{\partial \phi_{2}}{\partial A_{n}}, \phi_{1}\right)-\int_{\Omega}\left(\nabla \phi_{2}\right)^{t} \frac{\partial \varepsilon_{D}^{S}}{\partial A_{n}} \nabla \phi_{1} d \Omega-C\left(\phi_{2}, \frac{\partial \phi_{1}}{\partial A_{n}}\right)
\end{aligned}
$$

sabendo-se que (ver Eq.(4.22)):

$$
\frac{\partial L_{2}\left(\mathbf{u}_{1}, \phi_{1}\right)}{\partial A_{n}}=\int_{\Gamma_{t_{2}}} \mathbf{t}_{2} \frac{\partial \mathbf{u}_{1}}{\partial A_{n}} d \Gamma+\int_{\Gamma_{t 2}} d_{2} \frac{\partial \phi_{1}}{\partial A_{n}} d \Gamma
$$

desde que $\mathbf{t}_{2}$ e $\mathbf{d}_{2}$ não dependam das variáveis de projeto. Considerando as equações de equilíbrio (Eq. (4.21)):

$$
\begin{aligned}
& A\left(\mathbf{u}_{2}, \frac{\partial \mathbf{u}_{1}}{\partial A_{n}}\right)+B\left(\frac{\partial \phi_{2}}{\partial A_{n}}, \mathbf{u}_{1}\right)=L_{t}\left(\mathbf{t}_{2}, \frac{\partial \mathbf{u}_{1}}{\partial A_{n}}\right) \\
& B\left(\frac{\partial \phi_{1}}{\partial A_{n}}, \mathbf{u}_{2}\right)-C\left(\phi_{2}, \frac{\partial \phi_{1}}{\partial A_{n}}\right)=L_{d}\left(d_{2}, \frac{\partial \phi_{1}}{\partial A_{n}}\right)
\end{aligned}
$$

então:

$$
A\left(\mathbf{u}_{2}, \frac{\partial \mathbf{u}_{1}}{\partial A_{n}}\right)+B\left(\frac{\partial \phi_{2}}{\partial A_{n}}, \mathbf{u}_{1}\right)+B\left(\frac{\partial \phi_{1}}{\partial A_{n}}, \mathbf{u}_{2}\right)-C\left(\phi_{2}, \frac{\partial \phi_{1}}{\partial A_{n}}\right)=\frac{\partial L_{2}\left(\mathbf{u}_{1}, \phi_{1}\right)}{\partial A_{n}}
$$

analogamente, pode-se escrever: 
$A\left(\mathbf{u}_{1}, \frac{\partial \mathbf{u}_{2}}{\partial A_{n}}\right)+B\left(\frac{\partial \phi_{1}}{\partial A_{n}}, \mathbf{u}_{2}\right)+B\left(\frac{\partial \phi_{2}}{\partial A_{n}}, \mathbf{u}_{1}\right)-C\left(\phi_{1}, \frac{\partial \phi_{2}}{\partial A_{n}}\right)=\frac{\partial L_{1}\left(\mathbf{u}_{2}, \phi_{2}\right)}{\partial A_{n}}=\frac{\partial L_{2}\left(\mathbf{u}_{1}, \phi_{1}\right)}{\partial A_{n}}$

desde que: $L_{1}\left(\mathbf{u}_{2}, \phi_{2}\right)=L_{2}\left(\mathbf{u}_{1}, \phi_{1}\right)$.

Como visto no capítulo $3, \mathbf{c}^{E}$ e $\varepsilon_{d}^{S}$ são tensores simétricos, então:

$$
\begin{aligned}
& A\left(\mathbf{u}_{1}, \frac{\partial \mathbf{u}_{2}}{\partial A_{n}}\right)=A\left(\frac{\partial \mathbf{u}_{2}}{\partial A_{n}}, \mathbf{u}_{1}\right) \\
& C\left(\phi_{1}, \frac{\partial \phi_{2}}{\partial A_{n}}\right)=C\left(\frac{\partial \phi_{2}}{\partial A_{n}}, \phi_{1}\right)
\end{aligned}
$$

Substituindo as Eqs. (4.32) e (4.33) na Eq. (4.29), considerando a Eq. (4.34), obtemos:

$$
\begin{aligned}
\frac{\partial L_{2}\left(\mathbf{u}_{1}, \phi_{1}\right)}{\partial A_{n}}= & \frac{\partial L_{2}\left(\mathbf{u}_{1}, \phi_{1}\right)}{\partial A_{n}}+\frac{\partial L_{2}\left(\mathbf{u}_{1}, \phi_{1}\right)}{\partial A_{n}}+ \\
& +\int_{\Omega} \varepsilon\left(\mathbf{u}_{2}\right)^{t} \frac{\partial \mathbf{c}^{E}}{\partial A_{n}}\left(\mathbf{u}_{1}\right) d \Omega+\int_{\Omega}\left(\nabla \phi_{2}\right)^{t} \frac{\partial \mathbf{e}^{t}}{\partial A_{n}} \mathcal{E}\left(\mathbf{u}_{1}\right) d \Omega+ \\
& +\int_{\Omega} \varepsilon\left(\mathbf{u}_{2}\right)^{t} \frac{\partial \mathbf{e}}{\partial A_{n}} \nabla \phi_{1} d \Omega-\int_{\Omega}\left(\nabla \phi_{2}\right)^{t} \frac{\partial \mathbf{\varepsilon}_{D}^{S}}{\partial A_{n}} \nabla \phi_{1} d \Omega
\end{aligned}
$$

Portanto

$$
\begin{aligned}
\frac{\partial L_{2}\left(\mathbf{u}_{1}, \phi_{1}\right)}{\partial A_{n}}= & -\int_{\Omega} \varepsilon\left(\mathbf{u}_{2}\right)^{t} \frac{\partial \mathbf{c}^{E}}{\partial A_{n}}\left(\mathbf{u}_{1}\right) d \Omega-\int_{\Omega}\left(\nabla \phi_{2}\right)^{t} \frac{\partial \mathbf{e}^{t}}{\partial A_{n}} \mathcal{E}\left(\mathbf{u}_{1}\right) d \Omega+ \\
& -\int_{\Omega} \varepsilon\left(\mathbf{u}_{2}\right)^{t} \frac{\partial \mathbf{e}}{\partial A_{n}} \nabla \phi_{1} d \Omega+\int_{\Omega}\left(\nabla \phi_{2}\right)^{t} \frac{\partial \mathbf{\varepsilon}_{D}^{E}}{\partial A_{n}} \nabla \phi_{1} d \Omega
\end{aligned}
$$


Finalmente, considerando a formulação do MEF, à análise de sensibilidade pode ser expressa na forma matricial:

$$
\frac{\partial L_{2}\left(\mathbf{U}_{1}, \phi_{1}\right)}{\partial A_{n}}=-\left\{\begin{array}{c}
\mathbf{U}_{2}^{t} \\
\phi_{2}^{t}
\end{array}\right\}^{t}\left[\begin{array}{cc}
\frac{\partial \mathbf{K}_{\mathbf{u u}}}{\partial A_{n}} & \frac{\partial \mathbf{K}_{\mathbf{u} \phi}}{\partial A_{n}} \\
\frac{\partial \mathbf{K}_{\mathbf{u} \phi}}{\partial A_{n}} & -\frac{\partial \mathbf{K}_{\phi \phi}}{\partial A_{n}}
\end{array}\right]\left\{\begin{array}{c}
\mathbf{U}_{1} \\
\phi_{1}
\end{array}\right\}
$$

A Eq. (4.37), é uma expressão geral para a análise de sensibilidade, pois considera que todo o domínio $(\Omega)$ discretizado seja otimizável. No entanto, o elemento piezelétrico é não-otimizavel (não depende da variável de projeto). Com isto, a Eq. (4.37), pode ser simplificada:

$$
\frac{\partial L_{2}\left(\mathbf{U}_{1}, \phi_{1}\right)}{\partial A_{n}}=-\left\{\begin{array}{c}
\mathbf{U}_{2}^{t} \\
\phi_{2}^{t}
\end{array}\right\}^{t}\left[\begin{array}{cc}
\frac{\partial \mathbf{K}_{\mathbf{u u}}}{\partial A_{n}} & 0 \\
0 & 0
\end{array}\right]\left\{\begin{array}{c}
\mathbf{U}_{1} \\
\phi_{1}
\end{array}\right\}=-\mathbf{U}_{2}^{t} \frac{\partial \mathbf{K}_{\mathbf{u u}}}{\partial A_{n}} \mathbf{U}_{1}
$$

lembrando, que para o elemento piezelétrico $\frac{\partial \mathbf{K}_{\mathrm{uu}}}{\partial A_{n}}$ é igual a zero.

A sensibilidade da flexibilidade média é obtida considerando $\mathbf{u}_{1}=\mathbf{u}_{2}=\mathbf{u}_{3}$ e $\phi_{1}=\phi_{2}=\phi_{3}$ :

$$
\begin{aligned}
\frac{\partial L_{3}\left(\mathbf{u}_{3}, \phi_{3}\right)}{\partial A_{n}}= & -\int_{\Omega} \varepsilon\left(\mathbf{u}_{3}\right)^{t} \frac{\partial \mathbf{c}^{E}}{\partial A_{n}}\left(\mathbf{u}_{3}\right) d \Omega-\int_{\Omega}\left(\nabla \phi_{3}\right)^{t} \frac{\partial \mathbf{e}^{t}}{\partial A_{n}} \mathcal{E}\left(\mathbf{u}_{3}\right) d \Omega+ \\
& -\int_{\Omega} \varepsilon\left(\mathbf{u}_{3}\right)^{t} \frac{\partial \mathbf{e}}{\partial A_{n}} \nabla \phi_{3} d \Omega+\int_{\Omega}\left(\nabla \phi_{3}\right)^{t} \frac{\partial \mathbf{\varepsilon}_{D}^{S}}{\partial A_{n}} \nabla \phi_{3} d \Omega
\end{aligned}
$$

e, considerando a formulação do MEF, à análise de sensibilidade pode ser expressa na forma matricial: 


$$
\frac{\partial L_{3}\left(\mathbf{U}_{3}, \phi_{3}\right)}{\partial A_{n}}=-\left\{\begin{array}{l}
\mathbf{U}_{3}^{t} \\
\phi_{3}^{t}
\end{array}\right\}^{t}\left[\begin{array}{cc}
\frac{\partial \mathbf{K}_{\mathbf{u u}}}{\partial A_{n}} & 0 \\
0 & 0
\end{array}\right]\left\{\begin{array}{c}
\mathbf{U}_{3} \\
\phi_{3}
\end{array}\right\}=-\mathbf{U}_{3}^{t} \frac{\partial \mathbf{K}_{\mathbf{u u}}}{\partial A_{n}} \mathbf{U}_{3}
$$




\section{Capítulo 5: Implementação Numérica}

\subsection{Introdução}

O problema de otimização, apresentado no capítulo 4, pode ser resolvido numericamente através de um algoritmo computacional que combina o método de otimização de programação linear (PLS) (DANTZIG, 1963; YANG; CHUANG, 1994 e HAFTKA; GURDAL; KAMAT, 1996) com o método numérico de análise por elementos finitos (MEF).

Pode-se resolver os problemas de otimização estrutural, utilizando diversas aproximações distintas, como, por exemplo, o critério de optimalidade (CO) (HASSANI; HINTON, 1998c), o método de PLS, o movimento assintótico (MMA) (SVANBERG, 1987) e o método de programação quadrática seqüencial (PQS). O CO é um método de OT específico, no qual, é desenvolvida uma formulação específica para cada problema de otimização que é elaborado. No entanto, o método do $\mathrm{CO}$ é eficiente computacionalmente e foi aplicado em vários problemas de otimização topológica, entre eles o problema de minimização de flexibilidade média (ou maximização de rigidez) (SUZUKI; KIKUCHI, 1991), no problema de otimização de estruturas discretas (tipo treliça) (ROZVANY; ZHOU, 1991; ZHOU; ROZVANY, 1991) e o problema de maximização da freqüência de ressonância (MA; KIKUCHI; CHENG, 1995) e recentemente no projeto de mecanismos flexíveis (ANANTHASURESH; KOTA; GIANCHANDANI, 1994; DE LIMA; SILVA, 2002).

Os métodos de PLS e PQS são métodos classificados como genéricos, ou seja, os métodos genéricos consistem em métodos de otimização que são baseados na chamada teoria de programação matemática e podem ser aplicados em qualquer problema de otimização, seja no projeto de estruturas ou em outros ramos de aplicação. Entre eles, o PLS vem sendo aplicado com sucesso na otimização 
topológica, em particular no projeto de atuadores piezelétricos flextensionais (SILVA; NISHIWAKI; KIKUCHI, 1999 e 2000). O PLS é uma rotina que resolve, seqüencialmente vários subproblemas lineares na busca da solução do problema não linear, o qual geralmente possui uma função objetivo complexa, um grande número de variáveis de projeto e várias restrições. No próximo item é feito um detalhamento maior sobre o PLS.

\subsection{Programação linear seqüencial (PLS)}

O problema de otimização de atuadores piezelétricos flextensionais, considerando as restrições, foi formulado no item 4.4.

A programação linear (YANG; CHUANG, 1994) resolve um problema de otimização em que a função objetivo e as restrições são funções lineares em relação às variáveis do problema, ou seja, o problema deve ter a seguinte forma:

$$
\begin{aligned}
& \underset{\mathbf{x}}{\operatorname{Maximizar}:} F(x)=a_{1} x_{1}+a_{2} x_{2}+\ldots+a_{n} x_{n} \\
& \text { Sujeita à: } \quad g_{i}^{1}(x)=b_{1} x_{1}+b_{2} x_{2}+\ldots+b_{n} x_{n} \leq c_{i},\left(\mathrm{i}=1, \ldots, \mathrm{m}_{1}\right) \\
& g_{j}^{2}(x)=c_{1} x_{1}+c_{2} x_{2}+\ldots+c_{n} x_{n} \geq d_{j},\left(\mathrm{j}=1, \ldots, \mathrm{m}_{2}\right) \\
& g_{k}^{3}(x)=d_{1} x_{1}+d_{2} x_{2}+\ldots+d_{n} x_{n}=e_{k},\left(\mathrm{k}=1, \ldots, \mathrm{m}_{3}\right)
\end{aligned}
$$

Embora, a função objetivo do problema de otimização mostrado no capítulo 4 (item 4.4), que queremos maximizar, não seja linear em relação às variáveis do problema, que são as densidades de cada ponto do domínio estendido fixo de projeto, o problema pode ser linearizado calculando-se a Série de Taylor de $1^{\mathrm{a}}$ ordem da função objetivo. Sabe-se do cálculo diferencial que a Série de Taylor pode representar uma função $f(x)$, para $x$ próximo de um valor $x_{0}$, a menos de um erro $e(x)$, como ilustra a equação abaixo: 


$$
f(x)=f\left(x_{0}\right)+\frac{\partial f}{\partial x}\left(x-x_{0}\right)+\frac{\partial^{2} f}{\partial x^{2}} \frac{\left(x-x_{0}\right)^{2}}{2 !}+\ldots+\frac{\partial^{N} f}{\partial x^{N}} \frac{\left(x-x_{0}\right)^{N}}{N !}+e(x)
$$

Para uma função com $n$ variáveis, teríamos:

$$
\begin{aligned}
& f\left(x_{1}, x_{2}, \ldots, x_{n}\right)=f\left(x_{0}\right)+\frac{\partial f}{\partial x_{1}}\left(x_{1}-x_{01}\right)+\ldots+\frac{\partial^{N} f}{\partial x_{1}^{N}} \frac{\left(x_{1}-x_{01}\right)^{N}}{N !}+
\end{aligned}
$$

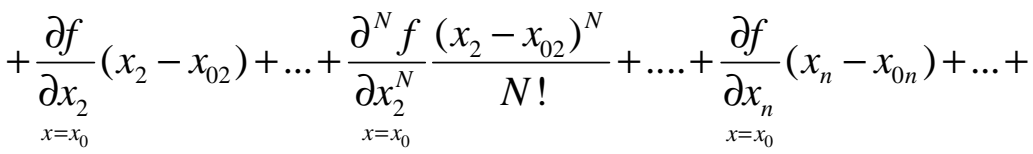

$$
\begin{aligned}
& +\frac{\partial^{N} f}{\partial x_{n}^{N}} \frac{\left(x_{n}-x_{0 n}\right)^{N}}{N !}+e\left(x_{1}, x_{2}, \ldots, x_{n}\right)
\end{aligned}
$$

Os termos com derivada de segunda ordem ou superior são desprezados, obtendo uma linearização da função objetivo, para ser utilizada na PL. No entanto, também há métodos que realizam otimização de funções considerando aproximação com termos de derivada de segunda ordem ou superior, como por exemplo, a Programação Quadrática Seqüencial (PQS).

Então, podemos linearizar a função objetivo $\left(\mathrm{F}_{2}\right)$, como:

$$
F_{2}=F_{2}^{0}+\frac{\partial F_{2}}{\partial \rho_{1}}\left(\rho_{1}-\rho_{1}^{0}\right)+\frac{\partial F_{2}}{\partial \rho_{2}}\left(\rho_{2}-\rho_{2}^{0}\right)+\ldots+\frac{\partial F_{2}}{\partial \rho_{n}}\left(\rho_{n}-\rho_{n}^{0}\right)
$$

Separando os termos constantes dos termos dependentes das variáveis (Eq (5.7), temos: 


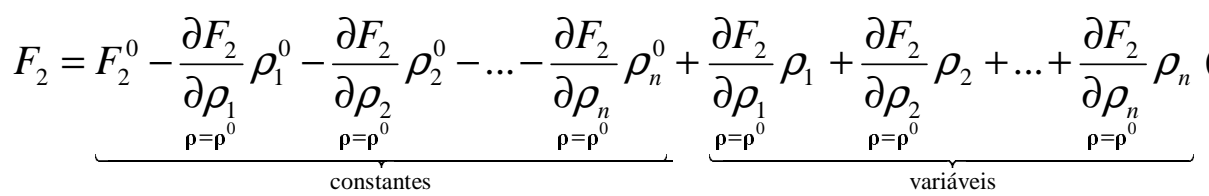

As constantes podem ser retiradas da Eq. (5.8), pois não influenciam no processo de maximização da função. Desta maneira a função a ser maximizada na PL é dada por:

$$
F_{2}^{\text {linear }}=\frac{\partial F_{2}}{\partial \rho_{1}} \rho_{1}+\frac{\partial F_{2}}{\partial \rho_{2}} \rho_{2}+\ldots+\frac{\partial F_{2}}{\partial \rho_{n}} \rho_{n}
$$

Observe que agora a função a ser maximizada (Eq. (5.9)) é linear, onde o coeficiente das densidades $\rho_{i}$ (variáveis) é a derivada (ou sensibilidade) da função objetivo do problema de otimização (função $F_{2}$ ) em relação à variável do problema, no ponto $\boldsymbol{\rho}=\boldsymbol{\rho}^{0}$. Essa derivada foi desenvolvida no capítulo 4 (item 4.5 ).

Como visto anteriormente, a idéia primária do PLS para solucionar o problema de otimização não-linear é fazer uma aproximação por sucessivos subproblemas lineares que são resolvidos por método de programação linear, Simplex (DANTZIG, 1963) ou Kamarkar (HAFTKA; GURDAL; KAMAT, 1996). Para que essa aproximação seja válida é necessário limitar a variação do valor da variável de projeto (variável do problema de otimização) em cada subproblema linear através dos limites móveis (THOMAS; VANDERPLAATS; SHYY, 1992). Para entender melhor este conceito, considere a função não-linear $\mathrm{F}(\mathrm{x})$ mostrada no gráfico da Fig. 5.1. 


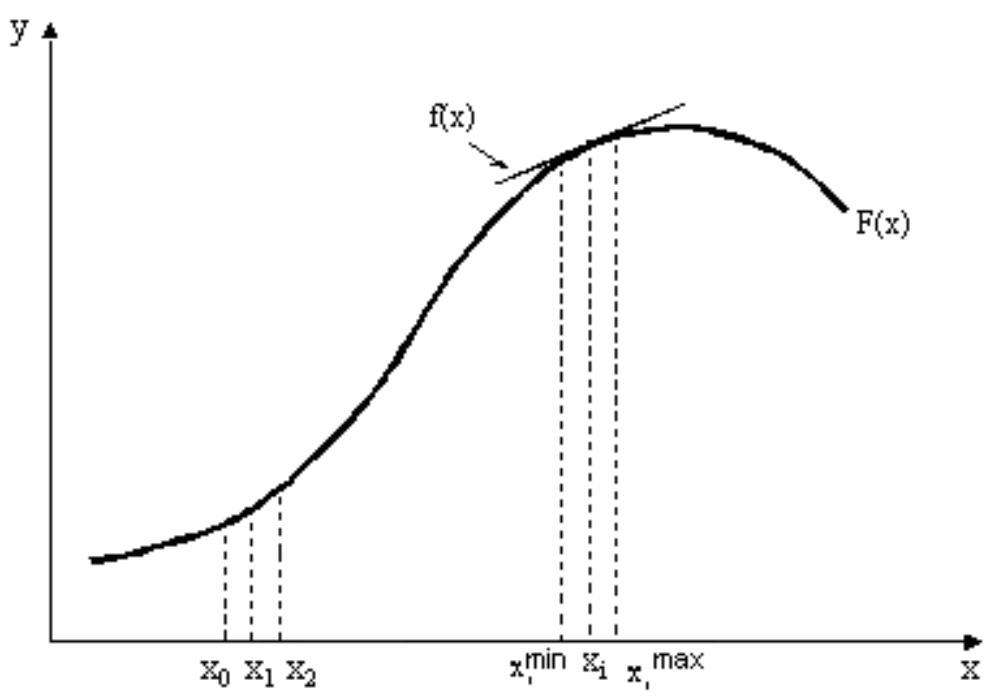

Figura 5.1 - Limites móveis.

Considere também a função linear $f(x)$ como uma aproximação da função não linear $F(x)$ em torno do ponto $x_{i}$. Observa-se que a aproximação linear (função $f(x))$ somente é válida em torno do ponto $x_{i}$, assim, à medida que se afasta de $x_{i}$ se distancia do valor real da função $F(x)$. Conseqüentemente, defini-se os valores máximo e mínimo em torno de $x_{i}$, onde a aproximação linear é válida, os limites móveis da variável $x_{i}$. A cada iteração a rotina de PLS maximiza a função linear $f(x)$ no intervalo dos limites móveis $\left(x_{\mathrm{i}}^{\min }\right.$ e $x_{\mathrm{i}}^{\max }$ ) e encontra o valor ótimo para a variável $x_{i}$, que será utilizada na iteração seguinte. Assim as iterações seguem sucessivamente, substituindo-se como valor inicial da iteração posterior o valor ótimo de $x_{i}$ encontrado na iteração anterior, até ocorrer a convergência da solução.

Os limites são considerados móveis pelo fato da variável $x_{i}$ assumir um valor diferente a cada iteração da rotina de PLS. Esses limites são definidos como valores relativos em relação ao valor dessa variável. Porém é necessário observar que, se os valores utilizados para os limites móveis forem muito grandes, o erro da aproximação linear será grande, podendo causar inclusive a perda do ponto ótimo. Em contra partida, se forem muito pequenos o custo computacional para obter a solução ótima (no caso o ponto de máximo da função $F(x)$ ) será muito grande. Uma forma de tentar minimizar esse impasse e tornar mais rápido a convergência da 
solução é assumir valores grandes para os limites móveis na região em que a função não linear $F(x)$ apresenta um valor baixo de derivadas (região do ponto $x_{0}$ mostrada na Fig. 4.1), pois nessa região a função não linear $F(x)$ tem praticamente o mesmo comportamento da aproximação linear (função $f(x)$ ). Já na região em que a função apresenta grandes derivadas os limites móveis devem ser pequenos. Além disso, à medida que nos aproximamos da convergência da solução (ponto de máximo da função não linear $F(x)$ ) devemos reduzir bastante os valores dos limites móveis, caso contrário podemos "passar" pelo ponto máximo gerando oscilação no valor da função $F(x)$.

Uma escolha inadequada dos valores dos limites móveis pode tornar o método da PLS desvantajoso, bem como não fazer um ajuste mais preciso desses limites quando nos aproximamos do valor ótimo da função objetivo. Em geral, são utilizados valores em torno de $15 \%$ do valor de $x_{i}$ para os limites móveis. Uma alternativa é utilizar as informações das derivadas da função objetivo e das restrições para a determinação dos limites móveis (CHEN, 1993) (não considerada neste trabalho).

Além da função objetivo, o PL exige que as restrições do problema de otimização também sejam funções lineares em relação às variáveis de problema. Considerando em particular o problema de otimização definido neste trabalho (item 4.4), as restrições que dependem das variáveis do problema são dadas por:

$$
\begin{aligned}
& \int_{\Omega} \rho d \Omega-\Omega_{s} \leq 0 \\
& 0 \leq \rho \leq 1
\end{aligned}
$$

onde a Eq. (5.10) é a restrição de volume de material e a Eq. (5.11) é a restrição ao valor limite (superior e inferior) da variável de problema.

A Eq. (5.10) é composta por duas parcelas, sendo uma delas indicada por $\Omega_{s}$ que é um valor desejado e estabelecido previamente para o volume final de material na estrutura a ser otimizada. A outra parcela, indicada pela integral $\int_{\Omega} \rho d \Omega$, 
é o valor do volume de material obtido após a otimização e que depende dos valores da densidade de cada ponto do domínio da estrutura otimizada. Como o domínio da estrutura é discretizado em elementos finitos, para se obter uma solução numérica do problema de otimização, consideramos a densidade de cada elemento $\left(\rho_{i}\right)$ como sendo a variável do problema, de tal forma que o volume total de material na estrutura possa ser representado pela somatória do produto entre o volume de cada elemento e sua densidade. Na realidade a variável $\rho_{i}$, apesar de ser chamada de densidade, é na verdade um valor relativo e não deve confundida com o peso específico. Desta forma, temos a restrição da Eq. (5.11) como uma função linear em relação a variável do problema $\rho_{i}$, ou seja:

$$
\sum_{i=1}^{M} V_{i} \rho_{i} \leq V^{*}
$$

onde $V_{i}$ é o volume de cada elemento e $V^{*}$ é a restrição do volume de material no domínio discretizado e $M$ é o número total de elementos finitos.

Já a Eq. (5.11) (restrição da variável do problema), faz com que o valor da variável do problema seja limitado dentro de uma faixa. Esta faixa representa uma variação da presença de material no domínio da estrutura, onde o valor 0 (zero) indica ausência de material ("buraco") e 1 indica a total presença de material. Observa-se que, como a rigidez do elemento finito está condicionada diretamente com as propriedades efetivas do material (capítulo 3), a penalidade do material intermediário (capítulo 2) é feita na matriz de rigidez $\boldsymbol{k}_{\boldsymbol{e}}$ do elemento, da seguinte forma:

$$
\mathbf{k}_{\mathrm{e}}=\rho_{\mathrm{e}}^{p} \mathbf{k}_{\mathrm{e}}^{0}
$$

onde $\rho_{\mathrm{e}}$ é a densidade do elemento, $p$ é o fator de penalidade e $\mathbf{k}_{\mathrm{e}}^{0}$ é a matriz de rigidez base do elemento (considerando o elemento inteiramente sólido). Como a 
matriz de rigidez global $\boldsymbol{K}$ é montada a partir da matriz $\boldsymbol{k}_{\boldsymbol{e}}$ de cada elemento, é possível que alguns pontos da diagonal da matriz $\boldsymbol{K}$ sejam nulos, caso seja considerado algum valor de densidade $\left(\rho_{i}\right)$ igual a zero, o que acarretaria em divisão por zero durante as operações realizadas com essa matriz. Portanto, o valor limite inferior da variável do problema de otimização não pode ser considerado exatamente zero, e sim um valor muito pequeno (próximo de zero), isto é necessário para evitar singularidades na matriz de rigidez global $\boldsymbol{K}$ e conseqüentemente o maucondicionamento numérico.

Finalmente, o problema a ser inserido na rotina de Programação Linear adquire a seguinte forma:

$$
\begin{aligned}
& \underset{\mathbf{p}}{\operatorname{Maximizar}:} F_{2}^{\text {linear }}=\frac{\partial F_{2}}{\partial \rho_{1}} \rho_{1}+\frac{\partial F_{2}}{\partial \rho_{2}} \rho_{2}+\ldots+\frac{\partial F_{2}}{\partial \rho_{n}} \rho_{n} \\
& \text { Sujeita à: } \quad \sum_{\substack{\rho^{0}=\mathbf{p}^{0} \\
\mathbf{p}=\mathbf{p}^{0}}}^{M} V_{i} \rho_{i} \leq V^{*} \\
& 0 \leq \rho_{i}^{\min } \leq \rho_{i} \leq \rho_{i}^{\max }, \cdot \mathrm{i}=1, \ldots, \mathrm{M}
\end{aligned}
$$

A solução numérica do problema de programação linear (PL) pode ser obtida usando-se o método Simplex (DANTZIG, 1963) ou o método Kamarkar (HAFTKA; GURDAL; KAMAT, 1996) que buscam, dentro de um conjunto de soluções possíveis para o problema, à solução que satisfaz as restrições e maximize a função objetivo. Geralmente, as rotinas de PL podem ser encontradas na literatura, como, por exemplo, a rotina SIMPLX (PRESS et al., 1999) que usa o método Simplex, ou disponibilizadas gratuitamente pela rede mundial de computadores (internet), como é o caso da rotina conhecida por DSPLP que usa o método de Kamarkar. 


\subsection{Filtro para eliminação da "instabilidade xadrez"}

Como foi exposto no capítulo 2 (item 2.5), a "instabilidade xadrez" (ou “checkerboard") é caracterizado pela instabilidade de presença e ausência de material em certas regiões da topologia obtida MOT, que é causada pela maior rigidez do arranjo da instabilidade xadrez em relação a um arranjo uniforme, o que faz com que o MOT opte por essa configuração.

Constatou-se que a solução para evitar a formação da "instabilidade xadrez", nos resultados da Otimização Topológica, é aumentar a ordem do elemento ou utilizar um filtro que controle a variação das densidades (variáveis de projeto). No entanto, aumentar a ordem do elemento significa trabalhar com elementos finitos com um número grande de nós (maior que 4), o que implica numa solução que produz alto custo computacional devido ao processamento da matriz de rigidez do modelo de MEF. Assim, a solução adotada neste trabalho para evitar a formação da “instabilidade xadrez" foi à utilização de um filtro espacial proposto por Cardoso; Fonseca (1999). Esse filtro suaviza a distribuição espacial das variáveis de projeto ao longo do domínio estendido fixo, através de uma transformação matemática a cada variável do problema de otimização. Alguns trabalhos da literatura sugerem o controle dos gradientes, ou seja, impedindo variações bruscas da variável de projeto no domínio, garantindo o fechamento do espaço de solução, desenvolvido por Bendsøe, 1995, e utilizado por Petersson; Sigmund, 1998 e Cardoso; Fonseca, 1999. Outros trabalhos da literatura sugerem a aplicação do filtro sobre o campo de densidades (SIGMUND, 2000), mas como essas densidades geralmente são as variáveis, atualizadas a cada iteração durante o processo de otimização, ao aplicarmos esse filtro modificamos os valores ótimos encontrados para essas variáveis, "bagunçando" um pouco a otimização realizada. Assim, o filtro espacial com raio de abrangência variável, adotado neste trabalho, é aplicado sobre os limites móveis das variáveis do problema de otimização linearizado (CARDOSO; FONSECA, 1999), ou seja, o problema resolvido por programação linear (veja item $5.2)$.

A aplicação do filtro espacial (CARDOSO; FONSECA, 1999) sobre os 
limites móveis da variável permite um controle simplificado da complexidade da topologia ótima encontrada no processo de Otimização Topológica. Esse filtro é uma variação espacial do filtro originalmente proposto por Swan; Kosaka (1997), que propuseram um esquema de filtragem de vizinhança fixa, em que a densidade de cada elemento depende das densidades dos elementos vizinhos, da seguinte maneira:

$$
\rho_{i}=\frac{\rho_{i} V_{i}+w_{1}^{F} \sum_{j} \rho_{j} V_{j}+w_{2}^{F} \sum_{j} \rho_{j} V_{j}}{V_{i}+w_{1}^{F} \sum_{j} V_{j}+w_{2}^{F} \sum_{j} V_{j}}
$$

onde $V_{i}$ é o volume do elemento $i$ no problema discreto, $w_{1}^{F}$ e $w_{2}^{F}$ são pesos atribuídos heuristicamente aos vizinhos de arestas e vizinhos de vértices, respectivamente.

Em filtros de vizinhança fixa somente elementos vizinhos de aresta e/ou vértice são considerados, o que torna o resultado da otimização dependente da discretização do domínio e restringe a utilização de malhas não regulares. Já nos filtros espaciais são considerados os elementos que estão dentro de um determinado raio de abrangência em torno do elemento central, conforme mostrado na Fig. 5.2.

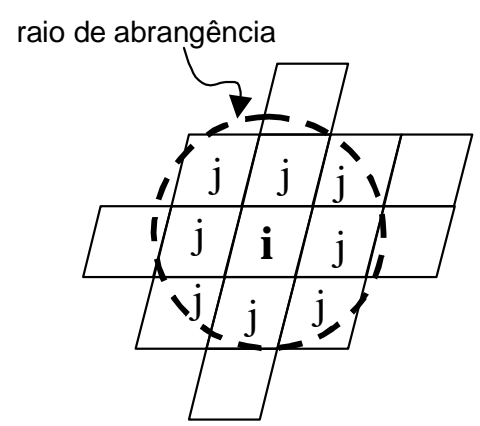

Figura 5.2 - Raio de abrangência nos filtros espaciais.

Os filtros espaciais minimizam a dependência do resultado final ao refino da 
malha de elementos finitos e permitem um controle da complexidade da topologia, ou seja, uma abrangência maior de elementos em torno do elemento central permite obter distribuições mais suaves da variável do problema e, portanto, topologias mais simples. O filtro espacial linear é o mais simples, nele são considerados os elementos vizinhos que se encontram dentro de um raio $R_{\max }$ em torno do elemento central e cujos pesos da média ponderada são calculados, conforme equação abaixo:

$$
W_{i}^{F}=R_{\max }-R_{i j}
$$

onde $R_{i j}$ é a distância entre centróides (menor que $R_{\max }$ ) do elemento central $i$ e do elemento vizinho $j$.

Baseado no conceito do filtro espacial linear, Cardoso; Fonseca (1999) propuseram a seguinte alteração para tornar o filtro de Swan; Kosaka (1997) um filtro espacial:

$$
\rho_{i}=\frac{\rho_{i} V_{i}+\overline{w^{F}} \sum_{j=1}^{n v} \rho_{j} V_{j}}{V_{i}+\overline{w^{F}} \sum_{j=1}^{n v} V_{j}}
$$

com

$$
\overline{w^{F}}=\frac{\sum_{j=1}^{n v} w_{j}^{F}}{n v}
$$

onde:

$$
w_{i}^{F}=\frac{R_{\max }-R_{i j}}{R_{\max }}
$$


então:

$$
w_{i}^{F}=\frac{W_{i}^{F}}{R_{\max }}
$$

e $n v$ é o número de vizinhos determinados por uma varredura fixa em torno do elemento central $i$, sendo $j=1,2, \ldots, n v$. Percebe-se que, desta maneira os pesos fixos para vizinhos de aresta e de vértice não são mais considerados. O filtro proposto por Cardoso; Fonseca (1999) combina as vantagens do filtro de vizinhança fixa, com os benefícios dos filtros espaciais, e como é aplicado diretamente sobre os limites móveis da programação linear, permite obter um controle sobre o gradiente da variável do problema sem impor uma mudança artificial sobre a distribuição das densidades, obtidas da otimização, no domínio de projeto. Ou seja, uma vez calculados os limites móveis para cada variável de projeto, aplica-se a filtragem sobre cada um dos limites, considerando os limites móveis dos vizinhos definidos. Obtém-se, assim, uma distribuição de limites móveis que impede o aparecimento da instabilidade xadrez. A desvantagem da aplicação do filtro sobre os limites móveis é a dependência do desempenho do filtro com métodos de determinação dos limites móveis. Se esses métodos permitirem uma variação muito brusca dos valores dos limites móveis, poderão causar uma atenuação do efeito do filtro. Porém, isso não se configura num grande problema, pois as variações dos limites móveis são limitadas a pequenos valores devido à aproximação linear realizada na programação linear do problema de otimização.

\subsection{Procedimento para implementação do MOT}

Neste item, é apresentado os passos da implementação numérica da solução do problema de otimização apresentado no capítulo 4, onde é implementado um software que resolve o problema através de rotinas de MEF e PL. As rotinas do software são escritas usando a linguagem C. A Fig. 5.3 descreve o fluxo do software de otimização topológica. 
Inicialmente, o software precisa receber a informação (dados iniciais) sobre a geometria, pontos de fixação e aplicação das cargas do domínio inicial (estendido fixo de projeto). Isto é feito através da leitura, pelo software, de um arquivo texto que contém as matrizes de coordenadas, fixação dos graus de liberdade e aplicação de forças mecânicas e elétricas de cada nó da malha de elementos finitos, gerada para o domínio inicial. A malha de elementos finitos é gerada no software comercial de elementos finitos ANSYS.

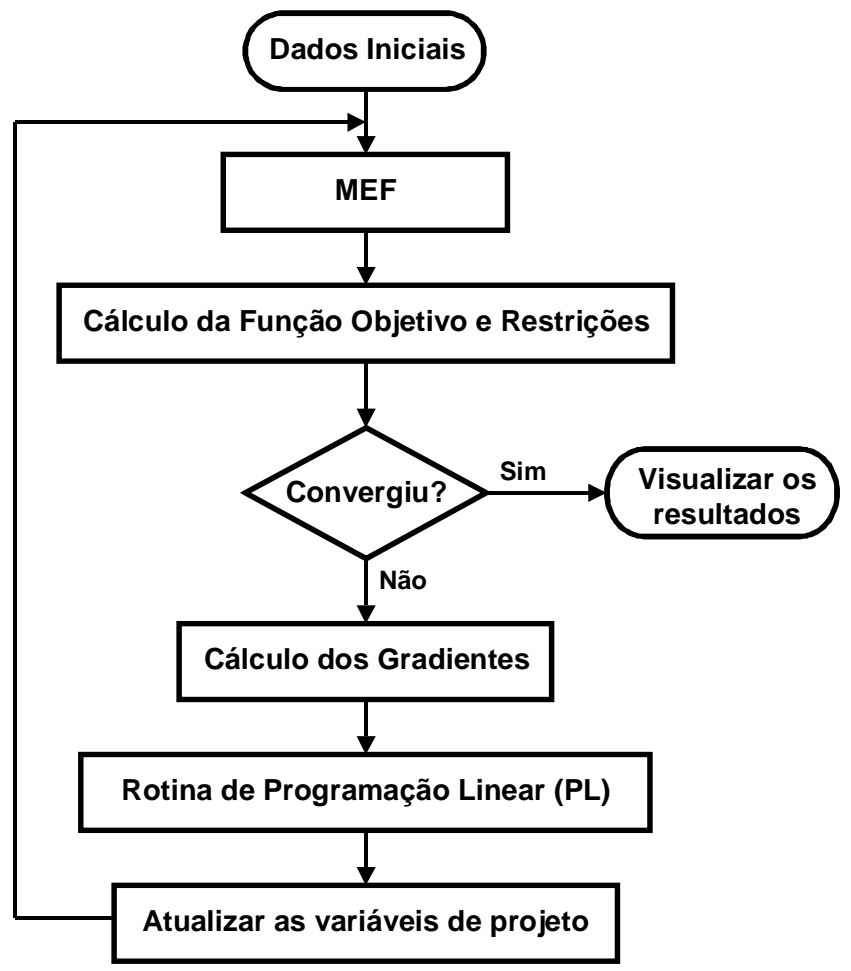

Figura 5.3 - Fluxo do processo iterativo de otimização.

Para implementar numericamente a função multi-objetivo do problema de otimização (item 5.2) para o projeto de atuadores piezelétricos flextensionais, o usuário deve elaborar três situações de carregamentos distintos, para a mesma malha gerada. A Fig. 5.4 ilustra essas situações de carregamento, a partir dos quais serão calculadas a transdução média e a flexibilidade média, e conseqüentemente a função multi-objetivo. Desta forma, a Fig. 5.4(a) mostra a aplicação de cargas elétricas nos 
nós que representam os eletrodos da piezocerâmica. A Fig. 5.4(b) mostra a aplicação da força fictícia no ponto e na direção do deslocamento desejado. Finalmente, a Fig. 5.4(c) mostra que são aterrados todos os nós pertencentes aos eletrodos da piezocerâmica e aplica-se uma força fictícia de sentido oposto da Fig. 5.4(b).
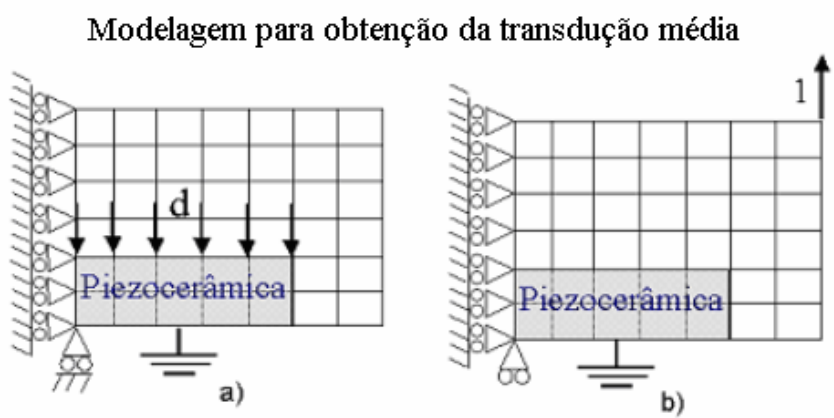

Modelagem para obtenção da flexibilidade média

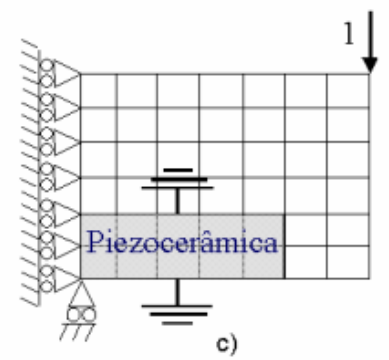

Figura 5.4 - Situações de carregamento para o projeto do atuador piezelétrico flextensional.

O software recebe essas informações e através de uma rotina do MEF são calculados os deslocamentos nodais para cada caso de carga, considerando a mesma malha de elementos finitos. Em seguida, conhecidos os deslocamentos nodais e a matriz de rigidez global (montada pela rotina de MEF), é calculado a transdução média e a flexibilidade média. Desta maneira, é possível calcular também o valor da função objetivo. A forma matricial obtida da formulação contínua é utilizada pelo software para o cálculo da transdução média, flexibilidade média e função objetivo (ver capítulo 4). 
Através da PLS, resolve-se o problema de otimização, fornecendo os gradientes da função objetivo, através de uma rotina que monta o vetor da derivada da função objetivo em relação à densidade (variável do problema) de cada elemento do domínio. Esse vetor representa os coeficientes da função objetivo linearizada para a PL. A rotina de PL exige também a informação sobre as restrições do problema, e essas informações estão contidas em outros vetores montados pelas diversas rotinas do software.

A rotina de programação linear usa o método Kamarkar (HAFTKA; GURDAL; KAMAT, 1996), que recebe esses dados e, através de um processo iterativo, obtém os valores ótimos das variáveis do problema que maximiza a função objetivo para o subproblema. Lembrar que, no presente trabalho, as variáveis de projeto do problema de otimização são as pseudo-densidades relativas $(\rho) \operatorname{dos}$ elementos da malha de elementos finitos, gerada para o domínio inicial. Na primeira iteração, o usuário informa à rotina de PL quaisquer valores para as variáveis do problema, os quais servem de ponto de partida para o processo de busca dos valores ótimos das variáveis. O software utiliza automaticamente o último valor ótimo obtido como valor inicial para as iterações seguintes.

A cada iteração, a matriz de rigidez global $\boldsymbol{K}$, e os cálculos do valor da função objetivo e sua derivada são refeitos, pois os valores ótimos das variáveis do problema são modificados, ou seja, a cada iteração o algoritmo se desloca seqüencialmente na função objetivo e restrições, em pequenos trechos lineares limitados por limites móveis aplicados às variáveis do problema. Após algumas iterações, o processo pela busca do máximo da função objetivo é terminado quando há convergência das iterações, que pode ser verificada quando o valor da função objetivo não apresenta mais variações. Em benefício da rápida convergência das iterações, os limites móveis podem ser alterados, conforme o processo iterativo se aproxima do valor ótimo da função, como já comentado no item 5.2. Inicialmente, atribui-se um limite móvel de $15 \%$ superior e inferior ao valor da pseudodensidade de cada elemento. 


\section{Capítulo 6: Resultados}

\subsection{Introdução}

Neste capítulo são apresentados vários exemplos que ilustram o projeto de atuadores piezelétricos flextensionais usando o método de otimização topológica. Os resultados mostram as dificuldades e a potencilialidade do software no projeto de um atuador, ilustrando todos os parâmetros e outros fatores que influenciam no projeto. $\mathrm{Na}$ primeira fase (item 6.2) do APFLEX (software de otimização topológica desenvolvido neste trabalho) foi possível obter topologias ótimas bem definidas, manipular domínios iniciais com discretização em torno de 1000 elementos, trabalhar com elementos finitos irregulares e evitar a formação da "instabilidade xadrez". Características como formação e eliminação da "instabilidade xadrez", não unicidade da solução, redução das "escalas de cinza", influência do valor inicial de densidades para as densidades e interpretação dos resultados obtidos serão ilustradas e analisadas neste capítulo. Além disso, mostraremos alguns exemplos ilustrando os parâmetros da formulação do problema aplicados ao projeto de atuadores piezelétricos, com o objetivo de verificar o comportamento do atuador segundo a variações destes parâmetros, tais como o coeficiente de atribuição de peso $w$, a restrição de volume de material $\left(V^{*}\right)$, a discretização do domínio inicial de projeto e o fator $p$ de penalidade das densidades intermediárias. Desta forma, será possível comparar os resultados obtidos através destes exemplos com os obtidos da literatura. Na segunda fase do APFLEX é possível trabalhar com domínios discretizados acima de 2500 elementos finitos. Nesta fase, o objetivo é mostrar a potencialidade do software para aplicação em projetos dos mais variados tipos de atuadores piezelétricos flextensionais.

Inicialmente, a programação do software APFLEX foi desenvolvida em ambiente WINDOWS, usando a rotina SIMPLX para resolver a programação linear de otimização das variáveis de projeto. Com o aumento do número de variáveis, surgiu a necessidade de implementar uma rotina robusta e eficiente como a DSPLP. 
Com isto, a rotina DSPLP, escrito na linguagem FORTRAN foi compilada juntamente com o APFLEX, escrito na linguagem C. No campo da programação, a interface entre as linguagens C e Fortran é totalmente possível, para isso devemos fazer a ligação dos arquivos .obj gerados pelos compiladores de cada uma das linguagens ( $\mathrm{C}$ e Fortran). Após adaptado perfeitamente a rotina DSPLP no código fonte do APFLEX e trabalhando em ambiente LINUX, passamos a obter resultados de topologias ótimas a partir de malhas de elementos finitos muito mais refinadas que anteriormente, ou seja, o software agora pode processar com um domínio discretizado em milhares de elementos finitos e com tempo de processamento muito menor ( 10 vezes mais rápido do que usando a rotina SIMPLX no ambiente WINDOWS, para o mesmo problema).

Os modelos de atuadores piezelétricos foram selecionados da literatura. A escala das dimensões dessas configurações geométricas é da ordem de milímetros $(\mathrm{mm})$, sendo que a espessura é unitária. As propriedades usadas para o material piezelétrico (cerâmica piezelétrica) e para a estrutura flexível acoplada são ilustrados na Tabela 6.1. Os atuadores ilustrados a seguir são projetados para deslocar o ponto $B$ (representado no domínio inicial), fornecendo o deslocamento desejado, quando a carga elétrica for aplicada nos eletrodos da piezocerâmica e ser capaz de atuar mediante a aplicação contínua do potencial elétrico. $\mathrm{O}$ fator de penalidade $p$ para todos os resultados apresentados neste trabalho é igual à 3 , e a intensidade de carga elétrica aplicada nos eletrodos é de $1 \mu \mathrm{C} / \mathrm{m}^{2}$ e a força fictícia de intensidade igual à 1 na direção e localização especificada pelo ponto $B$ (mesma direção do deslocamento desejado). Outros valores podem ser aplicados uma vez que o problema de análise é linear. É considerada em todos os resultados a formulação do MEF para elementos finitos isoparamétricos quadriláteros de 4 nós, baseado no estado plano de tensão. Demais informações encontram-se descritas na introdução local de cada exemplo e caso ocorra algum resultado obtido com valores diferentes dos apontados aqui, será observado no local. 


\begin{tabular}{|c|c|c|c|}
\hline Piezocerâmica & PZT5 & \multicolumn{2}{|c|}{ Alumino } \\
\hline$c_{11}^{E}\left(10^{10} \mathrm{~N} / \mathrm{mm}^{2}\right)$ & 12,10 & $E_{0}\left(10^{9} \mathrm{~N} / \mathrm{m}^{2}\right)$ & 7,00 \\
\hline$c_{12}^{E}\left(10^{10} \mathrm{~N} / \mathrm{mm}^{2}\right)$ & 7,54 & $v$ & 0,33 \\
\hline$c_{13}^{E}\left(10^{10} \mathrm{~N} / \mathrm{mm}^{2}\right)$ & 7,52 & \multicolumn{2}{|c|}{ Cobre } \\
\hline$c_{33}^{E}\left(10^{10} \mathrm{~N} / \mathrm{mm}^{2}\right)$ & 11,10 & $E_{0}\left(10^{9} \mathrm{~N} / \mathrm{m}^{2}\right)$ & 11,50 \\
\hline$c_{44}^{E}\left(10^{10} \mathrm{~N} / \mathrm{mm}^{2}\right)$ & 2,11 & $v$ & 0,31 \\
\hline$c_{66}^{E}\left(10^{10} \mathrm{~N} / \mathrm{mm}^{2}\right)$ & 2,26 & & \\
\hline $\mathrm{e}_{13}\left(C / m^{2}\right)$ & $-5,40$ & & \\
\hline $\mathrm{e}_{33}\left(C / m^{2}\right)$ & 15,80 & & \\
\hline $\mathrm{e}_{15}\left(C / m^{2}\right)$ & 12,30 & & \\
\hline$\varepsilon_{11}^{S} / \varepsilon_{0}$ & 916 & & \\
\hline$\varepsilon_{33}^{S} / \varepsilon_{0}$ & 830 & & \\
\hline
\end{tabular}

Tabela 6.1- Propriedade dos materiais.

\subsection{Influência dos parâmetros do MOT no projeto do Atuador}

Neste item verificamos através do APFLEX o que acontece quando variamos os parâmetros do MOT aplicado aos projetos de atuadores piezelétricos flextensionais, como: valor inicial de densidades, a restrição de volume, o coeficiente $w$ e a discretização do domínio inicial.

Portanto, todos os exemplos ilustram um projeto diferente de um atuador piezelétrico flextensional. Assim, o primeiro exemplo permite verificar se as topologias obtidas variam sensivelmente com diferentes valores de densidades iniciais. O segundo exemplo considera o projeto de um atuador flextensional e ilustra as topologias ótimas obtidas para quatro casos diferentes de restrição do volume de material $\left(V^{*}\right)$ do atuador. O terceiro exemplo descreve um projeto de um atuador flextensional, no qual são obtidas topologias ótimas para três diferentes valores do coeficiente $w$ da Eq.(4.30). No último exemplo são observados os resultados obtidos 
em três diferentes malhas de elementos finitos, geradas para o mesmo domínio inicial e mesmas condições iniciais de carregamento, restrições e geometria. Considerando que o material da estrutura acoplada para todos os exemplos descritos acima é o alumínio (Tab. 6.1).

\subsubsection{Exemplo 1 - sensibilidade ao valor inicial de densidades}

A Fig. 6.1 mostra o domínio inicial de projeto de um atuador piezelétrico flextensional (SILVA; NISHIWAKI; KIKUCHI, 1999), onde devido à simetria do atuador (em relação à direção horizontal e vertical) somente um quarto do domínio é apresentado. Os pontos de aplicação da carga elétrica, do deslocamento desejado e sua direção (ponto $B$ ) e das restrições de deslocamentos também são mostrados na mesma figura. Esse domínio é discretizado numa malha com 1200 elementos finitos isoparamétricos quadrados de 4 nós.

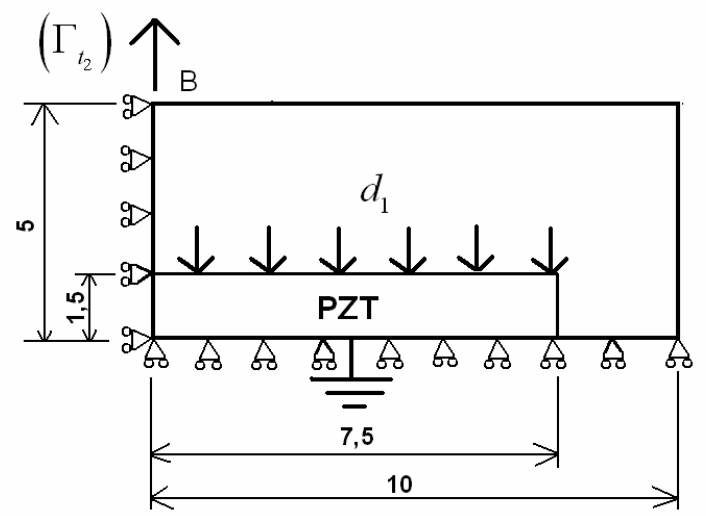

Figura 6.1 - Domínio inicial de projeto do atuador.

O coeficiente $w$ da Eq. (4.24) (função objetivo do problema de otimização) é igual à 0,8 , e a restrição de volume é igual à $20 \%$ do volume inicial (considerando o domínio totalmente sólido) e os valores iniciais para as densidades são: 0,10; 0,20; 
0,30 e aleatório para todos os elementos.

A seguir, são ilustradas nas Fig. 6.2 as configurações de topologias ótimas obtidas, através do APFLEX, para os quatro diferentes casos de densidade iniciais.

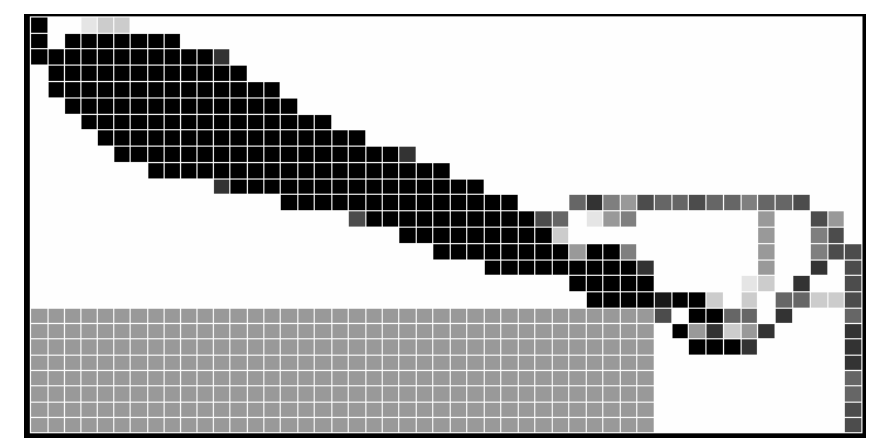

a) valor de densidade inicial igual à 0,10 .

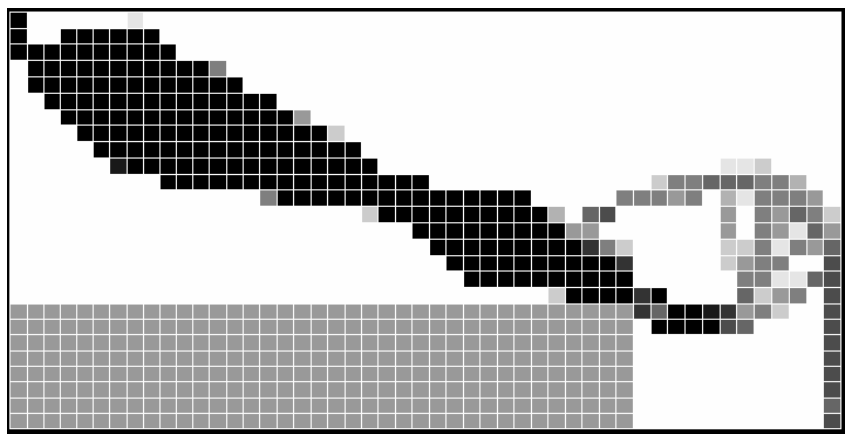

b) valor de densidade inicial igual à 0,20 .

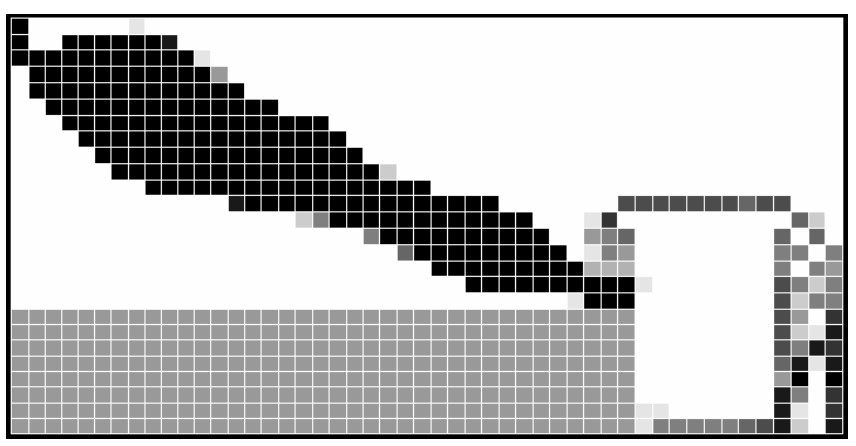

c) valor de densidade inicial igual à 0,30 . 


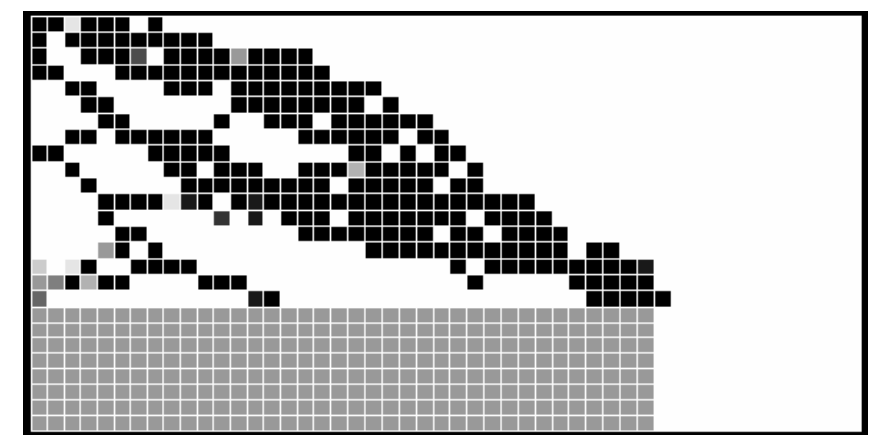

d) valor aleatório para as densidades iniciais de cada elemento do domínio inicial (valor entre 0,001 e 1,0 ).

Figura 6.2- Topologia Ótima para diferentes valores de densidades iniciais.

O MOT busca o máximo da função objetivo até convergir, no entanto, esta função possui vários pontos de máximo locais e globais (BENDSØE, 1989), conseqüentemente, o MOT pode convergir para diferentes pontos de máximos dependendo do ponto inicial que é fornecido ao software. Por exemplo, na Fig. 6.2 (d), cada variável de projeto recebeu um valor inicial aleatório, mostrando que para esta situação o método é sensível a este valor inicial de densidades, no entanto, analisando a Fig. 6.2 (a) até (c), a topologia da estrutura flexível é menos sensível do que o resultado obtido na Fig. 6.2 (d), devido ao fato de todas as variáveis de projeto receberem o mesmo valor inicial de densidades.

Pode-se verificar através dos gráficos de convergência, mostrado na Fig. 6.3, que a transdução média é maximizada e a flexibilidade média é minimizada. 

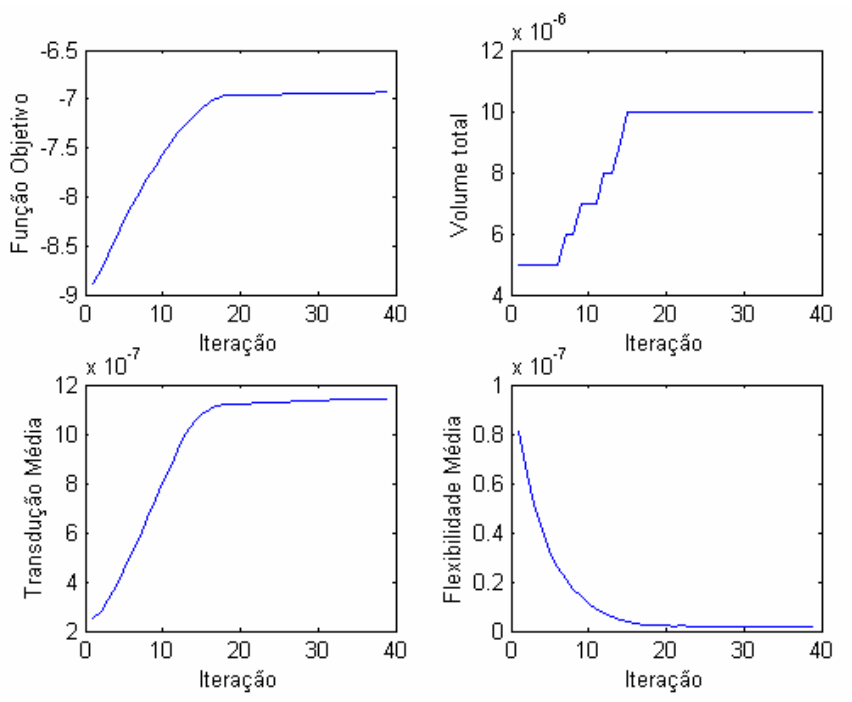

a) valor inicial de densidades $=0.10$.
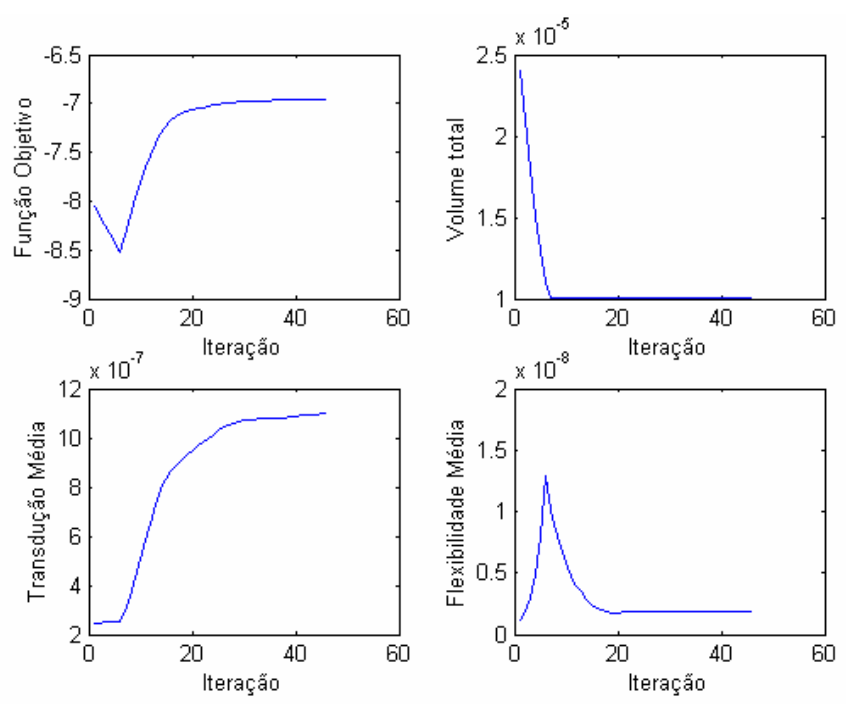

b) valor inicial de densidades aleatório.

Figura 6.3 - Gráficos de convergência.

\subsubsection{Exemplo 2 - variação da restrição de volume}

A Fig. 6.4 mostra o domínio inicial de projeto de um atuador piezelétrico 
flextensional (SILVA; NISHIWAKI; KIKUCHI, 1999), onde devido à simetria do atuador (em relação à direção horizontal e vertical) somente um quarto do domínio é apresentado. Os pontos de aplicação da carga elétrica, do deslocamento desejado e sua direção e das restrições de deslocamentos também são mostrados na mesma figura. Esse domínio é discretizado numa malha com 1200 elementos finitos isoparamétricos quadrados de 4 nós.

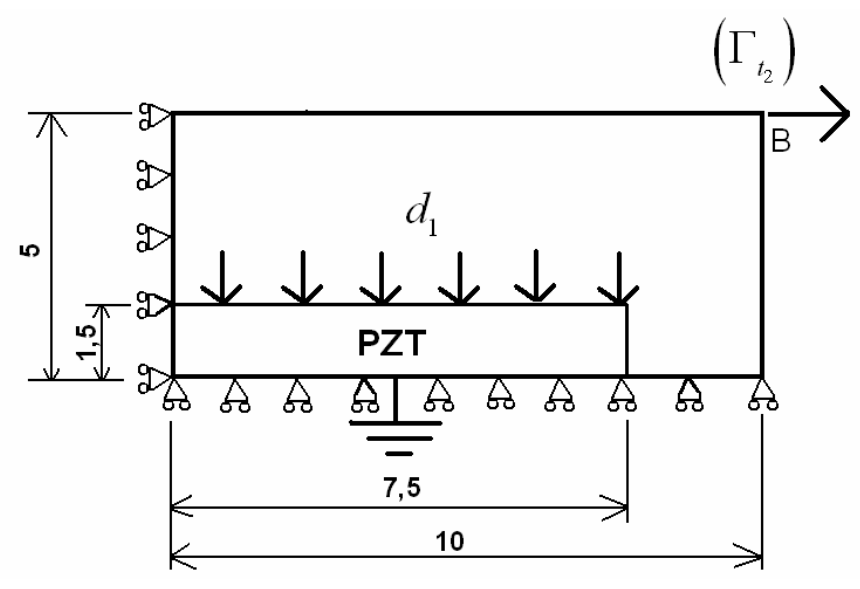

Figura 6.4 - Domínio inicial de projeto do atuador.

O coeficiente $w$ da Eq. (4.30) (função objetivo do problema de otimização) é igual à 0,8 . O volume de material é restringido em $10 \%, 15 \%, 20 \%$ e $30 \%$ do volume inicial (considerando o domínio totalmente sólido) e o valor inicial para as densidades é 0,15 .

A seguir, são ilustradas na Fig. 6.5 as configurações de topologias ótimas obtidas, através do APFLEX, para os quatro diferentes casos de restrição no volume do atuador. 

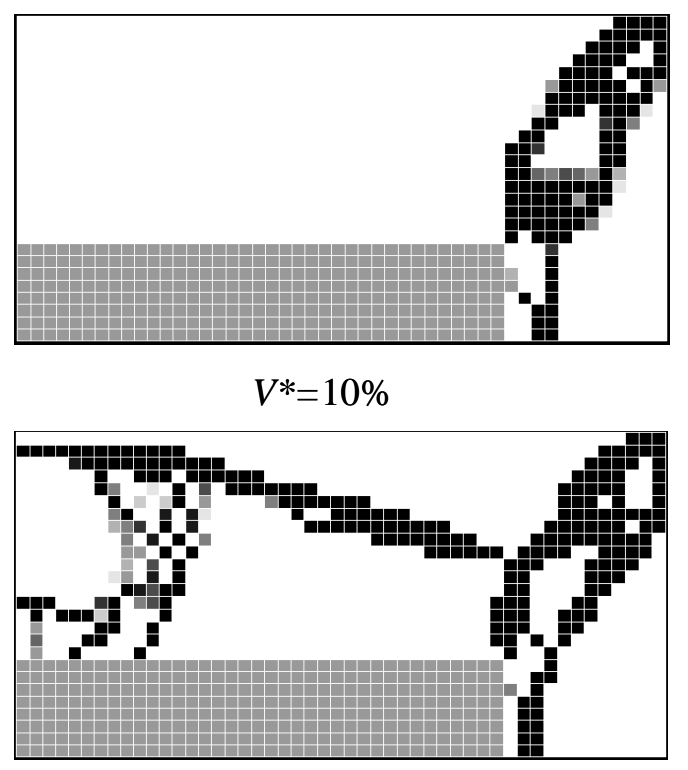

$V^{*}=20 \%$
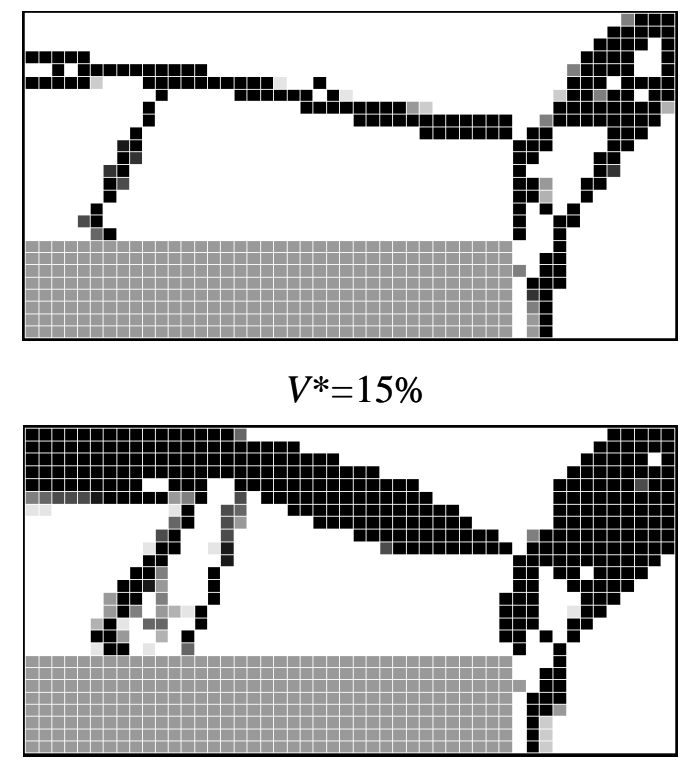

$V^{*}=30 \%$

Figura 6.5 - Efeito da variação da restrição de volume no projeto do Atuador Piezelétrico Flextensional.

Pode-se concluir, observando a Fig. 6.5, que a topologia da estrutura flexível principal (ver, Fig. 6.5 para $V^{*}=10 \%$ ) se mantém em todos os casos de restrição de volume de material, ou seja, a distribuição de material no interior do domínio inicial converge sempre para a mesma região. Contudo fica claro que a configuração ótima em cada caso é modificada, pois o MOT distribui material no interior do domínio fixo para atender as condições de projeto em cada caso. Percebese, que quando o MOT tem mais material para distribuir no interior do domínio (valores de $V^{*}$ do menor para o maior), o método acaba concentrando material próximo ao ponto $B$ para garantir rigidez suficiente para que o atuador suporte os deslocamentos gerados pela piezocerâmica (ver Tab. 6.2), enquanto atua em um determinado objeto na região do ponto $B$. Percebe-se que a região da simetria vertical (lado esquerdo do domínio inicial), inicialmente exige pouca quantidade de material, pois essa região proverá a flexibilidade necessária para o movimento do atuador.

No entanto, pode se notar que estes resultados possuem um ponto de articulação principal, que liga a estrutural principal com a secundária e que é comum 
a todos os exemplos (ver Fig. 6.6). Portanto, pode-se concluir que o MOT busca distribuir material no interior do domínio fixo de forma a manter a mesma flexibilidade (ver Tab. 6.2).

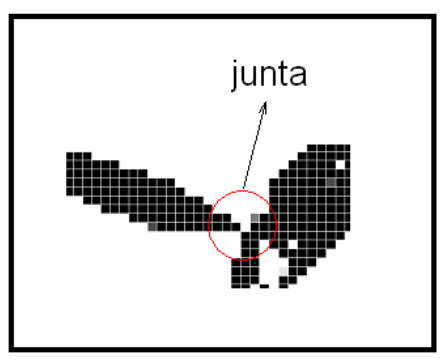

Figura 6.6 - Ponto principal de movimento da estrutura flexível da Fig. 6.5.

Recentemente, Poulsen (2002) introduziu um filtro que elimina os pontos de articulação (ou juntas).

\begin{tabular}{|c|c|c|c|c|}
\hline & $V^{*}=10 \%$ & $V^{*}=15 \%$ & $V^{*}=20 \%$ & $V^{*}=30 \%$ \\
\hline $\begin{array}{c}\text { Transdução } \\
\text { média }\left(10^{-7} \mathbf{J}\right)\end{array}$ & 5,94 & 6,00 & 6,30 & 6,49 \\
\hline $\begin{array}{c}\text { Flexibilidade } \\
\text { média }\left(10^{-9} \mathbf{J}\right)\end{array}$ & 7,18 & 3,70 & 3,05 & 2,80 \\
\hline
\end{tabular}

Tabela 6.2- Valores da Transdução média e Flexibilidade média para diferentes valores de $V^{*}$ para os atuadores deste exemplo.

Pode-se verificar através dos gráficos de convergência, mostrado na Fig. 6.7, que a transdução média entre o campo de aplicação da carga e o ponto $B$ é maximizada, o que significa maximizar o deslocamento neste ponto. Já a energia de 
deformação (relacionada com a flexibilidade média) no ponto $B$ é minimizada, significando que a rigidez foi maximizada, nas condições iniciais impostas no projeto.
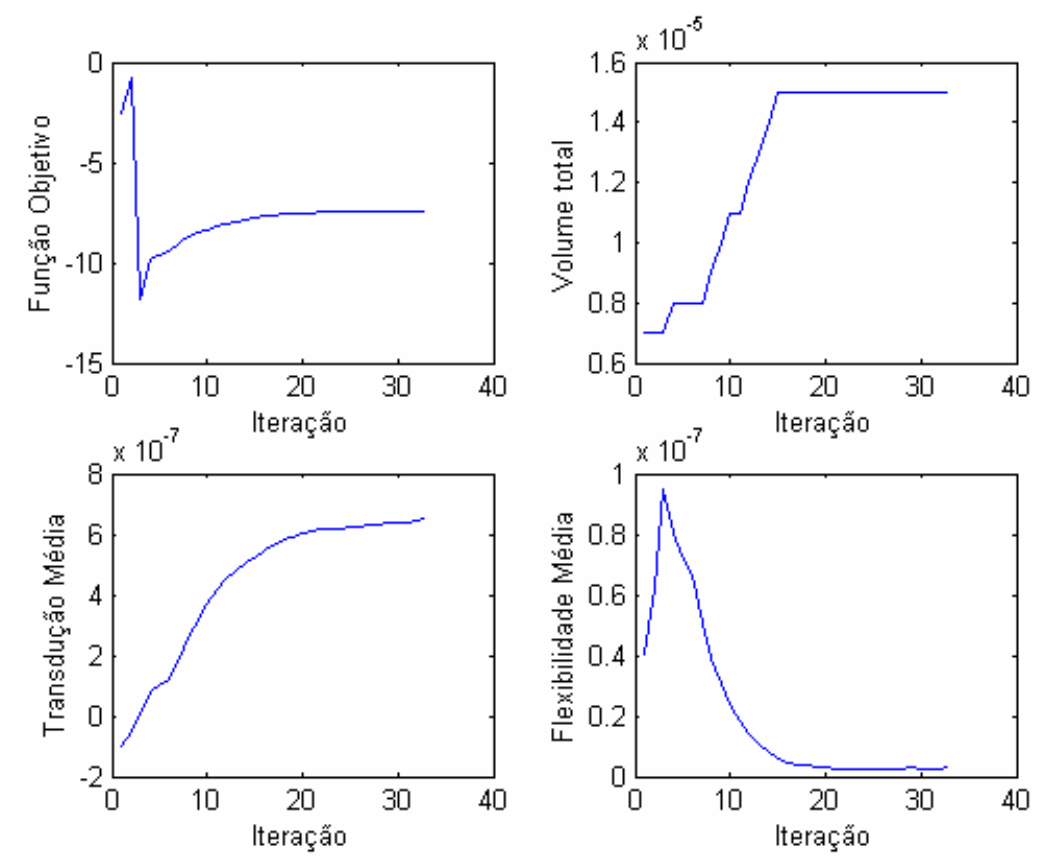

Figura 6.7 - Gráficos de convergência $\left(V^{*}=20 \%\right)$.

\subsubsection{Exemplo 3 - variação do coeficiente $w$}

A Fig. 6.8 deste exemplo mostra o domínio inicial de projeto do atuador (SILVA; NISHIWAKI; KIKUCHI, 1999). Somente um quarto do domínio inicial é desenhado, devido à simetria do atuador (em relação à direção horizontal e vertical). A carga elétrica, o deslocamento desejado e as restrições de deslocamentos são mostrados na mesma figura. O domínio é discretizado numa malha com 1200 elementos finitos isoparamétricos quadrados de 4 nós, e os lados de simetria do domínio terão todos os nós restringidos nas direções especificadas para atender à condição de simetria. 


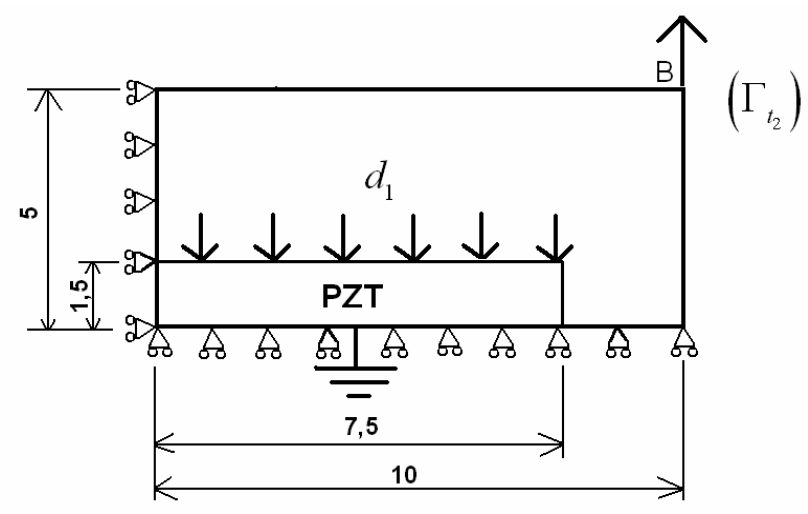

Figura 6.8 - Domínio inicial de projeto do atuador.

A intensidade da carga elétrica aplicada é $-1 \mu \mathrm{C} / \mathrm{m}^{2}$. O volume de material $V^{*}$ é restringido em $20 \%$ do volume inicial (considerando o domínio totalmente sólido) e o valor inicial para as densidades é 0,15 .

A Figura 6.9 ilustra as configurações de topologia ótima obtidas, através do APFLEX, para valores do coeficiente $w$ da função objetivo iguais a 0,25; 0,5 e 0,95.

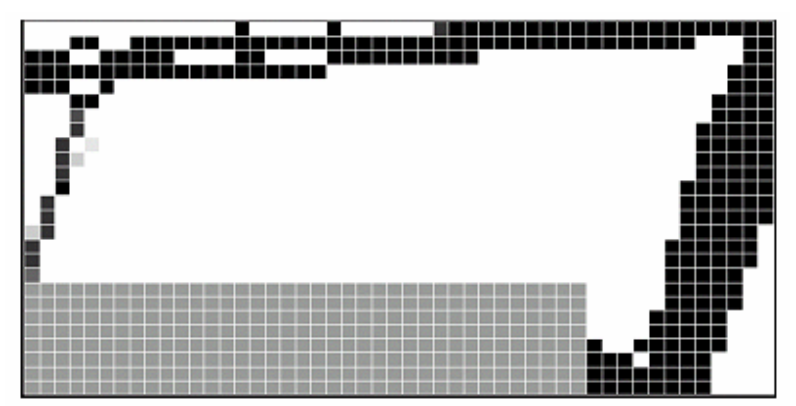

a) Topologia ótima para $w=0,25$. 


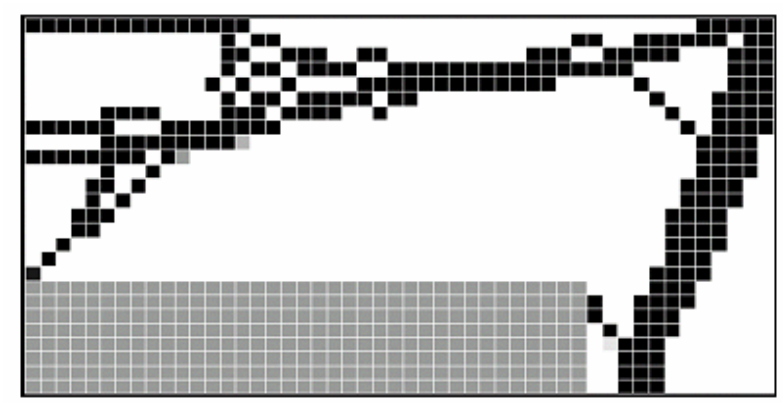

b) Topologia ótima para $w=0,5$.

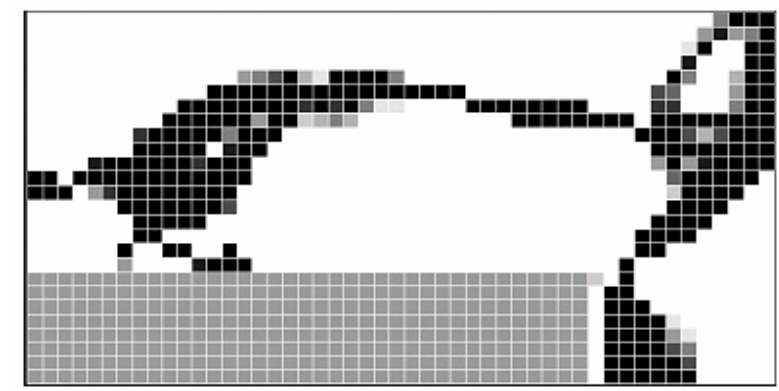

b) Topologia ótima para $w=0,95$.

Figura 6.9 - Efeito da alteração do coeficiente $w$ na OT do atuador.

Pode-se verificar através da Fig. 6.9, uma alteração na topologia do atuador conforme o valor de $w$ é modificado. Isso pode ser explicado devido ao fato de $w$ ser um coeficiente de atribuição de peso que permite ponderar a função objetivo, para que o atuador tenha mais flexibilidade e menos rigidez ou vice-versa. Quando diminuímos o valor de $w$ da Eq. (4.24) percebemos que há uma concentração maior de material na estrutura flexível na região de ligação do ponto $B$ a cerâmica piezelétrica (região direita do atuador), o que significa que a flexibilidade entre o ponto $B$ e à cerâmica (transdução média) diminui, enquanto a rigidez no ponto $B$ aumenta (ver Tab. 6.3). Conseqüentemente, conforme aumentamos o valor de $w$ da Eq. (4.24) notamos que a concentração de material na estrutura flexível na região de ligação do ponto $B$ à cerâmica piezelétrica (região direita do atuador) diminui, o que indica o aumento da flexibilidade do atuador e a diminuição da rigidez no ponto $B$ 
(ver Tab. 6.3). É claro que para valores de $w$ próximo de zero, o problema de otimização do atuador se torna semelhante aos problemas de otimização onde a função objetivo é maximizar a rigidez da estrutura (SUZUKI; KIKUCHI, 1991). Já para valores de $w$ próximos de 1 , tornam a configuração da topologia ótima quase indefinida, pois as descontinuidades (pontos de densidades quase nulas) que ocorrem na estrutura flexível, devido à alta flexibilidade, fazem com que o atuador seja inviável do ponto de vista prático.

\begin{tabular}{|l|l|l|}
\hline Valor de $\boldsymbol{w}$ & Transdução Média $\left(10^{-7} \mathbf{J}\right)$ & Flexibilidade Média $\left(10^{-10} \mathbf{J}\right)$ \\
\hline 0,25 & 1,24 & 1,86 \\
\hline 0,50 & 2,48 & 3,30 \\
\hline 0,95 & 9,60 & 113,76 \\
\hline
\end{tabular}

Tabela 6.3 - Valores da Transdução média e Flexibilidade média para diferentes valores de $w$ para os atuadores deste exemplo.

A Fig. 6.10 mostra os gráficos de convergência para a topologia ótima obtida com $w$ igual à 0,5 . Pode-se verificar através dos gráficos de convergência, que a transdução média entre os pontos de aplicação da carga elétrica e o ponto $B$ é maximizada, o que significa maximizar o deslocamento neste ponto. Já a flexibilidade média no ponto $B$ é minimizada, significando que a rigidez foi maximizada, nas condições iniciais impostas no projeto. 

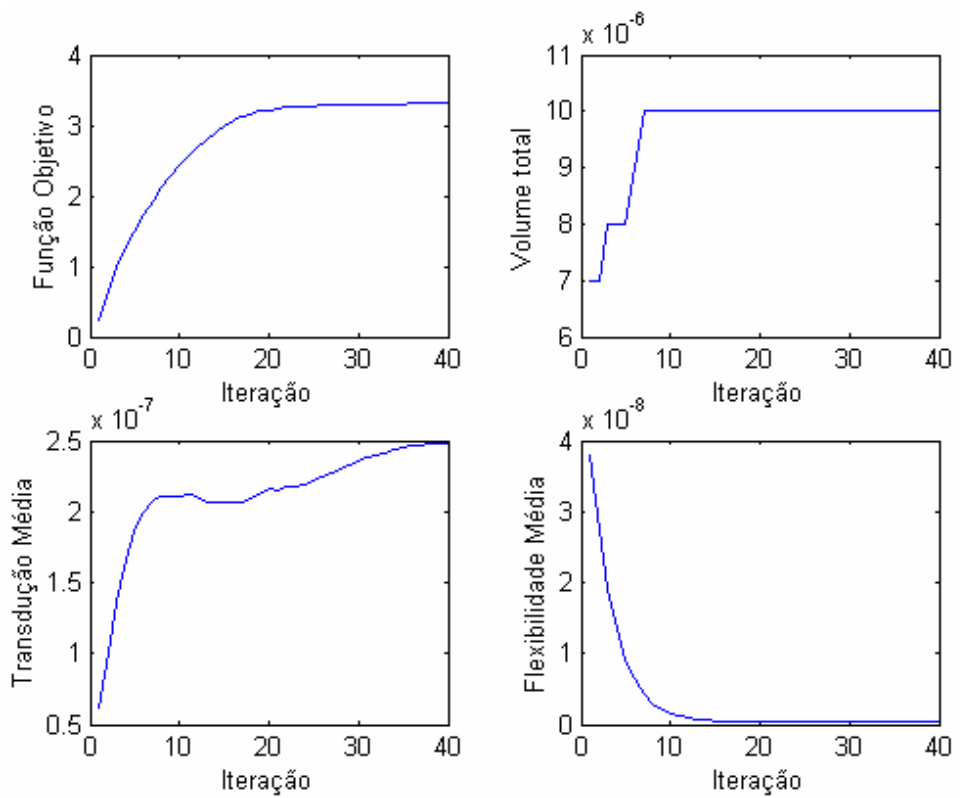

Figura 6.10 - Gráficos de convergência $(w=0,5)$.

\subsubsection{Exemplo 4 - variação na discretização do domínio inicial}

Neste exemplo verificamos a dependência do problema de otimização com relação ao tamanho da malha de elementos finitos utilizada. O domínio inicial de projeto do atuador, o ponto de aplicação do diferencial elétrico, o deslocamento desejado e as restrições de deslocamentos são mostrados na Fig. 6.11. Definimos este domínio inicial com o objetivo de obter através do APFLEX um atuador do tipo grampo (SILVA; NISHIWAK; KIKUCHI, 1999), onde ele atua de forma a prender, apertar ou agarrar um objeto localizado no ponto $B$. 


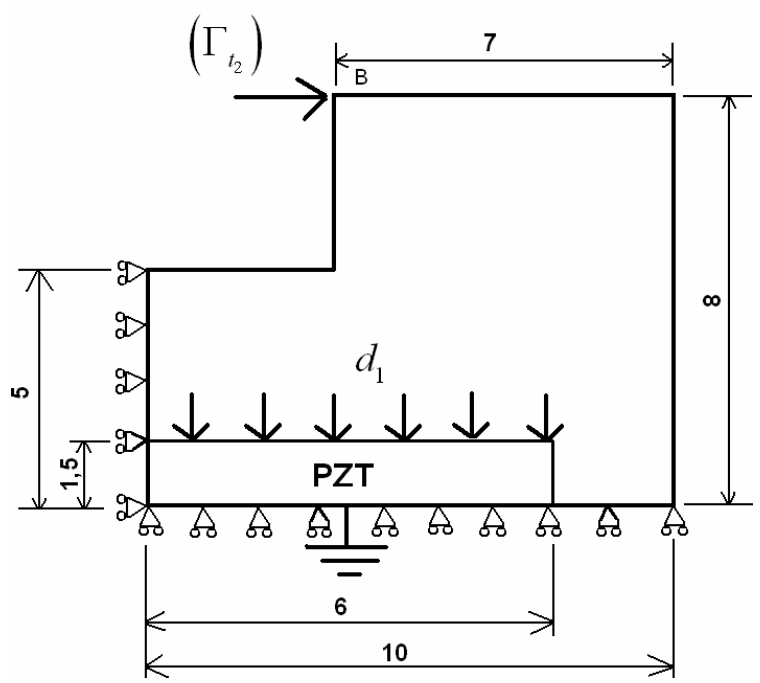

Figura 6.11 - Domínio inicial de projeto do atuador.

O volume de material é restringido em $20 \%$ do volume inicial (considerando o domínio totalmente sólido) e o valor inicial para as densidades é 0,15 . O coeficiente $w$ da função objetivo é igual à 0,7 . São utilizadas três malhas diferentes para a discretização do domínio inicial de projeto, sendo cada uma delas composta por 482, 818 e 1056 elementos finitos isoparamétricos quadrados de 4 nós, respectivamente. A Fig. 6.12 ilustra as configurações de topologia ótima obtidas.

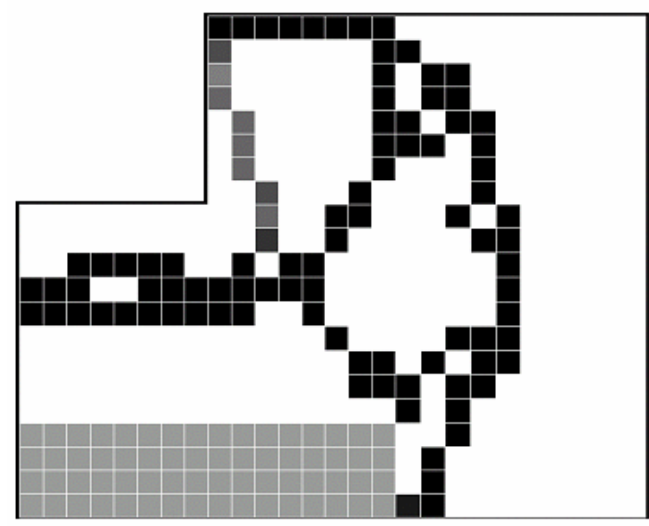

a) Topologia ótima para malha com 482 elementos 


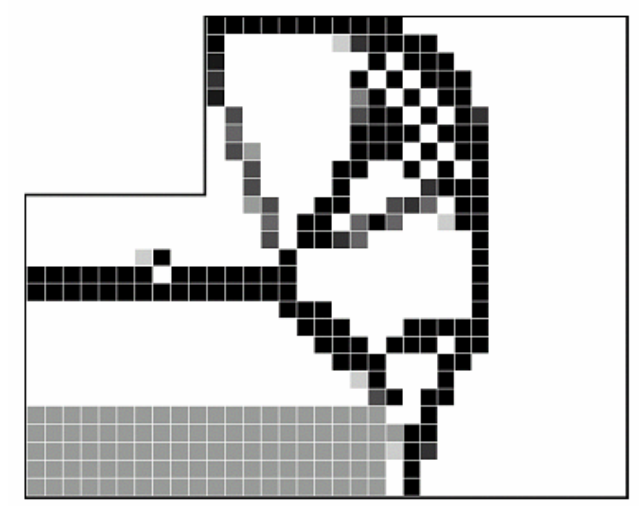

b) Topologia ótima para malha com 818 elementos

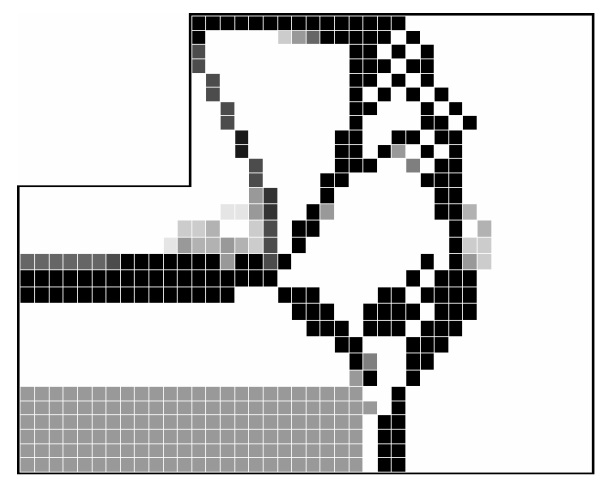

c) Topologia ótima para malha com 1056 elementos

Figura 6.12 - Configurações de topologia ótima para diferentes malhas.

Através das topologias mostradas na Fig. 6.12, percebe-se que a configuração de topologia ótima é semelhante nos três casos e que, obviamente, o contorno do atuador é mais definido com o aumento da discretização da malha (Fig. 6.12c). Neste exemplo, podemos observar que à medida que aumentamos a quantidade de elementos finitos, passa haver estruturas internas que são mais bem definidas na topologia, conseqüentemente, induzem a uma melhor descrição da geometria do atuador piezelétrico flextensional resultante. Porém, notamos também que à medida que aumentamos a discretização há a formação da "instabilidade xadrez" (Fig. 6.12b e 6.12c), aumentando a complexidade da topologia ótima. A 
"instabilidade xadrez" é um fenômeno que aparece devido a formulação do elemento finito utilizado (quadrilátero de 4 nós), que interpola o campo de deslocamentos utilizando um polinômio de baixa ordem (bilinear), considerada "pobre" pois não representa com precisão o comportamento ao cisalhamento do domínio discretizado (como já discutido extensivamente no capítulo 2). Portanto, através deste exemplo podemos concluir que o MOT aplicado ao projeto de atuadores é sensível ao aumento da discretização do domínio inicial (BENDSØE, 1995; NISHIWAKI; FRECKER; KIKUCHI, 1998). Uma solução para tornar o método independente da discretização do domínio inicial é a utilização de filtros espaciais, como já descrito no capítulo 4 e que será exemplificado no próximo item deste capítulo.

A Fig. 6.13 mostram os gráficos de convergência para uma das malhas (818 elementos). Observando os gráficos podemos concluir que a otimização ocorreu sob os mesmos aspectos observados nos exemplos anteriores, ou seja, a função objetivo foi maximizada, o volume convergiu para o valor da restrição, a transdução média foi maximizada, enquanto a flexibilidade média foi minimizada. Desta forma, para não se tornar repetitivo, nos próximos resultados não serão incluídos os gráficos de convergência.
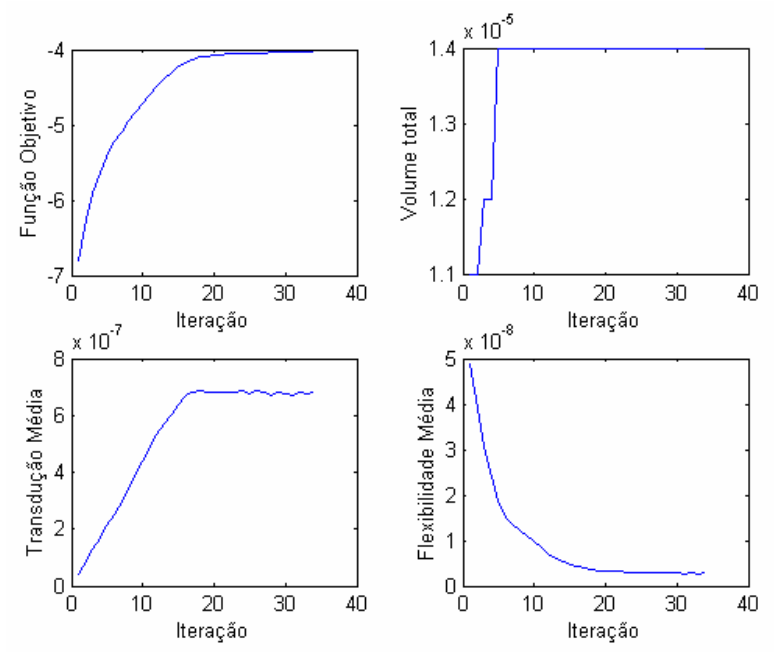

Figura 6.13 - Gráficos de convergência (malha com 818 elementos). 


\subsection{Formação e eliminação da "instabilidade xadrez"}

Modelos que apresentam maiores discretizações do domínio inicial permitem distinguir melhor o contorno da topologia ótima do atuador, além de descrever melhor o campo de deslocamentos e conseqüentemente o campo de deformações e tensões. Porém, também vimos que o aumento da discretização do domínio inicial induz a formação da "instabilidade xadrez". O que, acaba dificultando a interpretação das topologias ótima obtidas.

\subsubsection{Solução para tratar modelos com altas discretizações}

Mostraremos a seguir as novas configurações de topologias ótimas para os exemplos descritos no item 6.2 deste capítulo, considerando modelos com discretização superiores a 2000 elementos finitos. O primeiro resultado (Fig. 6.14), é a topologia ótima para o domínio da Fig. 6.2, onde consideramos o valor inicial de densidades igual à 0,15. A topologia ótima apresentada na Fig. 6.15 refere-se ao domínio inicial do exemplo do item 6.2.2 (Fig. 6.5), considerando a restrição de volume $\left(V^{*}\right)$ igual à $20 \%$. Já o terceiro resultado (Fig. 6.16) representa o domínio inicial ilustrado no exemplo do item 6.2.3 (Fig. 6.8), para o coeficiente $w$ igual à 0,8 e o volume $\left(V^{*}\right)$ igual à $15 \%$. O último resultado (Fig. 6.17) corresponde ao domínio inicial apresentado no exemplo do item 6.2.4 (Fig. 6.11), com valor de $w$ igual à 0,8 e uma discretização igual à 2556 elementos, e para os outros elementos uma discretização igual à 2278 elementos. Os demais parâmetros permaneceram os mesmos descritos no item 6.2. Observe que em alguns resultados (Fig. 6.16 e 6.17) há a formação da "instabilidade xadrez", cuja eliminação será exemplificada na seqüência. 


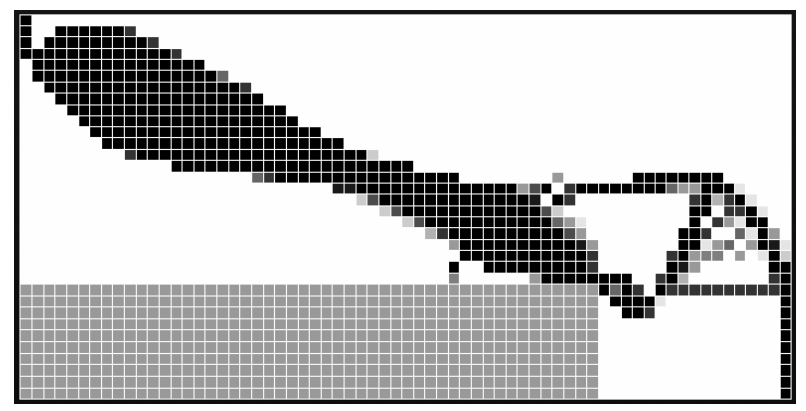

Figura 6.14 - Configuração de topologia ótima para o exemplo do item 6.2.1, considerando o valor do valor inicial de densidades igual à 0,15 e uma malha de 2500 elementos.

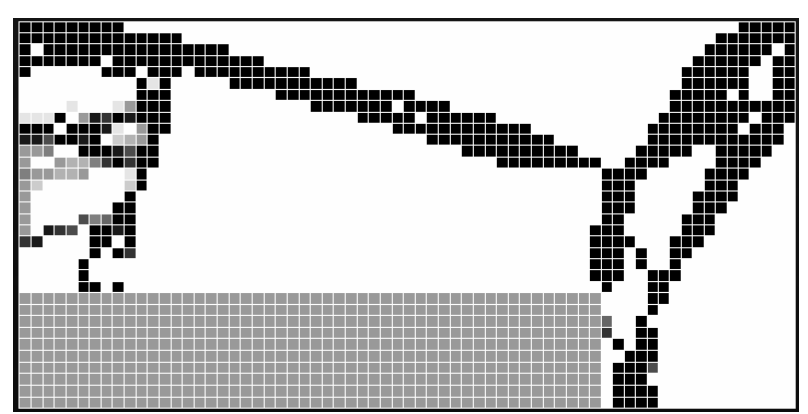

Figura 6.15 - Configuração de topologia ótima para o exemplo do item 6.2.2 considerando $V^{*}=20 \%$ e uma malha de 2500 elementos.

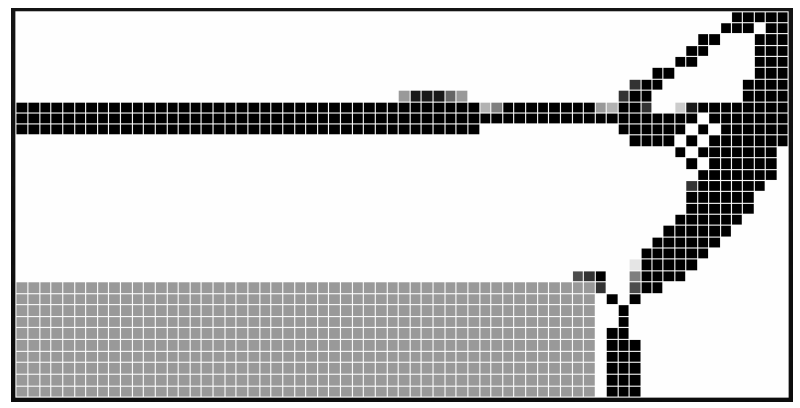

Figura 6.16 - Configuração de topologia ótima para o exemplo do item 6.2.3, considerando $w=0,80, V^{*}=15 \%$ e uma malha de 2500 elementos. 


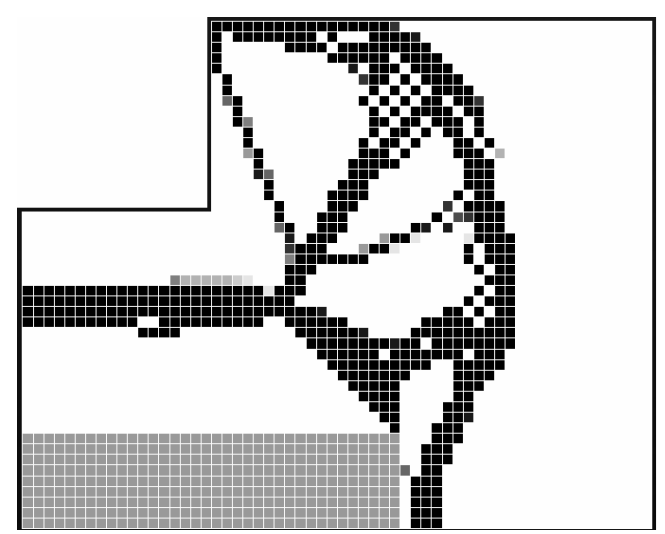

Figura 6.17 - Configuração de topologia ótima para o exemplo do item 6.2.4, considerando uma malha de 2556 elementos.

\subsubsection{Exemplos - eliminação da "instabilidade xadrez"}

Como detalhado no capítulo 4, a implementação na rotina do filtro espacial, evita a formação da "instabilidade xadrez", ou seja, o filtro suaviza a distribuição espacial das variáveis de projeto (a densidade de cada elemento da malha do MEF) ao longo do domínio inicial de projeto. $O$ filtro espacial minimiza a dependência do resultado final com a discretização da malha de elementos finitos e permite um controle da complexidade da topologia, ou seja, é possível a obtenção de topologias mais simples com a aplicação desse filtro.

O parâmetro de controle do filtro é o raio de abrangência e quanto maior for o raio, maior será a suavização da distribuição de densidades no interior do domínio inicial. Porém, devemos usar valores adequados para o raio, se este valor for muito pequeno corremos o risco de voltarmos ao problema da formação de "instabilidade xadrez" ou caso seja fornecido um raio muito grande, aumentamos a abrangência do filtro causando um aumento das áreas com densidades intermediárias. Neste trabalho, após várias aplicações, concluímos que sempre obtemos bons resultados usando um valor de raio que abrange os vizinhos comuns de arestas e cantos do elemento central (ver Fig. 5.2 do capítulo 5), o que corresponde a oito elementos vizinhos $j$ para um 
determinado elemento central $i$ na Eq. (5.20). Mais adiante ilustraremos esta conclusão.

Portanto, o filtro foi aplicado nos resultados apresentados no item anterior (Fig. 6.14 a 6.17) e as configurações de topologias ótimas mostradas nas Fig. 6.18 a 6.21 foram obtidas, com o raio abrangendo oito elementos.

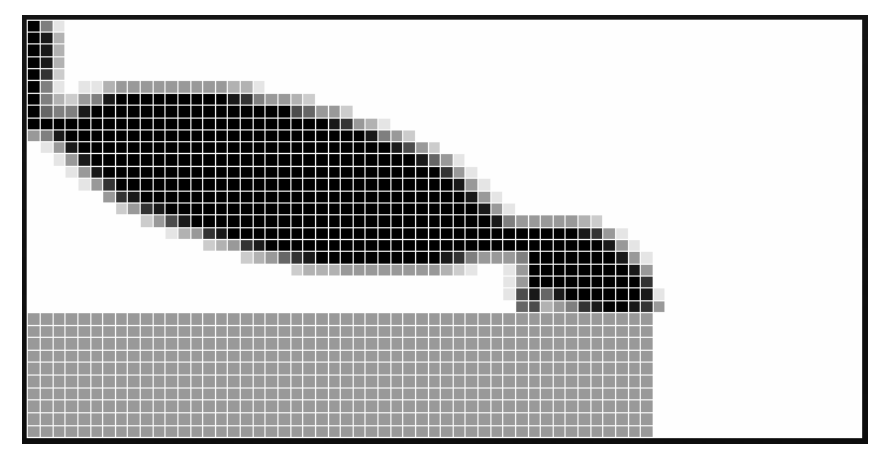

Figura 6.18 - Topologia ótima obtida com a utilização do filtro, para o domínio inicial do item 6.2.1.

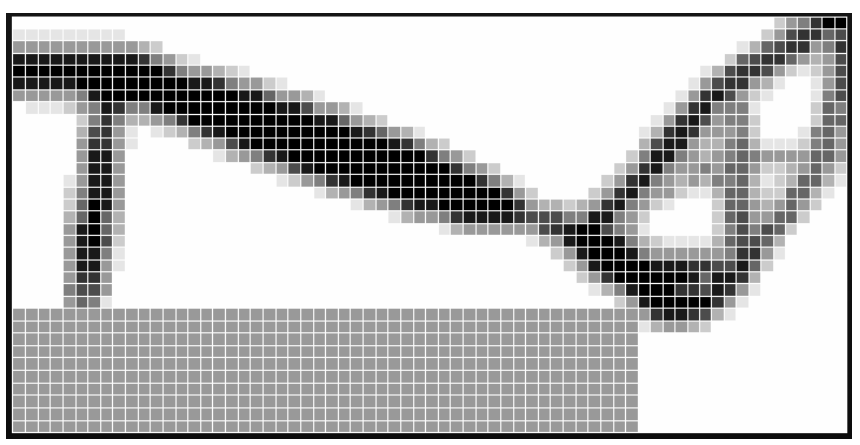

Figura 6.19 - Topologia ótima obtida com a utilização do filtro, para o domínio inicial do item 6.2.2. 


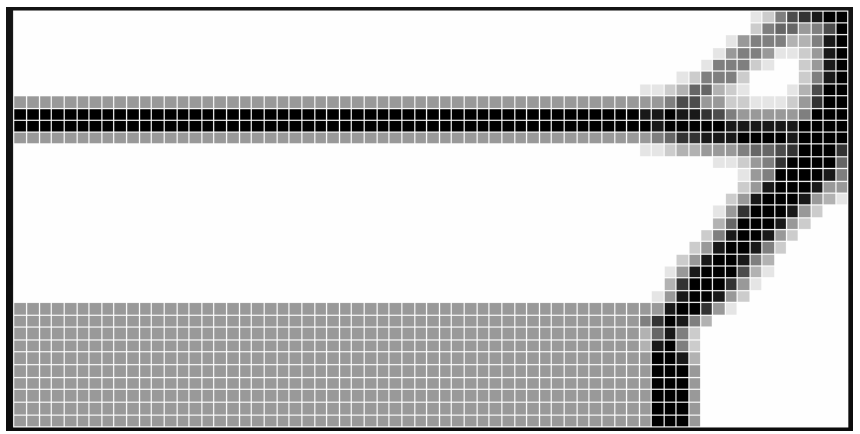

Figura 6.20 - Topologia ótima obtida com a utilização do filtro, para o domínio inicial do item 6.2.3.

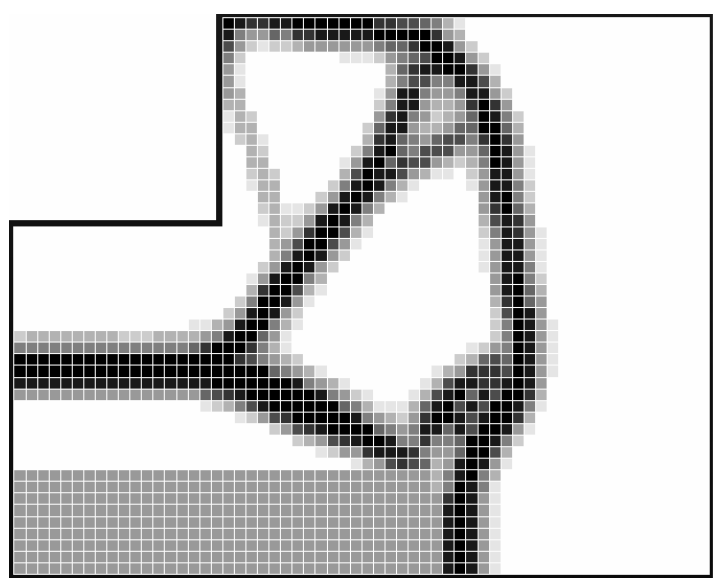

Figura 6.21 - Topologia ótima obtida com a utilização do filtro, para o domínio inicial do item 6.2.4.

\subsubsection{Redução das "escalas de cinza"}

Comparando os resultados apresentados nas Fig. 6.14 a 6.17 (sem aplicação do filtro) com os resultados mostrados nas Fig. 6.18 a 6.21 (com aplicação do filtro), podemos perceber através das configurações de topologias ótimas apresentadas que ao aplicarmos o filtro espacial eliminamos o fenômeno da "instabilidade xadrez", ou seja, a topologia ótima obtida é mais bem definida, isenta de vários reforços internos 
e pode ser interpretada facilmente.

Porém, observa-se um aumento da formação das "escalas de cinza" que, nos resultados apresentados nas Fig. 6.18 a 6.21, formam uma transição suave entre material sólido (região escura da topologia) e vazio (região clara da topologia), em torno do contorno da estrutura flexível. Isso ocorre, devido a uma distribuição mais uniforme de densidades ao longo do domínio. O filtro faz com que o método produza mais densidades com valores intermediários para definir a topologia ótima. Conceitualmente a solução ótima global é uma topologia que tende conter uma quantidade excessiva de "escalas de cinza". Contudo isto impossibilitaria a fabricação destes atuadores tanto em relação à geometria quanto ao custo de fabricação.

A aplicação do filtro permite ao método buscar a ótima solução global, mas há uma divergência de objetivos entre o fator $p$ (Eq. 2.6 do capítulo 2) e o filtro, pois o fator $p$ penaliza o excesso de densidades intermediarias ("escalas de cinza"), enquanto o filtro tende a aumentar as "escalas de cinza" reduzindo o fator $p$. Assim, o resultado é uma topologia ótima que apesar de ser mais bem definida, em relação aos resultados sem o filtro, apresenta uma quantidade considerável de "escalas de cinza" concentradas em torno do contorno. Uma solução bastante interessante para limpar essas "escalas de cinzas" é, após obter a topologia final, desligar o filtro e deixar o método prosseguir por mais algumas iterações (CARDOSO; FONSECA, 1999). Quando desligamos o filtro, a transição entre material sólido e materiais intermediários no interior do domínio inicial é mais brusca devido à penalidade imposta aos valores de densidade intermediárias $(p=3)$. Para obtermos resultados com o mínimo de escalas de cinza e sem a formação da instabilidade xadrez, a idéia é obter a quantidade de iterações necessárias para o método encontrar a solução ótima com o filtro aplicado em todas as iterações, após obtermos a iteração em que a função converge, então fazemos uma reanálise do problema, mas desligando o filtro com até 8 iterações do final, considerando como base a simulação anterior. Com isto, obtivemos bons resultados para todos os exemplos apresentados aqui.

A seguir, ilustramos a aplicação dessa metodologia para os resultados anteriormente mostrados nas Fig. 6.22 a 6.25. Logo após, são ilustrados outros 
exemplos que comprovam a eficácia desta metodologia.

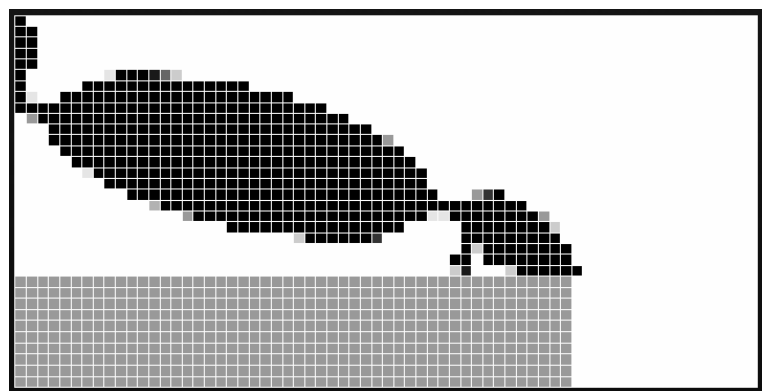

Figura 6.22 - Configuração final da topologia para o domínio inicial do item 6.2.1.

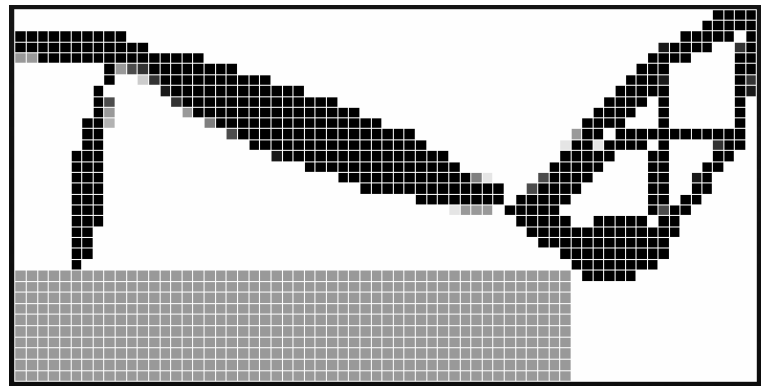

Figura 6.23 - Configuração final da topologia para o domínio inicial do item 6.2.2.

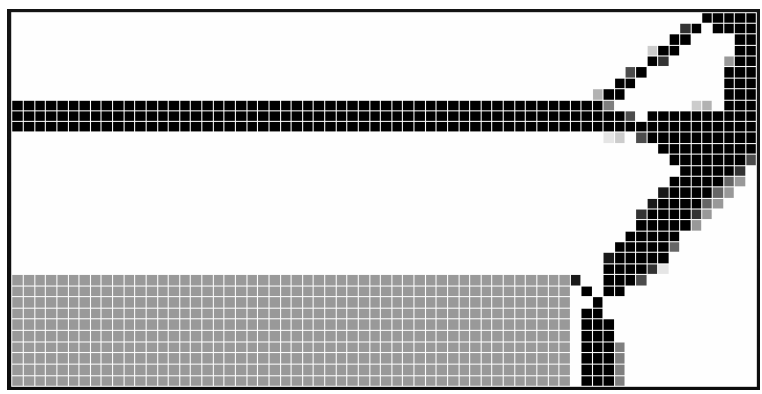

Figura 6.24 - Configuração final da topologia para o domínio inicial do item 6.2.3. 


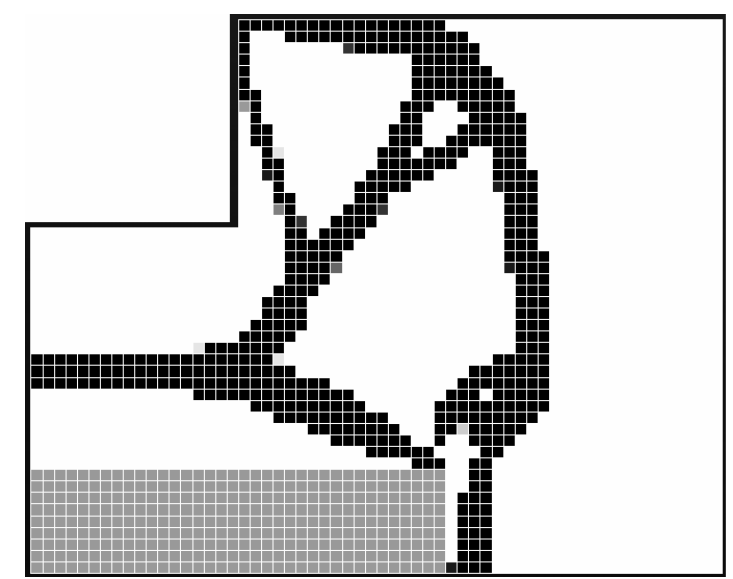

Figura 6.25 - Configuração final da topologia para o domínio inicial do item 6.2.4.

Como observado nas Fig. 6.22 a 6.25, agora as topologias ótimas são bem definidas, sem a presença da "instabilidade xadrez" e com uma quantidade ínfima de "escalas de cinza". Em algumas regiões do atuador, essas "escalas de cinza" formam uma configuração semelhante a "juntas", as quais são aceitáveis desde de que sejam interpretadas corretamente, para que o atuador tenha o desempenho esperado.

A Figura 6.26 mostra o domínio inicial de projeto e os pontos de aplicação de força e de restrições do atuador piezelétrico flexível do tipo "garra" (SILVA; NISHIWAKI; KIKUCHI, 1999) onde devido à simetria do atuador (em relação às direções horizontal e vertical) somente um quarto do domínio é apresentado. Os pontos de aplicação das cargas elétricas, do deslocamento desejado e das restrições de deslocamentos (nós fixos) também são mostrados na mesma figura. Esse domínio é discretizado numa malha com 1056 elementos finitos. 


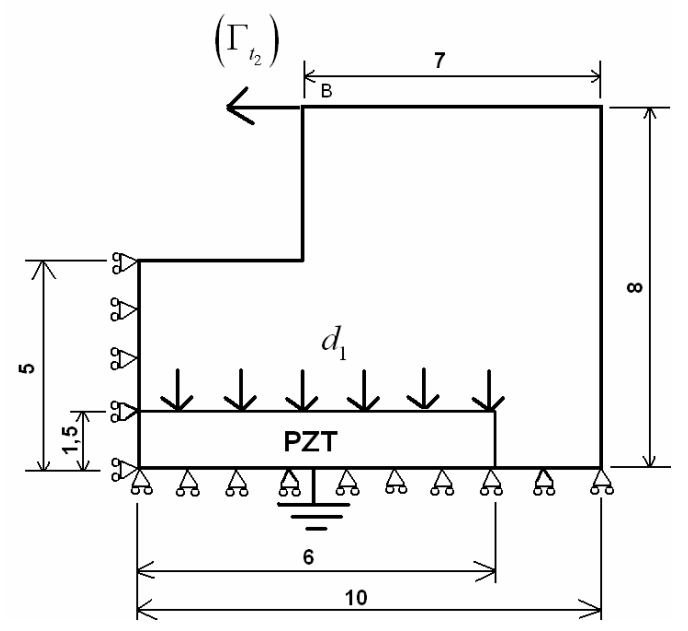

Figura 6.26 - Domínio inicial de projeto do atuador.

O valor da carga elétrica aplicada é $-1 \mu \mathrm{C} / \mathrm{m}^{2}$. O volume de material é restringido em $20 \%$ do volume inicial (considerando o domínio totalmente sólido) e o valor inicial para as densidades é 0,15 . O coeficiente $w$ da função objetivo é igual à 0,8. As Fig. 6.27 a 6.29 mostram, inicialmente, os resultados obtidos com e sem a utilização do filtro espacial e, em seguida, com o desligamento do filtro nas últimas iterações.

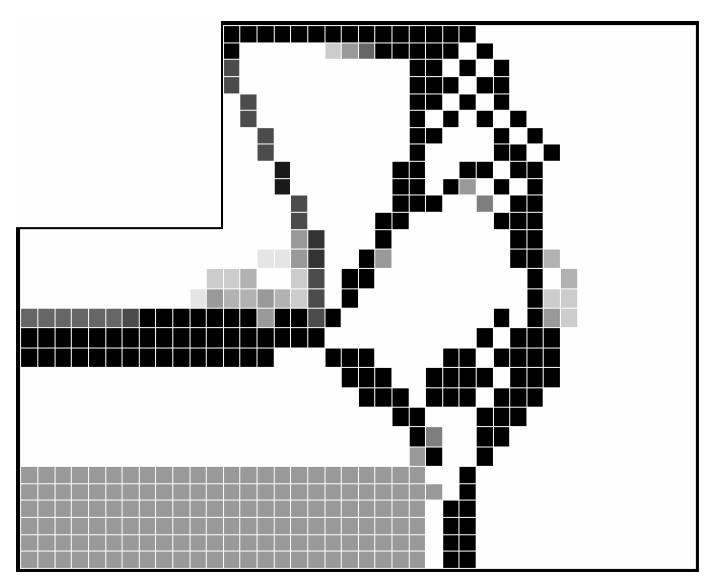

Figura 6.27 - Topologia ótima obtida SEM a utilização do filtro espacial. 


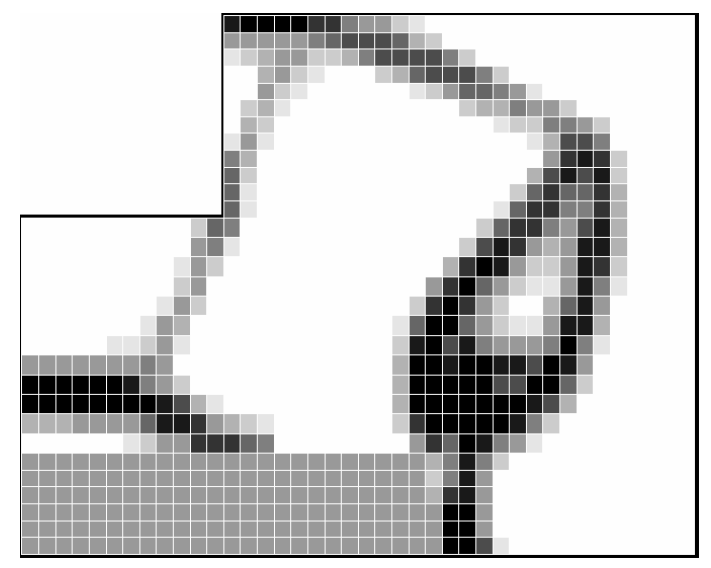

Figura 6.28 - Topologia ótima obtida COM a utilização do filtro espacial.

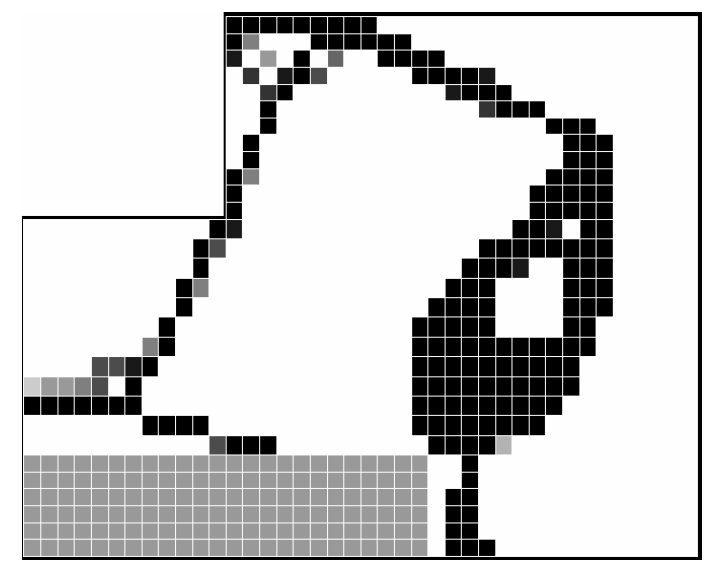

Figura 6.29 - Configuração final da topologia ótima obtida com o desligamento do filtro nas últimas iterações.

Conforme já vimos, o filtro permite a formação das "escalas de cinza" para definir uma distribuição suave de densidades ao longo do domínio. Este efeito é ampliado conforme aumentamos o raio de abrangência do filtro, pois mais elementos vizinhos $j$ são usados para definir a densidade do elemento central $i$ (veja Fig. 5.2 do capítulo 5). A Fig. 6.30 ilustra o efeito da variação do tamanho do raio de abrangência do filtro para o domínio inicial apresentado na Fig. 6.26. Nota-se que a 
topologia ótima é bem definida quando usado um raio que abrange oito elementos vizinhos em torno do elemento central, o que corresponde a usar somente os elementos que estão encostados nas arestas e nos cantos do elemento central, como já concluído no item 6.3.2.

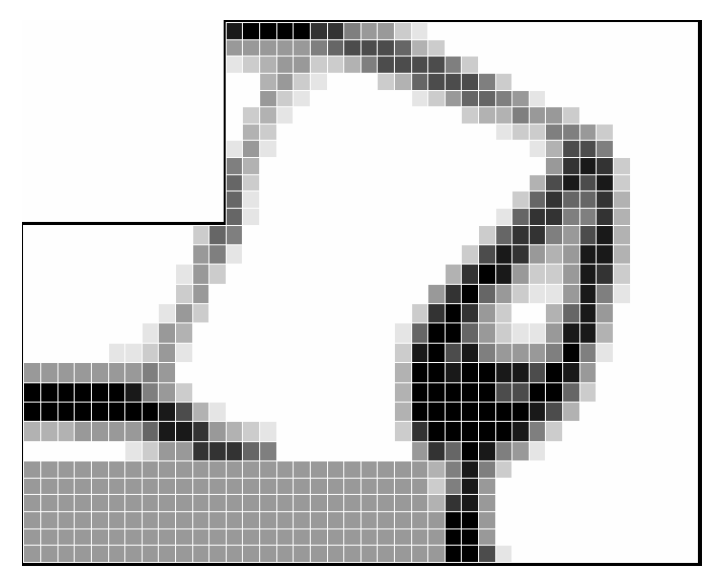

a) raio considerando 8 elementos vizinhos.

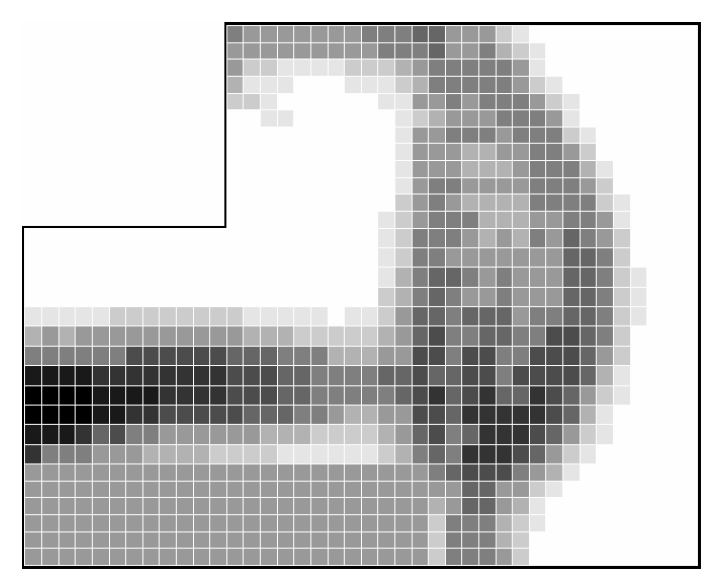

b) raio considerando 24 elementos vizinhos. 


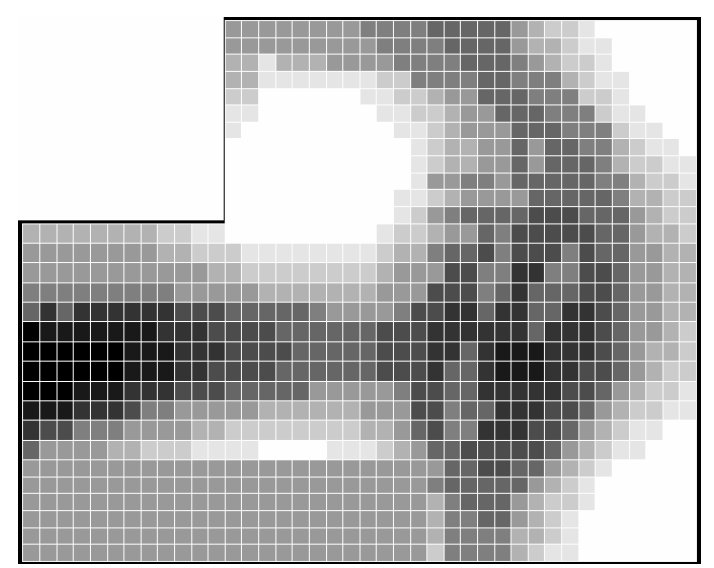

c) raio considerando 48 elementos vizinhos.

Figura 6.30 - Efeito da alteração do tamanho do raio de abrangência do filtro nos resultados.

\subsection{Verificação dos resultados obtidos}

Como mencionado anteriormente, o objetivo do item 6.2 e 6.3 foi mostrar como os parâmetros do problema de OT influenciam nos resultados e através destes exemplos, poder verificar se os resultados do MOT usando o modelo de material SIMP conferem com os resultados publicados em trabalhos nos quais foram utilizados o método da homogeneização como modelo de material. A comparação de resultados obtidos com modelos de materiais diferentes se deve ao fato de não haver outros trabalhos com as características do desenvolvido neste trabalho de mestrado.

O resultado mostrado abaixo (Fig. 6.31) é comparado com o obtido pelo APFLEX e ilustrado na Fig.6.14. 


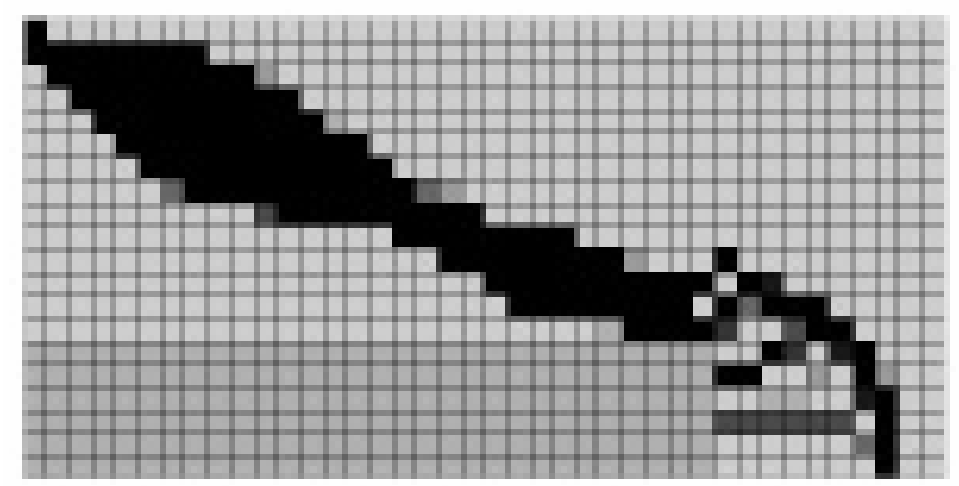

Figura 6.31 - Resultado da topologia ótima obtida por Silva; Nishiwaki; Kikuchi (2000), considerando condições semelhantes do item 6.2.1.

Comparando os resultados (Fig. 6.14 e Fig. 6.31), pode-se notar que a topologia ótima obtida para ambos os atuadores são idênticas, tanto para a estrutura principal quanto para a secundária. No entanto, se for comparar o resultado mostrado na Fig. 6.22 com os mostrados nas Fig. 6.14 e 6.31, verifica-se que o MOT usando o filtro espacial distribui material no interior do domínio de forma a eliminar as estruturas secundárias, e também altera a estrutura principal dando uma maior flexibilidade estrutural.

No segundo exemplo, é comparado a Fig. 6.32 com a Fig. 6.33, que representa a Fig. 6.16 sem as condições de simetria.

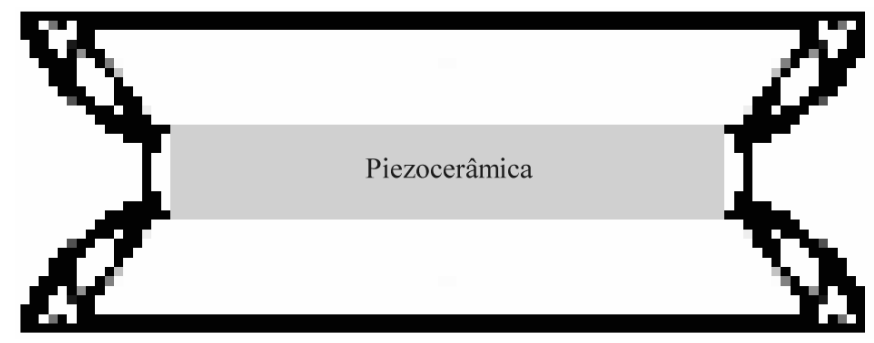

Figura 6.32 - Resultado da topologia ótima obtida Silva; Nishiwaki; Kikuchi (2000), considerando condições semelhantes do item 6.2.3. 


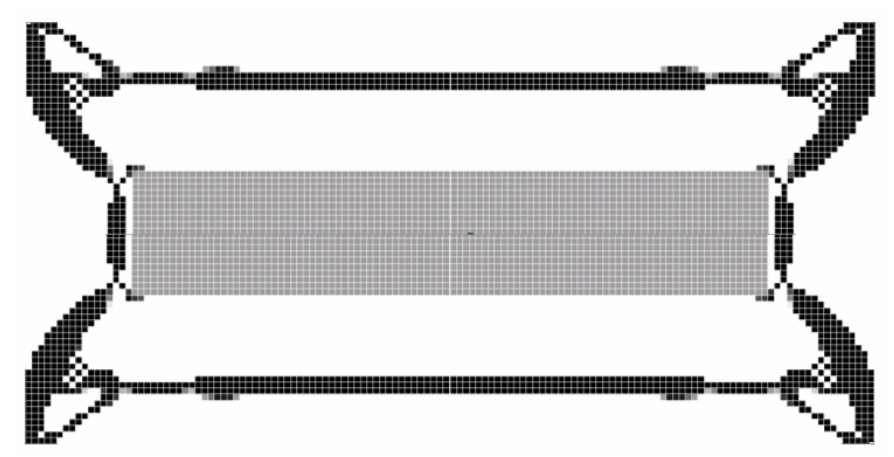

Figura 6.33 - Resultado da topologia ótima da Fig. 6.16 sem as condições de simetria.

Comparando os resultados (Fig. 6.32 e Fig. 6.33), percebe-se que a topologia ótima obtida para ambos os atuadores são próximas e também se comparado com o resultado mostrado na Fig. 6.24, verifica-se que a influência do filtro espacial neste exemplo é mínima.

Já no último exemplo, é comparado a Fig. 6.34 com a figura Fig.617.

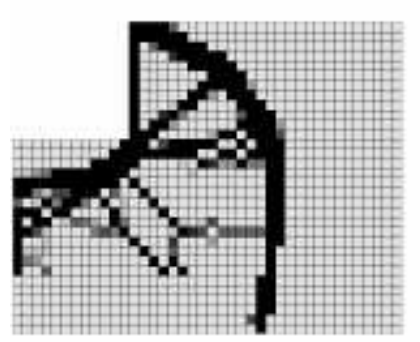

a)

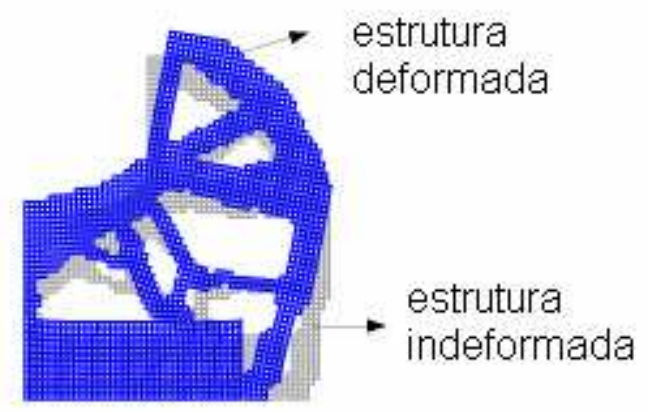

b)

Figura 6.34 - Resultado da topologia ótima obtida e deformada (SILVA;

NISHIWAKI; KIKUCHI, 1999), considerando condições semelhantes do item 6.2.4. 


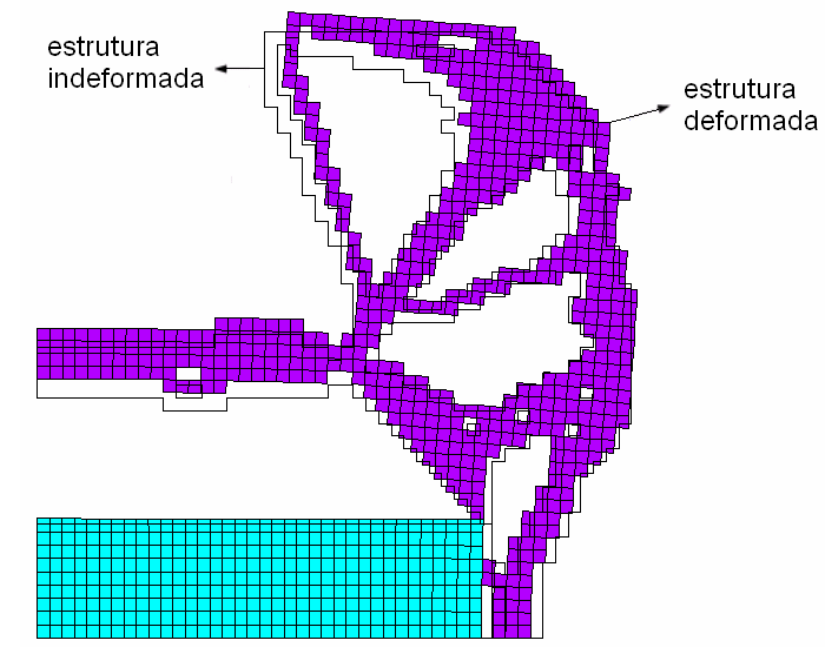

Figura 6.35 - Deformada da topologia ótima da Fig. 6.17.

Comparando os resultados (Fig. 6.17 e Fig. 6.34 (a)), pode-se notar que a topologia ótima obtida para ambos os atuadores são semelhantes, tanto para a estrutura principal quanto para a secundária. No entanto, se for comparar o resultado mostrado na Fig. 6.25 com os mostrados nas Fig. 6.17 e 6.34(a), verifica-se que o MOT usando o filtro espacial distribui material no interior do domínio de forma a eliminar as estruturas secundárias, e também obtém-se a estrutura principal melhor definida. As Fig. 6.34(b) e Fig. 6.35 mostram a deformada deste atuador.

\subsection{Projetos de Atuadores Piezelétricos Flextensionais}

Os resultados apresentados até aqui, como discutido no item anterior, conferem com os resultados descritos na literatura (SILVA; NISHIWAKI; KIKUCHI, 1999), o que atesta o APFLEX para aplicação do MOT para o projeto de atuadores piezelétricos flextensionais. Dessa forma, a próxima etapa tem como objetivo modelar e projetar atuadores piezelétricos flextensionais em aplicações dos mais variados tipos de atuadores, através do procedimento descrito no capítulo 2 (item 2.3 Fig. 2.2). No caso de se projetar estruturas microscópicas, pode ocorrer o 
problema das dimensões geométricas serem da ordem da dimensão do grão do material, o que faz com que as técnicas de modelagem e fabricação apresentadas aqui não sejam válidas, pois consideram um meio contínuo no interior do domínio. Nesse caso a formulação do MEF e a técnica de fabricação deve ser alterada de forma a levar em conta a dimensão dos grãos.

Neste item, será mostrado o detalhamento do modelo, como, área e direção de atuação, as condições de carregamento elétricos e mecânicos do domínio inicial de projeto do atuador até a fabricação dos atuadores usando o processo de corrosão química em chapas de cobre através do método de litografia (item 6.5.1). Durante o procedimento típico de projeto estrutural por OT, a interpretação é uma das tarefas que exige muita atenção, pois qualquer erro nessa operação pode comprometer o resultado da otimização. Geralmente, pode ser realizada por meio de processamento de imagem ou simplesmente desenhando o contorno sobre a imagem da topologia usando um CAD (desenho auxiliado por computador).

A interpretação dos resultados da OT não é uma atividade simples, principalmente se for realizada manualmente, pois existem alguns aspectos, como, por exemplo, a presença de "escalas de cinza", que mesmo ocorrendo numa quantidade muito pequena, dificultam a interpretação da topologia. O software implementado neste trabalho (APFLEX) gera para a configuração da topologia ótima, uma malha de elementos finitos em formato padrão do ANSYS, o que permite uma análise e verificação através do MEF do comportamento da topologia obtida, ou seja, se a topologia ótima obtida atende às condições exigidas no projeto. No entanto, o APFLEX não possui nenhum recurso que torne a interpretação um processo sistemático. Neste trabalho, a interpretação do contorno das topologias ótimas obtidas através do APFLEX, foi realizada manualmente, desenhando-se o contorno sobre a imagem da topologia usando um CAD. Na seqüência, é feita uma análise do atuador através do MEF, para verificar se a estrutura flexível esta convertendo, amplificando e direcionando os deslocamentos gerados pela piezocerâmica.

As cerâmicas piezelétricas utilizadas na análise experimental possuem dimensões de $7 \times 2 \times 1 \mathrm{~mm}^{3}$ e foram obtidas fatiando uma piezocerâmica de dimensões $30 \times 14 \times 1 \mathrm{~mm}^{3}$, utilizando uma máquina de corte a fio no Laboratório de Cerâmicas Ferroelétricas da UFSCar. O cobre é considerado como material da 
estrutura flexível (propriedades, ver Tab. 6.1), o volume de material é restringido em $20 \%$ do volume inicial (considerando o domínio totalmente sólido), o valor inicial para as densidades é 0,15 , o coeficiente $w$ da função objetivo é igual à 0,8 e a intensidade de carga elétrica aplicada nos eletrodos é de $1 \mu \mathrm{C} / \mathrm{m}^{2}$. Para as verificações realizadas no software ANSYS, foi aplicado uma tensão de 100 volts no eletrodo da piezocerâmica.

\subsubsection{Processo de fabricação}

Serão utilizadas técnicas de microfabricação disponíveis no laboratório de microfabricação do Laboratório Nacional de Luz Síncrotron (LNLS) em Campinas. Esse laboratório tem por objetivo propagar a técnica de microfabricação no Brasil. Trata-se de um laboratório aberto, onde os equipamentos da sala de microfabricação estão disponíveis com apoio técnico para serem usados por quem se propõem a trabalhar nessa área. Os gastos se limitam em geral à reagentes químicos diversos, substratos e despesas de viagem e acomodação para o LNLS e fotolitos. A técnica de microfabricação usada (MADOU, 1997) consiste em sensibilizar através de luz ultravioleta uma região na camada de polímero ("photoresist") que cobre o material no qual se deseja construir o micromecanismo (por exemplo, aço inox, cobre, alumínio). O polímero define a área que não deve ser corroída (ou em alguns casos, o oposto), ou seja, a topologia correspondente a geometria do micromecanismo que se deseja fabricar. Após a sensibilização, o micromecanismo é obtido por um processo de corrosão química ("etching"). Uma das etapas mais importante é a sensibilização da região na camada de polímero, pois a precisão de fabricação do "MEMS" depende da precisão com que se consegue iluminar a forma desejada do micromecanismo na camada de polímero. Para isso é necessário usar um fotolito. Esse fotolito define as regiões em que a luz ultravioleta pode passar e atingir o polímero. A fabricação desse fotolito é obtida através da contratação de serviços de terceiros. Uma opção de baixo custo na obtenção das máscaras é utilizar uma transparência impressa em impressora laser de boa qualidade (1200 dpi), ao invés do fotolito, no entanto a resolução final 
obtida na geometria do "MEMS" é menor. Portanto, fotolitos com alta resolução (permitem fabricar "MEMS" com alta resolução) tem custo mais elevado. A utilização de transparências com baixa resolução (permitem fabricar "MEMS" com resolução menor) tem custo menor. Dessa forma para o treinamento podem ser usadas as máscaras de baixa resolução e para o resultado final serão usados os fotolitos de alta resolução.

A sensibilização por ultravioleta e a corrosão química ("etching") são realizadas utilizando-se equipamentos do laboratório de microfabricação do LNLS para esse fim. As técnicas de microfabricação de "MEMS" são mais simples do que as técnicas usadas em microeletrônica, pois não exigem equipamentos sofisticados como sala limpa, etc.

Os atuadores foram fabricados em cobre (pelo fato de possuir uma boa flexibilidade) com dimensões de milímetros, com espessuras de 60; 100; $150 \mu \mathrm{m}$. Na medição dos deslocamentos dos atuadores piezelétricos flextensionais projetados serão utilizados alguns equipamentos do LNLS, como uma câmara CCD acoplada a um computador ("Probe Station" em inglês).

\subsubsection{Exemplos de atuadores piezelétricos flextensionais projetados}

\section{Exemplo 1}

A Fig. 6.36 mostra o domínio inicial de projeto do atuador piezelétrico flextensional, onde devido a simetria do atuador (em relação à direção horizontal e vertical) somente 1/4 do domínio é apresentado. Os pontos de aplicação das cargas elétricas, do deslocamento desejado e das restrições de deslocamentos também são mostrados na mesma figura. O domínio é discretizado numa malha com 2312 elementos finitos.

A Fig. 5.37 ilustra a configuração da topologia ótima obtida, através do APFLEX. Analisando-se a deformada da topologia ótima (Fig. 6.38), pode-se 
verificar que este tipo de atuador possui deslocamentos na área de atuação somente na direção determinada no projeto. Na Tab. 6.4 está os valores dos deslocamentos na direção $x$ e $y$ para o ponto de atuação e na Fig. 6.39 a distribuição das tensões de Von-Mises para o atuador. Na Fig. 6.39, é importante notar, que o MOT distribui material no interior do domínio inicial de projeto, de forma que hajam poucos pontos de concentração de tensões na estrutura. Por fim, o atuador pode ser fabricado e o protótipo é mostrado na Fig. 6.40.

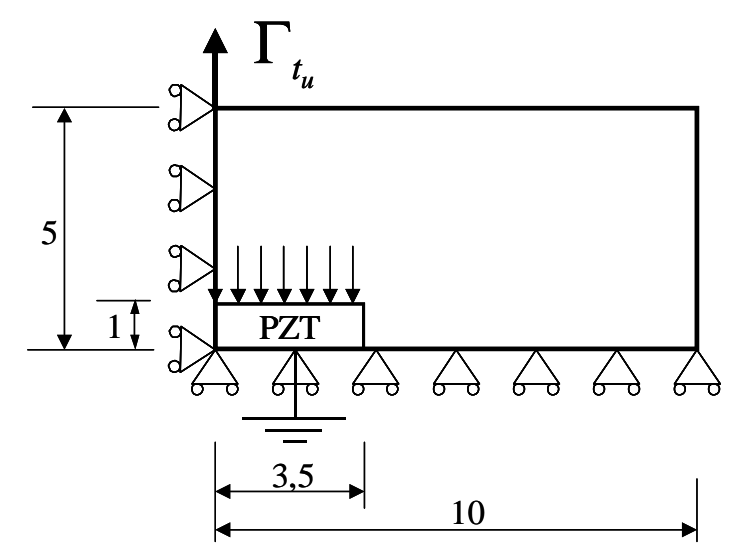

Figura 6.36 - Domínio inicial do atuador.

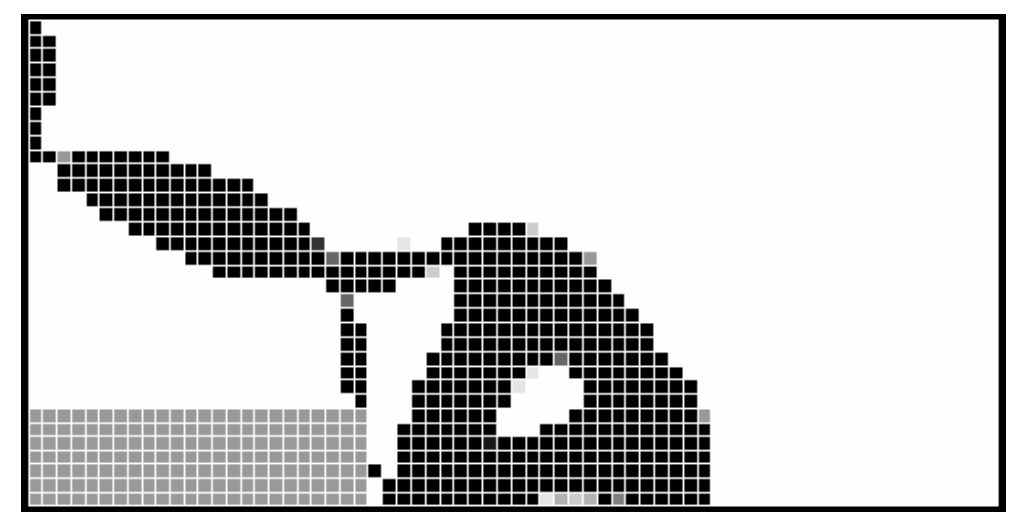

Figura 6.37 - Configuração da topologia ótima. 


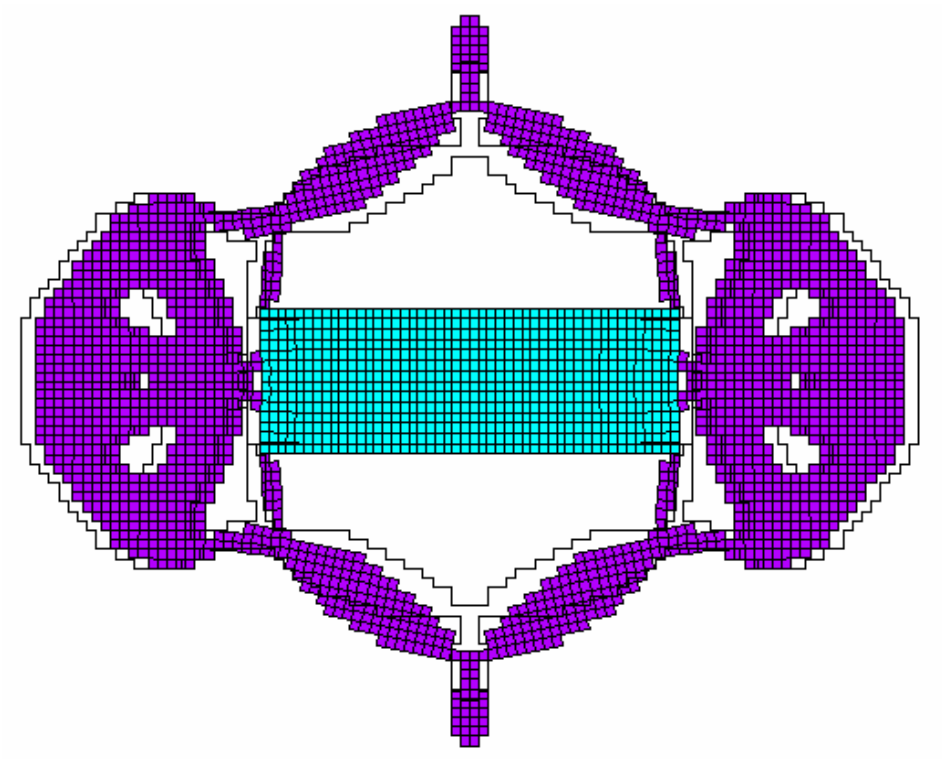

Figura 6.38 - Deformada da topologia ótima.

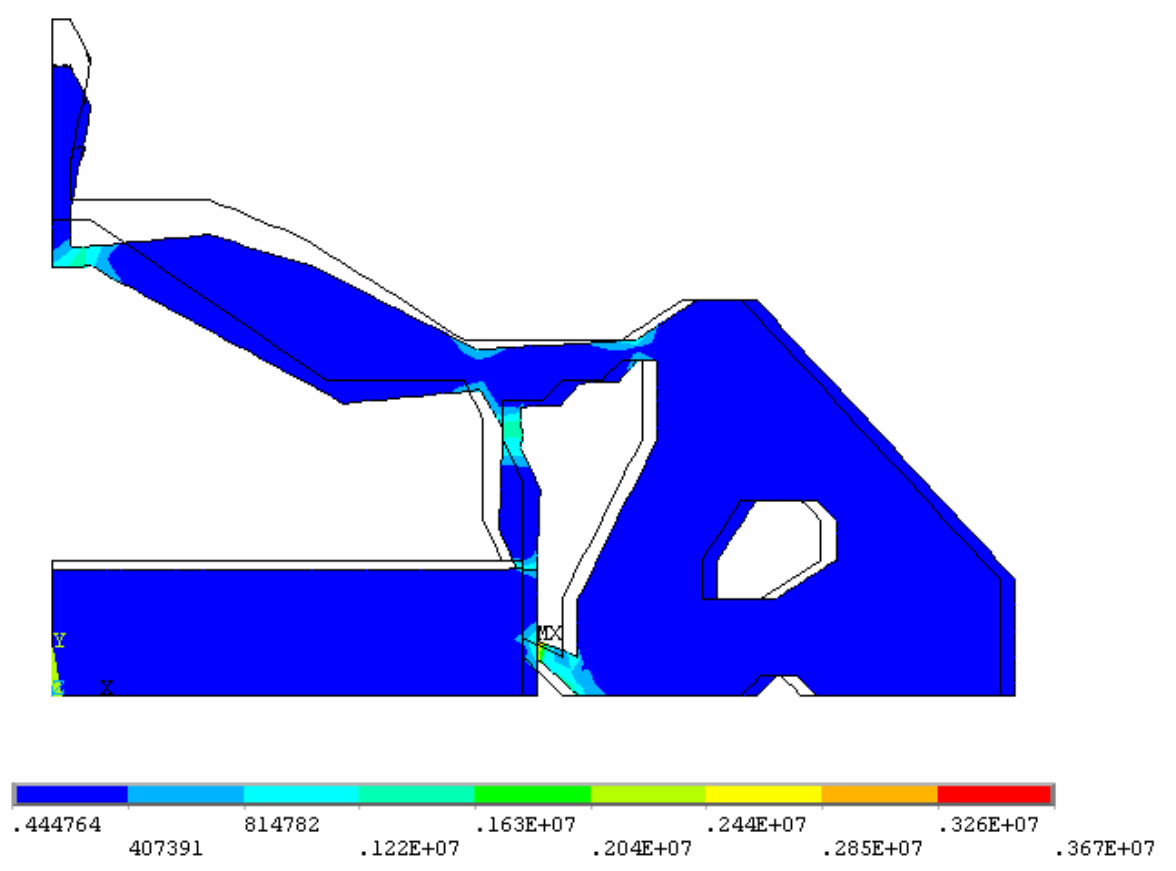

Figura 6.39 - Distribuição de tensões mecânicas (Von-Mises), unidades em $P a$. 


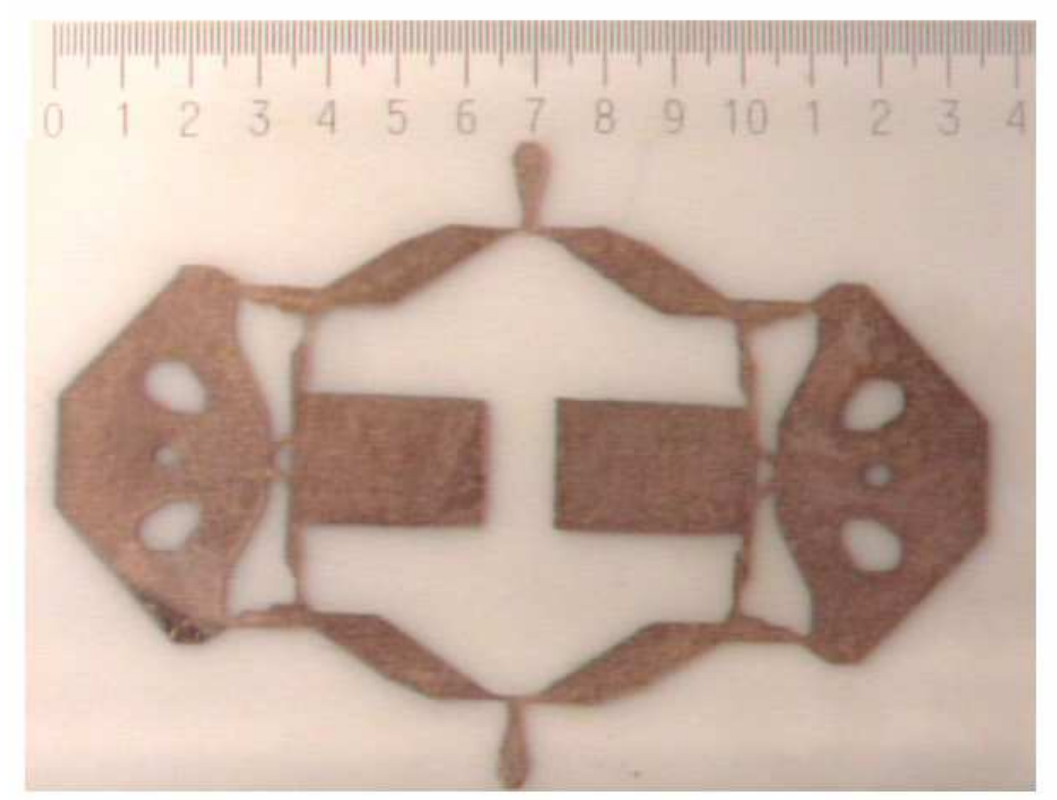

Figura 6.40 - Protótipo do atuador (escala, 1 divisão = 0,1 $\mathrm{mm}$ ).

\section{Exemplo 2}

A Figura 5.41 mostra o domínio inicial de projeto do atuador piezelétrico flextensional, onde devido à simetria do atuador (em relação à direção horizontal) somente 1/2 do domínio é apresentado. Os pontos de aplicação das cargas elétricas, do deslocamento desejado e das restrições de deslocamentos também são mostrados na mesma figura. O domínio é discretizado numa malha com 2200 elementos finitos.

A Fig. 5.42 ilustra a configuração da topologia ótima obtida, através do APFLEX. Analisando a deformada da topologia ótima (Fig. 6.43), percebe-se que o objetivo deste atuador é prender ou agarrar um determinado objeto durante a atuação, como especificado durante o projeto. Mas, como este atuador possui duas piezocerâmicas, ele apresenta uma amplificação da ordem de 15 vezes, na Tab 6.4 estão os valores dos deslocamentos obtidos para o ponto de atuação. Como no exemplo anterior, verifica-se que há poucos pontos de concentração de tensões, como observado na Fig. 6.44. O protótipo fabricado do atuador é mostrado na Fig. 6.45. 


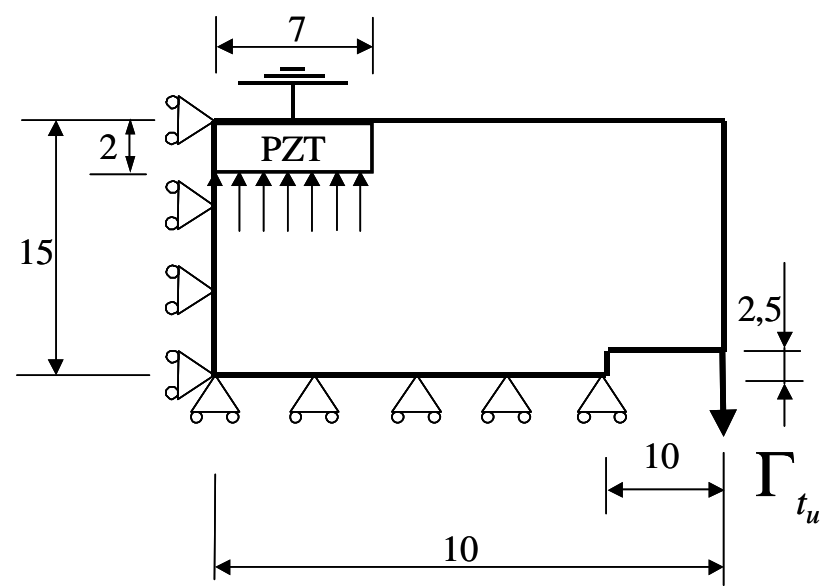

Figura 6.41 - Domínio inicial do atuador.

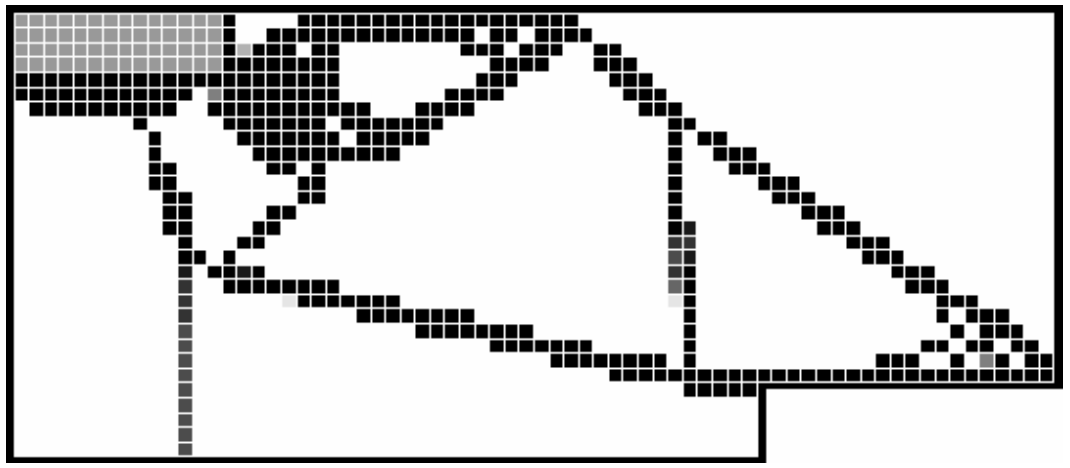

Figura 6.42 - Configuração da topologia ótima. 


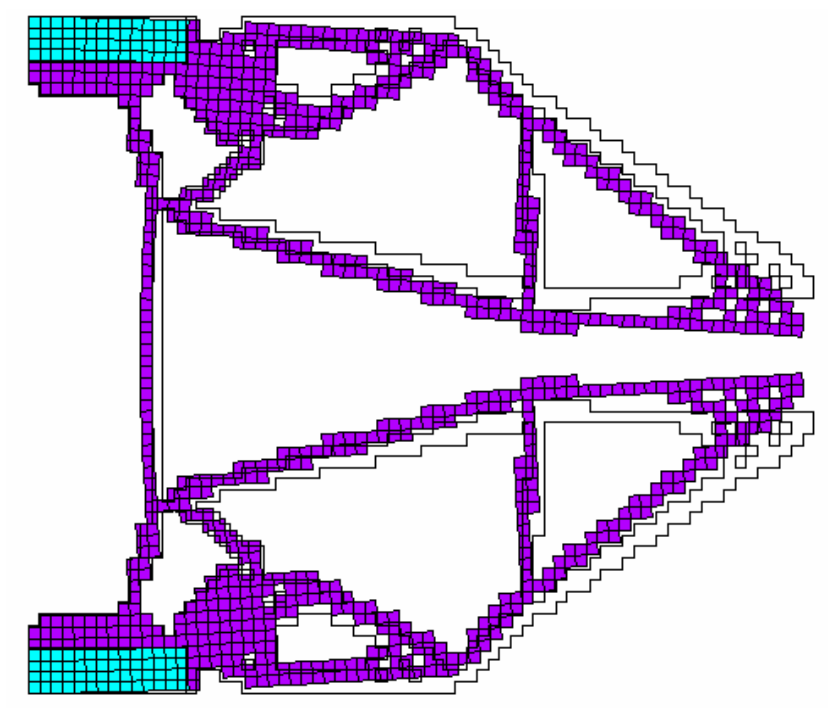

Figura 6.43 - Deformada da topologia ótima.
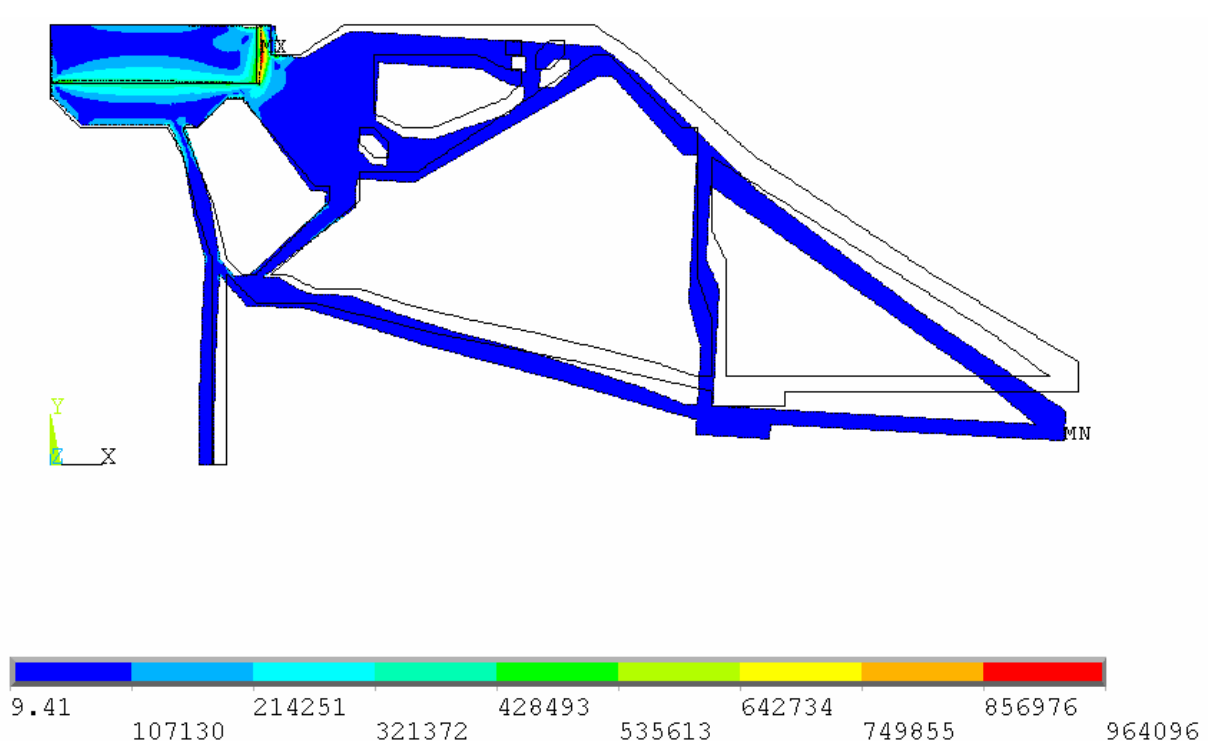

Figura 6.44 - Distribuição de tensões mecânicas (Von-Mises), unidades em $P a$. 


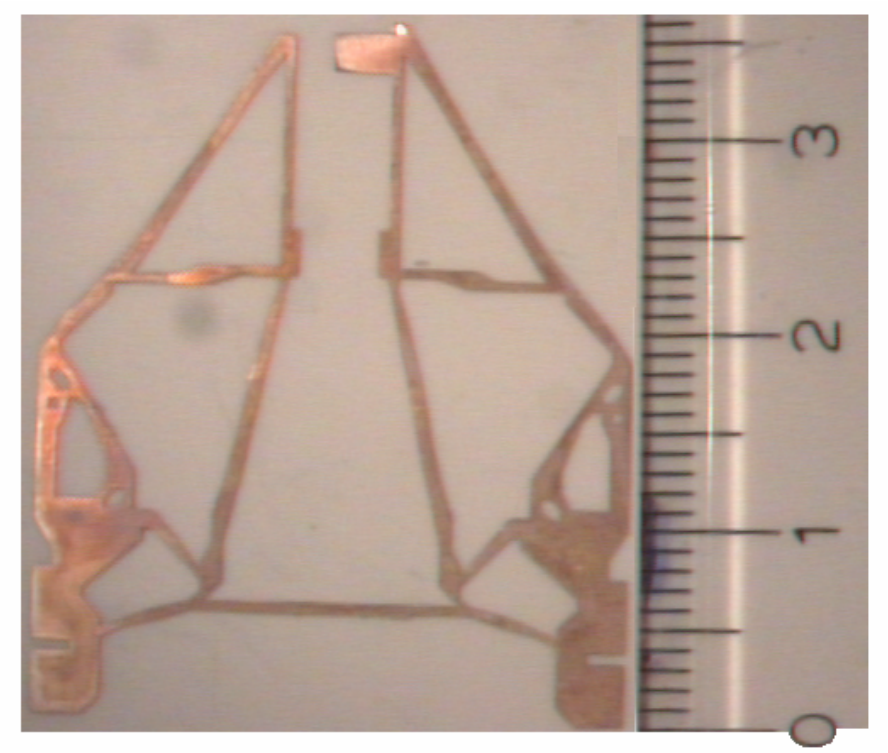

Figura 6.45 - Protótipo do atuador (escala, 1 divisão = 1,0mm).

\section{Exemplo 3}

A Figura 6.46 mostra o domínio inicial de projeto do atuador piezelétrico flextensional (CARROZZA et al., 1998), onde devido à simetria do atuador (em relação à direção vertical) somente $1 / 2$ do domínio é apresentado. Os pontos de aplicação das cargas elétricas, do deslocamento desejado e das restrições de deslocamentos também são mostrados na mesma figura. $\mathrm{O}$ domínio é discretizado numa malha com 2000 elementos finitos. O objetivo deste atuador piezelétrico flextensional é obter um microgarra para manipulação de células biológicas. No entanto, o projeto inicial desenvolvido por Carrozza et al. (1998) foi baseado na intuição física do problema. Os próximos resultados tem como objetivo projetar este atuador considerando condições de projeto semelhantes às empregadas por Carrozza et al. (1998).

A configuração da topologia ótima obtida é mostrada na Fig. 6.47. Na seqüência, as Fig. 6.48 e 6.49 ilustram, a verificação obtida através do ANSYS da topologia deformada e o campo de tensões de Von-Mises para a topologia 
interpretada, respectivamente. Na Tab. 6.4 é mostrado o deslocamento máximo na direção de atuação desejado para a topologia interpretada e na Fig. 6.50, o protótipo do atuador fabricado.

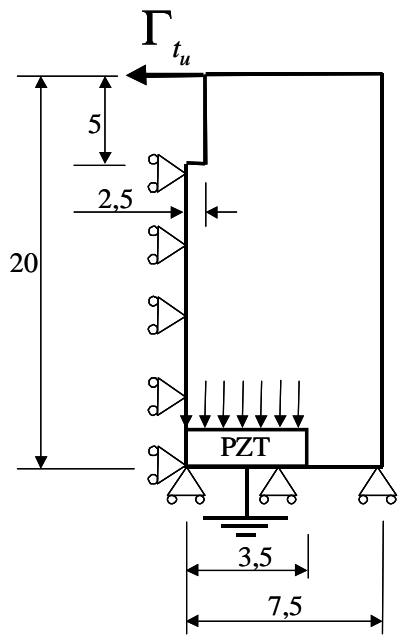

Figura 6.46 - Domínio inicial do atuador.

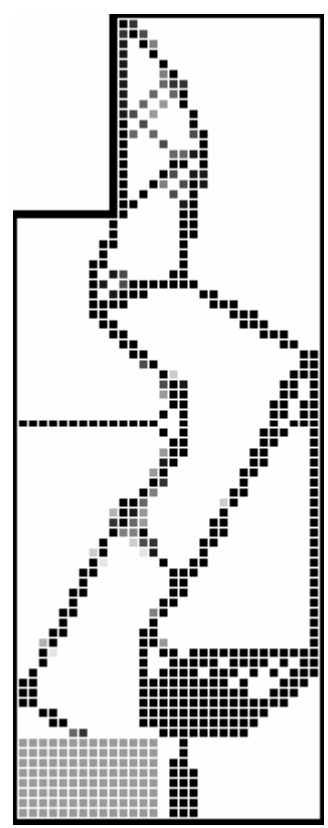

Figura 6.47 - Configuração da topologia ótima. 


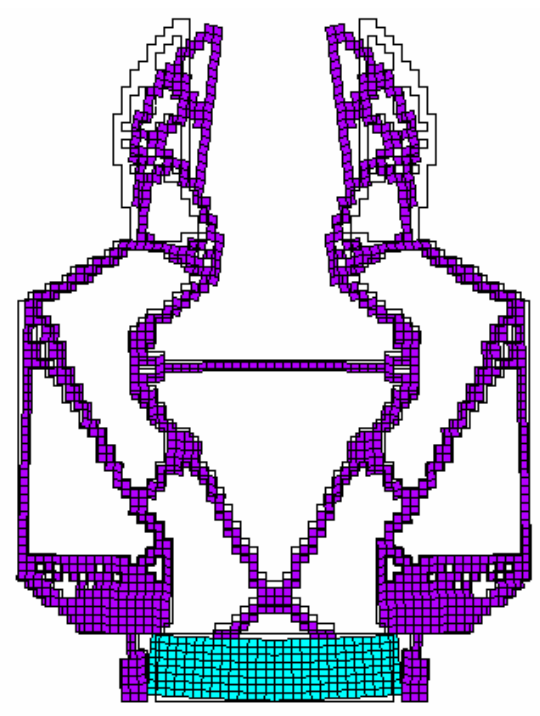

Figura 6.48 - Deformada da topologia ótima.
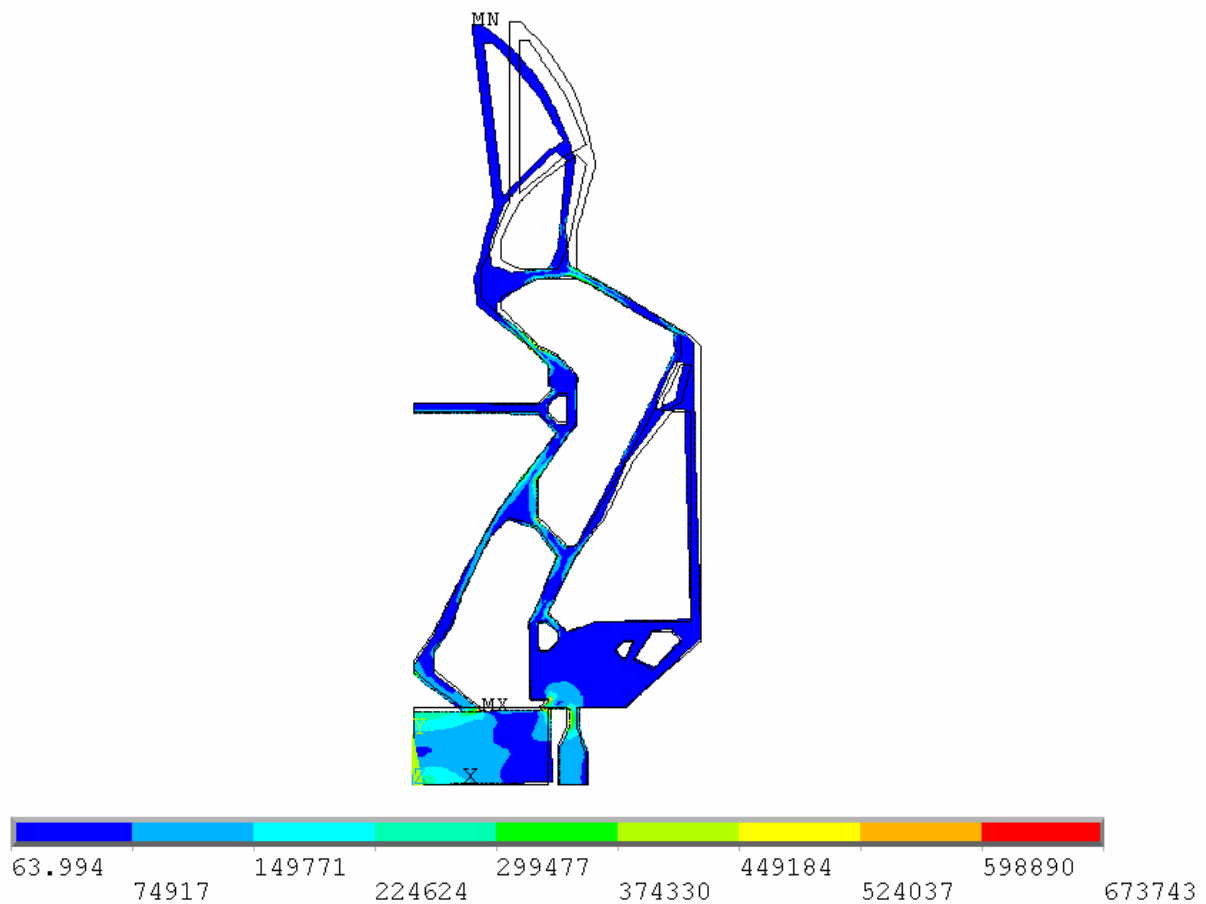

Figura 6.49 - Distribuição de tensões mecânicas (Von-Mises), unidades em Pa. 


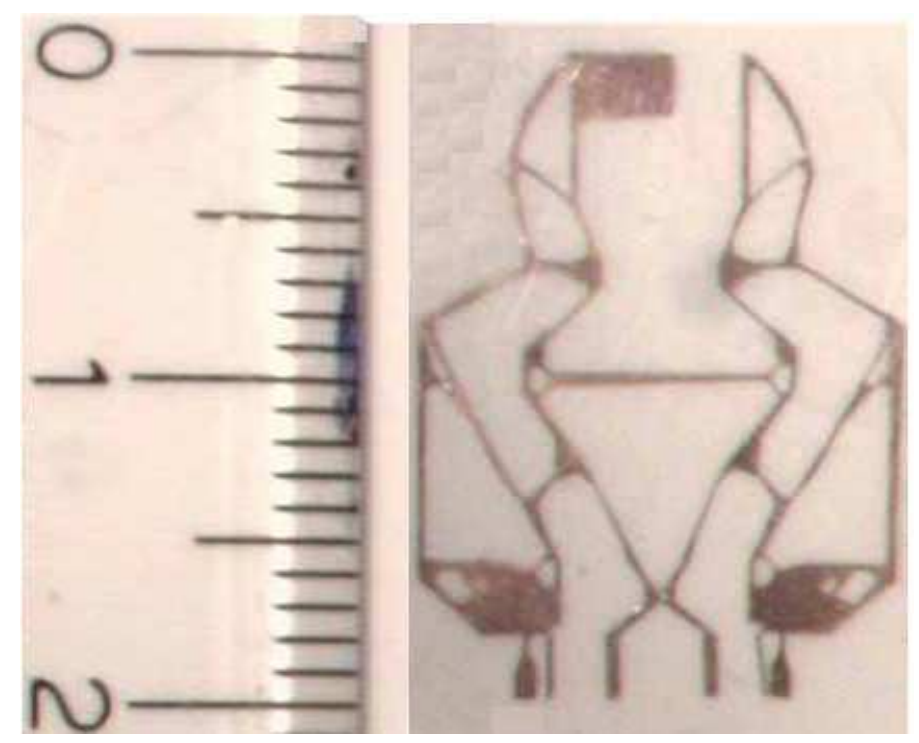

Figura 6.50 - Protótipo do atuador (escala, 1 divisão = 1,0mm).

\section{Exemplo 4}

A Figura 6.51 mostra o domínio inicial de projeto do atuador piezelétrico flextensional (FUKUDA et al., 1995), onde devido a simetria do atuador (em relação à direção horizontal) somente metade do domínio é apresentado. Os pontos de aplicação das cargas elétricas, do deslocamento desejado e das restrições de deslocamentos também são mostrados na mesma figura. O domínio é discretizado numa malha com 2509 elementos finitos. O objetivo deste atuador piezelétrico flextensional é obter um microrobô nadador para aplicação em inspeção de tubos, ou seja, deseja-se que os movimentos da estrutura flexível produzidos pela piezocerâmica possam gerar propulsão no microrobô, dando ao atuador mobilidade nas paredes do tubo. No entanto, o projeto inicial desenvolvido por Fukuda et al. (1995) foi baseado em equações de dinâmica de mecanismos. Os próximos resultados têm como objetivo projetar um atuador semelhante geometricamente, que considera a direção $y$ como a direção de atuação e as demais condições de projeto semelhantes às empregadas por Fukuda et al. (1995), no qual, considera a direção $x$ como a direção de atuação e possui duas piezocerâmicas. 
Dessa forma, na Fig. 6.52 ilustra a configuração da topologia ótima obtida. $\mathrm{Na}$ seqüência, a Fig. 6.53 ilustra a verificação, obtida através do ANSYS, da topologia deformada e na Fig. 6.54 o campo de tensões de Von-Mises para a topologia interpretada. A Tab. 6.4 indica o valor dos deslocamentos no ponto de atuação para a topologia ótima interpretada. A última figura deste item ilustra o protótipo do atuador (ver Fig. 6.55).

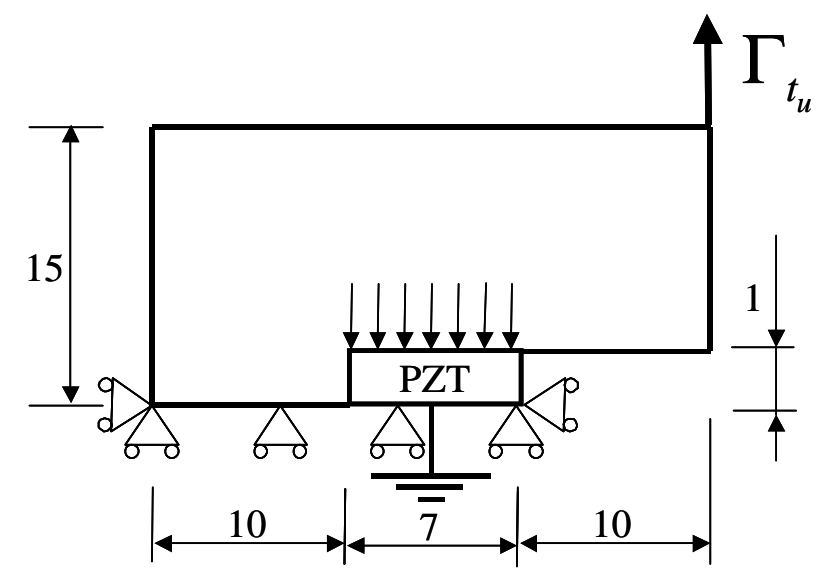

Figura 6.51 - Domínio inicial do atuador.

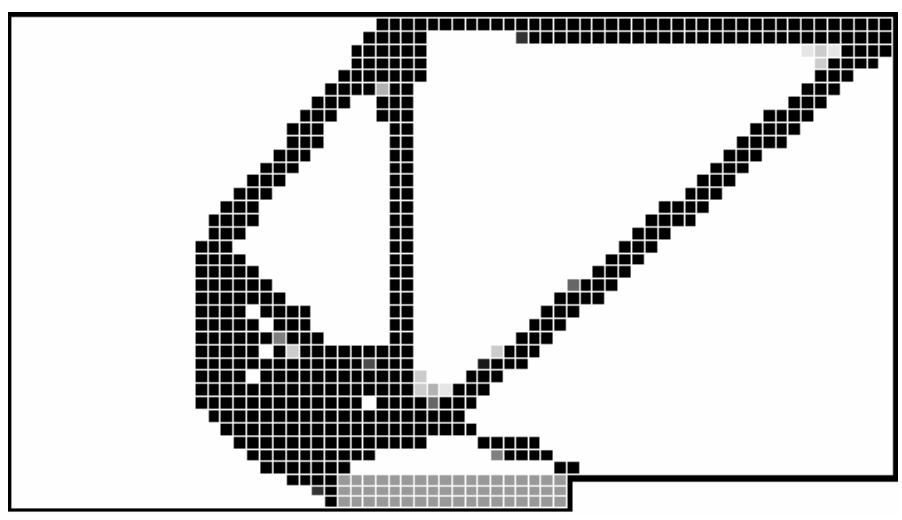

Figura 6.52 - Configuração da topologia ótima. 


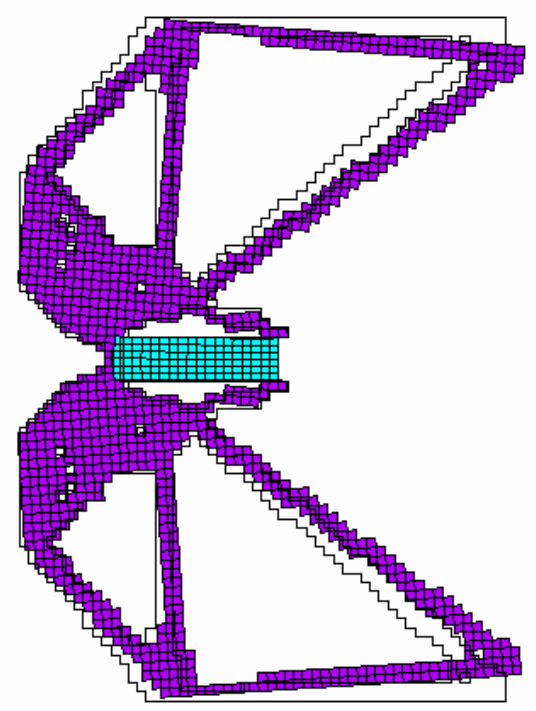

Figura 6.53 - Deformada da topologia ótima.
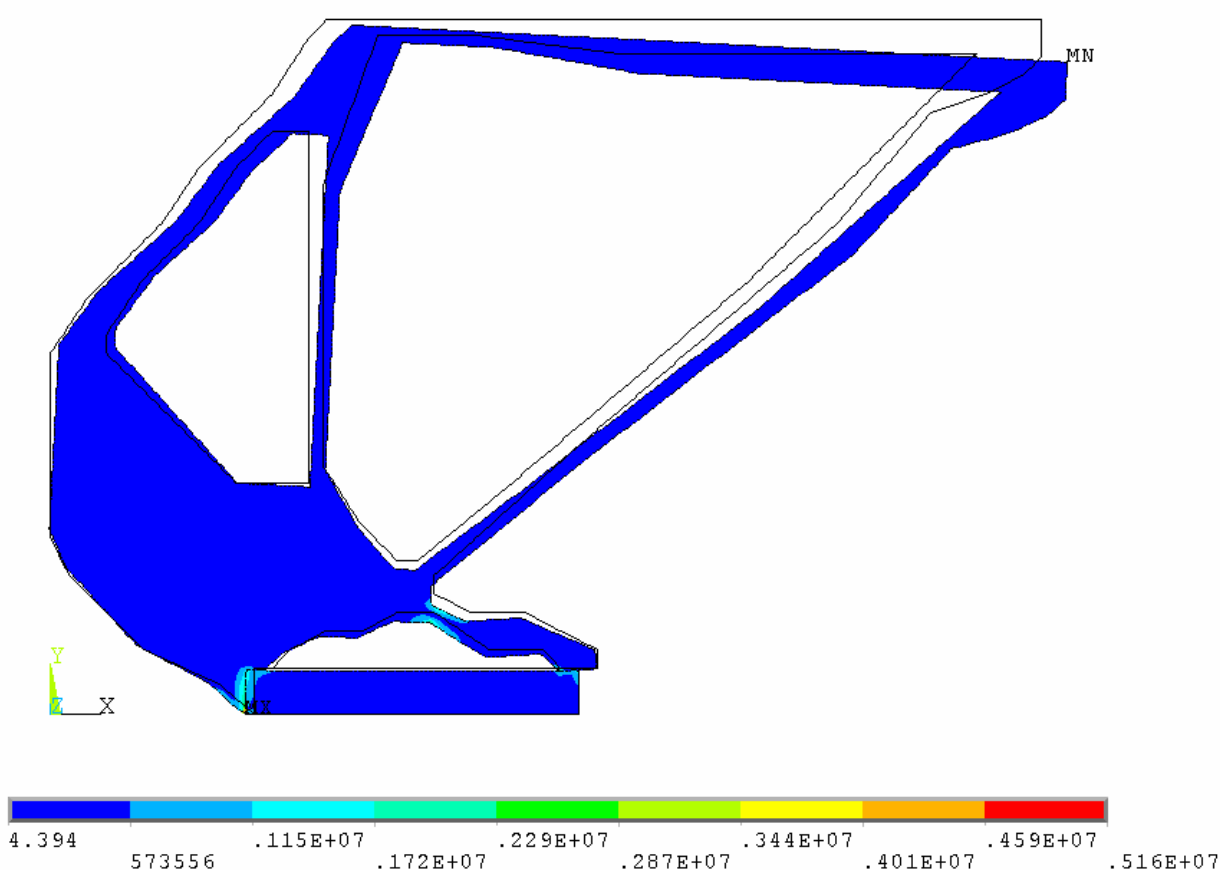

Figura 6.54 - Distribuição de tensões mecânicas (Von-Mises), unidades em $P a$. 


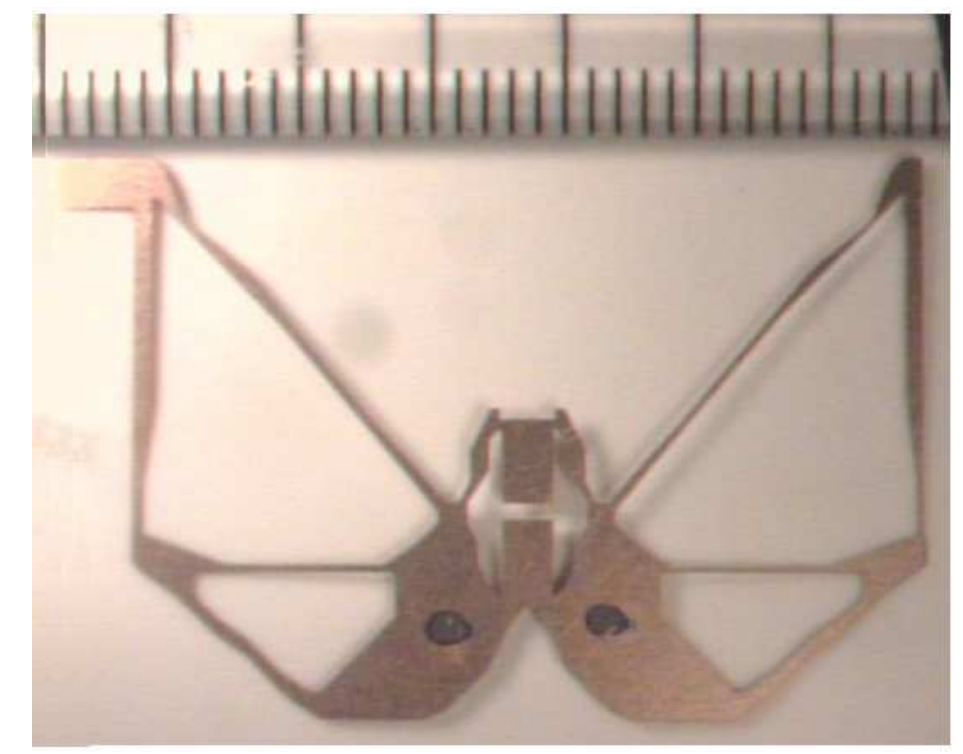

Figura 6.55 - Protótipo do atuador (escala, 1 divisão = 1,0 $\mathrm{mm}$ ).

\begin{tabular}{|c|c|c|}
\hline & Direção $x\left(10^{-7} \mathrm{~m}\right)$ & Direção $y\left(10^{-7} \mathrm{~m}\right)$ \\
\hline Exemplo 1 & 0,04 & 1,90 \\
\hline Exemplo 2 & 2,10 & 6,70 \\
\hline Exemplo 3 & 3,34 & 0,47 \\
\hline Exemplo 4 & 3,36 & 5,50 \\
\hline
\end{tabular}

Tabela 6.4 - Valores dos deslocamentos para o ponto de atuação.

\subsection{Verificações dos resultados obtidos no Exemplo 4}

Como modelos 2D não representam com exatidão o problema físico, é necessário construir um modelo 3D para analisar os deslocamentos fora do plano do 
atuador e a influencia da espessura da piezocerâmica. As características geométricas deste modelo 3D são: espessura do cobre é igual à $60 \mu \mathrm{m}$ e a dimensão da piezocerâmica é $7 \times 2 \times 1 \mathrm{~mm}^{3}$. Com estes dados geométricos, foram construídos modelos 3D a partir do protótipo fabricado (Fig. 6.55) para verificar o comportamento do atuador durante a deformação através do software ANSYS. Utilizando uma câmera CCD acoplada a um microscópio ("Probe Station") com uma ampliação de 1000 vezes (resolução de $1 \mu \mathrm{m}$ ) foi possível visualizar a deformação do atuador aplicando uma tensão 100 volts. A grandeza dos deslocamentos para uma tensão de 100 volts é da ordem de $0,50 \mu m$, como verificado no item 6.5.2 Tab. 6.4, onde os resultados foram obtidos usando o software ANSYS considerando modelos 2D.

As figuras abaixo (Fig 6.56 e 6.57) ilustram a deformada e a tensão de VonMises, respectivamente. Os valores dos deslocamentos nas direções $x, y$ e $z(x-$ direção de atuação; $y$ - direção fora do plano de atuação e $z$ - direção secundária) são dados na Tab. 6.5 (Modelo 3D)

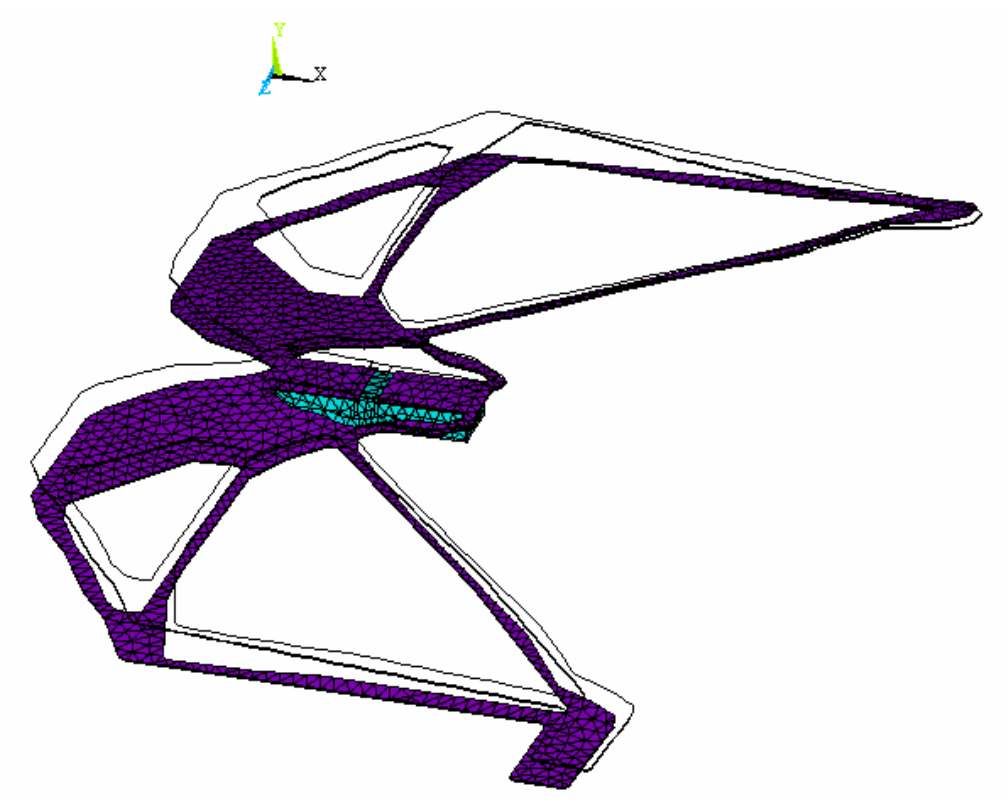

Figura 6.56 - Deformada do modelo 3D. 


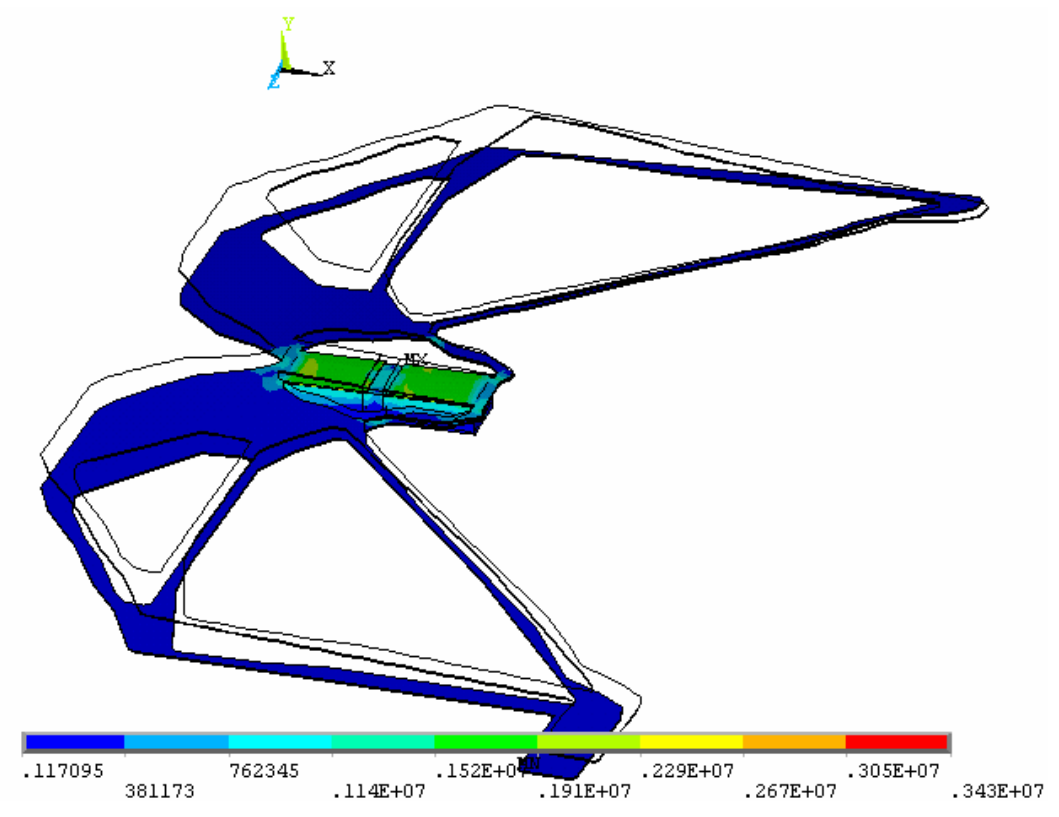

Figura 6.57 - Distribuição de tensões mecânicas (Von-Mises), unidades em $P a$.

Os resultados analisados através das figuras acima e os apresentados na Tab. 6.5 , pretendem verificar o comportamento do atuador quando deformado utilizando um software de elementos finitos, ou seja, tendo um caráter qualitativo da deformação do atuador para as condições de projeto.

Percebe-se que há deslocamentos na direção y (deformação fora do plano) dá ordem de grandeza do deslocamento principal (ver Tab. 6.5 Modelo 3D), que surgem devido às tensões residuais (surgem durante o processo de fabricação), ao contato piezocerâmica / estrutura flexível gerar momento fletor e a flambagem. No entanto, comparando as tensões de Von-Mises da Fig. 6.54 com as da Fig. 6.57, notase que o valor das tensões de Von-Mises diminuíram devido ao contato distribuído na superfície entre a cerâmica e a estrutura flexível.

Uma sugestão para solucionar o problema da flambagem é aumentar a espessura da estrutura flexível, colando várias estruturas flexíveis com epóxi, como, um sanduíche de estruturas flexíveis. Desta forma, não seria necessário utilizar outro processo de fabricação.

A solução para contornar o problema do contato cerâmica / estrutura 
flexível é encaixar a cerâmica na estrutura flexível por interferência, de modo que as linhas médias de ambas estruturas coincidam, eliminando o momento fletor (ver Fig. 6.58). Através de simulações feitas no software ANSYS, percebe-se que há uma redução de 20 vezes nos deslocamentos máximos fora do plano, ver Tab. 6.5 (Modelo 3D A). Com este modelo, o contato entre a cerâmica e a estrutura flexível apresenta uma superfície de contato menor do que o modelo mostrado na Fig. 6.57, o que acaba gerando um pequeno aumento nos valores das tensões de Von-Mises, ver Fig. 6.58. A deformada é mostrada na Fig. 6.59.

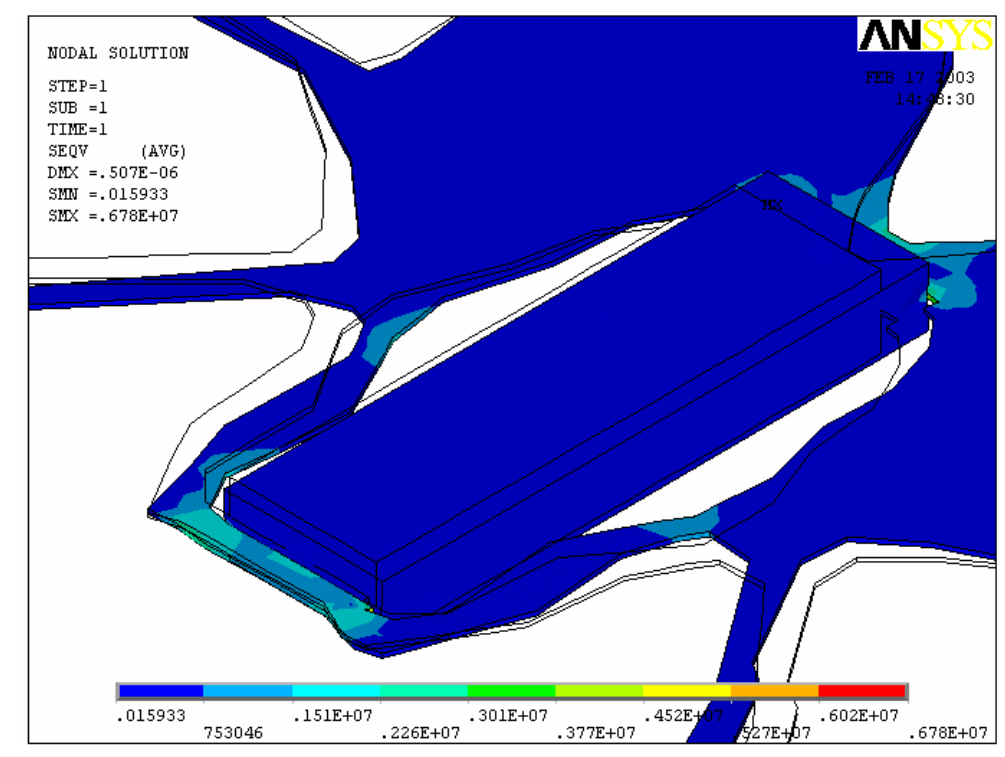

Figura 6.58 - Distribuição de tensões mecânicas (Von-Mises), unidades em $P a$. 


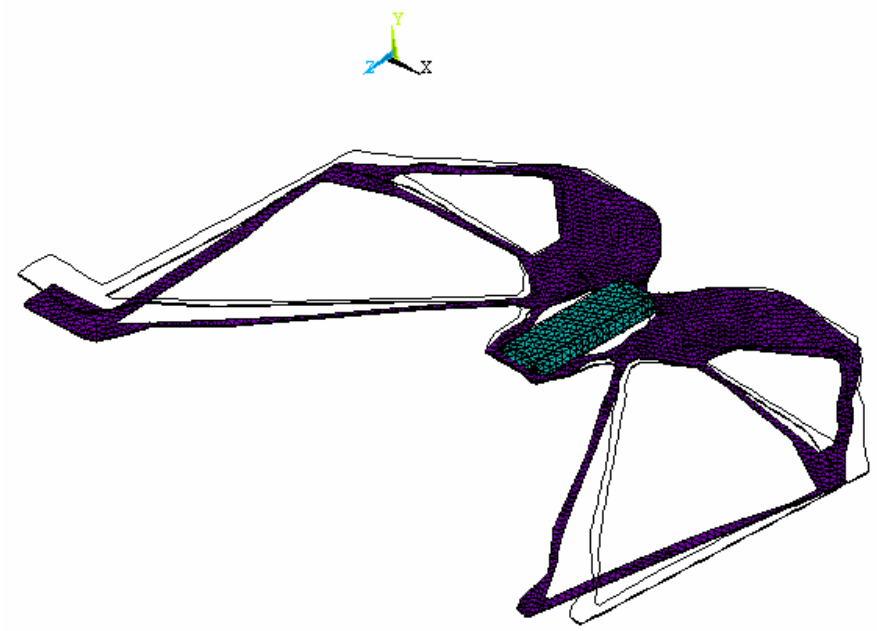

Figura 6.59 - Deformada do modelo 3D A.

\begin{tabular}{|c|c|c|c|}
\hline & Direção $x\left(10^{-7} m\right)$ & Direção $y\left(10^{-7} m\right)$ & Direção $z\left(10^{-7} m\right)$ \\
\hline Modelo 3D A & 4,51 & 0,379 & 2,83 \\
\hline Modelo 3D & 4,05 & 8,06 & 2,56 \\
\hline
\end{tabular}

Tabela 6.5 - Valores de deslocamentos para o ponto de atuação. 


\section{Capítulo 7: Conclusão}

\subsection{Conclusões e Observações}

Neste trabalho o método de Otimização Topológica, baseado no método das densidades (SIMP), foi implementado para projetar atuadores piezelétricos flextensionais. Esses atuadores piezelétricos têm grande aplicação em dispositivos de mecânica de precisão, na área biomédica e MEMS. O desenvolvimento do software foi feito em linguagem C. O software, denominado de APFLEX, utiliza a Programação Linear Seqüencial (PLS), que incorpora rotinas de programação matemática e método dos elementos finitos (MEF). Uma função multi-objetivo, que considera máxima flexibilidade e mínima rigidez, foi definida para o problema de otimização sujeito a restrição de volume de material da estrutura. Com o APFLEX, conseguimos obter bons resultados que consolidam a potencialidade do MOT aplicado ao projeto de atuadores piezelétricos flextensionais. Através da implementação de uma rotina de filtro espacial, tornou-se possível o controle dos parâmetros que influenciam na OT, como, as "escalas de cinza", a "instabilidade xadrez" e a dependência ao aumento da discretização do domínio inicial.

O MEF incorpora as seguintes rotinas: duas formulações distintas de elemento (elemento piezelétrico e não-piezelétrico), uma rotina SKYLINE e uma rotina de Gauss (BATHE, 1996). O APFLEX conta também com uma rotina que desenvolve a integração numérica da formulação genérica do MEF para o elemento quadrilátero isoparamétrico de 4 nós, em estado plano de tensão ou deformação. $\mathrm{O}$ elemento finito de 4 nós é um dos mais utilizados nos trabalhos da literatura que aplicam o método de Otimização Topológica, pelo fato do baixo custo computacional exigido em relação aos elementos de alta ordem.

Outra característica importante é a eficiência computacional ganha com a implementação da rotina de programação linear DSPLP, que produz a otimização de milhares de variáveis de projeto numa velocidade consideravelmente alta. 
Dessa forma, através do APFLEX é perfeitamente possível projetar sistematicamente atuadores piezelétricos flextensionais para obter atuadores bidimensionais complexos e com movimentos diversos, que podem ser aplicados em diversas áreas. Com isto, foi possível projetar e fabricar atuadores piezelétricos flextensionais com aplicações diversas numa mesoescala, usando corrosão química através do método de litografia, tecnologia disponível no LNLS em Campinas. O material utilizado na fabricação foi o cobre, com espessuras de 60, 100 e $150 \mu \mathrm{m}$. Usando um microscópio com uma ampliação de 1000 vezes (definição de $1 \mu \mathrm{m}$ ) foi possível visualizar a deformação de um protótipo fabricado. As simulações foram realizadas no software ANSYS, considerando modelos 2D e 3D, para analisar o comportamento de sua deformação, quando atuado. Desta forma, percebemos que o atuador gera grandes deslocamentos fora do plano de atuação, devido às tensões residuais, ao tipo de contato cerâmica / estrutura flexível e a flambagem.

\subsection{Considerações Finais e Trabalhos Futuros}

Cumprido o objetivo deste trabalho de mestrado, espera-se ter contribuído com um software genérico e sistemático para o projeto de atuadores piezelétricos flextensionais usando o método de otimização topológica, que pode ser aplicado por cientistas de outras áreas. Com a existência deste software será possível uma maior divulgação da tecnologia de atuadores piezelétricos flextensionais entre o meio acadêmico, alunos de graduação e pós-graduação em engenharia. Como trabalho futuro, uma rotina para eliminar os problemas das juntas na topologia ótima pode ser implementado (POULSEN, 2002). Outra sugestão é desenvolver uma nova versão do software, implementando o conceito de multiflexibilidade, para projetar atuadores com múltiplas entradas e respostas. Além disso, outras funções objetivo e domínios iniciais podem ser considerados e implementados dependendo da aplicação específica desejada para o atuador piezelétrico flextensional. 


\section{ANEXO A: Descrição da utilização e funcionamento do software}

O objetivo deste anexo é apresentar um procedimento básico para a utilização e funcionamento do software de Otimização Topológica (APFLEX) implementado neste trabalho de mestrado. Conhecendo-se o domínio estendido fixo (domínio inicial) para o projeto do atuador piezelétrico flextensional, o usuário deverá seguir 3 etapas distintas para obtenção da topologia otimizada e, conseqüentemente, a forma final do atuador piezelétrico. A Fig. A.1 apresenta o fluxograma básico da estrutura de utilização do APFLEX. A primeira etapa é o pré-processamento, através do ANSYS, para geração dos dados iniciais; a segunda etapa é o processamento dos dados iniciais, através do APFLEX, para obtenção da topologia otimizada; e a terceira etapa é o pós-processamento para visualização, interpretação e análise da topologia obtida. Essas três etapas serão detalhadas a seguir.

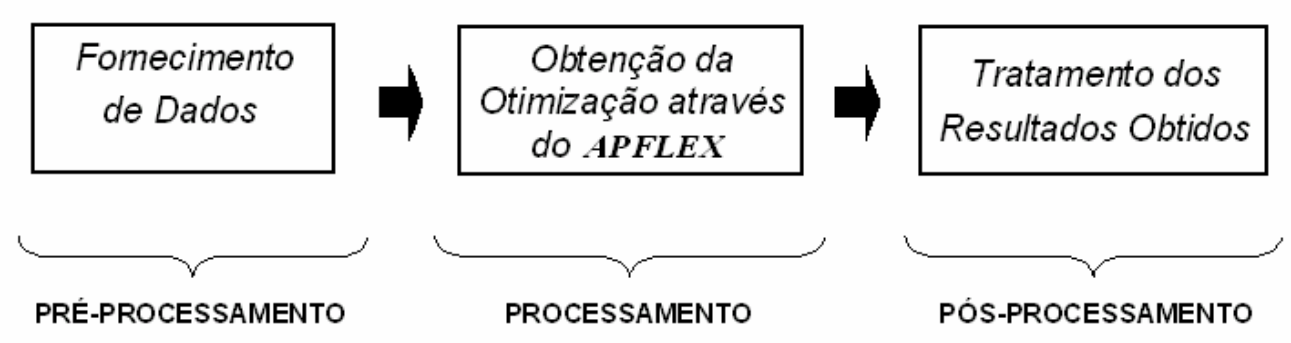

Figura A.1 - Fluxo básico da estrutura de utilização do APFLEX.

\section{A.1. Pré-processamento}

O pré-processamento é realizado no ANSYS (software comercial de elementos finitos) e consiste em se criar o modelo do atuador piezelétrico que se 
deseja obter, fornecendo: a geometria do domínio inicial; informações sobre as características da malha de elementos finitos, como, por exemplo, número de nós, número de elementos, as coordenadas de cada nó, a conectividade dos elementos, o tipo de elemento e material para cada elemento; e as condições de carregamento elétricos e mecânicos aplicados nos nós e também os graus de liberdade restringidos, tanto elétricos quanto mecânicos. Basicamente, o usuário utiliza no ANSYS somente os recursos de $\mathrm{CAD}$ e gerador de malhas para criar um arquivo texto $(<$ nome do arquivo>.txt), no formato padrão do ANSYS (ASCII). O APFLEX necessita da informação de três carregamentos diferentes (veja capítulo 4), a qual deve estar contida no mesmo arquivo texto. É importante observar que o usuário deve gerar somente uma malha, a qual deve conter inicialmente as condições de contorno e aplicações de forças do $1^{\circ}$. e $2^{\circ}$. caso de carregamento. Com isso, cria-se um arquivo texto do tipo $D B$ data através da função CDWRITE do ANSYS. Após isso, o usuário deve utilizar a mesma malha, substituindo as condições de contorno e aplicação de forças pelo $3^{\circ}$. caso de carregamento e criar um outro arquivo texto Loads através da função CDWRITE do ANSYS. O primeiro arquivo ( $D B$ data) é completo e contém todas as informações necessárias sobre a malha de elementos finitos, já o segundo arquivo contém somente a informação sobre restrição de nós e aplicação de forças do $3^{\circ}$. caso de carregamento. Assim, é necessário unir o conteúdo dos dois arquivos e incluir alguns dados complementares que são inseridos manualmente, são eles o coeficiente w (WEIGH), restrição de volume (VFRAC), fator de penalidade $p$ (PENAL), restrições laterais das variáveis de projeto (XMIN0 e XMAX0) e o valor inicial das variáveis de projeto (XINIO).

\section{A.2. Processamento com o APFLEX}

O processamento para obtenção da topologia ótima aplicada ao projeto de atuadores piezelétricos flextensionais, é realizado no APFLEX. O fluxograma da Fig. A.2 mostra as atividades desenvolvidas pelo software para obtenção da topologia ótima. 


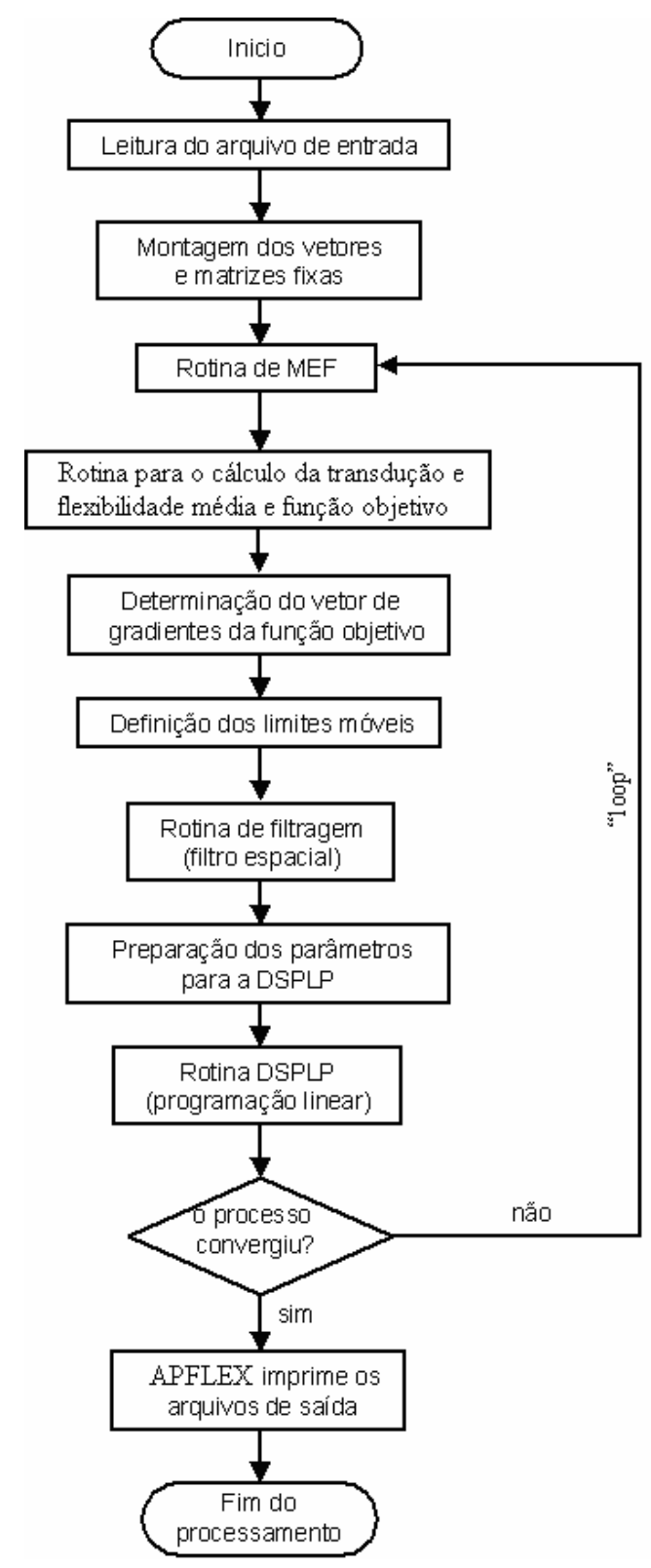

Figura A.2 - Fluxo geral de funcionamento do APFLEX.

Desta forma é feita a compilação do arquivo fonte em qualquer compilador de linguagem $\mathrm{C}$. A escolha do compilador fica a critério do usuário, neste trabalho foi utilizado o compilador do sistema operacional Linux que, para este trabalho se demonstrou um sistema mais rápido e com melhor gerenciamento de memória, para rodar softwares numéricos de grande porte. No passo seguinte, basta o usuário 
mandar processar o arquivo executável gerado pelo compilador. A partir desse ponto, o processamento é automático pelo software.

A rotina de MEF constituída de duas formulações distintas, uma piezelétrica e outra não-piezelétrica executa um "loop" interno de $M$ voltas (sendo $M$ o número total de elementos) que, em cada volta, identifica o tipo de elemento e constrói a matriz de rigidez local do elemento especificado e posiciona os valores dessa matriz local na matriz de rigidez global, de acordo com a conectividade de cada elemento da malha. A matriz de rigidez do elemento é construída através de uma rotina de integração numérica de Gauss-Legendre (2x2 pontos) para a Eq. (3.47) (capítulo 3). A matriz de rigidez global é montada de maneira que sejam desconsideradas as linhas e colunas referentes aos deslocamentos nulos (nós fixos), isto reduz o tamanho da matriz e, conseqüentemente, o tempo de processamento. A Fig. A.3 mostra o fluxograma específico da rotina de MEF. Após o "loop", a rotina de MEF armazena a matriz de rigidez global "cheia" no formato "skyline". E para cada caso de carregamento $\left(1^{\circ} ., 2^{\circ}\right.$. e $3^{\circ}$.), a rotina obtém os deslocamentos nodais correspondentes, resolvendo o sistema de equações de equilíbrio de cada caso (Eq. 3.49), através de uma rotina de eliminação de Gauss (que também é válida para matrizes indefinidas). Os vetores de deslocamentos nodais (configuração deformada de cada caso de carregamento) são obtidos a partir do vetor de densidades dos elementos (variáveis de projeto), atualizado a cada iteração do processo de otimização. Uma vez obtidos esses vetores, o APFLEX calcula o valor da transdução média e flexibilidade média e, conseqüentemente, o valor da função objetivo e suas derivadas (gradientes). 


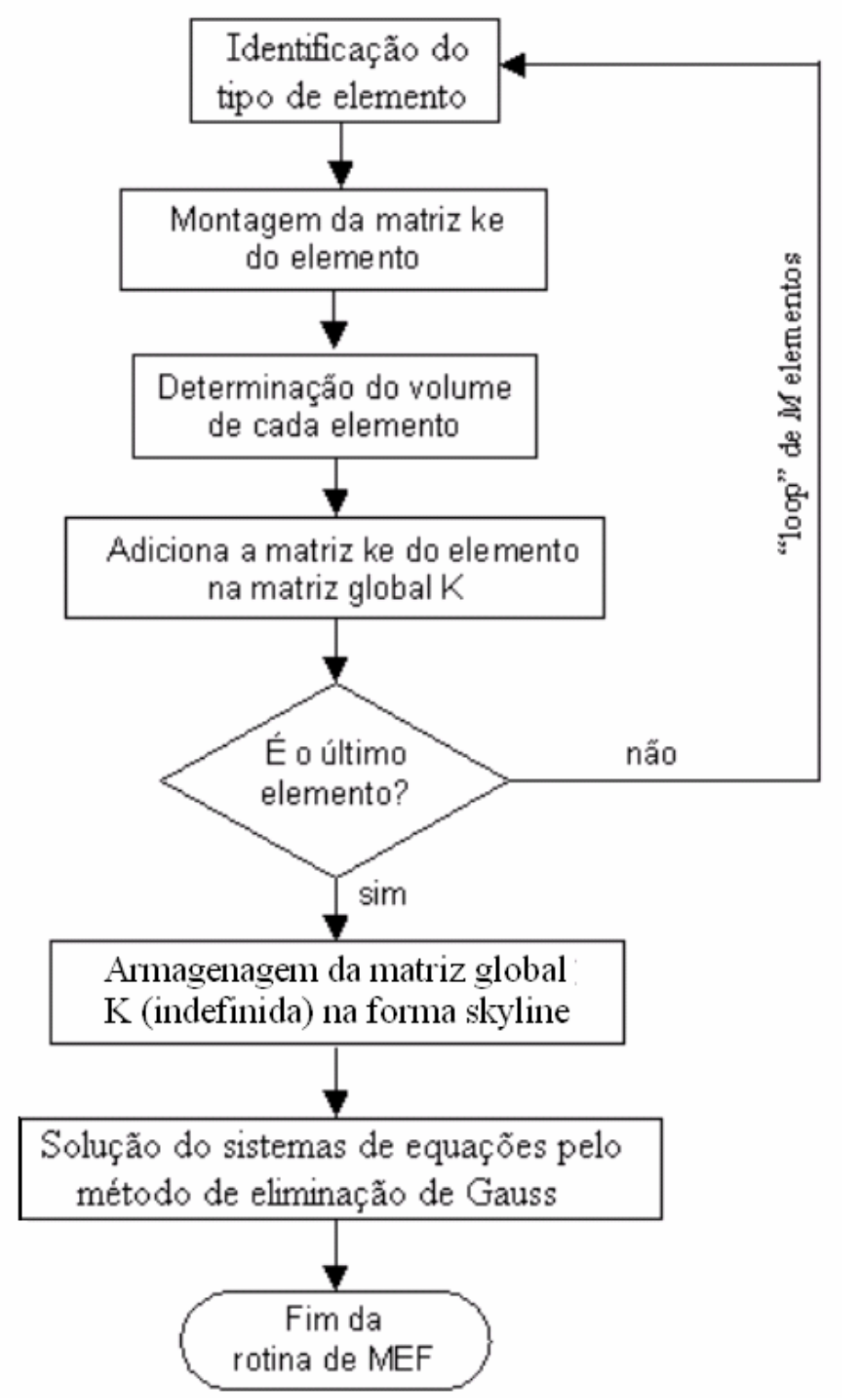

Figura A.3 - Fluxo da rotina de MEF.

\section{A.3. Pós-processamento}

O pós-processamento é realizado no ANSYS. Depois de terminado o processo de otimização, o APFLEX gera dois arquivos texto, em formato padrão lido pelo ANSYS, um deles (otANSYS.txt) contém a informação necessária para visualizar a configuração ótima da topologia e o outro (otmlhANSYS . txt) contém a 
informação para importar uma malha gerada a partir da distribuição ótima de densidades dos elementos.

$\mathrm{O}$ arquivo otANSYS.txt é formado a partir da rotina do APFLEX que escreve, em formato ASCII lido pelo ANSYS, um arquivo texto com as componentes dos vetores de coordenadas e de conectividade dos nós dos elementos, além do número da cor que é atribuído a cada elemento conforme o valor da densidade obtida para o mesmo na otimização. Para que o ANSYS interprete esse número, é fornecido para ele um arquivo do mapa de cores (criado a partir do utilitário CMAP do ANSYS), o qual contém um intervalo de dez cores em preto e branco. Assim as regiões de cor preta indicam um valor máximo para a densidade do elemento, a cor branca indica o valor mínimo e as tonalidades de cinza indicam os valores intermediários de densidades. O APFLEX permite gerar uma malha de elementos finitos no formato lido pelo ANSYS, através do arquivo otmlhANSYS.txt para posterior análise e verificação da topologia obtida. 


\title{
REFERÊNCIAS BIBLIOGRÁFICAS
}

\author{
ALLIK, H.; Hughes, T. J. R., 1970, "FINITE ELEMENT METHOD FOR \\ PIEZOELECTRIC VIBRATION", International Journal for Numerical \\ Methods in engineering, Vol 2, pp. 151-157.
}

ANANTHASURESH, G. K.; Kota S. e Gianchandani, Y., 1994, “A Methodical Approach to the Design of Compliant Micromechanisms", Solid-state Sensor and Actuator Workshop, pp. 189-192.

ANANTHASURESH, G. K.; Kota S., 1995, "Designing Compliant Mechanisms", ASME Mechanical Engineering, November, 93-96.

AULD, B. A., 1990, “Acoustic Fields and Waves in Solids", v. I. Florida - EUA: Robert E. Krieger Publishing Company, $2^{a}$ ed..

BATHE, K.J., 1996, "Finite Elements Procedures", Prentice Hall, New Jersey.

BARBER, J. M., 1992, "Elasticity", Kluwer Academic, Dordrecht.

BENDSØE, M. P., Kikuchi, N., 1988, “Generating Optimal Topologies in Structural Design Using a Homogenization Method". Computer Methods in Applied Mechanics and Engineering, 71, pp. 197-224.

BENDSØE, M., P., 1995, "Optimization of Structural Topology, Shape and Material", Springer - Verlag, New York. 
BENDSØE, M. P., 1989, "Optimal Shape Design as a Material Distribution Problem", Structural Optimization, 1, pp. 192-202.

BENDSØE, M.P., Rodrigues, H.C., 1991, "Integrated Topology and Boundary Shape Optimization of 2-D Solids", Computer Methods in Applied Mechanics and Engineering, vol. 87, pp 15-34.

BENDSØE M.P., Ben-Tal, A., e Zowe, J., 1994, “Optimization Methods for Truss Geometry and Topology Design”, Structural Optimization, 7, pp. 141-159.

BENDSØE, M., P., 1995, "Optimization of Structural Topology, Shape and Material", Springer - Verlag, New York.

BENDSØE, M. P., Sigmund, O., 1999, "Material Interpolations Schemes in Topology Optimization", Archive of Applied Mechanics, vol. 69, pp. 635-654.

BOURDIN, B., 2001, "Filters in Topology Optimization", International Journal for Numerical Methods in Engineering, 50, pp. 2143-2158.

BRISSAUD, M., 1991, "Characterization of Piezoceramics”, IEEE Transactions on Ultrasonics, Ferroeletrics and Frequency control, vol 38, no 6, pp. 603-617.

CANFIELD, S., Frecker, M., 2000, “Topology optimization of compliant mechanical amplifiers for piezoelectric actuators", Struct Multidisc Optim 20, pp. 269-279. 
CARDOSO, E.L., Fonseca, J.S.O., 1999, "Intermediate density reduction and Complexity Control in the Topology Optimization", 20th Iberian LatinAmerican Congress on Computational Methods in Engineering (CILAMCE'99), Brasil.

CARrozZA, M. C.; Dario, P.; Menciassi, A. e Fenu, A., 1998, "Manipulating Biological and Mechanical Micro-Objects Using LIGA-Microfabricated EndEffectors", IEEE Conf. On Robotics and Automation, pp. 1811-1816.

CHALLANDE, P., 1990, “Optimizing Ultrasonic Transducers Based on Piezelectric Composites Using a Finite-Element Method", IEEE Transactions on Ultrasonics, Ferroeletrics and Frequency control, vol 37, no 2, pp. 135-140.

CHEN, T. 1993, "Calculation of the Move Limits for The Sequential Linear Programming Method", International Journal of Numerical Methods in Engineering, Vol. 36, pp. 2661-2679.

CHENG, T. K.; Olhoff, N., 1981, “An Investigation Concerning Optimal Design of Solid Elastic Plates”, International Journal of Solids Structures, 17, pp. 305323.

CHENG, T. K.; Olhoff, N., 1982, "Regularized Formulation for Optimal Design of Axisymmetric Plates", International Journal of Solids Structures, 18-2, 153169.

DANTZIG, G.B., 1963, "Linear Programming and Extensions", Princeton, New Jersey. 
LIMA, C. R.; Silva, E. C. N, 2002, "Projeto de Mecanismos Flexíveis Usando o Método de Otimização Topológica", dissertação de mestrado, São Paulo Brasil, Escola Politécnica da USP.

DÍAZ, A.R., Bendsøe, M. P., 1992, "Shape Optimization of Structures for Multiple Loading Conditions Using Homogenization Method", Structural Optimization, 4, 17-22.

DÍAZ, A.R., Kikuchi, N., 1992, "Solution to Shape and Topology Eigenvalue Optimization Problems Using a Homogenization Method", International Journal for Numerical Methods in Engineering, 35, pp. 1487-1502.

DÍAZ, A., Sigmund, O., 1995, "Checkerboard Patterns in Layout Optimization", Structural Optimization, 10, pp. 40-45.

DOGAN, A.; Yoshikawa, S.; Uchino, K. e Newnham, R. E., 1994, "The effect of Geometry on the Characterisitcs of the Moonie Transducer and Reliability Issue", IEEE Ultrasonics Symposim, pp. 935-939.

DOGAN, A.; Uchino, K.; Newnham, R. E., 1997, "Composite Piezoelectric Transducer with Truncated Conical Endcaps "Cymbal” ", IEEE Transactions on Ultrasonics Ferroelectrics and Frequency Control, Vol 44, No 3, pp. 597605.

FRECKER, M.I., Kikuchi, N., Kota, S., 1996, "Optimal Synthesis of Compliant Mechanisms to Satisfy Kinematics and Structural Requirements - Preliminary Results", Proceedings of ASME Design Engineering of Technical Conferences and Computers in Engineering Conference, Irvine, California, 96-DETCDAC1417. 
FRECKER, M.I.; Ananthasuresh, G. K.; Nishiwaki, S.; Kikuchi, N. e Kota, S., 1997, “Topology Synthesis of Compliant Mechanisms Using Multi-criteria Optimization”, Journal of Mechanical Design, Transaction of the ASME, 119, pp. 238-245.

FRECKER, M., Canfield, S., 2000, “Optimal Design and Experimental Validation of Compliant Mechanical Amplifiers for Piezoceramic Stack", Journal of Intelligent Material Systems and Structures, vol. 11 (5), pp. 360-369.

FUKUDA, T.; Kawamoto, A.; Arai, F e Matsuura, H.., 1995, "Steering Mechanism and Swimming Experiment of Micro Mobile Robot in Water", IEEE Conf. On Robotics and Automation, Vol 1, pp. 814-819.

FUJII, D., Chen, B.C., Kikuchi, N., 2001, "Composite Material Design of TwoDimensional Structures Using Homogenization Design Methods", International Journal for Numerical Methods in Engineering, 50, pp. 2031-2051.

FUKUSHIMA, J., Suzuki, K., Kikuchi, N., 1993, "Applications to Car Bodies: Generalized Layout Design of Three-Dimensional Shells", Optimization of Large Structural Systems, Ed. Rozvany, G.I.N, Doedrecht: Kluwer, pp. 177191.

GOODMAN, J., Kohn, R.V., Reyna, L., 1986, "Numerical Study of Relaxed Variational Problem from Optimal Design”, Computer Methods in Applied Mechanics and Engineering, 57, pp 107-127.

GOGOLA, M.; Goldfarb M., 1999, "Design of a PZT-Actuated Proportional Drum Brake", IEEE/ASME Transactions on Mechatronics, Vol 4, No 4, pp. 409-416. 
HABER, R.B., Jog, C.S., Bendsøe, M.P., 1996, "A New Approach to VariableTopology Shape Design Using a Constraint on Perimeter", Structural Optimization, Spring Verlag, 11, pp. 1-12.

HAFTKA, R.T., Gürdal, Z., Kamat, M.P., 1996, "Element of Structural Optimization", Kluwer Academic Publishers, Boston.

HASSANI, B., Hinton, E., 1998a, "A Review of Homogenization and Topology Optimization, I- Homogenization theory for media with periodic structure", Computer and Structures, 69, pp. 707-717.

HASSANI, B., Hinton, E., 1998b, "A Review of Homogenization and Topology Optimization, II- Analytical and numerical solution of homogenization equations", Computer and Structures, 69, pp. 719-738.

HASSANI, B., Hinton, E., 1998c, "A Review of Homogenization and Topology Optimization, III- Topology Optimization using optimality criteria”, Computer and Structures, 69, pp. 739-756.

HEMP, W.S., 1973, “Optimum Structures”, Clarebdon, Oxford.

HSU, Y., Hsu, M., Chen, C., 2001, "Interpreting Results from Topology Optimization Using Density Contours", Computers and Structures, 79, pp. 1049-1058.

IEEE, 1996, "Standard on Piezoeletricity", IEEE Transactions on Ultrasonics Ferroelectrics and Frequency Control, Vol 43, No 5, pp. 5-54. 
IKEDA, T., 1996, "Fundamentals of Piezoelectricity" Oxford - Inglaterra: Oxford University Press.

JOG, C.S., Haber, R.B., Bendsøe, M.P., 1994, “Topology Design with Optimized Self-Adaptive Materials" International Journal for Numerical Methods in Engineering, 37, pp. 1323-1350.

JOG., C.S., Haber, R.B., 1996, "Stability of Finite Element Models for Distributed Parameter Optimization and Topology Design", Computer Methods in Applied Mechanics and Engineering, 130, pp. 203-226.

JONSMANN, J., Sigmund, O., Bouwstra, S., 1999, "Compliant Thermal Microactuators", Sensors and Actuators, vol. 76, pp. 463-469.

JONSMANN, J.; Sigmund, O. e Bouwstra, S., 1999a, "Compliant Thermal Microactuators", Sensors and Actuators, vol. 76, pp. 463-469.

JONSMANN, J.; Sigmund, O. e Bouwstra, S., 1999b "Compliant Electro-thermal microactuators”. In MEAMS'99, pp. 588-593.

KIKUCHI, N., Hollister, S., Yoo, J., 1997, “A Concept of Image-based integrated CAE for production Engineering”, Proceedings of International Symposium on Optimization and Innovative Design in JSME, Tokio, Japan, pp. 75-90.

KINO, G. S., 1987, “Acoustic Waves: Devices, Imaging and Analog Signal Processing", Pretence-Hall - EUA. 
KOHN, R.V., Strang, G., 1986a, "Optimal Design in Elasticity and Plasticity", International Journal for Numerical Methods in Engineering, 22, pp. 183-188.

KOHN, R.V., Strang, G., 1986b, "Optimal Design and Relaxation of Variational Problems", Communications on Pure and Applied Mathematics, 39, pp. 1-25 (Part I), 139-182 (Part II), 353-377 (Part III).

KU, S. S.; Pinsopon, U.; Cetinkunt, S. e Nakajima, S. I., 2000, "Design, Fabrication, and Real-Time Neural Network Control of a Three-Degrees-of-Freedom Nanopositioner", IEEE/ASME Transactions on Mechatronics, Vol 5, No 3, pp. 273-283.

LARSEN, U. D.; Sigmund, O. e Bouwstra, S.,1997, "Design and Fabrication of Compliant Mechanisms and Material Structures with Negative Poisson's Ratio", Journal of Micro Electro Mechanical Systems "MEMS”, 6(2), pp. 99106.

LAU, G. K.; Du, H. e Lim, M. K., 2000, "Systematic Design of Displacementamplifying Mechanism for Piezoelectric Stacked Actuators Using Topology Optimization”, Smart Structures and Integrated Systems, Vol 3985, pp. 583591.

LERCH, R., 1990, "Simulation of Piezoelectric Devices by Two-and-ThreeDimensional Finite Elements", IEEE Transactions on Ultrasonics Ferroelectrics and Frequency Control, Vol 37, No 2, pp. 233-247.

LURIE, K.A., Federov, A.V., Cherkaev, A.V., 1982, "Regularization of Optimal Design Problems for Bar and Plates, Parts I and II", Journal of Optimizations and Theory and Applications, 37-4, pp. 499-521, 523-543. 
MA, Z.D, Kikuchi, N., Hagiwara, I., 1993, "Structural Topology and Shape Optimization for a Frequency Response Problem”, Computational Mechanics, 13, pp. 157-174.

MA, Z.D, Kikuchi, N., Cheng, H. C., 1995, "Topology Design for Vibration Structure”, Computer Methods in Applied Mechanics and Engineering, 121.14, pp. 259-280.

MADOU, M. J., 1997, “Fundamentals of Microfabrication”, CRC Press, EUA.

MEHTA, N. Y.; Haluck, R. S.; Frecker, M. I. e Snyder, A. J., 2002, 'Sequence and task analysis of instrument use in common laparoscopic procedures", Surgical Endoscopy-Ultrasound and Interventional Techniques 16 (2), pp 280-285.

MLEJNEK, H. P., 1992, "Some Aspects of the Genesis of Structures", Structural Optimization, vol. 5, pp. 64-69.

MURAT, F., Tartar, L., 1985, “optimality Conditions and homogenization”, Eds. Marino, A., Modica L., Spagnolo, S., and Degiovanni, M., Nonlinear Variational Problems Pitman Publishing Program, Boston, pp. 1-8.

NADER, G., 2002, "Desenvolvimento de Técnicas de Caracterização de Transdutores Piezelétricos”, tese de doutorado, São Paulo - Brasil, Escola Politécnica da USP.

NAILLON, M.; Coursant, R. H. e Besnier, F., 1983, “Analysis of piezoelectric structures by a finite element method", ACTA Eletronica, Vol 25, No 4, pp. 341-362. 
NEWNHAM, R. E.; Dogan, A.; Xu, Q. C.; Onitsuka, K.; Tressler, J. e Yoshikawa, S., 1993, "Flextensional "MOONIE” Actuators", IEEE Ultrasonics Symposim, pp. 509-512.

NISHIWAKI, S., Frecker, M.I., Min, S., Kikuchi, N., 1998, “Topology Optimization of Compliant Mechanisms using the Homogenization Method", International Journal of Numerical Methods in Engineering, vol. 42, pp. 535-559.

NISHIWAKI, S., Saitou, K., Min, S., Kikuchi, N., 2000, 'Topology Design Considering Flexibility Under Periodic Loads", Struct Multidisc Optim, Springer-Verlag, 19, pp 4-16.

ODEN, J.T., Kikuchi, N., Song, Y.J., 1982, "Penalty-Finite Element Methods for the Analysis of Stokesian Flows", Computer Methods in Applied Mechanics and Engineering, vol. 31, pp. 297-329.

OLHOF, N., Bendsøe, M.P., Rasmussen, J., 1991, "On CAD-integrated Structural Topology and Design Optimization", Computer Methods in Applied Mechanics and Engineering, 89, pp 259-279.

OLHOFF, N., Kog, L., Thomsen, J., 1993, “Bi-material Topology Optimization”, In: Kerskovites J., editor, Structural Optimization, 93, COPPE/Federal University of Rio de Janeiro, RJ, Brazil, pp. 327-334.

ONITSUKA, K.; Dogan, A.; Tressler, J. F.; Xu, Q.; Yoshikawa, S. e Newnham, R. E., 1995, "Metal-Ceramic Composite Transducer, The "Moonie" ", Journal of Intelligent Material Systems and Structures, Vol 6, pp. 447-455. 
OSTERGAARD, D. F.; Pawlak, T. P., 1986, “Three-Dimensional Finite Elements for Analyzing Piezoelectric Structures”, IEEE Ultrasonics Symposim, pp. 639644.

PARK, Y. K., 1995, "Extensions of Optimal Layout Design Homogenization Method", Phd.D. Dissertation, University of Michigan.

PEDERSEN, C.B.W, Buhl, T., Sigmund, O., 2001, "Topology Synthesis of Large Displacement Compliant Mechanics”, International Journal for Numerical Methods in Engineering, 50, pp. 2683-2705.

PEREIRA, J. T., 2001, “Otimização Topológica de Componentes Mecânicos com restrições sobre o critério de falha material", tese de doutorado, FlorianópolisS.C. - Brasil, Universidade Federal de S.C..

PETERSSON, J., Sigmund, O., 1998, "Slope constrainded topology optimization", International Journal for Numerical Methods in Engineering, v. 41, pp. 14171434.

POULSEN, T. A., 2002, "A simple scheme to prevent checkerboard patterns and one-node connected hinges in topology optimization”, Structut Multidisc Optim, vol. 24, pp. 396-399.

PRAGER, W., 1974, “A Note on Discretized Michell Structures", Computer Methods in Applied Mechanics and Engineering, 3, pp. 349-355. 
PRESS, W.H., Teukolsky, S.A., Vetterling, W.T., Flannery, B.P., 1999, "Numerical Recipes in C - The Art of Scientific Computing”, pp. 430-444, Cambridge University Press.

RISTIK, V. M., 1983, "Principle of Acoustic Devices” John Wiley \& Sons, New York, USA.

RIETZ, A., 2001, "Sufficiency of Finite Exponent in SIMP (Power Law) Methods", Struct Multidisc, Springer-Verlag, 21, pp. 159-163.

ROZVANY, G.I.N., Olhoff, N., Cheng, K., Taylor, J.E., 1982, "On the Solid Plate Paradox in Structural Optimization", Journal of Structural Mechanics, 10-1, 132.

ROZVANY, G.I.N., ZHOU, M., 1991, “The COC Algorithm, Part I: Cross Section Optimization or Sizing", Computer Methods in Applied Mechanics and Engineering, 89, pp 281-308.

ROZVANY, G.I.N., Bendsøe, M. P., e Kirsch, U., 1995, "Layout Optimization of Structures”, Applied Mechanics Reviews, 48.2, pp. 41-119.

SIGMUND, O., 1996, "On the Design of Compliant Mechanisms using Topology Optimization", Mechanics of Structures and Machines, 25, pp. 495-526.

SIGMUND, O., 1998, "Systematic Design of Electrothermomechanical Actuators using Topology Optimization”. In Modelling and Simulation of Microsystems, Semiconductors, Sensors and Actuators, Santa Clara, California. MSM98, pp. 350-355. 
SIGMUND, O., Petersson, J., 1998b, "Numerical instabilities in topology optimization”. Struct. Optim., vol. 16, pp. 68-75.

SIGMUND, O., 1998c, "Systematic Design of Micro Actuators using Topology Optimization". In SPIE's 5th Ann. Int. Symp. on Smart Structures and Materials, Smart Electronics e MEMS, San Diego, California, SPIE, vol. 3328, pp. 23-31.

SIGMUND, O., 1999, "Topology Synthesis of Two Phase Compliant Actuator", Proceedings of the ASME Design Engineering Thecnical Conferences, Las Vegas, Nevada, USA, pp. 12-15.

SIGMUND, O., 2000, “A 99 Line Topology Optimization Code Written in Matlab”, Technical University of Denmark (www. topopt.dtu.dk).

SILVA, E.C.N., Nishiwaki, S, Kikuchi, N., 1999, "Design of Piezocomposite Materials and Piezoelectric Transducers Using Topology Optimization Part II", Archives of Computational Methods in Engineering, Vol 6, No 3, pp. 191-222.

SILVA, E.C.N, Nishiwaki, S., Kikuchi, N., 2000, “Topology Optimization Design of Flextensional Actuators", IEEE Transactions on Ultrasonics, Ferroelectrics and Frequency Control, 47, pp. 657-671.

SUZUKI, K.; Kikuchi, N., 1991, "A Homogenization Method for Shape and Topology Optimization", Computer Methods in Applied Mechanics and Engineering, 93, pp. 291-318. 
SVANBERG, K., 1987, "A Homogenization Method for Shape and Topology Optimization", Computer Methods in Applied Mechanics and Engineering, 93, pp. 291-318.

SWAN, C., KOSAKA, I., 1997, "Voigt-Reuss Topology Optimization for Structures with Linear Elastic Material Behaviour", International Journal of Numerical Methos in Engineering, Vol. 40, No. 1, pp. 3033-3057.

THOMSEN, J., 1992, "Topology Optimization of Structures Composed of One or Two Materials", Structural Optimization, 5, pp. 108-115.

THOMAS, H. L., Vanderplaats, G. N., Shyy, Y. K., 1992, “A Estudy of Move Limit Adjustment Strategies in the Approximation Concepts Approch to Structural Synthesis", AIAA-VMA Engineering, pp. 507-512.

TIMOSHESKO, S.P., Goodier, J.N, 1980, "Teoria da Elasticidade”, $3^{\text {a }}$ ed., Guanabara Dois.

VANDERPLAATZ, G., 1984, "Numerical Optimization Techniques for Engineering Design: with Applications". McGraw-Hill.

XU, Q. C.; Dogan, A.; Thessler, J.; Yoshikawa, S. e Newnham, R. E., 1991a, “Ceramic-Metal Composite Actuator”, IEEE Ultrasonics Symposim, pp. 923928.

XU, Q. C.; Yoshikawa, J. R.; Belsick, J. R. e Newnham, R. E., 1991b, "Piezoelectric Composites with High Sensitivity and High Capacitance for Use at High Pressures" IEEE Transactions on Ultrasonics Ferroelectrics and Frequency Control, Vol 38, No 6, pp. 634-639. 
YANG, R.J., Chuang, C.H., 1994, “Optimal Topology Design Using Linear Programming", Computer and Structures, 52-2, pp. 265-275.

YIN, L., Yang, W., 2000, “Topology Optimization for Tunnel Support in Layered Geological Structures", International Journal for Numerical Methods in Engineering, 47, pp. 1983-1996.

YOO, J., Kikuchi, N., 2000, "Topology Optimization in Magnetic Fields Using Homogenization Design Method”, International Journal for Numerical Methods in Engineering, 48, pp. 1463-1479.

ZHOU, M., Rozvany, G.I.N, 1991, “The COC Algorithm, Part II: Topological, Geometrical, and Generalized Shape Optimization”, Computer Methods in Applied Mechanics and Engineering, 89, pp 309-336. 